\title{
Acknowledgment to Reviewers of Applied Sciences in 2020
}

\author{
Applied Sciences Editorial Office
}

MDPI AG, St. Alban-Anlage 66, 4052 Basel, Switzerland

Citation: Applied Sciences Editorial Office. Acknowledgment to Reviewers of Applied Sciences in 2020. Appl. Sci. 2021, 11, 1108. https:// doi.org/10.3390/app11031108

Published: 26 January 2021

Publisher's Note: MDPI stays neutral with regard to jurisdictional claims in published maps and institutional affiliations.

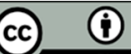

Copyright: (c) 2021 by the author. Licensee MDPI, Basel, Switzerland. This article is an open access article distributed under the terms and conditions of the Creative Commons Attribution (CC BY) license (http://creativecommons.org/licenses /by/4.0/).

Peer review is the driving force of journal development, and reviewers are gatekeepers who ensure that Applied Sciences maintains its standards for the high quality of its published papers. Thanks to the cooperation of our reviewers, in 2020, the median time to first decision was 15 days and the median time to publication was 35 days. The editors would like to express their sincere gratitude to the following reviewers for their precious time and dedication, regardless of whether the papers were finally published:

Aamir, Muhammad

Aarniovuori, Lassi

Aasa, Ulrika

Aase, Reyes

Abad, Begoña

Abah, Obinna

Abainia, Kheireddine

Abalasei, Aurelia Beatrice

Abanteriba, Sylvester

Abarca-Sos, Alberto

Abaspur Kazerouni, Iman

Abate, Giada

Abate, Lorenzo

Abatzoglou, Nicolas

Abawajy, Jemal

Abaza, Osama A.

Abbas, Farhat

Abbasi Layegh, Mahmood

Abbasi, Hamid

Abbasi, Ubaid

Abbasnia, Arash

Abbaspour, Aiyoub

Abbatangelo, Marco

Abbes, Boussad

Abbod, Maysam

Abbruzzo, Antonino

Abdala, Ahmed

Abdalla, Ahmed

Abdallah, Ali

Abdallah, Shaaban

Abdel Wahab, Omar

Abdelazim, Sameh

Abdelaziza, Sherif L.

Abdelgader, Hakim

Abdelgawwad, Ahmed
Abdeljaber, Osama

Abdelkader, Amr

Abdelkefi, Abdessattar

Abdellatef, Mohammed

Abdellatif, Mohamed

Abdelmaksoud, Ahmed

Abdelrahman, Mohamed

Abdelrazec, Ahmed H. M.

Abdelsalam, Mahmoud

Abdelsalam, Sara I.

Abdelwahed, Sameh

Abdi, Ghasem

Abdo, Ahmad

Abdo, Peter

Abdul-Aziz, Ali

Abdulhammed, Razan

Abdulkhaleq, Ahmed

abdullah, Abu Yousuf Md

Abdullah, Oday Ibraheem

Abdullah, Tariq

Abdulmajeed, Wael

Abdur, Rahim

Abed, Eyad H.

Abedi, Reza

Abeendranath, abhinav Kalathiparambil

Abegão, Luis

Abel, Markus

Abeliotis, Konstadinos

Abella, Laura Sánchez

Abeni, Luca

Abenza, Pedro Pablo Garrido

Aberšek, Boris

Abhijit, Saha

Abioye, Ayodeji Opeyemi

Abir, Muhammad 
Abisset-Chavanne, Emmanuelle

Aboagye-Sarfo, Patrick

Abolghasemi, Vahid

Abonyi, János

Abot, Jandro

Abramović, Borna

Abramovich, Haim

Ábrányi-Balogh, Péter

Abrate, Serge

Abratkiewicz, Karol

Abreu, António

Abreu, Tiago

Abrishambaf, Omid

Abrol, Vinayak

Abruzzese, Donato

Abshaev, Magomet

Abu Ghazaleh, Haitham

Abubakr, Neamat Hassan

Abu-Lebdeh, Taher

Abu-Mahfouz, Issam

Aburumman, Nemer

Abusharbeh, Mohmad T.

Abu-Zeid, Nasser

Acampa, Giovanna

Acar, Pinar

Accardo, Grazia

Accornero, Federico

Accorsi, Gianluca

Accorsi, Riccardo

Acedo, Jeella Z.

Aceituno, Javier Fernández

Aceti, Antonio

Achchaq, Fouzia

Achim, Cristina

Aciu, Claudiu

Aciu, Lia E.

Acken, John M.

Acker, Jason P.

Acomi, Nicoleta

Acquila-Natale, Emiliano

Actis Grande, Marco

Acuna, Edgar

Acuña, Luis

Adachi, Shinichiro

Adam, Deptuła

Adam, George

Adam, Glowacz

Adamcova, Renata

Adamczyk, Joanna

Adamczyk, Marcin

Adamek, Jakub
Adamik, Anna

Adamopoulos, Efstathios

Adamopoulos, Stergios

Adamowicz, Mieczysław

Adams, Jacob

Adams, James

Adams, Mike

Adamski, Mariusz Robert

Adamski, Miroslaw

Adamus, Grażyna

Adamus-Białek, Wioletta

Adarraga, Itziar

Adawy, Alaa

Addepalli, Balasubrahmanyam

Addona, Fabio

Ade, Nilesh

Adeagbo, Waheed A.

Adegboye, Mutiu Adesina

Adekunle, Timothy $\mathrm{O}$.

Adel, Amr

Adela Iuga, Cristina

Adelantado, Ferran

Adell, Teresa

Adelmann, Benedikt

Adeloju, Samuel

Adeogun, Ramoni

Adesina, Adeyemi

Adeyemi-Ejeye, Femi

Adhikari, Rajdeep

Adhikari, Ram

Adhikari, Ramesh

Adi, Wael

Adigun, Oludoyin

Adjaoud, Omar

Adkins, Kevin A.

Adl, Sina

Adolphs, Julian

Adorjan, Michael

Adorno, William

Adrover, Alessandra

Aelterman, Jan

Aerts, Sam

Afa Michael, Inikuro

Afanasev, Andrei

Afenyo, Mawuli

Affatato, Saverio

Afghan, Syeda Adila

Afify, Ahmed

Afonso, Clélia Neves

Afrashtehfar, Kelvin

Afroz, Zakia 
Afsari, Morteza

Afshani, Alireza

Afsharipour, Elnaz

Afzaal, Hassan

Agana, Norbert

Agarwal, Girish

Agarwal, Rachit

Agarwala, Ranjeet

Agathoklis, Pan

Agaton, Casper Boongaling

Agee, Philip

Aggogeri, Francesco

Aghajani, Haleh

Aghakhani, Amirreza

Aghazadeh, Fereydoun

Agili, Sedig S.

Agiwal, Mamta

Agnusdei, Giulio Paolo

Agonafir, Mesfin Belayneh

Agre, Gennady

Agrela, Francisco

Agresti, Giorgia

Agriopoulou, Sofia

Aguasca-Colomo, Ricardo

Agüero, Ramon

Aguilar, Miguel Pic

Aguilera, Pedro A.

Aguiló-Aguayo, Noemí

Aguinaga, Iker

Aguirresarobe, Robert $\mathrm{H}$.

Agustin, Dominique

Aharon, David Yechiam

Ahishali, Mete

Ahlfeld, Tilman

Ahlström, Peter

Ahluwalia, Pankaj

Ahma, Luan

Ahmad, Fahim

Ahmad, Hafiz Waqar

Ahmad, Jawad

Ahmad, Muhammad

Ahmad, Tanveer

Ahmad, Wasim

Ahmadi, Seyed Farzad

Ahmadi, Soha

Ahmadivand, Arash

Ahmed, Asif

Ahmed, Hafiz

Ahmed, Ishtiaq

Ahmed, Mohiuddin

Ahmed, Mukhtar
Ahmed, Rafiuddin

Ahmed, Sheikh

Ahmed, Sheraz

Ahn, Chang Wook

Ahn, Hyung Keun

Ahn, Jin-Soo

Ahn, Jooeun

Ahn, Kwangwon

Ahn, Kyoung

Ahn, Sangdoo

Ahn, Seokyoung

Ahn, Yong

Ahrens, Richard A.

Ahsan, Md Mominul

Ahsan, Md. Shamim

Ahsani, Vesal

Ahuir-Torres, Juan

Aichinger, Philipp

Aiello, Domenico

Aiello, Francesca

Aiello, Orazio

Aikawa, Akira

Aikins, Deane

Aimo, Alberto

Aissa, Brahim

Aissa, Mohamed Hassanine

Aivazidou, Eirini

Ajejas, Fernando

Akafuah, Nelson K.

Akama, Makoto

Akan, Cigdem

Akasaka, Shuichi

Akbar, Sheikh

Akbarinia, Arash

Aki, Tsunehiro

Akıncilar, Semih Can

Akinsolu, Mobayode O.

Akira, Ishibashi

Akira, Matsumoto

Akitsu, Tetsuya

Akiyama, Iwaki

Akkaya, Ilge

Akkermans, Rinie

Akpan, Emmanuel I.

Akrami, Mohammad

Aksac, Alper

Aksanli, Baris

Aksenenko, Eugene V.

Aktan, Ahmet Emin

Aküzüm, Bilen

Al Hammoshi, Mayyada 
Al Sabouni-Zawadzka, Anna

Al Souki, Karim

Al-Abadi, Ali

Al-Aboosi, Fadhil

Alabrudziński, Sławomir

Alaerts, Luc

Alaimo, Alessandro

AlAli, Abdulrahman

Alam, Arif Ul

Alam, Marzia

Alam, Miah Md Ashraful

Alam, Mohammad Parvez

Alam, Mollah Rezaul

Alam, Shaurav

Alameddin, Shadi

Al-Ameri, Talib

Alamiri, Nasser

Al-Amri, Mohammad

Alander, Jarmo

Al-Anssari, Sarmad

Al-antari, Mugahed A.

Alapati, Satish B.

Alappattu, Denny P.

Alarabi, Louai

Alasali, Feras

Al-Asmar, Asmaa

Alava, Juan Jose

Alavi, Saman

Alazab, Mamun

Albano, Gianluigi

Albarracin, Ricardo

Albatici, Rossano

Albendín-García, Luis

Alberghini, Matteo

Alberini, Federico

Albert, Justin

Alberta, Lucchese

Alberti, Luca

Alberti, Luigi

Alberti, Tommaso

Alberto Villegas, Julian

Albetran, Hani

Albiez, Matthias

Alborz, Niknam

Alboul, Lyuba

Albu, Adriana

Albuquerque, M. T. D.

Alcalá Herrera, Rafael

Alcaraz, Cristina

Alcaraz, Lorena

Alcaraz-Carrillo de Albornoz, Vicente
Alcarria, Ramón

Alcayde, Alfredo

Alcázar-Ortega, Manuel

Alcolea, Veronica

Al-Dujaili, Emad

Aldwell, Barry

Aleixandre, Manuel

Aleksandrova, Mariya

Alekseenko, Alexey

Aleksey, Nikulov

Aleksieva, Lyubka

Alemayehu, Fisseha M.

Aleshkina, Svetlana S.

Alessandra, Saponieri

Alessandri, Angelo

Alessandro, Torchio

Alessi, Antonino

Alessia, Allevi

Aletta, Francesco

Alexakis, Dimitrios E.

Alexakos, Christos

Alexan, Wassim

Alexandridis, Georgios

Alexandru, Burde

Alexandru, Cătălin

Alexiev, Ulrike

Alexopoulos, Nikolaos

Alexovič, Michal

Alfei, Silvana

Alfeo, Antonio Luca

Alferez, Fernando

Alfian, Ganjar

Alfonso, Gago-Calderón

Alfranca, Oscar

Algar, Juan

Algergawy, Alsayed

Alghamdi, Ibrahim

Algieri, Angelo

Alguacil De La Blanca, Gerardo

Al-Gumaei, Yousef

Al-Hadhrami, Tawfik

Alhamdani, Wasim

Alhasheem, Mohammed

Al-Hussein, Abdullah

Ali Abbas Kazmi, Syed

Ali Ansari, Sajid

Ali, Aamer

Ali, Abdul (Italy)

Ali, Abdul (UK)

Ali, Farman

Ali, G. G. Md. Nawaz 
Ali, Haider (Norway)

Ali, Haider (USA)

Ali, Howbeer Muhamad

Ali, Jehad

Ali, Majid

Ali, Mohamed M.

Ali, Naser

Ali, Nazakat

Ali, Rashid

Ali, Usman

Aliabadi, Amir A.

Aliabadi, M. H.

Aliannejadi, Mohammad

Aliaño-González, Maria

Al-Ibadi, Alaa

Alibakhshikenari,

Alin, Bosioc

Alinejad-Rokny, Hamid

Alipour, M. H.

Alisaac, Elias

Alissa, Samieh

Aliyu, Musa

Alizadeh, Babak

Alizadeh, Morteza

Alizadeh, Vahid

Alizadehsalehi, Sepehr

Al-Jawad, Jafar Y.

Al-Juboori, Muqdad

Alkaff, Saqaff Ahmed Abdulrahman

Alkan, Bugra

Alkasrawi, Malek

Alkhalaf, Haitham

Al-Khalidi, Abdullah

Alkhalifeh, Khaldoun

Al-Kheetan, Mazen

Allegra, Eugenia

Allegro, Gianluca

Allen, Thomas

Allendes, Rodolfo

Allouche, David

Al-Madhhachi, Abdul-Sahib

Almadhor, Ahmad

Almandoz, Gaizka

Almansa, Francisco

Almasian, Mitra

Almasri, Maher

Al-Matarneh Sattam,

Almeida Ferreira, Hugo

Almeida Júnior,

Almeida, Arminda

Almeida, Bernardo Gonçalves
Almeida, Fernando

Almeida, João

Almeida, Joao Emilio

Almeida, Luis Eduardo

Almeida, Manuela

Almeida, Marisa

Almeida, Miguel

Almenárez, Florina

Almendros-Jiménez,

Almodovar-Melendo, Jose-Manuel

Almuneau, Guilhem

Al-Muqdadi, Sameh W.

Al-Musaed, Amjad

AL-Naji, Ali

Al-Najjar, Basim

Al-Nasrawi, Ali K. M.

Alobaid, Falah

Alodjants, Alexander

Aloisio, Angelo

Alomari, Abdullah

Alonso, Miguel A.

Alonso, Rafael

Alonso-García, María

Aloqaily, Moayad

Alotta, Gioacchino

Al-Qaderi, Mohammad

Al-Qadi, Imad L.

Al-Radhi, Mohammed Salah

Al-Rimawi, Fuad

Al-Sabah, Salam

Alsadik, Bashar

Alsajri, Firas Ahmed

Al-Samarraie, Hosam

Al-Samman, Ahmed M.

Al-Saqaf, Walid

Alshammari, Abdullah

Alshazly, Hammam

Al-Shibaany, Zeyad

Alsina-Pagès, Rosa Ma

Alsinglawi, Belal

Alsop, Roger

Alt Murphy, Margit

Altaf, Amir

Altaie, Ala

Altaisky, Mikhail

Altammar, Hussain

Altan, M. Cengiz

Altavilla, Stefania

Altay, Okyay

Altin, Müfit

Altosole, Marco 
Aluigi, Luca

Álvarez Ariza, Jonathan

Alvarez Morales, Lidia

Álvarez Pardiñas, Ángel

Alvarez Sanchez, David

Alvarez, José

Álvarez, Paloma

Alvarez, Rafael

Alvarez-Builla, Julio

Alvarez-Serrano, Inmaculada

Alvarez-Suarez, Jose

Álvarez-Tey, Germán

Álvarez-Tuñón, Olaya

Álvarez-Vázquez, Miguel Angel

Alvero Cruz, José Ramón

Alves De Sousa, Ricardo J.

Alves Durães, Dalila

Alves, Eliana

Alves, Elsa

Alves, Joel

Alves, Luis

Alves, Luis Nero

Alves, Ricardo

Alves, Thais Da C. L.

Alwaeli, Mohamed

Alwattar, Tahseen A.

Aly, Karim

Al-Yaari, Amen

Alzoubi, Mahmoud A.

AlZubaidi, Abbas K.

Amado, Miguel

Amaechi, Chiemela Victor

Amalevičiūtè Volungè, Kristina

Amaly, Noha

Amanatiadis, Stamatios

Amano, Kinjiro

Amanowicz, Łukasz

Amaricai, Elena

Amaro, José Pedro

Amaro-Mellado, José L.

Amarowicz, Ryszard

Amat, Esteve

Amato, Umberto

Amatya, Reja

Ambrosetti, Alberto

Ambrosino, Fabrizio

Ambs, Pierre

Ambu, Rita

Amedeo, Amoresano

Ameduri, Salvatore

Amelard, Robert
Amer, Moh (USA)

Amer, Mohammed (Taiwan)

Amer, Yousef

Amezcua, Javier

Amici, Julia

Amilusik, Mikolaj

Amin, Farhan

Amin, Muhammad Junaid

Aminbaghai, Mehdi

Amine, Aziz

Amineh, Reza K.

Amirat, Yassine

Amiri, Sam

Amirov, Abdulkarim

Amisano, Fabrizio

Amitonova, Liubov

Ämmälä, Ari

Ammirabile, Angela

Amnache, Amrid

Amo, Daniel

Amoako, Kagya

Amoozgar, Zohreh

Amores Arrocha, Antonio

Amorim, Marlene

Ampatzidis, Dimitrios

Ampeliotis, Dimitris

Amundarain Irizar, Aiert

Amza, Catalin

An, Chunjiang

An, Dongbo

An, Heungjo

An, Hyosung

An, Ran

Ana Belén

Anagnostis, Athanasios

Anagnostopoulos, Costas A.

Anagnostopoulos, Marios

Anagnostopoulou, Afroditi

Anagnostou, Dimitris

Anajafi, Hamidreza

Anan, Go

Anand, Prashant

Anand, Vijay

Anani, Nader

Ananthakrishnan, Soundaram Jeevarathinam

Ananthan, Sowmya

Anastasiou, Antonios

Anastasiou, Athanasios

Anatolievich Zakharenko, Aleksey

Anca, Filimon

Anceschi, Anastasia 
Anco, Daniel J.

Ancora, Daniele

Andaluri, Gangadhar

Anderlini, Letizia

Andersen, Brian Møller

Andersen, Jens Christian

Anderson, Travis M.

Andersone, Ilze

Andert, Jakob

Ando, Hidetoshi

Ando, Keita

Ando, Masaki

Andoga, Rudolf

Andrade Pais, Luis José

Andrade, Eugénia De

Andrade, José Pedro

Andrades-Peña, Francisco Javier

Andreasen, Rasmus

Andreaus, Ugo

Andreea, Hegyi

Andreev, Konstantin

Andreev, Vladimir V.

Andreeva, Marina

Andrei, Gabriel

Andrei, Jean-Vasile

Andrei, Laurentia

Andreoni, Giuseppe

Andres, Juan

Andrew, Keith

Andrews, Aaron Maxwell

Andrews, David L.

Andria, Gregorio

Andric, Jelena

Andrieu, Guillaume

Andrieux, Guillaume

Andrievsky, Boris

Andriolli, Nicola

Andriollo, Tito

Andrisani, Andrea

Andročec, Darko

ANDROJNA, Andrej

Andronikos, Theodore

Andros Dubraja, Lidija

Androutsos, Nikolaos A.

Andryushin, Konstantin P.

Andrzej Kolator, Bronisław

Andrzej, Michalski

Andrzej, Rusin

Andwari, Amin Mahmoudzadeh

Anesi, Alexandre

Aneziris, Olga
Ang, Chee Wei

Ang, Linus Yinn Leng

Ang, Yee Sin

Angeli, Elena

Angelici, Robert J.

Angélico Gonçalves, Maria José

Angelidis, Pantelis

Angelino, Donato

Angelopoulou, Matina V.

Angelov, Borislav

Angelov, George

Angelova, Mihaela

Anggraini, Sri Ayu

Anggraini, Vivi

Anghel, Larisa

Anghelescu, Petre

Angizi, Shayan

Angrecka, Sabina

Anguera, Jaume

Ania-Castañon, Juan Diego

Anifandis, George

Anim-Danso, Emmanuel

Anis, Badawi

Anita Ioana, Visan

Anitescu, Cosmin

Anjos, Ofélia

Anna, Loboda

Annala, Leevi

Annalisa, Natali Murri

Annamdas, Venu Gopal Madhav

Annunziata, Giuseppe

Añorga Benito, Javier

Anouar, Alami

Ansaf, Bahaa

Ansari, Kutubuddin

Ansell, Troy Y.

Anselm, Blumer

Ansermet, Jean-Philippe

Anthony, Carl

Anthony, Mark

Anthony, Stephen

Antipov, Oleg L.

Antlej, Kaja

Antoine, Claire

Antonela Antoniu, Sabina

Antonelli, Laura

Antoni, Jerome

Antoniadou, Maria

Antoniazzi, Francesco

Antonino, Famulari

António Correia, José 
Antonio, Formisano

Antonkiewicz, Jacek

Antonopoulos, Angelos

Antonopoulos, Christos S.

Antonopoulou, Georgia

Antonucci, Francesca

Antoszewski, Bogdan

Antov, Petar

Anttu, Nicklas

Antunes Do Carmo, José Simão

Antunes, Carlos M. Correia

Antunes, Joana

Antunes, Marcelo

Antunes, Mário

Antunes, Rodrigo

Anuganti, Murali

Anugwom, Ikenna

Anukam, Anthony

Anupam, Kumar

Anwar, Mohammad

Anwar, Suzan

Anwar, Syed Muhammad

Anwary, Arif Reza

Anweiler, Stanisław

Anysz, Hubert

Anyżewska, Anna

Anzano, Jesus M.

Aoki, Mutsumi

Aono, Hikaru

Aparicio-Blanco, Juan

Aparicio-Pardo, Ramon

Apiecionek, Łukasz

Apinyan, Vardan

Aple, Derek B.

Apollo, Michal

Apollonio, Ciro

Apostol, Marian

Apostoluk, Aleksandra

Appel, Arthur G.

Appelbaum, Joseph

Appell, Michael

Appetecchi, Giovanni Battista

Aprodu, Iuliana

Aqlan, Faisal

Arabi, Saeed

Arabo, Abdullahi

Aradilla, David

Aragoneses, Andrés

Aramendia, Iñigo

Aramrattana, Maytheewat

Aranaz, Paula
Aranda-Escolástico, Ernesto

Aranda-Ruiz, Josue

Arandjelović, Ognjen

Araniti, Fabrizio

Arantzazu Gomez, María

Arato, Elisabetta

Araujo, Alvaro

Araújo, Noelia

Araújo, Ricardo Jorge

Aravind, Padmanabhan K.

Arbabi, Hadi

Arbanas, Željko

Arbeiter, Florian

Arceo, Juan Carlos

Arcia-Moret, Andrés

Arcos González, Pedro

Arcos, Robert

Arcudi, Francesca

Arcuri, Natale

Arczewska, Marta

Ardelean, Lavinia Cosmina

Ardigò, Luca Paolo

Ardissono, Liliana

Arefnezhad, Sadegh

Arena, Andrea

Arena, Fabio

Arena, Maurizio

Arena, Simone

Arenas, Eva

Arendas, Peter

Aresti, Lazaros

Argente, María-José

Argenziano, Pasquale

Arghir, Mihaï

Argilaga, Albert

Argyroudis, Sotiris

Ariansyah, Dedy

Arias Trujillo, Juana

Arias-Alvarez, María

Aricò, Fabio

Aricò, Pietro

Arienzo, Michele

Arif, Ali Talib

Arima, Takuji

Ariño, Agustín

Arion, Cristian

Arion, Felix

Aristia, Gabriela

Ariyani, Winda

Ariyur, Kartik B.

Arjona, Rosario 
Arjoune, Youness

Arjunan, Arun

Arkadiusz, Dziedzic

Arkas, Michael

Arkusz, Katarzyna

Armada Da Silva, Paulo

Armada, Manuel A.

Armaroli, Andrea

Armengol, Ramón

Armin, Zare

Armstrong, Paul

Armstrong, Stuart

Arnaiz, Ana

Arnal, Josep

Arnau, Carles B.

Arnaubec, Aurélien

Arnaud, Recoquillay

Arnbjörnsson, Einar

Arndt, Daniel

Arnold, Philipp

Årøen, Asbjørn

Arooj, Fatima

Arora, Shashank

Arosio, Paolo

Arrabito, Giuseppe

Arráez-Aybar,

Arraiano, Irene

Arranz, Beatriz

Arranz-Justel, Jose Juan

Arrebola, Manuel

Arregui, Maria

Arribas, Juan Ignacio

Arrighetti, Andrea

Arrigo, Rossella

Arrigoni, Roberto

Arroyo Cardoso, Filipe

Arroyo Ohori, Ken

Arshad, Adeel

Arshadi, Amir

Arsiccio, Andrea

Artacho, Bruno

Artale, Giovanni

Artal-Sevil, Jesús Sergio

Arteaga, Jesús Fernández

Arteconi, Alessia

Artemov, Mikhail

Artesani, Alessia

Artese, Giuseppe

Artese, Maria Teresa

Arteta, Alberto

Artetxe, Beñat
Arthur, Chris

Artiemjew, Piotr

Artola, Garikoitz

Artur, Mariana Aline Silva

Aruga, Kazuhiro

Arvanitis, Konstantinos

Aryal, Yog

Aryan, Hadi

Arzamendi, Gurutze

Asa'ad, Farah

Asadi, Amin

Asadi, Behzad

Asadi, Ehsan

Asadi, Houshyar

Asadzadeh, Mohammad Zhian

Asakura, Takumi

Asami, Toshihiko

Asayama, Shoichiro

Ascencio-Vásquez, Julián

Aschonitis, Vassilis

Ascione, Giacomo

Aseev, Vladimir Anatolevich

Asensio, Gabriel

Asfin, Ruslan E.

Asgari, Somayyeh

Ashbrook, Aaron R.

Ashfaq Khan, Muhammad

Ashkenazi, Dana

Ashraf, Fahmidah U.

Ashraf, Imran

Ashton, Triss

Asif, Muhammad Bilal

Asif, Rameez

Asim, Umair Bin

Asimina, Stamatelopoulou

Asín Prieto, Guillermo

Asins-Velis, Sabina

Askarinejad, Sina

Askes, Sven

Aslani, Mohamad

Aslansefat, Koorosh

Ašmontas, Steponas

Asnafi, Nader

Aspatwar, Ashok

Asquith, Christopher R. M.

Assad, Mamdouh

Assadian, Francis

Assadi-Langroudi, Arya

Assaf, Tareq

Assante, Dario

Assari, Shervin 
Assefi, Mohammad

Assenza, Giovanni

Assi, Lateef N.

Assirelli, Alberto

Assirelli, Elisa

Assmann, Marc

Assunção, Pedro

Astakhov, Viktor P.

Astakhova, Kira

Asteris, Panagiotis G.

Astolfi, Arianna

Astudillo León,

Asvapathanagul, Pitiporn

Aszodi, Attila

Atanassova, Stefka

Atencia, Miguel

Ates, Cihan

Athanasiadis, Vassilis

Athanasiou, Apostolos

Atif, Rasheed

Atkins, Ella M.

Atkinson, Alan D.

Atkinson, Anthony C

Atkinson, Henry Dushan E.

Atkison, Travis

Atroshchenko, Elena

Attanasio, Chiara

Attia, Nour F.

Attia, Sameh

Attila, Gere

Atuchin, Victor

Atzori, Giulia

Aubry, Pascal

Audrito, Giorgio

Auersch, Lutz

Aughey, Robert

Augustine, Robin

Augusto, Hugo

Augustynek, Martin

Augustyniak, Adrian

Aung, Kendrick

Aurelian, Isar

Aureliano, Manuel

Ausiello, Pietro

Ausloos, Marcel

Avadanei, Mihaela

Avanaki, Kamran

Avanaki, Mohammad R. N.

Avdicevic, Maja Zebic

Aversa, Alberta

Avgerinou, Maria. D
Avgeropoulos, Apostolos

Ávila-Gutiérrez, Maria Jesús

Aviles, Francesc Xavier

Avilés, Maria Dolores

Avino, Pasquale

Avitabile, Gianfranco

Avram, Camelia

Avramidis, Pavlos

Aw, Kean

Awin, Faroq

Awni, Rasha A.

Awolusi, Ibukun Gabriel

Awrejcewicz, Jan

Awtoniuk, Michał

Axenopoulos, Apostolos

Axiotis, Evangelos

Ayabe, Yoshiko

Ayad, Amira

Ayadi, Abderrahmane

Ayala, Julia

Ayar, Pooyan

Aycan, Murat

Aydin, Serdar

Aydin, Ümit

Ayed, Samah Ben

Ayesta, Igor

Aykas, Didem

Ayre, David

Aysu, Aydin

Ayub, Ahmad Syahrir

Azab, Mohamed

Azadfar, Mohammadali

Azam, Sami

Azarova, Olga A.

Azimi, Mohsen

Aziz, Abdul Rashid

Aziz, Fayeem

Aziz, Muhammad

Aziz, Mustafa

Azizi, Vahid

Azman, Samet

Azmat, Muhammad

Azmi, Azwan Iskandar

Azorín-López, Jorge

Azzini, Antonia

B N, Pavan Kumar

B. Carda Castelló, Juan

Babaei, Alireza

Babak, Maria V.

Babbar, Rohit

Babic, Darko 


\begin{tabular}{|c|c|}
\hline Babič, František & Baghdadi, Ali \\
\hline Babic, Vesna & Baghdadi, Amir \\
\hline Babichev, Sergii & Baghdadi, Nicolas \\
\hline Babicz, Marek & Baghdadi, Ziad D. \\
\hline Babilio, Enrico & Bagheri, Zahra \\
\hline Babojelić, Martina & Baghi, Quentin \\
\hline Baboly, Mohammadhosein Ghasemi & Bagiu, Iulia-Cristina \\
\hline Babu, Baniya & Baglieri, Andrea \\
\hline Babusiak, Branko & Baglieri, Jacopo \\
\hline Baca, Jose & Baglioni, Michele \\
\hline Bacalum, Mihaela & Bagnerini, Patrizia \\
\hline Bacanin, Nebojsa & Bagnoli, Franco \\
\hline Bacchiocchi, Simone & Bagüés, Ana \\
\hline Bacci, Christian & Bagui, Sikha \\
\hline Bacci, David & Bahador, Bagheri \\
\hline Bacci, Stefano & Bahi, Slim \\
\hline Bacco, Davide & Bahja, Mohammed \\
\hline Bace, Edward & Bahk, Je-Hyeong \\
\hline Bachler, Martin & Bahmanpouri, Farhad \\
\hline Bačić, Boris & Bahnsen, Chris H. \\
\hline Bačić, Mario & Bahrami, Amin \\
\hline Backiel-Brzozowska, Beata & Bai, Chen \\
\hline Bączek, Katarzyna & Bai, Hua \\
\hline Bączkowicz, Dawid & Bai, Lei \\
\hline Baczynski, Dariusz & Bai, Lu \\
\hline Badagliacca, Giuseppe & Bai, Renren \\
\hline Badau, Adela & Bai, Shaoping \\
\hline Badau, Dana & Bai, Yuchao \\
\hline Badea, Gheorghe & Baiardi, Liala \\
\hline Badea, Mihaela & Baig, Zubair \\
\hline Badino, Paola & Bailey, Joshua P. \\
\hline Badretdinov, Ildar & Bailey, Michael \\
\hline Badulescu, Daniel & Bailey, Sean \\
\hline Badura, Pawel & Bailey-Davis, Lisa \\
\hline Bae, Hae-Rahn & Baimel, Dmitry \\
\hline Bae, Suk Joo & Bajan, Sarah \\
\hline Bae, Won & Bajare, Diana \\
\hline Bae, Youngchul & Bajda, Miroslaw \\
\hline Bae, Youngju & Bajec, Patricija \\
\hline Baek, Jieun & Bak, Kathrine Holmgaard \\
\hline Baek, Jong-Suep & Bak, Miklós \\
\hline Baek, Joong-Hwan & Bakalár, Tomáš \\
\hline Baek, Kwang Ryul & Bakalis, Evangelos \\
\hline Baek, Seung Ho & Bakarčić, Danko \\
\hline Baek, Soo-Whang & Bakdi, Azzeddine \\
\hline Baek, Youngbin & Bakeas, Evangelos \\
\hline Baele, Jean-Marc & Baker, Jonathon L. \\
\hline Baena, Francisco Manuel & Baker, Martin \\
\hline Bagassi, Sara & Baker, Matthew B. \\
\hline Bagdziunas, Gintautas & Bakhshande, Fateme \\
\hline Baghaie, Ahmadreza & Bakhshinejad, Ali \\
\hline
\end{tabular}


Bakhtiari, Reyhaneh

Baki, Abul B. M.

Bakka, Haakon

Bakon, Tomasz

Bakos, Levente

Bąkowski, Jarosław

Bakr, Mahmoud

Bakunowicz, Jerzy

Bała, Piotr

Balaban, Amanda E.

Balabanov, Vladimir

Bałachowski, Lech

Balamurali, Mehala

Balan, Oana

Balan, Titus

Balandin, Dmitry Vladimirovich

Balar, Nrup

Balashov, Nikolay

Balashov, Victor N.

Balasingam, Balakumar

Balasubramani, Ravindran

Balasubramanian, Karthik

Balasubramanian, Padmanabhan

Balasubramanian, Venkatraman

Balawejder, Maciej

Baláž, Peter

Balázs, Bánhelyi

Balázs, Németh

Balázs, Péter

Balbekin, Nikolay

Balcerek, Maria

Baldán Lozano, Francisco Javier

Baldassari, Sara

Baldassarre, Antonio

Baldauf, Michael

Băldean, Doru-Laurean

Baldi, Simone

Baldini, Gianmarco

Baldinozzi, Gianguido

Baldissera, Paolo

Baldisserotto, Anna

Baldoni, Andrea

Bałdowska-Witos, Patrycja

Baldry, Mark

Balducci, Fabrizio

Balduzzi, Giuseppe

Baldwin, Susan

Balena, Pasquale

Balestrieri, Eulalia

Baleta, Jakov

Balhaddad, Abdulrahman A.
Balicki, Jerzy

Baliello, Andrea

Balin, Alicja

Balint, Cornel

Bálint, Erika

Bališ, Peter

Ballard, Allison J.

Ballarotto, Giulia

Ballato, John

Balle, Frank

Ballestrin, Jesus

Balling, Ole

Ballini, Andrea

Ballo, Andrea

Balmakhtar, Marouane

Balobanov, Viacheslav

Balog, Michal

Balogh, Andrea

Balogh, Zsolt

Balsa-Barreiro, Jose

Balsi, Marco

Baltac, Vasile

Baltakys, Kęstutis

Baltazar, Luís Gonçalo Correia

Balyk, Olexandr

Balzanella, Antonio

Balzaretti, Claudia Maria

Balzer, Jan

Bamatraf, Saeed M.

Bambach, Mike

Bamiedakis, Nikos

Ban, Hyowon

Ban, Marko

Banach, Artur

Banach, Marcin

Banaitienè, Nerija

Banaś, Anna

Banaszak, Szymon

Banaszak, Zbigniew

Banaszkiewicz, Tomasz

Banchelli, Martina

Bandera, Antonio

Bandinelli, Romeo

Bandyopadhyay, Debasish

Bandyopadhyay, Supriyo

Banerjee, Abhinandan

Banerjee, Aditi

Banerjee, Ashis

Banerjee, Dishary

Banerjee, Ruman

Baneschi, Ilaria 
Bani Younes, Ahmad

Bani Younes, Maram

Bania, Piotr

Banias, Ovidiu

Baniasadi, Mahsa

Baniata, Hamza

Bank, Carl-Georg

Bańkosz, Ziemowit

Bańkowski, Wojciech

Banks, Lori

Banks, Vanessa J.

Bano, Maksim

Bañón, Fermín

Bañón, Luis

Baños Navarro, Raúl

Banožić, Marija

Bansal, Kuldeep K.

Bansmann, Joachim

Banti, Christina N.

Bantis, Filippos

Banu, Ioan-Viorel

Bao, Jie

Bao, Xiue

Bao, Yi

Baptista, Ricardo

Barabás, István

Barabas, Sorin

Barabino, Benedetto

Baragaño, Diego

Barajas, Karla Caballero

Barakabitze, Alcardo Alex

Barakh Ali, Sogra Fathima

Baraldi, Pietro

Barandiaran, Irati

Baraniecka, Anna

Baranov, Maksim V.

Baranova, Ekaterina

Baranovskii, Evgenii S.

Baranowska, Hanna

Baranski, Mariusz

Bárány, Tamás

Baranyai, László

Barati, Masoud

Barati, Ziba

Barazanchi, Abdullah

Barb, Adrian S.

Barbalinardo, Marianna

Barbancho, Ana

Barbarelli, Silvio

Barbato, Felicia Carla Tiziana

Barbera, Salvatore
Barberis, Fabrizio

Barbero, Monica

Barberopoulou, Aggeliki

Barbi, Silvia

Barbiellini, Bernardo

Barbieri, Giuseppe

Barbieri, Loris

Barbillon, Grégory

Barbir, Frano

Barbucha, Dariusz

Barbuto, Mirko

Barca, Emanuele

Barcellona, Simone

Barczak, Kamil

Bard, Delphine

Bardella, Paolo

Bardi, Giuseppe

Bardova, Kristina

Barel, Itay

Barettin, Daniele

Barez, Fred

Barfuss, Wolfram

Bargallo, Ramon

Barić, Tomislav

Barile, Claudia

Barille, Regis

Barinova, Sophia

Barisic, Ivana

Bariviera, Aurelio

Barkalov, Alexander

Barletta, Vita Santa

Barmparis, Georgios D.

Barmpounakis,

Barmpoutis, Panagiotis

Barna Iantovics, Laszlo

Barnabas, Janet

Barnes, Frank S.

Barnett, Megan

Barneva, Reneta

Barolli, Leonard

Barona, Lorena

Barone, Ettore

Barone, Pier Matteo

Baroni, Luciana

Baros, Dimitrios K.

Baroth, Julien

Barragán Martín,

Barral, Valentín

Barreca, Davide

Barreca, Francesco

Barreca, Salvatore 
Barreiro García, Joaquín

Barrell, Graham K.

Barrena-Martinez, Jesus

Barrère Cambrún, Martín

Barresi, Giacinto

Barreto Santos, Miguel

Barretta, Raffaele

Barrientos Velazquez, Ana L.

Barrientos, Antonio

Barriere, Thierry

Barrile, Vincenzo

Barroca, Bruno

Barros, Julio M.

Barroso, Álvaro Carrera

Barroso-Solares, Suset

Barrott, Jared

Barry, Carol

Barsan, Narcis

Barsi, Arpad

Barski, Marek

Barsukova, Marina

Barszczewska-Rybarek, Izabela

Bart, Austin Cory

Barthelemy, Johan

Barthelmie, Rebecca J.

Bartkowiak, Tomasz

Bartlet, Paul A.

Bartmański, Michał

Bartocci, Pietro

Bartolac, Marko

Bartold, Maciej

Bartoletti, Cipriano

Bartoli, Mattia

Bartolic, Juraj

Bartolini, Susanna

Barton, Killian

Bartoněk, Dalibor

Bartyś, Michał

Bartzas, Georgios

Bartzis, John

Barua, Shaibal

Baruffa, Giuseppe

Barukčić, Marinko

Baruque Zanon, Bruno

Baruth, Andrew

Baryannis, George

Baryshnikov, Gleb V.

Barz, Cristian

Barzee, Tyler J.

Basan, Elena

Basarab, Mikhail
Basaran, Cemal

Bascompta, Marc

Báscones, Daniel

Baselt, Ivo

Bashiri, Fereshteh S.

Basiak, Ewelina

Basic, Kresimir

Bašić, Tomislav

Basile, Valerio

Basile, Vito

Basiricò, Tiziana

Bassani, Andrea

Bassani, Marco

Bassler, Michael

Bäßler, Ralph

Bassoli, Elisa

Bastakoti, Bishnu Prasad

Bastas, Ali

Basterrechea Chertudi, Daniel

Basti, Alessio

Bastos-Arrieta, Julio

Bastrzyk, Anna

Basu, Anirban

Basu, Debashis

Basu, Saikat

Baszynski, Marcin

Batalla, Jordi Mongay

Batchuluun, Ganbayar

Bathrellos, George

Batin, Gabriel

Batista, Arnaldo

Batista, Milan

Batorek Lukač, Nina

Batrakov, Andrey

Battacone, Gianni

Battafarano, Giulia

Battaglia, Rosalia

Battah, Basem

Battarra, Mattia

Battegazzore, Daniele

Batteux, Michel

Battineni, Gopi

Battistel, Postdoc Alberto

Battisti, Fabrizio

Battistini, Simone

Battocchio, Chiara

Bauer, Brent A.

Bauer, Eike

Bauer, Elvira M.

Bauer, Jan

Bauer, Klaus 
Bauer, Maris

Bauer, Sebastian

Baumer, Stefan

Baumgarten, Martin

Baumstark-Khan, Christa

Bausch, Nils

Bause, Katharina

Bauza-Kaszewska, Justyna

Bavaresco, Luigi

Bavasso, Irene

Bawaj, Mateusz

Bayarri, Miguel Souto

Bayat, Mahmoud

Bayat, Nozhan

Bayati, Navid

Baycik, Nail

Bayomy, Ayman M.

Bayón, Alicia

Bayu Aji, Leonardus Bimo

Bayuk, Irina

Bazanowski, Maciej

Bazazian, Dena

Bazile, Didier

Bažok, Renata

Bazylko, Agnieszka

Bazzan, Marco

Beach, Thomas H.

Beane, Gary

Beard, James D.

Beata, Zima

Beatus, Tsevi

Beben, Damian

Bębenek, Ewa

Beber, Vinicius Carrillo

Bec, Krzysztof B.

Beccali, Marco

Becek, Kazimierz

Becerra, Javier

Bechtsis, Dimitrios

Beck, Brian M.

Beck, John

Beck, Nils

Beck, Yuval

Becker, Kathrin

Becker, Maik

Becker, Stefan

Becker, Till

Becker, Wilhelm

Beckham, George

Beckman, Sara

Beckmann, Volker
Bécsi, Tamás

Bede, Natalija

Bediz, Bekir

Będkowski, Bartłomiej

Bednar, Slavomir

Bednarz, Edward

Bednarz, Lukasz

Bedon, Chiara

Bedon, Frank

Będowska-Sójka, Barbara

Beenakker, Jan-Willem

Beer, Martin

Beex, Lars

Begaud, Xavier

Begg, David

Begho, Toritseju

Begines Ruiz, Belén

Begnoni, Giacomo

Behar, Alberto

Behnam, Behrouz

Behnood, Ali

Behravesh, Seyed Behzad

Behrendt, Cezary

Behrendt, Matthias

Beidaghy Dizaji, Hossein

Beilby, Mary

Beinat, Alberto

Beirão, Pedro

Beiu, Roxana-Mariana

Bejger, Artur

Beju, Livia Dana

Bekheet, Maged F.

Bekiaris, Georgios

Bel'skaya, Lyudmila

Belaïd, Abdel

Belardini, Alessandro

Belay, Birhanu

Belcher, Christina

Belcic, Ivan

Bele, Eral

Beleiu, Horia Gheorghe

Belenguer, Tomás

Belfiore, Oscar Rosario

Belgiorno, Giacomo

Belhaj, Adil

Belhallouche, Lakhdar

Belhocine, Ali

Belhout, Samir A.

Belibasakis, George N.

Belic, Domagoj

Beligni, Alessio 
Belikov, Juri

Bell, Brian

Bell, Daniel

Bell, John

Bellaiche, Martine

Bellantone, Vincenzo

Bellekens, Ben

Bellekens, Xavier

Belletti, Beatrice

Bellezza, Ilaria

Belli, Laura

Bellido, Francisco

Bellini, Maria Irene

Bellotto, Maurizio

Belloulata, Kamel

Bellstedt, Peter

Belmonte, Jesús López

Belnoue, Jonathan

Beloiu, Robert

Belousov, Petr

Belousov, Yury M.

Belovich, Joanne M.

Belsak, Ales

Belter, Dominik

Beltowski, Jerzy

Beltrán Novillo, Fernando J.

Beltran, Ana M.

Beltsios, Konstantinos

Belussi, Lorenzo

Belyi, Valiantsin

Bempelou, Eleftheria. D.

Ben Arfi, Anis

Ben Azouz, Aymen

Ben Haj Frej, Mohamed

Ben Hmida, Jalel

Ben Kaab, Sofiene

Benaouicha, Mustapha

Benarba, Bachir

Benard, Emmanuel

Benato, Alberto

Benavente-Peces, César

Benavides, Carmen

Benavides, David

Benbouzid, Mohamed

Benca, Emir

Bencardino, Daniela

Bencardino, Francesco

Bence, Molnár

Bencharit, Sompop

Benda, Jakub

Bender, Kathryn
Bender, Philipp

Bendixsen, Casper

Benedetti, Francesca

Benedic, Fabien

Benedicenti, Luigi

Béni, Szabolcs

Benim, Ali Cemal

Benis, Arriel

Benítez Andrades, Jose Alberto

Benitez-Garcia, Gibran

Benito-Román, Óscar

Beniuga, Razvan

Benkoczi, Robert

Bennamoun, Lyes

Bennardo, Francesco

Bennett, Christopher

Bennett, Grant N.

Ben-Othman, Sana

Bensaid, Samir

Benseman, Timothy

Ben-Tal, Oded

Bentayeb, Fadila

Bentes, Carlos

Beňuš, Radoslav

Benz, Heather

Bera, Gopal

Beranič , Tina

Berardi, Valentino Paolo

Berardo, Alice

Berbrayer, David

Berci, Marco

Beretta, Gabriele

Bereza-Malcolm, Lara

Berezin, Mikhail Y.

Bergamini, Andrea

Bergandi, Loredana

Bergante, Sara

Berger, Rafal

Berger, Walter

Bergold, Sebastian

Bergonzoli, Simone

Berhausen, Sebastian

Beriáin, Andoni

Beris, Antony

Beritelli, Francesco

Berlanga, Rafael

Berlec, Tomaz

Berlin, Shai

Bermejo Higuera, Javier

Bernacki, Bruce

Bernal, Francisco 
Bernard, Benoît

Bernard, Marek K.

Bernardello, Fabio

Bernardi, Sara

Bernardino, Jorge

Bernardo, Luis Filipe Almeida

Bernasconi, Roberto

Bernat, Jakub

Bernat, Przemysław

Bernatavičienè, Jolita

Bernatová, Silvie

Bernhardt, Anne

Bernstein, Joseph

Bernussi, Ayrton

Berrezueta, Edgar

BERRI, Pier Carlo

Berruti, Teresa Maria

Bertaccini, Assunta

Berteau, Jean-Philippe

Bertello, Alberto

Berthod, Patrice

Berthold-Pluta, Anna

Bertini, Leonardo

Berto, Filippo

Bertocci, Francesco

Berton, Federico

Berton, Marco

Bertoncelj, Andrej

Bertsch, Arnaud

Bertz, Alexander

Besalú, Emili

Beskopylny, Alexey

Besliu, Irina

Besnard, Aurélien

Bespalko, Yulia N.

Bessais, Lotfi

Besset, Sebastien

Bessmertny, Igor A.

Bessonov, Vladimir O.

Best, Andrew

Bestak, Robert

Beszédes, Sándor

Betke, Ulf

Betterle, Nico

Betti, Michele

Bettini, Giordana

Betz, Johannes

Betz, Simon

Beu, Dorin

Beugnon, Jerome

Beumer, Rijkelt
Beverly, Levi J.

Bevins, James E.

Beyer, Hans Georg

Beyrer, Michael

Bezirtzoglou, Eugenia

Bezuijen, Adam

Bezzateev, Sergey

Bhagavathula, Rajaram

Bhandari, Khadak Singh

Bhandari, Manohar Prasad

Bhargava, Kriti

Bharti, Pratool

Bhasin, Shivam

Bhaskar, Abhinav

Bhate, Dhruv

Bhatia, Shashi Kant

Bhattacharyya, Mayukh

Bhatti, Bilal Ahmad

Bhatti, Fazal-ur-Rehman

Bhethenabotla, Venkat

Bhounsule, Pranav A.

Bhowmick, Sankha

Bhowmik, Basuraj

Bhowmik, Debsindhu

Bhowmik, Lal Mohan

Bhudolia, Somen K.

Bhuiyan, Ehsan

Bhutta, Muhammad

Bi, Guihong

Bi, Siwen

Bi, Ting

Bia, Pietro

Biagini, Giuseppe

Biagiotti, Luigi

Bialas, Katarzyna

Białek, Marzena

Białobrzeska, Beata

Bian, Sizhen

Biancalana, Valerio

Biancardo, Salvatore Antonio

Bianchi, Giacomo

Bianchi, Giulia

Bianchini, Claudio

Bianco, Angelica

Bianco, Armandodoriano

Bianco, Maria Giovanna

Biancolillo, Alessandra

Bianconi, Francesco

Biasotti, Silvia

Bibbo, Daniele

Bici, Michele 


\begin{tabular}{|c|c|}
\hline Bicout, Dominique & Biondi, Giulio \\
\hline Biegala, Michal & Birbas, Alexios \\
\hline Biel, Carmen & Birdwell, Justin \\
\hline Bielecka, Marzena & Biris, Cristina \\
\hline Bielecki, Andrzej & Birjandi, Anahid Ahmadi \\
\hline Biel-Solé, Domingo & Birk, Andreas \\
\hline Bien, Anna & Birk, Udo \\
\hline Bieniek, Andrzej & Birlescu, Iosif \\
\hline Bienkowski, Jerzy & Birou, Iulian M. T. \\
\hline Bienvenido-Huertas, David & Birowosuto, Muhammad \\
\hline Biernat, Jan & Birowska, Magdalena \\
\hline Bierzuńska, Paulina & Birsa, Mihail Lucian \\
\hline Bies, Alexander J. & Bis, Marta \\
\hline Biessmann, Felix & Biscarini, Andrea \\
\hline Bigaud, David & Bischi, Aldo \\
\hline Biglia, Alessandro & Bischof, Sandra \\
\hline Bignardi, Cristina & Biswas, Asim \\
\hline Bignozzi, Carlo Alberto & Biswas, Manik Chandra \\
\hline Bigourd, Damien & Biswas, Roopa \\
\hline Bijaieh, Mehrzad M. & Biswas, Soumyajyoti \\
\hline Bijle, Mohammed & Biswas, Sumana \\
\hline Bikbaev, Rashid G. & Bitar, Ahmad W. \\
\hline Bikker, Jacco & Bithas, Petros S. \\
\hline Bilal, Muhammad & Bitkowska, Agnieszka \\
\hline Bilan, Yuriy & Bitta, Jan \\
\hline Bilandžija, Nikola & Bitter, Wilbert \\
\hline Bilbao, Daniel & Bittolo Bon, Silvia \\
\hline Bilbao, Javier & Bizhani, Majid \\
\hline Bilén, Sven G. & Bizon, Katarzyna \\
\hline Bilheux, Jean & Bjelland, Henrik \\
\hline Bilican, Doga & Björklund, Christina \\
\hline Bilicki, Sławomir & Blachnik, Marcin \\
\hline Bilík, Petr & Błachowicz, Tomasz \\
\hline Bill, Ralf & Blachowski, Bartlomiej \\
\hline Billah, AHM Muntasir & Blagec, Kathrin \\
\hline Billah, Muntasir & Blagojevic Zagorac, Gordana \\
\hline Billeci, Lucia & Blagojevic, Branimir \\
\hline Billing, Erik A. & Blaimer, Martin \\
\hline Billinghurst, Mark & Blair, Christopher \\
\hline Bin Ahmadon, Mohd Anuaruddin & Blake, David \\
\hline Biňasová, Vladimíra & Blanc, Philippe \\
\hline Binda, Jacek & Blanc, Wilfried \\
\hline Bindoff, Ivan & Blanchard, Denise \\
\hline Bindzarova Gergelova, & Blanche, Pierre-alexandre \\
\hline Binh, Nguyen Ngoc & Blanco Fernandez, Matias \\
\hline Biniak-Pieróg, Małgorzata & Blanco, David \\
\hline Binkowski, Tomasz & Blanco, Juan C. \\
\hline Binzhong, Zhou & Blanco-Salas, José \\
\hline Biocca, Marcello & Blanco-Ward, Daniel \\
\hline Biolzi, Luigi & Blanes, Jorge \\
\hline Biondi, Filippo & Blanloeuil, Philippe \\
\hline
\end{tabular}


Blanpain, Bart

Blaser, Nello

Błasiak, Sławomir

Blaskova, Martina

Blaszczuk, Artur

Błaszczyński, Tomasz

Błaszczyszyn, Monika

Blavette, Anne

Błażejowski, Marcin

Blazevic, David

Blažević, Ivica

Blažič, Boštjan

Blazy, Rafał

Blecharz, Krzysztof

Blecich, Paolo

Blecua, César

Bleiner, Davide

Bleul, Regina

Blinder, David

Blinzler, Brina J.

Block, Jean-Claude

Bloem, Elke

Bloisi, Domenico

Blonski, Slawomir

Bloor, Stephen

Blukacz-Richards, Agnes

Blunt, Warren

Boanca, Paunita

Boarescu, Paul-Mihai

Bobadilha, Gabrielly S.

Bobbo, Sergio

Bobi, Miguel Angel Sanz

Bobinger, Marco

Bobiński, Jerzy

Bobkowska, Katarzyna

Boboc, Marius

Boboc, Răzvan Gabriel

Bobrov, Nikita Yu.

Bobulska, Lenka

Bobulski, Janusz

Bobyr, Maxim

Boccadoro, Pietro

Bocchetta, Patrizia

Bocchi, Leonardo

Bocci, Edoardo

Boccia, Antonella Caterina

Boccignone, Giuseppe

Bocewicz, Grzegorz

Bochenkov, Vladimir

Bocîrnea, Amelia Elena

Bock, Martin
Böckl, Benjamin

Bocko, Jozef

Bocu, Razvan

Bocullo, Vytautas

Bodaghi, Mahdi

Bodar, Charles

Boddaert, Xavier

Bode, Bela Ernest

Bode, Florin

Bodi, Ştefan

Bodisco, Timothy

Bodnar, Jean Luc

Bodzas, Sandor

Boehler, Quentin

Boehm, Daniela

Boehme, Luc

Boekema, Bouke

Boente Lopez, Carlos

Boese, Adrian Daniel

Boffa, Vittorio

Boffito, Monica

Bogataj, Marija

Bogataj, Špela

Bogdan, Heghes

Bogdan, Paul

Bogdanovic, Jelena

Bogdanovskaya, Vera

Bogdanowicz, Krzysztof Artur

Bogenfeld, Raffel

Bogris, Adonis

Boguniewicz-Zablocka, Joanna

Bohannon, Richard W.

Bohdalová, Mária

Bohinc, Klemen

Bohinc, Tanja

Bohl, Douglas G.

Böhm, Michał

Bohrer, Benjamin M.

Bohuslava, Juhasova

Boja, Catalin

Bojić, Mirza

Bokde, Neeraj

Bökler, Fritz

Boland, Francis Morgan

Boland, Sebastian

Bolander, John

Bolbot, Victor

Boldyryev, Stanislav

Bolek, Vladimir

Bolles, André

Bollinger, Lance 
Bollini, Letizia

Bollt, Erik M.

Bolme, Cindy

Bolognani, Silverio

Bolondi, Giorgio

Bölöni, Ladislau

Bolonio, David

Bolotin, Yu V.

Bolotov, Leonid

Bolouki, Nima

Bolshov, Mikhail

Boltersdorf, Jonathan

Bolton, James

Bomantara, Radity

Bombek, Gorazd

Bombelli, Paolo

Bonacich, Phillip

Bonadies, Irene

Bonaglia, Stefano

Bonanomi, Marcella

Bonardi, Fabien

Bonatto, Alexandre

Bonci, Andrea

Bondani, Maria

Bondok, Doaa

Bondorf, Steffen

Bonduà, Stefano

Bondzior, Bartosz

Bone, Gary

Bone, Julia

Bonek, Mirosław S.

Bonelli, Francesco

Bonetta, Silvia

Bongiovanni, Laura

Bonì, Roberta

Böning, Dieter

Bonini, Matteo

Bonkowski, Piotr

Bonora, Nicola

Bonsignore, Carmelo Peter

Bonyár, Attila

Booker, Edward

Boori, Mukesh Singh

Bopp, Fritz W.

Boppana, Bharathi

Boratyńska, Katarzyna

Borboni, Alberto

Borchers, Christine

Bordea, Ioana Roxana

Bordeenithikasem,

Bordel Sánchez, Borja
Bordes, María Dolores Llobat

Bordihn, Stefan

Bordoni, Massimiliano

Borecki, Michal

Borelli, Davide

Borge Diez, David

Borghesi, Andrea

Borghi, Alessandra

Borghini, Andrea

Borgianni, Yuri

Borhan, Md. Saidul

Borisoff, Jaimie

Borisova, Ekaterina

Borkowski, Piotr

Bormann, Richard

Bornmann, Jonas

Böröcz, Péter

Borodianskiy, Konstantin

Boroński, Dariusz

Borota, Ana

Borowczyk, Henryk

Borowiak, Klaudia-Anna

Borowicz, Marcin

Borowik, Agata

Borowska, Marta

Borowska-Stefańska, Marta

Borowski, Marek

Borràs Sol, Júlia

Borrego, Carlos

Borrelli, Luigi

Borri, Simone

Borriello, Anna

Borse, Vikrant

Borst, Jan Willem

Bortkiewicz, Alicja

Bortolotto, Tissiana

Bortolus, Marco

Bortolussi, Silva

Borucka, Anna

Borugadda, Venu Babu

Borusiak, Barbara

Borza, Paul Nicolae

Borzyszkowski, Jacek

Bos, Gerard

Boscato, Giosuè

Boschetti, Giovanni

Boschetto, Alberto

Boschin, Giovanna

Bosco, Giovanni

Boscoboinik, J. Anibal

Bose, Bipasha 
Bose, Rohit

Bosioc, Ilie Alin

Boškoski, Pavle

Boskou, George

Bosman, Conrado A.

Bosnjak, Josipa

Bossu, Maurizio

Bostjancic, Emanuela

Boston, Kevin

Boström, Kim

Botez, Ruxandra

Botlik, Josef

Botoran, Oana

Botrel, Loïc

Botsis, John

Botta, Fabio

Botta, Marco

Bottagisio, Marta

Botte, Marilisa

Bottiglione, Francesco

Bottin, Matteo

Botto, Anna

Botts, Ryan

Botubol-Ares, José

Bouaissi, Aissa

Bouali, El Hachemi

Boubekri, Nourredine

Boucenna, Sofiane

Boucheron, Romuald

Bouchner, Petr

Boudou, Thomas

Bouet, Christel

Bouferguene, Ahmed

Bougdah, Hocine

Bougen-Zhukov, Nicola

Bouhamed, Ayda

Bouitbir, Jamal

Boukhers, Zeyd

Boukid, Fatma

Boulesbaa, Abdelaziz

Boulogeorgos, Alexandros-Apostolos A.

Bouloumpasis, Ioannis

Boumezgane, Oussama

Boumghar, Yacine

Bourdine, Anton V.

Bourgeois, Denis

Bourguet, Salvy

Bourikas, Leonidas

Bournival, Ghislain

Bouroussis, Constantinos A.

Boursianis, Achilles
Bousia, Alexandra

Bousquet, Jean-Francois

Bousson, K.

Boutayeb, Halim

Boutinguiza, Mohamed

Boutsi, Argyro-Maria

Boutteau, Rémi

Bouvier, Yann E.

Bouwer, James C.

Bouza-Rodríguez, José Benito

Bouzid, Allal El Moubarek

Bouzinac, Catherine

Bovino, Fabio Antonio

Bowen, James

Boxman, Raymond L.

Boyang, Su

Boyano, Ana

Boyatzis, Stamatis

Boyd, Douglas

Boyd, Gary

Boyle, Elizabeth

Boymelgreen, Alicia

Boyvalenkov, Peter

Bozalakov, Dimitar

Bozek, Andrzej

Božek, Pavol

Bozek, Piotr

Bozhko, Yulia Yu.

Bozkurt, Selim

Bozomitu, Radu Gabriel

Brabazon, Dermot

Bracciali, Andrea

Bracho, Gabriela

Brackmann, Christian

Bračun, Drago

Brad, Remus

Brad, Stelian

Bradley, Robert S.

Bradshaw, David

Bragada, Jose A.

Bragazza, Luca

Bragazzi, Nicola Luigi

Brągoszewska, Ewa

Braicu, Cornelia

Brailo, Vlaho

Bramanti, Emilia

Brancaccio, Adriana

Brancati, Renato

Brancato, Virginia

Branco, Fernando G.

Branco, Pedro 


\begin{tabular}{|c|c|}
\hline Brand, Alexander & Brkljača, Mia \\
\hline Brandi, Fernando & Brnić, Josip \\
\hline Brando, Giuseppe & Broadhurst, Catherine Leigh \\
\hline Brandus, Catalina-Alice & Brock, Stefan \\
\hline Brank, Bostjan & Broda, Magdalena \\
\hline Brantley, William A. & Brodny, Jarosław \\
\hline Braný, Dušan & Brogan, Joel \\
\hline Brás, Natércia & Brogi, Simone \\
\hline Braselton, James & Bromuri, Stefano \\
\hline Brassat, Katharina & Bronikowski, Michał \\
\hline Bratu, Polidor & Brookes, Tim \\
\hline Braun, Alexander & Brooks, Hadley \\
\hline Braun, Artur & Broszat, Dominik \\
\hline Brauneck, Jens & Brotons, Jose M. \\
\hline Brauner, Edoardo & Brouwer, Anne-Marie \\
\hline Brauss, Daniel & Brovelli, Maria Antonia \\
\hline Bravo, Ignacio & Brown, Geoffrey \\
\hline Bravo, Miguel & Brown, Justin \\
\hline Bravo, Teresa & Brown, Stephen \\
\hline Bravo-Aguilar, María & Brozović, Bojana \\
\hline Brazel, Anthony & Brożyna, Jacek \\
\hline Brčić Karačonji, Irena & Brtník, Bohumil \\
\hline Brcic, David & Bru, David \\
\hline Brcic, Marino & Bruckmann, Corinna \\
\hline Brcic, Mario & Bruel, Dominique \\
\hline Breard, Arnaud & Brugge, Rowena H. \\
\hline Breban, Stefan & Brumen, Boštjan \\
\hline Brechtl, Jamieson & Brumerčík, František \\
\hline Brégier, Frédérique & Brumitt, Jason \\
\hline Brehar, Raluca & Bruncko, Jaroslav \\
\hline Brehm, Martin & Brundage, Cord M. \\
\hline Bren, Urban & Bruneau Jr, Michael \\
\hline Brenišin, Marek & Brunesi, Emanuele \\
\hline Brescia, Massimo & Brunet, Anton Guimerà \\
\hline Brethé, Jean François & Brunet, Christophe \\
\hline Brezočnik, Lucija & Brunetti, Cecilia \\
\hline Briatico-Vangosa, Francesco & Bruni, Renato \\
\hline Briciu, Victor-Alexandru & Brunner, Andreas \\
\hline Bridgwater, Anthony & Bruno, Nazarena \\
\hline Briffaut, Matthieu & Bruno, Silvana \\
\hline Briga-Sá, Ana & Brus, Grzegorz \\
\hline Brígido, Clarisse & Brus, Jan \\
\hline Brinck, Tore & Brusaporci, Stefano \\
\hline Brincker, Rune & Brust, Tarsis \\
\hline Bringuier, Stefan & Brutti, Alessio \\
\hline Brink, Wyger & Bruzda, Joanna \\
\hline Briongos, Samira & Bruzzese, Claudio \\
\hline Brito, Jose Henrique & Bruzzone, Luca \\
\hline Britz, Margaret L. & Bryner, Elliott \\
\hline Brkic Bakaric, Marija & Bryt-Nitarska, Izabela \\
\hline Brkić, Dejan & Brzeska, Joanna \\
\hline
\end{tabular}


Brzezińska, Dorota

Brzóska, Edyta

brzoska, malgorzata michalina

Brzostowski, Krzysztof

Brzychczy, Edyta

Buades Rubio, Jose Maria

Bubnienè, Urtè

Bubola, Marijan

Bucaioni, Alessio

Bucchi, Francesco

Bucci, Rosaria

Buchanan Berumen, Edgar

Buchanan, Derrick Matthew

Buchel, Olha

Buchman, Sasha

Buchmann, Robert

Buchmeister, Borut

Buchowicz, Andrzej

Buchroithner, Armin

Buciakowski, Mariusz

Bučinskas, Vytautas

Buckley, Conor

Buckley, Jeffrey

Buckley, Richard

Buckley, Sue

Bucko, Samuel

Bucolo, Maide

Bucovețchi, Olga Maria Cristina

Bucur, Daniel

Buczyński, Przemysław

Buda, Natalia

Budaszewski, Daniel

Budinski, Vedran

Budwig, Ralph

Budžaki, Sandra

Budzan, Sebastian

Budzevich, Mikalai

Bueno, Emilio

Bueno, Salvador

Bueso, María C.

Buffoni, Lorenzo

Buga, Ana-Maria

Bugajski, Maciej

Bui Khoi, Phan

Bui, Hoa

Bui, Ngoc Kien

BUI, Ngoc-Tam

Bui, Phuc

Buinevich, Mikhail

Buitrago Esquinas, Eva Maria

Buiu, Catalin
Bujan, Julia

Buj-Corral, Irene

Bujňáková, Petra

Bujnowski, Adam

Bujoreanu, Carmen

Bukin, Oleg

Bukoski, Jacob

Bukowska, Joanna

Bukowski, Przemyslaw

Bulai, Georgiana

Bulej, Vladimír

Bulgakova, Nadezhda

Bulgariu, Laura

Bulic, Mladen

Bulic, Neven

Bulic-Jakus, Floriana

Bullas, Alice

Bullough, John

Bultel, Arnaud

Būmanis, Girts

Buń, Paweł

Bunbury, Judith

Bundaleska, Neli

Bundschuh, Ralph A.

Bunge, Christian-Alexander

Bunting, Erin

Buntov, Evgeny

Buraga, Sabin

Buranaj Hoxha, Besmir

Burattini, Laura

Buravkova, Ludmila

Burdett, Robert

Burdin, Vladimir

Burdziakowski, Paweł

Burdzik, Rafał

Bureika, Gintautas

Bures, Miroslav

Bureš, Radovan

Burgdorf, Martin

Burgess, Scott

Burget, Radek

Burget, Radim

Burghardt, Andrzej

Burgholzer, Peter

Burgi, Kenneth

Burgreen, Greg

Burguete, José Luis Vázquez

Burileanu, Corneliu

Burlacu, Adrian

Burlacu, Sorin

Burlakovs, Juris 
Burlikowski, Wojciech

Burmin, Valery Yurievich

Burmistrov, Igor

Burmistrov, Konstantin

Burmistrz, Michal

Burnat, Barbara

Burns, Jonathan

Burokur, Shah Nawaz

Burpo, Fred J.

Burrascano, Pietro

Burri, Samuel

Bursic, Sathya

Buru, Stefan M.

Burugupally, Sindhu Preetham

Bus, Agnieszka

Buscarino, Arturo

Busico, Gianluigi

Busila, Mariana

Busko, Malgorzata

Bustamante, Eliseo

Bustos, Alejandro

Busu, Mihail

Butka, Brian

Butkus, Mindaugas

Butler, Les

Butler, Tiffiny A.

Butnariu, Silviu

Butnaru, Gina Ionela

Butov, Oleg

Butt, Ali M.

Butterworth, Karl

Buttigieg, Sandra C

Butun, Ismail

Buxton, Samuel

Buyle-Bodin, François

Buzatu, Andrei

Buzoianu, Anca

Buzzelli, Marco

Buzzetti, Michela

Buzzetti, Rafaella

Byeon, Haewon

Bykhovsky, Dima

Bykovsky, Alexey Yu

Bylard, Andrew

Bylieva, Daria

Byliński, Hubert

Bylka, Jedrzej

Byun, Gihoon

Byun, Gwi Bo

Byun, Hi-Ryong

Byun, Junghwan
Byun, Soo-Hwan

Byun, Yong-Hoon

Byun, Yung-Cheol

Bzówka, Joanna

Cabaleiro, David

Caballero, Daniel

Caballero, David

Caballero, Olga

Cabboi, Alessandro

Cabeza-Lainez, Jose M.

Cabezón, Rubén

Cabezuelo, Antonio Sarasa

Cabitza, Federico

Cabral Pinto, Marina

Cabrera I Fausto, Ivan

Cabrera, Humberto

Cabrera, Manuel Montenegro

Cabrini, Marina

Cacak-Pietrzak, Grażyna

Cacan, Martin R.

Caccamo, Maria Teresa

Caccianiga, Gianluigi

Cacciola, Francesco

Cacopardo, Ludovica

Cada, Martin

Cadar, Oana

Cadatal-Raduban, Marilou

Cadena, Jose

Caeiro-Rodríguez, Manuel

Caesarendra, Wahyu

Caetano, Elsa

Caetano, Nidia

Cafarelli, Andrea

Cafaro, Francesco

Caffaro, Federica

Cafolla, Daniele

Caggianese, Giuseppe

Caggiano, Antonio

Cagliero, Cecilia Lucia

Cagnoni, Stefano

Cai, Feng

Cai, Jie

Cai, $\mathrm{Wu}$

Cai, Xingyu

Caignaert, Guy

Cairrão, Elisa

Cała, Marek

Calabretta, Cristiano

Calabrò, Roberto

Calado, João M. F.

Calaf Chica, Jose 
Calantropio, Alessio

Calarco, Anna

Calatayud, David G.

Calbimonte, Jean-Paul

Calcan, Andreea

Caldas, Paulo

Caldeirinha, Rafael

Caldeiro Pedreira, Mari Carmen

Calderini, Ornella

Calderisi, Marco

Calderon, Juan M.

Caleiro, António

Calì, Michele

Caliano, Giosue

Calimeri, Francesco

Calin, Vladeanu

Calin-Adrian, Comes

Calín-Sánchez, Ángel

Calisi, Nicola

Callegari, Camilla

Callegari, Massimo

Callejas, Antonio

Callejo, Miguel Ángel Manso

Callery, Patrick S.

Calligaris, Chiara

Callone, Emanuela

Calò, Pietro Giorgio

Calokerinos, Antony C.

Calvello, Mariarosaria

Calvini, Rosalba

Calvo Gordaliza, Ana Isabel

Calvo Rolle, Jose Luis

Calvo, Ana Cristina

Calvo, Conceptión

Calvo, José Ignacio

Calvo, Roque

Calvo-Guirado, José Luis

Calvo-Lobo, César

Camacho, Jorge

Camacho-Alonso, Fabio

Camara, Eduardo Martínez

Camarda, Kyle

Camargo, Samira Esteves Afonso

Camarinha-Matos, Luis M.

Camblor, Miguel A.

Cambou, Bertrand

Cambria, Erik

Camci, Cengiz

Camera, Corrado

Camerlingo, Carlo

Cameron, Simon J. S.
Camin, Bettina

Camiz, Sergio

Camm, Joseph

Cammarata, Antonio

Cammas-Marion, Sandrine

Cammi, Antonio

Campagnolo, Alberto

Campanacci, Laura

Campatelli, Gianni

Campbell, John

Campbell, Michael

Campbell, Sawyer D.

Campi, Cristina

Campiglio, Chiara Emma

Campillo, Natalia

Campioni, Ilaria

Campisi, Giuseppina

Campos Rosa, Joaquín María

Campos Souza, Paulo Vitor

Campos, Armando

Campos, Debora

Campos, Ricard

Campos-Gaona, David

Campos-Martin, Jose M.

Campus, Guglielmo

Canal, José Maria

Canale, Claudio

Canale, Laurent

Canata, Gian Luigi

Canavero, Flavio

Candela, Gustavo

Cándido, Gutierrez Montes

Candolfi, Christophe

Candries, Maxim

Canedo, Edna Dias

Cañete, Francisco J.

Canet-Ferrer, Josep

Canfield, Brian

Cang, Zixuan

Canha, Nuno

Caniato, Marco

Cannarile, Francesco

Cannas, Ignazio

Cannas, Marco

Cannavo, Flavio

Cannistraro, Giuseppe

Cannone Falchetto, Augusto

Cano, Alberto

Cano, Israel Garcia

Cano, Juan Luis

Cano, Juan-Carlos 
Cano, Maria-Dolores

Caño-García, Manuel

Cano-Moreno, Juan David

Canovas Martinez, Rocio

Cansado, Pablo

Canselier, Jean Paul

Cantamessa, Simone

Cantero, Blas

Cantero, Domingo

Cantisani, Giuseppe

Cantizano González, Alexis

Cantoni, Jacopo

Cantor-Cutiva, Lady Catherine

Cantrak, Djordje

Cantzos, Demetrios

Cao, Hoang-Long

Cao, Hung

Cao, Jian

Cao, Lin

Cao, Lizhou

Cao, Rui

Cao, Wen-Ping

Cao, Xin

Cao, Xudong

Cao, Yunqi

Cao, Zhiguang

Capaldo, Pietro

Capece, Nicola

Capel, Manuel I.

Capezza, Antonio

Capilleri, Piera Paola Francesca

Capitani, Renzo

Capitão, Silvino Dias

Capobianco, Enrico

Capobianco, Giuseppe

Capogni, Marco

Capolupo, Alessandra

Capone, Alessandro

Caponnetto, Salvatore

Caporossi, Lidia

Caporusso, Nicholas

Capote, Rene

Capozzi, Luigi Carlo

Capozzi, Vincenzo

Cappai, Francesco

Cappai, Maria Grazia

Capparè, Paolo

Cappelli, Alessio

Cappon, Giacomo

Cappuzzello, Francesco

Capra, Alessandro
Capra, Gian Franco

Capraro, Jessica

Capriotti, Margherita

Capuano, Francesco

Caputo, Fabio

Caputo, Francesco

Cara, Eleonora

Caraballo, Israel

Carabin, Giovanni

Carabineiro, Sónia

Carac, Geta

Caracciolo, Giuseppina

Caraffini, Fabio

Cara-Jiménez, Jorge

Carames, João Manuel Mendes

Caramia, Giovanni

Caramia, Massimiliano

Carapau, Fernando Manuel Lucas

Carballal, Adrian

Carballedo, Roberto

Carbone, Giuseppe

Carbone, Katya

Carbonell Carrera, Carlos

Carboni, Lucia

Carcadea, Elena

Cardaci, Alessio

Cardani, Giuliana

Cardano, Filippo

Carden, Steve

Cárdenas Pérez, Stefany

Cárdenes, Víctor

Cardenia, Vladimiro

Cardeñoso-Payo, Valentin

Cardillo, Elena

Cardon, Ludwig

Cardone, Daniela

Cardoso, Jorge C. S.

Cardoso, Rafael C.

Cardullo, Nunzio

Carette, Jérôme

Carey, Lawrence D

Caridade, Cristina

Caridade, Pedro Jorge Dos Santos Branco

Caridi, Francesco

Čarija, Zoran

Cariou, Christophe

Carl, Timo

Carleer, Robert

Carles Arribas, José Luis

Carletti, Luca

Carlo, Bianchini 
Carlo, Gabriel

Carlos Ferreira, João

Carlson, Daniel F.

Carlucci, Antonio Paolo

Carlucci, Claudia

Carmen Carnero, María

Carmona-Galán, Ricardo

Carneiro, Miguel

Carnesecchi, Francesca

Carnevale Miino, Marco

Carniel, Emanuele

Carnieletto, Laura

Caroppo, Andrea

Carosio, Federico

Carota, Eleonora

Carotenuto, Gianfranco

Carotenuto, Riccardo

Carou, Diego

Carozza, Antonio

Carpenter, Joseph P.

Carpenter, Kristin

Carpentieri, Andrea

Carpio, Antonio José

Carr, Anna

Carr, Tim

Carra, Damiano

Carradori, Simone

Carraro, Massimo

Carrasco, Alejandro

Carratù, Marco

Carrera Fernández, Ceferino

Carrera, Carlos Carbonell

Carretero, Luis

Carrieri, Antonio

Carrilho, Eunice

Carrillo De Gea, Juan Manuel

Carrouel, Florence

Carter, Alan

Carter, John D.

Cartmill, Donita L.

Carús, Luis

Caruso, Giandomenico

Caruso, Giulia

Caruso, Matteo

Carvalho, Filipe

Carvalho, José Beleza

Casadei, Roberto

Casado, Alejandro

Casado, Natalia

Casado, Rafael

Casado-Belmonte, María Del Pilar
Casado-Coterillo, Clara

Casado-Hernández, Israel

Casado-Vara, Roberto

Casagrande, Daniele

Casakin, Hernan

Casalino, Gabriella

Casalotti, Arnaldo

Casanueva Marenco, Maria Jose

Casarejos, Enrique

Casas Rius, Joan Ramon

Cascajo, Alberto

Cascardi, Alessio

Cásedas, Guillermo

Casella, Patrizia

Caselles, Oriol

Caserio, Carlo

Casertano, Marcello

Casey, Jennifer

Casiraghi, Elena

Casolo, Siro

Caspary, Reinhard

Casqueiro, Carlos

Cassagnau, Philippe

Cassani, Raymundo

Cassano, Domenico

Cassé, Vincent

Cassidy, Tracy

Cassone, Giuseppe

Castagnetti, Cristina

Castaings, Michel

Castaldi, Giuseppe

Castaldo, Anna

Castañeda, Arkaitz

Castañeda, Carmen

Castaño, Fernando

Casteleiro-Roca, Jose Luis

Castell, Pere

Castellano Román, Manuel

Castellano, Anna

Castellano, Carlo

Castellano, Davide

Castellanos Regalado, Francisco José

Castellari, Massimo

Castellazzi, Giovanni

Castelli, Eric

Castelli, Ivano E.

Castellini, Elena

Castellini, Mirko

Casteren, Jan Van

Castiglione, Arcangelo

Castillo Olea, Cristian 
Castillo Sequera, José Luis

Castillo, Daniel

Castillo, Pedro A.

Castillo, Tony

Castillo-Ruiz, Francisco J.

Castiñeiras, Pedro

Castro Esteves, Pedro José

Castro, Ana Meria

Castro, Andre

Castro, Enrique

Castro, Jesús

Castro, John W.

Castro, José Javier Fernández

Castro, José Luis Alba

Castro, José Raúl

Castro-Fresno, Daniel

Castronuovo, Donato

Casu, Gavino

Casuso-Holgado, Maria Jesus

Catak, Ferhat Ozgur

Catalano, Agostino

Catalano, Antonino

Catalano, Enrico

Catalano, Giuseppe

Catalano, Pasquale

Catalfamo, Paola

Catalin, Bogdan

Catalin, Dumitrescu

Catalin, Popescu

Catalina, Mirela

Catalina-Lucia, Cocianu

Catani, Martina

Catania, Alessandro

Catapano, Anita

Catarina Paz, Maria

Catarino, Susana

Catedra, Felipe

Catelani, Tiziano

Catena, Robert

Caterino, Nicola

Cattan, Grégoire

Cattaneo, Sara

Cattani, Carlo

Cattano, Giulio

Cattò, Cristina

Catunescu, Giorgiana

Catuogno, Luigi

Caudano, Yves

Causa, Flavia

Causer, Grace

Cautela, Domenico
Cava, Claudia

Cavacas, Maria Alzira

Cavaco Mendes, Mário José Gonçalves

Cavagnini, Rossana

Cavalaris, Chris

Cavaleri, Liborio

Cavalieri, Francesco

Cavallaro, Antonio

Cavalli, Rosa

Cavallo, Eugenio

Cavas-Martínez, Francisco

Cavenaghi, Marcos

Caydamli, Yavuz

Cazacu, Constantin

Cazacu, Emil

Cazan, Cristina

Cazenille, Leo

Cazorla, Miguel

Cazzola, Dario

Cazzola, Roberta

Ceballos-Laita, Luis

Cebrián, Daniel Rivero

Ceburnis, Darius

Cecchi, Stefania

Ceccone, Giacomo

Čech, Jaroslav

Čech, Martin

Cechowicz, Radosław

Cecilia, Hodur

Cecrdle, Jiri

Cedervall, Johan

Cefalo, Raffaela

Cehlin, Mathias

Celadyn, Magdalena

Celaya, Elisabete Alberdi

Celdrán Bernabeu, Marco A.

Celentano, Laura

Celi, Simona

Celik Girgin, Sinem

Celli, Fabio

Celona, Luigi

Cely, Juan S.

Čènas, Narimantas

Cenek, Martin

Cenký, Matej

Centis, Rosella

Centonze, Diego

Cep, Robert

Ceran, Bartosz

Ceraolo, Massimo

Cerasuolo, Giovanni 
Cerbu, Camelia

Cerciello, Francesca

Cerdà-Alabern, Llorenç

Ceresoli, Davide

Cerezo, Javier

Cerezo-Narváez, Alberto

Cerjan, Ben

Čermák, Martin

Cermeno, Maria

Černohorský, Josef

Cerny, Tomas

Ceron-Carrasco, Jose

Cerqueiro-Pequeño, Jorge

Cerra, Bruno

Cerrato, Andrea

Cerro, Gianni

Cerrone, Federico

Cerrone, Margherita

Cerroni, Daniele

Cerruto, Emanuele

Ceruti, Alessandro

Cervera-Garvi, Pablo

Cervi, Eric

Cervino, Gabriele

Cesário, Rute

Cesarz-Andraczke, Katarzyna

Cesmeci, Sevki

Česnavičius, Ramūnas

Česnulevičius, Algimantas

Česonienè, Laima

Cevoli, Chiara

Ceylan, Hakan

Cha, Hyeongyun

Cha, Hyo Soung

Cha, Sang-Ryul

Cha, Wonsuk

Cha, Young-Jin

Cha, Youngsu

Chaaban, Hala

Chaberek-Karwacka, Grazyna

Chablat, Damien Charles

Chacko, Jenu Varghese

Chacon, Rolando

Chaczykowski, Maciej

Chadli, Mohammed

Chae, Bongsug

Chae, Gyu-Soo

Chae, Junjae

Chai, Kyu Yun

Chai, Rifai

Chai, Sangmi
Chai, Wei

Chai, Young Ho

CHAIX, Jean-François

Chakrabarti, Amartya

Chakraborti, Rajat

Chakraborty, Goutam

Chakraborty, Joyraj

Chakraborty, Nilay

Chakrapani, Sunil

Chakravarty, Shatadru

Chalfant, Julie

Chalikias, Miltiadis

Chalioris, Constantin

Chalker-Scott, Linda

Chalmers, Jeffrey John

Chalmeta, Ricardo

Chalov, Sergey

Chamorro-Trenado, Miquel Àngel

Chamoso Santos, Pablo

Chan, Alan

Chan, Clement

Chan, Dave

Chan, Edward

Chan, Hon Fai

Chan, Joseph Chang Lun

Chan, Ka Lung Andrew

Chan, Kwok-Ping

Chan, Puthearath

Chan, Shu Ning

Chan, Teck Kai

Chanda, Avishek

Chandar, Dominic

Chandra, Daryus

Chandra, Subhash

Chandramouli, Kulshreshtha

Chang, Che-Jung

Chang, Cheng-Yuan

Chang, Chia-Chen

CHANG, Chia-Ming

Chang, Choong-Koo

Chang, Der-Wen

Chang, Fuh-Yu

Chang, Han-Chao

Chang, Hsiao-Yun

Chang, IPin

Chang, Jeang-Lin

Chang, Jen-Yuan (James)

Chang, Jongwha

Chang, Jui-Hung

Chang, Jui-Yung

Chang, Ke-Vin 
Chang, Kuo-Jen

Chang, Lin-huang

Chang, Marvin

Chang, Pearl Chun

Chang, Shih-Ying

Chang, Shu-Chun

Chang, Teng-Wen

Chang, Wanjung

Chang, Wenlong

Chang, Wonkeun

Chang, Yan

Chang, Yau-Zen

Chang, Yeon S.

Chang, Yeong-Hwa

Chang, Young Ki

Chang, Yuan-Jen

Chang, Yue-Shan

Chang, Yuh-Shihng

Chang, Yuhua

Changaival, Boonyarit

Chantana, Jakapan

Chanudet, Vincent

Chao, Chia-Hsin

Chao, Ou-yang

Chaouki, Hicham

Chapman, Andrew John

Chapman, Christian D.

Chapman, Pippa

Chapman, Ryan

Charalampakis, Marinos

Charisiou, Nikolaos

Charissis, Vassilis

Charitidis, Costas A.

Charmpis, Dimos C.

Charpentier, Ludovic

Charpin, Laurent

Charvat, Karel

Charvátová, Hana

Chasalevris, Athanasios

Chataigner, Sylvain

Chataut, Robin

Chatterjee, Prosenjit

Chatterjee, Subhasri

Chatterjee, Tanmoy

Chatterley, Adam S

Chattha, Tariq

Chaturvedi, Iti

Chatzakis, John

Chatzilari, Elisavet

Chatzistamatis, Stamatis

Chatzitheodoridis, Fotios
Chatzopoulou, Paschalina

Chau, Kwok-wing

Chaubey, Saurabh

Chaudet, Claude

Chaudhary, Ankit

Chaudhry, Muhammad Umar

Chaudhry, Rasul

Chaudhuri, Arunima

Chauhan, Munish

Chauhan, Vedang Dilipkumar

Chava, Rama Krishna

Chaves, Deisy

Chawla, Ramesh

Che, Jiaxing

Che, Yuzhang

Cheah, Sin-Mei

Checchi, Vittorio

Chechulin, Andrey

Chechurin, Leonid

Cheer, Jordan

Chegaev, Kostantin

Chegenizadeh, Amin

Chehri, Abdellah

Chehroudi, Babak

Chekurov, Sergei

Chelariu, Oana-Elena

Chelaru, Teodor-Viorel

Chellasamy, Menaka

Chellini, Giuseppe

Chen, Aoyu

Chen, Bo

Chen, Boli

Chen, Bolin

Chen, Che

Chen, Cheng-Wei

Chen, Cheng-Chih

Chen, Chiachung

Chen, Chia-Yu

Chen, Chien-Chang

Chen, Chien-Sheng

Chen, Chih-Chiang

Chen, Chih-Hsuan

Chen, Ching-Nuo

Chen, Chin-Ling

Chen, Chin-Shan

Chen, Chin-Sheng

Chen, Chin-Tai

Chen, Chiung Yao

Chen, Chun

Chen, Chung-Chuan

Chen, Chung-Hwan 
Chen, Da-Ren

Chen, Dechan

Chen, Dejiu

Chen, Dyi-Cheng

Chen, Feng

Chen, Guoxun

Chen, Hailan

Chen, Hanchi

Chen, Hang

Chen, Hansen

Chen, Hone-Zern

Chen, Hongtian

Chen, Houyang

Chen, Hsiang-Chieh

Chen, Hsiang-Yin

Chen, Hsuan-Chu

Chen, Huang-Ming

Chen, Huei-Yen Winnie

Chen, Hui

Chen, Huizhi

Chen, Hung-Yu

Chen, Jer-Ming

Chen, Jiahong

Chen, Jianjun

Chen, Jia-Shiun

Chen, Ju-Chin

Chen, Jun

Chen, Ke

Chen, Keren

Chen, Kuan-Chung

Chen, Kuan-Fu

Chen, Kuang-Hua

Chen, Kuen-Suan

Chen, Leo

Chen, Liang-Bi

Chen, Liang-Yu

Chen, Lin

Chen, Liwen

Chen, Lung-Chien

Chen, Mao

Chen, Miaw-Ling

Chen, Mingchih

Chen, Mingtao

Chen, Mingzhe

Chen, Mingzhou

Chen, Nai-Hua

Chen, Nan

Chen, Pei-Ching

Chen, Po-Tuan

Chen, Po-Wen

Chen, Qiao-Hong
Chen, Ruiyao

Chen, Shengen

Chen, Shih-Chih

Chen, Shih-Yun

Chen, Shing-Jye

Chen, Shukun

Chen, Shun-Hsing

Chen, Shuo

Chen, Shuo-Han

Chen, Ssu-Han

Chen, Su-Yen

Chen, Suzie

Chen, Szu-Ying

Chen, Tai-Heng

Chen, Tao

Chen, Tei-Chen

Chen, $\mathrm{Ti}$

Chen, Tianhua

Chen, Tsung-Chia

Chen, Wei-Bo

Chen, Weijian

Chen, Wei-Ting

Chen, Wen-Hsiang

Chen, $\mathrm{Wu}$

Chen, Xianzhong

Chen, Xiaoxin

Chen, Xiaoyong

Chen, Xin

Chen, Xiyuan

Chen, Xuemin

Chen, Y.-Hui

Chen, Yawen

Chen, Ye-Hwa

Chen, Yenming J.

Chen, Yeou-Jiunn

Chen, Yi-Chin

Chen, Yi-Chung

Chen, Yingnong

Chen, Yi-Tui

Chen, Yi-Wen

Chen, Yizheng

Chen, $\mathrm{Yu}$

Chen, Yuan-Chien

Chen, Yuan-Ho

Chen, Yue

Chen, Yu-Hsuan

Chen, Yu-Jen

Chen, Yulin

Chen, Yung Yue

Chen, Yung-Sheng

Chen, Yuting 
Chen, Yuwen

Chen, Zengshun

Chen, Zeyu

Chen, Zhao

Chen, Zhe

Chen, Zhen

Chen, Zhichao

Chen, Zhitong

Chen, Zi

Chendev, Yury

Cheneler, David

Cheng, Bai-You

Cheng, Chia-Chi

Cheng, Chia-Hsin

Cheng, Chih-Chia

Cheng, Chin-Chi

Cheng, Chin-hsing

Cheng, Eric Ka-wai

Cheng, Fang

Cheng, Francis

Cheng, HaoYang

Cheng, Hsin-Ming

Cheng, Huanyu (Larry)

Cheng, Irene

Cheng, Jiang

Cheng, Jie

Cheng, Meng-Chun

Cheng, Qingqing

Cheng, Shing Shin

Cheng, Szu-Cheng

Cheng, Tsang-Hsiang

Cheng, Wen-chieh

Cheng, Yu-Chieh

Cheng, Yufang

Cheng, Yu-Huei

Cheng, Yu-Shan

Cheon, Jason

Cheong, Cheolung

Cheong, Kang Hao

Cheong, Mi Sun

Cheong, Taesu

Cheong, Yuen-Ki

Chepinskiy, Sergey A.

Cheraghi Bidsorkhi, Hossein

Cheraghi, Seyed Ali

Cheraghian, Goshtasp

Cherian, Chinchu

Cherifi, Karim

Cherkaoui, Karim

Chernyi, Sergei

Chernyshov, Mikhail
Cherrie, John

Cherubini, Stefania

Cherukuru, Nihanth W.

Chesi, Giovanni

Cheskis, Dima

Chessa, Manuela

Cheung, Angus K. F.

Chevallier, Julien

Chew, Chengzi

Chew, Nick Guan Pin

Chhatwani, Sachin

Chi, Celestine N.

Chi, Hung-Lin

Chi, Po-Wen

Chiacchio, Ferdinando

Chiadighikaobi, Paschal Chimeremeze

Chiandone, Massimiliano

Chianella, Iva

Chianese, Simeone

Chiang, Cheng-Tien

Chiang, Chen-Kuo

Chiang, Hai-Pang

Chiang, Ming-Hsi

Chiang, Shiuh-hua Wood

Chiang, Shou-Hao

Chiang, Yi-Ting

Chiang, Yu-Chung

Chiappone, Annalisa

Chiara, Focaccetti

Chiaradonna, Anna

Chiaramello, Emma

Chiarini, Luigi

Chiasera, Alessandro

Chiba, Kazuhisa

Chibac-Scutaru, Andreea L.

Chicardi, Ernesto

CHICEA, Dan

Chicet, Daniela

Chicos, Lucia-Antoneta

Chieffo, Nicola

Chiementin, Xavier

Chien, Shih-Chang

Chien, Ying-Ren

Chien, Yu-Hung

Chih, Mingchang

Chikanori, Hashimoto

Chimenos, Josep Maria

Chin, Koo Bok

Chinello, Francesco

Ching, Tak-Shing

Chinnam, Parameswara Rao 
Chinnici, Fabio

Chinthammit, Winyu

Chioncel, Cristian Paul

Chiou, Tai-Ying

Chipara, Mircea

Chirico, Giovanni

Chirnside, Anastasia E. M.

Chis, Vasile

Chiu, Chien-Kuo

Chiu, Chuang-Yuan

Chiu, Justin N.W.

Chiu, Po-Sheng

Chiu, Sheng-Yi

Chiu, Yen-Yu

Chiulan, Ioana

Chivelet, Nuria Martín

Chizzola, Remigius

Chladek, Grzegorz

Chli, Maria

Chlond, Bastian

Chlubek, Dariusz

Chmelík, Vojtech

Chmelko, V.

Chmiel, Marta

Chmielarz, Paweł

Chmielewski, Adrian

Chmielewski, Łukasz

CHMIELINSKI, Pawel

Chmyrov, Andriy

Cho, Byung Jin

Cho, Chihyun

Cho, Chunhee

Cho, Dang-il

Cho, Deok-Yong

Cho, Eun-Chel

Cho, Gihwan

Cho, Guangsup

Cho, Honghyun

Cho, Hwan-Gue

Cho, Hyunah

Cho, Hyun-Jae

Cho, In Sun

Cho, Jeongho

Cho, Jong-Rae

Cho, Kuk

Cho, Namchul

Cho, Sang Wan

Cho, Soohyun

Cho, Sooyeon

Cho, Sunghye

Cho, Won-Ik
Cho, Yongyeon

Cho, Young Sik

Cho, Youngho

Chocron, Olivier

Chodakowska, Ewa

Chodorow, Urszula

Choe, Sangho

Choenni, Sunil

Choi, Bong Jun

Choi, Byungjoo

Choi, Chang-Beom

Choi, Doo-Hyun

Choi, Duk-Yong

Choi, Hojong

Choi, Jaeho

Choi, Jaewon

Choi, Jaeyoung

Choi, Jee Woong

Choi, Jongmin

Choi, Sejin

Choi, So Yeong

Choi, Sung Woong

Choi, Sung-Hwan

Choi, Sungyeol

Choi, Sungyun

Choi, Wing Shan

Choi, Woo June

Choi, Woo-Seok

Choi, Wooyeol

Choi, Woo-Young

Choi, Yeung Joon

Choi, Yong Jun

Choi, Yongho

Choi, Yongki

Choi, Youkyung H.

Choi, Youngchol

Choi, Youngjin

Choi, Young-Seok

Choiński, Dariusz

Chojnacki, Jerzy

Cholewinski, Grzegorz

Chollet, Jean-François

Chomette, Baptiste

Chong, Alvin

Chong, Juang-Horng

Chong, Tze Pei

Choo, Pei Ling

Choroszucho, Agnieszka

Chorti, Arsenia (Ersi)

Chorzepa, Mi G.

Chotěborský, Rostislav 
Chotorlishvili, Levan

Chou, Cheng-Tung

Chou, Ching-Shan

Chou, Jerry

Chou, Tien-Yin

Chou, Tzu-Chieh

Chou, Wen-Hai

Choudhuri, Avik

Choudhury, Nazim

Chouhan, Raghuraj Singh

Chouyyok, Wilaiwan

Chow, Desmond

Chow, Hsueh-wen

Chow, James

Chow, Wan-ki

Chowdhury, Abu Sayed

Chowdhury, Niaz

Chowdhury, Piyali

Chowdhury, Rezaul

Chowdhury, Subir

Chrcanovic, Bruno

Chrenko, Daniela

Chrissafis, Konstantinos

Christelis, Vasileios

Christiansen, Christian Kim

Christodoulou, Klitos

Christodoulou, Maria-Ioanna

Christodoulou, Panayiotis

Christof, Lüpkes

Christoforides, Elias

Christoforidis, Georgios

Christophe, Sauvey

Christova, Magdalena

Chronopoulos, Dimitrios

Chronopoulos, Spyridon K.

Chrostowski, Piotr

Chrpa, Lukas

Chrysanthakopoulos, Nikolaos A.

Chrysikou, Loukia

Chrzanowski, Łukasz

Chu, Seok Jae

$\mathrm{Chu}$, Shu-Chuan

Chuah, Yew Khoy

Chuaicham, Chitiphon

Chuang, Cheng-Hung

Chuang, Chiashain

Chuang, Hung-Yi

Chuang, Koay Kuan

Chuang, Tien-Juei

Chuang, Yao Li

Chudzikiewicz, Andrzej
Chui, Hsiang-Chen

CHUI, Kwok Tai

Chul Wee, Lee

Chulkova, Tatiana G.

Chun, Junho

Chun, Pang-jo

Chun, Sejong

Chung, Chih-Chung

Chung, Ching-Hu

Chung, Gehoon

Chung, Hyun-Joong

Chung, Jae-Young

Chung, Joaquin

Chung, Joon

Chung, Kung-Ming

Chung, Meng Ting

Chung, Sheng-Heng

Chung, Young Mo

Chung, Yu-Chi

Chusuei, Charles

Chwała, Marcin

Chwastowski, Jarosław

Chwil, Mirosława

Chworos, Arkadiusz

Chybowski, Leszek

Chycki, Jakub

Chylek, Petr

Chylinski, Filip

Ciabattoni, Lucio

Ciaburro, Giuseppe

Ciacci, Nagaia

Ciampi, Giovanni

Ciancarini, Paolo

Cianci, Pasquale

Cianciulli, Antonia

Cianetti, Filippo

Ciani, Adriano

Ciapessoni, Emanuele

Ciaramella, Angelo

Ciarapica, Filippo Emanuele

Ciardelli, Francesco

Ciarmiello, Loredana

Ciatto, Giovanni

Ciazela, Jakub

Čibej, Uroš

Cicalini, Ilaria

Cicchetti, Renato

Cicchi, Stefano

Cicciù, Marco

Ciccone, Marco

Cicconi, Paolo 
Ciccoritti, Roberto

Ciccotti, Giovanni

Cicea, Claudiu

Cicero, Sergio

Cichomski, Michal

Cichowska, Joanna

Cichowska-Kopczyńska, Iwona

Cichy, Ireneusz

Cicone, Antonio

Cid, Clément

Cid-Pastor, Angel

Ciecholewski, Marcin

Cienfuegos-Suárez, Pablo

Cieplak, Tomasz

Cieslak, Jérôme

Cięszczyk, Sławomir

Cifredo-Chacón, María-Ángeles

Cifuentes, Hector

Cigić, Blaž

Cigrovski, Vjekoslav

Cimato, Stelvio

Cimermanová, Ivana

Cîmpean, Anişoara

Cimurs, Reinis

Cinefra, Maria

Cinta Pinzaru, Simona

Cioccolanti, Luca

Ciocoiu, Robert Catalin

Ciofi, Carmine

Cioncolini, Andrea

Cione, Erika

Ciopec, Mihaela

Ciorap, Radu

Ciortan, Sorin

Ciotlăus, Mădălina

Ciotonea, Carmen

Cipin, Radoslav

Ciprian, Dalibor

Ciraolo, Margeaux

Circiumaru, Adrian

Ciria, Miguel

Ciriaco, Fulvio

Ciriaco, Paola

Cirillo, Marco

Cirillo, Nicola

Cirlugea, Mihaela

Cirovic, Goran

Cirri, Damiano

Cirstea, Stefan

Cirtoaje, Cristina

Cisek, Marcin
Ciski, Mateusz

Cislaghi, Alessio

Cismaşiu, Ildi

Cissell, Jordan R.

Cisterna, Pedro

Ciszak, Olaf

Ciszewski, Mateusz

Ciszkiewicz, Adam

Citroni, Rocco

Ciuc, Mihai

Ciuffoletti, Augusto

Ciupe, Valentin

Ciuprina, Gabriela

Ciura, Krzesimir

Civera, Marco

Civit-Balcells, Anton

Cizdziel, James

Cizek, Jan

Cizelj, Leon

Cizeron, Antoine

Claesen, Luc

Claeys, Kurt

Claireau, Fabien

Clairmont, Lindsey

Clanchy, Felix

Cłapa, Tomasz

Clark, Anthony

Clarke, Neil

Clarke, Samuel D

Class, Holger

Classen, John J.

Claudel, Julien

Claudi, Riccardo

Clausen, Brigitte

Claveau-Mallet, Dominique

Claver, Jose M.

Clemen, Thomas

Clement, Yuen

Clemente, Miguel Pais

Clemente-Suarez, Vincente Javier

Clementi, Catia

Climente-Alarcon, Vicente

Climent-Pérez, Pau

Clinci, Catalin

Clottes, Jean

Cloutier, Robert

Cmiljanic, Nikola

Coakley, Eoin

Coates, Adam

Coates, Stephen

Coban, Oksana 
Coban, Sophia Bethany

Cobianu, Cornel

Cobo Ortega, Ángel

Coca, Eugen

Coccia, Gianluca

Coccia, Mario

Cocheci, Laura

Cocho-Bermejo, Ana

Cochran, Sandy

Coclite, Alessandro

Coclite, Anna Maria

Cocuzza, Silvio

Codetta Raiteri, Daniele

Codina, Esteban

Codrean, Cosmin

Coelho, Antonio

Coelho, João

Coelho, Luis

Coelho, Paulo

Coenen, Jan Willem

Coffey, Niamh

Cogato, Alessia

Coghlan, Niall

Cognetti, Marco

Cohen, Asaf

Cohen, Eliahu

Cohen, Rami

Cohn, Keith

Cohn, Robert

Cojocaru, Florina

Cojocaru, Vasile

Čoko, Duje

Colace, Francesco

Colafranceschi, Stefano

Colajanni, Piero

Colangelo, Francesco

Colangelo, Gianpiero

Colantuoni, Antonio

Coldea, Teodora Emilia

Cole, Jennifer

Cole, Nerida

Coleman, Thomas F.

Coletta, Antonio

Coletti, Chiara

Coletti, Mark A.

Colhon, Mihaela

Colla, Valentina

Collart-Dutilleul, Pierre-Yves

Collen, Anastasija

Colli, Alejandro Nicolás

Collini, Luca
Collins, Frank

Collins, Karen

Collins, Keri

Collins, Tim

Collivignarelli, Maria Cristina

Collmann, Ralph

Collura, Giorgio

Colom, Xavier

Coloma, Juan Francisco

Colombi, John

Colombi, Pierluigi

Colombo, Fabio Maria

Colombo, Federico

Colombo, Luigi Pietro Maria

Colomer Mendoza, Francisco Jose

Colomo-Palacios, Ricardo

Coloretti, Fabio

Colosi, Horațiu Alexandru

Coluccia, Angelo

Coman, Claudiu

Coman, Vasile

Comba, Lorenzo

Combarros Fuertes, Patricia

Combes, Didier

Comes, Calin-Adrian

Comesaña, Rafael

Comesaña-Campos, Alberto

Comez, Lucia

Comi, Giuseppe

Comino, Penny

Commerell, Walter

Commodore, Sarah

Conan, Denis

Concari, Carlo

Conceição, Raquel

Concezzi, Moreno

Concilio, Antonio

Concu, Giovanna

Concu, Riccardo

Condello, Annette

Condino, Sara

Condoret, Jean-Stéphane

Conesa, David

Conforti, Massimo

Confuorto, Pierluigi

Congedo, Paolo Maria

Coni, Mauro

Conibear, Luke

Čonka, Zsolt

Conklin, Darrell

Conley, Jamison 
Connolly, James

Conserva, Enrico

Consilvio, Alice

Consoli, Antonio

Consolino, Lorena

Conson, Massimiliano

Constanta Zoie, Radulescu

Constantinescu, Dan Mihai

Constantinescu, Horia

Constantinescu, Radu

Constantinescu-Aruxandei, Diana

Constantinou, Costas S.

Consumi, Marco

Conte, Davide

Conteduca, Donato

Contessi Negrini, Nicola

Contestabile, Giampiero

Conti, Allegra

Conti, Daniela

Conti, Leonardo

Conti, Michele

Conti, Pio

Conti, Riccardo

Conti, Vincenzo

Contrafatto, Loredana

Contreras, Diana

Convertino, Matteo

Cooke, Michael

Cooklev, Todor

Coop, Matthew

Cooper, Arthur J. L.

Cooper, Matthew G.

Cooper, Robin

Cooper, Steven

Copolovici, Dana Maria

Copot, Cosmin

Coppedè, Fabio

Coppola, Bartolomeo

Coppola, Gennaro

Coppola, Sara

Coratella, Giuseppe

Corbee, Ronald Jan

Corcione, Carola Esposito

Córcoles-Tendero, Juan I.

Corcoran, Peter

Corda, Andrea

Cordaro, Luigi

Cordaro, Massimo

Cordoba, Ricardo

Cordoba-Diaz, Damián

Corigliano, Orlando
Corina, Barbalata

Corizzo, Roberto

Cormack, Alastair N.

Cormos, Ana-Maria

Cornaro, Cristina

Cornelis, François

Cornelius, Thomas Walter

Coros, Maria

Čorović, Selma

Corpino, Riccardo

Corradi, Marco

Corrado, Rindone

Corral Abad, Eduardo

Corral Bobadilla, Marina

Corrales Ramon,

Corrao, Rossella

Correia Caeiro, Catia

Correia, Americo

Correia, Ana Cristina

Correia, António A.

Correia, José A. F. O.

Correia, Luís

Correia, Pedro D. Frazão

Correia, Pedro Miguel Alves Ribeiro

Corres Sanz, Jesus Maria

Corridore, Denise

Corron, Ned J.

Corsaro, Carmelo

Cortaberria, Galder

Cortés, Ulises

Cortese, Lorenzo.

Cortiços, Nuno D.

Cortina Gil, Eduardo

Corves, Burkhard

Cosanti, Barbara

Cosco, Francesco

Coscueta, Ezequiel

Cosentino, Luigi

Cosimbescu, Lelia

Cosma, Pinalysa

Cosme, Fernanda

Cosoiu, Costin Ioan

Cosoli, Gloria

Cossel, Kevin

Cossignani, Lina

Costa, Aldo

Costa, Aniko

Costa, Emanuele

Costa, José Arnaldo Santana

Costa, Maria Do Céu

Costa, Nuno Ricardo 
Costa, Rémi

Costa, Stefania

Costa, Susana

Costa, Tiago

Costa-Castelló, Ramon

Costantino, Domenica

Costanza, Girolamo

Costanzo, Carmelina

Costa-Pinto, Ana Rita

Costa-Requena, Jose

Costas, Andreea

Coste, Jérôme

Costea, Monica

Costea, Teodora

Costel, Plescan

Costello, Robert

Costin, Aaron

Costin, Andrei

Cota, Giuseppe

Cotârleț, Mihaela

Cotas, Carla

Coto, Eliecer

Cotoi, Laura

Coto-Jiménez, Marvin

Cottafava, Dario

Cottone, Giulio

Couchot, Jean-François

Coulibaly, Saliya

Counselman-Carpenter, Elisabeth

Couque, Hervé

Courtney, Jane

Courty, Pierre-Emmanuel

Coustou, Anthony

Coutinho, Joana T.

Coutinho, Mathilda

Couto, António

Couto, Carlos

Couzinet-Mossion, Aurelie

Cova, Tânia

Covarrubias, Mario

Covatariu, Gabriela

Coviello, Giuseppe

Cowling, Michael A.

Cozzella, Lorenzo

Cozzolino, Daniel

Cozzolino, Eugenio

Cozzolino, Marilena

Craciunescu, Aurelian

Craciunescu, Razvan

Craig, Duncan

Crane, Nathan
Craus, Mitică

Craveiro, Rita

Cravero, Carlo

Crecchio, Carmine

Cremonesi, Massimiliano

Cremonini, Marco

CRENGANIS, MIHAI

Crenna, Francesco

Črepinšek, Matej

Crespi Llorens, Damian

Crespi, Pietro

Crespin, Benoît

Crespo Cabillo, Isabel

Crespo Mariño, Juan Luis

Crespo, Rubén González

Cretoiu, Dragos

Cretu, Mihaela

Cretu, Spiridon

Crippa, Paolo

Crisan, Andrei

Crisan-Vida, Mihaela Marcella

Crisp, Ryan W.

Crispo, Fabiana

Cristani, Matteo

Cristian, Dumitru

Cristian, Grava

Cristian-Ioan, Leahu

Cristina, Gaitan Nicoleta

Cristini, Valentina

Cristofaro, Maria Giulia

Cristofori, Davide

Critello, Davide C.

Croce, Anna Cleta

Croce, Pietro

Crochet, Pascale

Crognale, Silvia

Croitorescu, Valerian

Cronin-Golomb, Mark

Crosman, Erik

Cross, Stephen A.

Crossland, Andrew F.

Crotti, Elena

Crowley, James

Crucho, João

Crupi, Giovanni

Crupi, Pasquale

Crupi, Vincenzo

Cruz, Carlos

Cruz, Edmanuel

Cruz, Francisco

Cruz, Rui M. S. 
Cruz, Sandra

Cruz-Chamorro, Ivan

Csámpai, Antal

Csanádi, József

Csató, Lehel

Csiha, Csilla

Csík, Attila

Csirmaz, Laszlo

Csoka, Levente

Csonka, Bálint

Csordás, Anita

Cuadra, Giancarlo

Cuadrado, David Gonzalez

Cuan-Urquizo, Enrique

Cubellis, Maria

Cuberos, Ramón Chacón

Cucciniello, Raffaele

Cuchí-Oterino, José

Cucinotta, Filippo

Cuckov, Filip

Cuculić, Aleksandar

Cuculić, Vlado

Cuculo, Vittorio

Cudziło, Stanisław

Cuellar, Sara

Cuenca-Garcia, Carmen

Cuesta, Eduardo

Cuesta, Víctor Revilla

Cueto, Elías

Cueto-Felgueroso, Luis

Cug, Juraj

Cugy, Emmanuelle

Cui, Bo

Cui, Fangsen

Cui, Qingyu

Cui, Wenyuan

Cui, Xiaoti

Cui, Yi

Cui, Yu

Cui, Zheng

Cuibus, Lucian

Čukić, Milena B.

Cukrov, Neven

Culha, Utku

Culita, Janetta

Culliton, David

Culshaw, Brian

Cummins, Adrian

Cunha, Eunice

Cunningham, Rebecca

Cunningham, Stuart
Cuocolo, Renato

Cuomo, Francesca

Curado, António

Curado, Manuel

Curci, Gabriele

Curcio, Alessandro

Curcio, Mariangela

Curecheriu, Lavinia Petronela

Curiac, Daniel-Ioan

Ćurković, Helena Otmačić

Curra, Edoardo

Curti, Mitrofan

Curti, Rémi

Curtis, Robin

Curto, Adrián

Curto, Domenico

Cusidó, Jordi

Custídio, João

Cutchin, Steve

Cutright, Teresa J.

Cuzman, Oana Adriana

Cvitić, Ivan

Ćwieląg-Piasecka, Irmina

Ćwikła, Grzegorz

Ćwiklak, Janusz

Cycoń, Mariusz

Cyklis, Piotr

Cyprych, Konrad

Cyrus, Václav

Cysewski, Piotr

Cytlak, Urszula

Czaban, Stanislaw

Czamara, Krzysztof

Czapiewska, Agnieszka

Czapik, Przemysław

Czaplewski, Cezary

Czaplewski, Krzysztof

Czarnecki, Lech

Czarnecki, Slawomir A.

Czarnocki, Zbigniew

Czarnota, Joanna

Czech, Katarzyna

Czech, Piotr

Czechowski, Leszek

Czeiter, Endre

Czekaj, Dionizy

Czekała, Wojciech

Czerniawski, Thomas

Czerwińska, Agnieszka

Czerwinska, Areta Magda

Czerwinski, Robert 
Czibula, Gabriela

Czlapka-Matyasik, Magdalena

Czyńska, Klara

Czyż, Zbigniew

D' Acunto, Mario

D'Acierno, Luca

D'Adamo, Idiano

D'Agostino, Carmelo

D'Agostino, Carmine

D'Alfonso, Tiziana

D'Alonzo, Marco

D'Alvia, Livio

D'Amato, Chiara Anna

D'Amato, Egidio

D'Amato, Michele

D'ambrogio, Walter

D'Amore, Matteo

D'Andrea, Antonio

D'Angelo, Gianni

D'angola, Antonio

D'Aniello, Mario

D'Arco, Annalisa

D'Ascenzi, Carlo

D'Auria, Daniela

D'Auria, Francesco

D'Auria, Luca

D'Autilia, Roberto

D'Ercole, Simonetta

D'Hemecourt, Pierre A.

D'Oca, Maria Cristina

D'Orazio, Dario

D'Orazio, Giovanni

D'Oro, Salvatore

D'Souza, Malcolm

D'Ulizia, Arianna

D’Urso, Sebastiano

Da Fonseca, André Ribeiro

Da Lio, Cristina

Da Silva Costa, Marco André

Da Silva, Eric

Da Silva, Jaime Pedro Oliveira

Da Silva, Juarez Bento

Da Silva, Mario Marques

Da Silva, Pedro Raposeiro

Da Vinha G. Da Silva, Maria Manuela

Da, Huo

Dabagov, Sultan B.

Dabija, Ana-Maria

Dabiri, Arman

Dabros, Michal

Dabrowiak, James
Dąbrowska, Agnieszka

Dabrowska, Dominika

Dąbrowska, Jolanta

Dabrowski, Janusz

Dabrowski, Joel

Dąbrowski, Mariusz

Dabrowski, Pawel S.

Daculsi, Guy

Dadelo, Stanislavas

Dadic, Martin

Dadić, Zvonimir

Dadlani, Aresh

Daeyoung Kim, Daeyoung

Daga, Alessandro Paolo

Dagdelen, Turker

Daghbouj, Nabil

Daghio, Matteo

Dagliati, Arianna

Dahiya, Rajiv

Dahle, Sebastian

Dahmane, Mohamed

Dai, Jian

Dai, Jiguo

Dai, Yifan

Dai, Zhaohua

Daim, Tugrul

Daksiya, Velautham

Dal Borgo, Mattia

Dal Palù, Doriana

Dalaklis, Dimitrios

Dale, Erica A.

Dale, Sara

Dalefield, Rosalind

Dalessandri, Domenico

Dalgleish, Mat

Dalla Mora, Tiziano

Dalla Vedova, Matteo D. L.

Dallenga, Tobias

Dalverny, Olivier

Daly, Kristian

Dama, Murali

Damacharla, Praveen

Damanpack, A. R.

Damasevicius, Robertas

DÁmato, Roberto

Dames, Kevin D.

Damigos, Matthew

Damjanović, Darko

Dammaschke, Till

Dan Thien, Nguyen

Dan, Dobrota 
Dana, Stancekova

Danac, Ramona

Danang

Dando, Robin

Danel, Roman

Danese, Giovanni

Daneshazarian, Reza

Daneshgar, Saba

Daneshvar, Hossein

Dang, Ji

Dang, Tri Nguyen

Dang, Vinh

Dani, Samir

Daniel, Bill

Daniel, Christophe

Daniela, Ailincai

Daniela, Linda

Daniellou, Richard

Danihelová, Anna

Danijela, Bogner

Danila, Delia

Danish, Sayed Mir Shah

Danjou, Pierre-Edouard

Danko, George

Danko, Matúš

Dannélls, Dana

Dannenberg, Roger

Danner, Simon

Danner, Tobias

Dannier, Adolfo

Danso-Boateng, Eric

Dantan, Aurélien

Danti, Serena

Danza, Ludovico

Danzi, Enrico

Dao, Nhu-Ngoc

Dao, Quy-Thinh

Dao, Son Duy

Daoud, Bilel

Dapena, Adriana

Darabant, Adrian Sergiu

Daraghmeh, Adnan

Daragó, Adam

Darbeheshti, Neda

Dardano, Principia

Dark, Michael

Darmon, Michel

Daróczy, Bálint

Daroń, Marta

Dartnell, Peter

Darvini, Giovanna
Darwish Ahmad, Adnan

Darwish, Muge Mukaddes

Darwish, Omar

Das, Abhishek

Das, Anirban

Das, Anup

Das, Anushree

Das, Manohar

Das, Pradip

Das, Pratibhamoy

Das, Subha

Das, Subhashis

Das, Suradip

Dascalu, Ana Maria

Dascalu, Traian

Dasgupta, Anurag

Dasgupta, Arundhati

Dashtian, Hassan

Dashtipour, Kia

Dasireddy, Venkata D. B. C.

Dassios, Ioannis

Dasygenis, Minas

Daszykowski, Michał

Datcheva, Maria

Datta, Hia

Datta, Rahul

Datta, Rupsa

Datta, Shubhashis

Dattola, Serena

Daudeville, Laurent

Daugevičius, Mykolas

Daunys, Gintautas

Davalli, Angelo

Davalos-Salas, Mercedes

Davarpanah, Afshin

Davey, Thomas A. D.

David, Andrej

David, Gurney

David, Jean-Pierre

David, Uriarte

David, Vlad Laurentiu

Davidrajuh, Reggie

Davidson, Josh

Davila, Juan Carlos

Davila, Kenny

Davis, James

Davis, Lloyd

Davis, Neil

Davis, Robert

Davison, Adrian K.

Davoudi, Masoume 
Davoudi, Rouzbeh

Davy, John

Davydenko, Kateryna

Dawadi, Babu R.

Dawes, Judith

Day, Steven

Dayal, Kavina

De Aberasturi, Dorleta Jiménez

De Almeida, Ana

De Almeida, José António

De Almeida, Jose Manuel

De Almeida, Maria Catarina

De Andrade Lima, Micael

De Araújo Brazão Farinh

De Arcas, Guillermo

De Bellis, Palmira

De Benedictis, Riccardo

De Beule, Pieter

De Biase, Alberto

De Boer, Maaike H. T.

De Bona, Francesco

De Bonis, Alberto

De Boom, Cedric

De Camargo, Felipe Vannucchi

De Carlo, Filippo

De Caro, Fabrizio

De Caro, Liberato

De Carvalho Jerónimo Barbosa, Inês

De Castro Maqueda,

De Castro, Paulo M. S. T.

De Castro, Rosa M.

De Cózar, Abel

De Crescenzo, Carmen

De Crisci, Antonio

De Croon, Guido

De Dear, Richard

De Diego, Isaac Martín

De Dios, Carlos

De Domenico, Stefania

De Falco, Giuseppe

De Felice, Fabio

De Felipe Mesquida, David

De Ferri, Lavinia

De Figueiredo, Felipe Augusto Pereira

De Freitas Pereira, Tiago

De Gaetani, Carlo Iapige

De Gaspari, Alessandro

De Gijt, Jarit

De Giorgi, Maria Luisa

De Graaf, Ger

De Iacovo, Andrea
De Jesus Oliveira, Victor Adriel

De La Cruz Cardenosa Rubio, Maria

De La Luz Cádiz-Gurrea, María

De La Morena, Joaquin

De La O Serna, José Antonio

De La Piedra, Antonio

De La Rosa Velasco, Ángel

De La Rosa, Ramón

De La Sen, Manuel

De Lacey, Antonio Lopez

De Leo, Alfredo

De León, Alberto Sanz

De Los Santos Guerra, José

a, Catarina Correia De Luca, Gaetano

De Luca, Pierantonio

De Luca, Stefano

De Luna, Andrea

De Macedo, Carina Regina

De Marco, Raffaella

De Marco, Rossella

De Martinis, Massimo

De Martinis, Valerio

De Martino, Andrea

De Martino, Attilio

De Masi, Rosa Francesca

De Matteis, Valeria

De Melo, Marcelo Morais Rodrigues

De Miguel, Ignacio

De Mingo López, Luis Fernando

De Miras, Jérôme

De Moura, Joaquim

De Nadai, Marco

De Napoli, Luigi

De Nardo, Luigi

De Nicola, Antonio

De Ninno, Antonella

De Oliveira Junior, Marcos

De Pace, Francesco

De Pace, Pierangelo

De Paepe, Boel

De Pascale, Francesco

De Pasquale, Claudio

De Regis, Michele

De Richter, Sebastien Kiesgen

De Rubeis, Tullio

De San Antonio-Gómez, Carlos

De Sanctis, Mauro

De Santis, MIchele

De Santos Berbel, César

De Sensi, Daniele

De Simone, Francesco 
De Smedt, Valentijn

De Smet, Timothy S.

De Sousa, Ana Margarida Luís

De Sousa, Bruno Macedo

De Sousa, Jose T.

De Stefani, Alberto

De Stefano, Luca

De Stefano, Rosa

De Troyer, Olga

De Valcárcel, Germán J.

De Vicente, Jesus

De Vietro, Nicoletta

De Vito, Giuseppe

De Vito, Saverio

De Waele, Jo

De Zeeuw-van Dalfsen, Elske

De Zinis, Luca Oscar Redaelli

De Zordi, Nicola

De, Tanima

Deac, Cristian

Deacon, Glen

Deaconu, Adrian

Deaconu, Ioan-Dragos

Deak, Gyorgy

Deal, Brian

Deb Nath, Nipun

DeBacker, Hans

Debaene, Guillaume

Debayle, Johan

Debéda, Hélène

Debeleac, Carmen

Debnath, Madhuri

Debole, Franca

Debono, Carl James

Dębowski, Marcin (Uniwersytet Warminsko-Mazurski)

Dębowski, Marcin (Wrocław University of

Environmental and Life Sciences)

Dębska, Bernardeta

DeBuc, Delia Cabrera

DeCastro, Carlos

Dechouniotis, Dimitris

Decker, Richard

Deckert, Alice

Decostere, Bjorge

Defoe, Jeff

Defraigne, Pascale

Degee, Herve

Degirmenci, Kenan

Degli Esposti, Micaela

Déguernel, Ken
Deharvengt, Stephane

Dehghanghadikolaei, Amir

Dehghani, Hamidreza

Dehghani, Milad

Deigner, Hans Peter

Deilmann, Thorsten

Deiterding, Ralf

Deja, Mariusz

Deka, Lipika

Dekavalla, Maria

Del Águila, Ana

Del Ama Gonzalo, Fernando

Del Carmen Pérez-Fuentes, María

Del Carmen Rodríguez-Hernández, María

Del Carmen Valls Martínez, María

Del Castillo-Santaella, Teresa

Del Cerro Velázquez,

Del Core, Giuseppe

Del Hougne, Philipp

Del Mar Carlos Alberola, Maria

Del Mar Sanchez-Perez, Maria

Del Moral Torres, Fernando

Del Pilar Castro-García, María

Del Pilar Suarez Alonso, Maria

Del Pino, Javier

Del Pizzo, Silvio

Del Real-Romero, Juan Carlos

Del Sol Illana, Irene

Del Val, Jesús

Del Val, Lara

Del Vecchio, Alessandro

Del Vecchio, Carmen

Del Zoppo, Marta

De-la-Cruz-Torres, Blanca

De-la-Fuente, Eusebio

Delaney, Keith B.

Delannay, Francis

Delavar, Mahshid

Delavarpour, Nadia

Delcea, Camelia

Deleanu, Lorena

Delebarre, Christophe

Deleforge, Antoine

DeLellis, Pietro

Delfs, Jens Olaf

Delgado Prieto, Miguel

Delgado Rodrigues, Jose

Delgado Sobrino, Daynier Rolando

Delgado, Jorge 
Delgado-Bonal, Alfonso

Delgado-Sanchez, Jose-Maria

Delichatsios, Michael A.

Deligani, Maryam Afzali

Deligiannakis, Georgios

Deliyanti, Devy

Dell'Accio, Francesco

Dell'Agli, Gianfranco

Dell'Aglio, Marcella.

Dell'Amico, Anna

Dell'Anna, Federico

Dell'Olio, Francesco

Della Malva, Antonella

Della Torre, Augusto

Della Torre, Stefano

Della Vedova, Gianluca

Della Volpe, Claudio

Dellavia, Claudia

Delle Monache, Stefano

Delle Rose, Marco

Del-Rio-Ruiz, Ruben

Delrue, Florian

Deluka-Tibljaš, Aleksandra

Dematteis, Niccolò

Dembele, Sounkalo

Dembińska, Izabela

Dembinski, Roman

Deme, Pragneya

Demers, Hendrix

Demers, Isabelle

Demertzis, Konstantinos

Demetracopoulos, Alexander C.

Demidenko, Eugene

Demidova, Galina L.

Demidova, Liliya

Demir, Baris

Demir, Ebru

Demirci, Ibrahim

Demongeot, Jacques

Demontis, Valeria

Demrozi, Florenc

Demyanov, Vladislav

Dénes, Attila

Deneva, Margarita

Deng, Dongyang

Deng, Lei

Deng, Shihai

Deng, Shuo

Deng, Tong

Deng, Wei

Denić-Jukić, Vesna
Denis, Lise Ann St.

Denis, Vivien

Denisiewicz, Arkadiusz

Deniziak, Stanisław

Denkova-Kostova, Rositsa Stefanova

Dentamaro, Vincenzo

Denti, Enrico

Dentzman, Katherine

Denzumi, Shuhei

Depczynski, Wojciech

Depero, Laura E.

Depiante, Marco

Deppe, Christian

Deptuła, Milena

Der Wiesche, Stefan Aus

Dera, Dimah

Deracinois, Barbara

Dercz, Grzegorz

Derezińska, Anna

Derimow, Nicholas

Deriszadeh, Ali

Derkatch, Svetlana

Derlatka, Anna

Derlukiewicz, Damian

Derry, Paul

Derviskadic, Asja

Desai, Jaydip

Descrovi, Emiliano

Deserno, Thomas M.

Desgranges, Lionel

Deshpande, Anagh

Desmarets, Christophe

Desmet, Jan

Desnitsky, Vasily

Dessent, Caroline

Dessi, Claudia

Dettori, Marco

Dev, Soumyabrata

Devadas, Mary Sajini

Devaney, Nicholas

Devasurendra, Amila

Devdariani, Alexander

Deveci, Muhammet

Devia, Francesco

Devirgiliis, Chiara

Devkota, Jagannath

Dewitte, Olivier

Dey, Debayan

Dey, Kamol

Dey, Somdip

Dey, Vikram 
Deyhle, Hans

Dhakal, Bhubaneswor

Dhakar, Nilesh Kumar

Dhaliwal, Gurjot S.

Dhamdhere, Ajit

Dhanjai, Dhanjai

Dhariwal, Rohit

Dharmarathne, Suranga

Dharmaratne, Priyanga

Dhulipala, Somayajulu

Di Angelo, Luca

Di Bacco, Mario

Di Baldassarre, Angela

Di Bari, Lorenzo

Di Blasio, Gabriele

Di Bona, Gianpaolo

Di Caprio, Francesco

Di Caprio, Giuseppe

Di Carlo, Andrea

Di Cencio, Andrea

Di Ciaccio, Fabiana

Di Dato, Valeria

Di Domenico, Giovanni

Di Federico, Vittorio

Di Filippo, Rocco

Di Fiore, Adolfo

Di Fiore, Luciano

Di Foggia, Giacomo

Di Giuseppe, Dario

Di Ilio, Giovanni

Di Laora, Raffaele

Di Lena, Gabriella

Di Leo, Giuseppe

Di Lernia, Annamaria

Di Lillo, Paolo

Di Luzio, Giovanni

Di Maio, Francesco

Di Maio, Luciano

Di Marco, Alessandro

Di Marco, Piergiuseppe

Di Martino, Antonio

Di Martino, Patrick

Di Matteo, Alberto

Di Matteo, Lucio

Di Mauro, Maria

Di Mauro, Mario

Di Nardo, Fabio

Di Nardo, Francesco

Di Natale, Concetta

Di Noia, Luigi Pio

Di Nunno, Fabio
Di Nunzio, Giorgio Maria

Di Nunzio, Luca

Di Nunzio, Mattia

Di Palma, Pasquale

Di Paola, Rosanna

Di Persio, Luca

Di Pietra, Vincenzo

Di Pietro, Paola

Di Risio, Marcello

Di Ruberto, Cecilia

Di Sarno, Luigi

Di Schino, Andrea

Di Sieno, Laura

Di Sotto, Antonella

Di Vito, Vittorio

Di Zazzo, Erika

Diac, Mădălina Maria

Diaconeasa, Mihai

Diaconeasa, Zorita

Diaconita, Vlad

Diakhate, Malick

Diamantoulakis, Panagiotis D.

Diana, Sabău-Popa Claudia

Diao, Xiumin

Dias Junior, Antonio Gregorio

Dias Pereira, Luisa

Dias, Albino Alves

Dias, Alfredo M. P. G.

Dias, Morgado

Dias, Sofia B.

Diaz Blanco, Manuel Jesus

Díaz De Tuesta, Jose Luis

Díaz Fernández, Belén

Díaz García, Antonio F.

Díaz, Cristina Moreno

Diaz, Inaki

Díaz, Iván Muñoz

Díaz, Jose

Díaz-Cacho Medina, Miguel Ramón

Díaz-De Alba, Margarita

Diaz-Elsayed, Nancy

Díaz-Fuentes, Daniel

Díaz-Ortiz, Ángel

Diaz-Somoano, Mercedes

DiBerardino, Louis

Dichiara, Anthony

Dickinson, Sally

Dictor, Marie-Christine

Diederichsen, Kyle

Diego, Belén Curto

Diego, Corrochano 
Diego, Fortugno

Diella, Valeria

Diémoz, Henri

Dieterlen, Alain

Dietrich, Sebastian

Dietzen, Christiana

Diez, José M.

Díez, Luis Francisco

Diez, Yago

Díez-González, Javier

Díez-Jiménez, Efrén

Diezma, Belen

Digiesi, Salvatore

Diglio, Antonio

Dijk, Thomas Van

Dijksman, Joshua A.

Dijkstra, Albert J.

Dikova, Ts D.

Diller, Tom

Dimanche-Boitrel,

Dimarogona, Maria

Dimas, Athanassios

Dimitra, Sakellari

Dimitrakopoulos,

Dimitrakopoulou-Strauss, Antonia

Dimitri, Rossana

Dimitrios, Bompos

Dimitrios, Chronopoulos

Dimitriou, Vasilis

Dimopoulou, Maria

Dimoulas, Charalampos

Dimri, Manali

Dimyadi, Johannes

Dinache, Andra

DiNardo, Andrew

Dinardo, Francesca

Dinardo, Giuseppe

Dindorf, Ryszard

Dinescu, Gheorghe

Dineva, Adrienn

Ding, Aidong Adam

Ding, Chen

Ding, Chien-Fang

Ding, Jianguo

Ding, Kai

Ding, Lin

Ding, Wei Dan

Ding, Yuan

Ding, Yuanzhao

Ding, Yung-Ching

Ding, Zhao
Ding, Ziyun

Dingler, Tilman

Dinh, Anh

Dinh, Toan

Dini, Luciana

Dini, Pierpaolo

Dinic, Jelena

Dinis, Maria De Lurdes

Dinis, Pedro

Dinis, Rui

Dinis-Oliveira, Ricardo Jorge

Dinita, Alin

Diniz, Carmen

Dinneen, Michael J.

Diogo, Canavarro

Diogo, Patricia

Dioguardi, Mario

Diomede, Francesca

Diomede, Tommaso

Dionísio, Rogério

Dioşan, Laura

Dipnall, Joanna

Diraco, Giovanni

Dirin, Amir

Dissanayaka, Waruna

Ditaranto, Nicoletta

Dittman, Sebastian

Divakaruni, Srinivas

DiVerdi, Joseph

Divić, Vladimir

Dixon, David A.

Dixon, Steve

Diykh, Mohammed

Dizo, Jan

Dizqah, Arash M.

Djafari-rouhani, Bahram

Djajadi, Demi Tristan

Djambazov, Georgi

Djavadian, Shadi

DJEBBARI, Abdelghani

Djebbi, Mohamed Amine

Djedidi, Oussama

Djenouri, Youcef

Djeraba, Chaabane

Djerioui, Ali

Djeziri, Mohand

Djordjevic, Goran Lj.

Djuric, Ana

Djurović, Siniša

Długosz, Olga

Dmitrievskii, Vladimir 
Do Carmo, Sergio J. C.

Do Vale, Francisco

Do, Thuy

Doan, Dat

Dobešová, Zdena

Dobmann, Gerd

Dobo-Nagy, Csaba

Dobosz, Marek

Dobránszky, János

Dobre, Ciprian

Dobre, Mariana

Dobre, Octavia

Dobre, Robert Alexandru

Dobrea, Dan-Marius

Dobrev, Nikolai

Dobrev, Yassen

Dobreva, Antoaneta

Dobrikova, Anelia G.

Dobroka, Mihaly

Dobrotă, Dan

Dobrovolny, Hana M.

Dobrowolski, Andrzej

Dobrzańska, Joanna

Dobrzanski, Leszek Adam

Dobrzanski, Pawel

Dobrzycki, Arkadiusz

Dobrzynski, Piotr

Dobslaw, Daniel

Docimo, Teresa

Dodi, Gianina

Dodson Peterson, Jean Catherine

Dodun, Oana

Doering, John (Jay)

Dofe, Jaya

Dogančić, Dragana

Doganis, Philip

Dogkas, George

Dogossy, Gábor

Dohnal, Fadi

Doi, Kotaro

Doitrand, Aurélien

Dokladalova, Eva

Dokšanović, Tihomir

Dolacek-Alduk, Zlata

Dolatabadi, Nader

Dolatian, Hossep

Dolci, Mathias

Dolega, Boguslaw

Dołęga, Waldemar

Dolenec, Rok

Dolezel, Diane M.
Dolezel, Petr

Dolfing, Jan

Dolgikh, Grigory Ivanovich

Doligalski, Michal

Döllinger, Michael

Dolmatov, Dmitry O.

Dolnik, Bystrik

Dolníková, Erika

Dołowy, Małgorzata

Dolzyk-Szypcio, Katarzyna

Dołżyńska, Magdalena

Domagała, Sławomir

Domalik-Pyzik, Patrycja

Domaneschi, Marco

Domański, Adam

Domanski, Pawel D.

Domaratzki, Michael

Domb, Menachem

Domen, Andreas

Domenico

Domenico, Mariano Di

Domian, Ewa

Domin, Jaroslaw

Domingues, Inês

Domingues, Nuno

Domínguez Álvarez, Enrique

Dominguez Vías, German

Dominguez, Jaime

Dominguez, Jorge

Dominguez, Juan Carlos

Domínguez, María Elena Alemán

Dominguez, Ruben

Dominguez, Sergio

Domínguez-Morales, Juan P.

Domínguez-Morales, Manuel

Domínguez-Navarro, Jose Antonio

Domínguez-Perles, Raúl

Dominguez-Ramos, Antonio

Domínguez-Vías, Germán

Dominik, Janusz

Domljan, Danijela

Domski, Jacek

Don, Aakash Welgamage

Don, Korycansky

Donadello, Simone

Donaire, Manuel

Donaire-Avila, Jesús

Donaj, Gregor

Donaldson, Burl

Donaldson, Ross J.

Donãte, Cristina Martín 
Donati, Lorenzo

Donati, Massimiliano

Doncescu, Andrei

Donelli, Massimo

Dong, Bowei

Dong, Chuan-Zhi

Dong, Dianbiao

Dong, Hua

Dong, Junliang

Dong, Kejun

Dong, Qinglin

Dong, Qiuchen

Dong, Wen

Dong, Xiaobo

Dong, Zheng

Doni Jayavelu, Naresh

Donne, Kelvin

Donner, Reik (Magdeburg-Stendal

University of Applied Sciences)

Donner, Reik (Potsdam Institute for Climate Impact Research)

Donnermeyer, David

Donti, Anastasia

Donti, Olyvia

Doosttalab, Ali

Dopazo, Gonzalo Astray

Dopierała, Łukasz

Dorado, Ruben

Dorafshan, Sattar

Doranehgard, Mohammad Hossein

Dordevic, Dani

Doria, Alberto

Dörling, Bernhard

Dorner-Reisel, Annett

Dorocki, Sławomir

Doron, Ramy

Doronzo, Domenico M.

Doroszko, Michał

Dorotea, Nuno

Doroudiani, Saeed

Doroz, Rafal

Dorożyński, Przemysław

Dörpinghaus, Jens

Dorrah, Ahmed

Dorrío, Benito V.

Dorsey, Kristen L.

Dos Reis, Paulo Nobre Balbis

Dos Santos, João Miguel Marques

Dos Santos, Serge

Dospinescu, Octavian

Dosso, Dennis
Dostatni, Ewa

Doukas, Dimitrios

Doumanis, Ioannis

Doumenis, Gregory

Douvartzides, Savvas L.

Douvas, Antonios

Doval Miñarro, Marta

Dovgal, Igor Vasilyevich

Dovjak, Mateja

Dowell, Earl H.

Downey, Austin

Downing, Charles

Dowon, Bae

Doyle, Daniel

Doyle, Michael R.

Doynikova, Elena

Dozono, Hiroshi

Drab, Mitja

Drabińska, Natalia

Drachal, Krzysztof

Dragan, Dejan

Dragan, Ioan

Dragan, Mirela

Draganić, Hrvoje

Draganov, Ivo

Draganová, Katarína

Drago, Carlo

Dragoi, Elena Niculina

Dragoi, Marian

Drăgoi, Mircea Viorel

Dragoman, Mircea

Dragomir, Alin

Dragomir, Mihai

Dragos, Machidon

Dragovic, Besim

Dragović-Uzelac, Verica

Dranka, Maciej

Drapaca, Corina

Drapikowski, Paweł

Dražić, Ivan

Dreesen, Philippe

Dreglea, Aliona Ivanovna

Dremin, Viktor

Drese, Klaus Stefan

Dresp-Langley, Birgitta

Dressel, Andrew

Dressel, Martin

Drevet, Richard

Drewniak, Józef 
Drexler, Andreas

Drexler, Daniel

Drezewski, Rafal

Drgan, Viktor

Drgas, Szymon

Drgona, Ján

Drgona, Peter

Drikakis, Dimitris

Drimer, Nitai

Drioli, Carlo

Drissi-Habti, Monssef

Dritselis, Chris

Drobiec, Łukasz

Drobná, Helena

Drobot, Radu

Droge, Greg

Drosos, Vasileios

Drossos, Konstantinos

Drouard, Emmanuel

Drozd, Greg T.

Drozd, Jan

Drozd, Radoslaw

Drozda, Paweł

Drozdov, Andrey

Droździel, Paweł

Druesne, Frederic

Druetta, Pablo

Drulis, Henryk

Drusa, Marian

Druszczynska, Magdalena

Druzhinin, Oleg

Drygała, Izabela Joanna

Drzazga, Michał

Drzeżdżon, Joanna

Drzymała, Tomasz

Du Bois, Els

Du, Guodong

$\mathrm{Du}$, Jianhao

Du, Sijun

Du, Xinli

$\mathrm{Du}$, Yegang

Du, Yuncheng

Du, Zhiyan

Duan, Dongliang

Duan, Guangdong

Duan, HF

Duan, Lian

Duan, Molong

Duan, Nan

Duan, Qiang

Duan, Xiyu
Duan, Yuanyuan

Duanis-Assaf, Danielle

Duarte Correia, Sérgio

Duarte Marafona, José

Duarte, António P. C.

Duarte, Juliana Pacheco

Duarte, Lia

Duarte, Noélia

Duarte, Rui Policarpo

Duarte, Vitor Santos

Dub, Martin

Dubaj, Tibor

Dubbioso, Giulio

Dubey, Harishchandra

Dubey, Nileshkumar

Dubey, Shri

Dubey, Venky

Dublyansky, Yuri

Dubovikov, Evgeny

Dubuc, Brennan

Duburs, Gunars

Duchesne, Marc

Ducman, Vilma

Ducournau, Guillaume

Duczek (Eisenträger), Sascha

Duczmal-Czernikiewicz, Agata

Duda, Sławomir

Dudás, László

Dudczyk, Janusz

Dudek, Gabriela

Dudek, Krzysztof

Dudhipala, Narendar

Dudkiewicz, Edyta

Dudojc, Boleslaw

Dudziak, Sławomir

Dudzik, Marek

Duer, Stanisław

Dugonjić Jovančević, Sanja

Duka, Adrian-Vasile

Dukić, Walter

Dulau, Lucian Ioan

Dulikravich, George

Dulina, Luboslav

Dulińska, Joanna Maria

Duliu, Octavian G.

Dulova, Niina

Dulska, Agnieszka

Dulski, Mateusz

Duma, Virgil-Florin

Duman, Gazi Murat

Dumbrava, Anca 
Dumbrava, Stefan

Dumic, Emil

Dumitrescu, Bogdan Andrei

Dumitru, Gabriela

Dumont, Joseph

Dumont, Pierre

Dumont, Serge

Dumor, Koffi

Dumur, Frédéric

Dunai, Larisa

Dunaj, Paweł

Dunbar, Scott

Duncan, Olly

Dundar, Ibrahim

Dunderdale, Gary J.

Dunea, Daniel

Dung, Nguyen Dinh

Dunkel, Alexander

Dunn, Jessilyn

Dunn, Mark

Dunne, Druce

Dunsky, Ayelet

Dunst, Paul

Dunstan, Matthew T.

Duong, Van Tu

Duplaga, Mariusz

Dupláková, Darina

Duplančić Leder, Tea

Duprey, Sonia

Dupuis, Pascal

Duque Domingo, Jaime

Duque, Oscar

Duque-Pérez, Oscar

DuQuesnay, David

Duraisamy, Shriram

Durana, Pavol

Durán-Barroso, Pablo

Durand, Marlène

Durán-Valle, Carlos J.

Durão, Luís Miguel P.

Đurasević, Marko

Durazzo, Alessandra

Đurđević, Dinko

Durdyev, Serdar

Durejko, Tomasz

Đurin, Bojan

Ďuriš, Lukáš

Durkalec-Michalski, Krzysztof

Durmus, Dorukalp

Dürr, Robert

Dušek, Karel
Dusny, Christian

Dutertre, Jean Max

Dutescu, Adriana

Duthie, Rory

Dutil, Yvan

Dutler, Nathan

Dutta, Aniruddha

Dutta, Arindam

Dutta, Atri

Dutta, Ayan

Dutta, Biswanath

Dutta, Kingshuk

Dutta, Saikat

Dutta, Sandip

Dutta, Sanjucta

Dutta, Vibekananda

Dutu, Ligia

Duxbury, Scott W

Duzhin, Fedor

Dvorský, Tomáš

Dworak, Paweł

Dworakowski, Ziemowit

Dworecka-Wójcik, Julita

Dyatko, Nikolay A.

Dybkowski, Mateusz

Dyjakon, Arkadiusz

Dykas, Sławomir

Dyke, Shirley J.

Dymerski, Tomasz

Dyomin, Victor

Dyrcz, Czesław

Dyskin, Arcady

Dzedzickis, Andrius

Dzemyda, Gintautas

Dzhanashia, Kristina

Dziadosz, Agnieszka

Dziechciarz, Józef

Dzięcioł, Justyna

Dzieciol, Monika

Dziedzic, Andrzej

Dziedzic, Krzysztof

Dzielski, John

Dzierżek, Jan

Dziewulska, Daria

Dziki, Dariusz

Dzimira, Stanisław

Dzimitrowicz, Anna

Dzionk, Stefan

Dziula, Przemyslaw

Dziurka, Dorota

Dziurkowska, Ewelina 
Dzwierzynska, Jolanta

E., Yiwen

Earthman, James

Eaton, Christopher M.

Ebada, Sherif

Eberhardsteiner, Lukas

Ebisawa, Satoshi

Ebong, Abasifreke U.

Ebrahimi, Khashayar

Ebrahimi, Mehdi

Ebrahimzadeh, Amin

Echeandía, Juan Santos

Echempati, Raghu

Echtermeyer, Andreas

Ecke, Thorsten Holger

Ecker, Melanie

Ecorchard, Petra

Edeas, Marvin

Edee, Kofi

Eden, Tim

Ederra, Iñigo

Edgar, Andrew

Edler, Dennis

Edward, Wilds

Edwini-Bonsu, Stephen

Eerola, Tuomas

Efremenko, Elena

Egan, Paul F

Egberts, Philip

Egedy, Attila

Egger, Jan

Eggermont, Marjan

Eggers, Tatiana

Egi, Yunus

Egidi, Leonardo

Egner, Halina

Egorkin, Vladimir

Eguchi, Kei

Egwebe, Augustine

Ehde, Per Magnus

Ehrmann, Andrea

Eick, Sigrun

Eid, Mohamad

Eid, Rami

Eifler, Matthias

Eilts, Peter

Eisenhaber, Frank

Eisinas, Anatolijus

Eitzinger, Christian

Ekeberg, Dag

Ekergård, Boel
Ekmekcioglu, Erhan

Ekpeni, Leonard

Ekpo, Sunday

Ekuni, Daisuke

El Ayadi, Amina

El Euch, Hichem

EL KADHI, Nabil

El Khalloufi, Fatima

El Mariachet, Jorge

El Mawas, Nour

El Sachat, Alexandros

Elahi, Hassan

El-Amier, Nesma

Elasha, Faris

Elashry, Ahmed

Elbahhar, Fouzia Boukour

El-Bialy, Tarek

Elbouchikhi, Elhoussin

Elbouz, Marwa

Elduque, Daniel

Elena, Lucchi

Elena, Tiuc Ancuța

El-Esawi, Mohamed A.

Elf, Marie

Elgamoudi, Bassam

Elgendy, Mostafa

El-Gewely, Mohamed Raafat

Elghamrawy, Haidy

Elghobashi-Meinhardt, Nadia

Elhadidi, Basman

Elhag, Sami

El-Hakim, Mohab

Elhattab, Ahmed

Elia, Maurizio

Elias Brás, Ana Rita

Elias, Miguel

Elias, Said

Eliseev, Alexander

Eliseev, Sergey

Eliseev, Vladimir

Elizondo-Martínez, Eduardo-Javier

El-Jawhari, Jehan

Elkaseer, Ahmed

Elken, Jüri

El-Kharouf, Ahmad

Elkhodr, Mahmoud

Ellahi, Rehmat

Ellepola, Kassapa

Eller, Fred

Ellett, Felix

Elliot, Thomas 
Elliott, Brent A.

Ellis, Matthew

Ellison, Paul

Elmadih, Waiel

El-Moghazy, Ahmed

Elmogy, Mohammed

Elmquist, Randolph E.

Eloe, Paul W.

Elolimy, Ahmed A.

Elosegui, Ibon

El-Razouk, Hayssam

El-Safty, Sherif

Elsaid, Khaled

Elsäßer, Wolfgang

ElSayed, Ahmed

Elsayed, Nelly

El-Schich, Zahra

El-Shafee, Omar Osama

Elshaw, Mark

Elsisi, Mahmoud

Elsner, Paul

Elsts, Atis

Eltaeib, Tarik

Eltaggaz, Abdelkrem

Eltarabily, Mohamed

Elustondo, Diego

Elvidge, Sean

Elżbieta, Bielecka

Elzner, Petr

Emad, Muhammad Zaka

Emanuele, Guarriglia

Emery, Ashley

Emes, Matthew

Emilia, Paone

Emilian, Turcanu Florin

Emmanouil

Emmanuel, Lhuillier

Emmer, Adam

Emmerich, Lukas

Emmert, Luke A.

Emodi, Nnaemeka

Emoto, Katsura

Empler, Tommaso

Enalou, Hossein Balaghi

Enderlein, Jörg

Endo, Akira

Endo, Hiroyuki

Endo, Patricia Takako

Enescu, Diana

Engelberth, Abigail

Engler, Anna
Enguita, José María

Enkhsaikhan, Boldsaikhan

Enoki, Koji

Enokido, Tomoya

Enrico, Gherlone Felice

Enrique, Juan M.

Enríquez, Jose Gonzalez

Enzinger, Norbert

Epelde, Francisco

Epelde, Gorka

Epicoco, Italo

Eppelbaum, Lev

Eramo, Vincenzo

Erchiqui, Fouad

Ercole, Francesca

Erdakov, Ivan Nikolaevich

Erdélyi, Ján

Erdem, Emre

Eremeyev, Victor

Eremin, Artem

Erenli, Kai

Erhart, Jiři

Eriksen, René Lynge

Erinle, Kehinde

Erisov, Yaroslav

Erkoyuncu, John A.

Ermakov, Dmitry

Ermolov, Ivan

Ernst Seemann, Stefan

Ernstson, Kord

Erny, Guillaume L.

Erokhin, Vasilii

Erol, Selim

Errandonea, Daniel

Erskine, Samuel Kofi

Ersoz, Elhan S.

Ertas, Atila

Ertaylan, Gokhan

Ertel, Klaus

Ertel, Monica

Erturk, Emre

Escamilla, Alfonso

Eschenlohr, Andrea

Escola, José María

Escolano, David

Escolano, Félix

Escorcia Carranza, Ivonne

Escorihuela, Jorge

Escribano-Garcia, Ruben

Escrivá-Escrivá, Guillermo

Escudero, Carlos 
Escudero, Olga

Esfahani Monfared, Yashar

Eshkabilov, Sulaymon L.

Eskandari, Mona

Eskridge, Thomas C.

Eslami, Hossein

Eslam-Panah, Azar

Esler, Colin

Esmaeeli, Roja

Esmaeili, Amin

Esmaeli, Behzad

Espa, Stefania

Espada, Mário

Espada-Bellido, Estrella

Esparza, M. Eugenia Martín

Espejo-García, Borja

Espinosa Fernandez, Almudena

Espirito-Santo, António

Esposito, Antonio

Esposito, Christian

Esposito, Elisa

Esposito, Flavio

Esposito, Gianluca

Esposito, Luca

Esposito, Maria Teresa

Esposito, Serena

Esser, Kjell B.

Essounbouli, Najib

Esteban, Dolores

Esterline, Albert

Estevadeordal, Jordi

Estêvão, João M. C.

Esteves Da Silva, Joaquim C. G.

Esteves, Bruno

Esteves, Eduardo

Esteves, Ivania

Esteves, Micaela Dinis

Esti, Marco

Estrada-Torres, Bedilia

Esveld, Erik

Etemad-Shahidi, A.

Etich, Julia

Etoz, Sevde

Etsion, Izhak

Eugene, Denisov

Eugenio, Fazio

Euiseok, Hwang

Eva Maria

Evangelisti, Claudio

Evans, Leighton

Evans, Stephen
Even, Markus

Everett, Richard

Evin, Emil

Evlyukhin, Andrey B.

Evola, Gianpiero

Evsutin, Oleg

Ewald, Vincentius

Ewen, James

Ewert, Pawel

Eysel-Gosepath, Katrin

Ezazi, Mohammadamin

Ezekiel, Soundararajan

Ezzat, Ahmed Aziz

Faba, Laura

Fabbiano, Laura

Fabbri, Stefano

Fabbrocino, Francesco

Fabelo, Himar

Fabian, Piotr

Fabian, Ralf

Fabiańska, Monika

Fabiszewska, Agata

Fàbrega, Lourdes

Fabregas, Ernesto

Fabregat, Ramon

Fabrikant, Ilya

Fabris, Nicola

Fabrizio, Mauro

Fábry, Stanislav

Facchetti, Giorgio

Facchiano, Angelo

Facchini, Francesco

Facco, Pierantonio

Fadl, Mohamed Sakr

Faella, Giuseppe

Faezipour, Misagh

Fagan, Edward

Faglia, Rodolfo

Fahlstedt, Madelen

Fahr, Hans Jörg

Faillace, Luigi

Faisal Haider, Mohammad

Faisan, Sylvain

Faita, Francesco

Faitli, Jozsef

Fajfar, Iztok

Fakhari, Amin

Fakin, Darinka

Faktorová, Dagmar

Falaschetti, Laura

Falcão, Carlos 
Falchetto, Augusto Cannone

Falcinelli, Stefano

Falcone, Pasquale Marcello

Falconer, Ian R.

Faleschini, Flora

Faley, Michael

Falk, Kristin

Falkenstein, Michael

Falkinham III, Joseph O.

Falkowska, Ewa

Falkowski, Paweł

Falkowski-Gilski, Przemyslaw

Faller, Hugues

Fallon, James

Fallucchi, Francesca

Falsetti, Anthony

Falsone, Giovanni

Falta, Daniel

Fam, Tkhe Kyong

Famà, Fausto

Famoso, Fabio

Fan, Chengyu

Fan, Jizhou

Fan, Kuang-Chao

Fan, Liang

Fan, Lusheng

Fan, Miao

Fan, Xiaoyuan

Fan, Yin

Fan, Yu-Cheng

Fan, Zhongming

Fan, Zipei

Fancello, Francesco

Fanea-Ivanovici, Mina

Fanelli, Emanuele

Fanfarillo, E.

Fang, Chen

Fang, Chih Yuan

Fang, Guangyang

Fang, Peng

Fang, Runchen

Fang, Shih-Hau

Fang, Yu-Min

Fanghella, Pietro

Fangrat, Jadwiga

Faniayeu, Ihar

Fanning, Seamus

Fantazy, Kamel

Fantetti, Alfredo

Fantozzi, Fabio

Fapojuwo, Abraham
Fara, Laurentiu

Faraci, Carla

Faraci, Francesca Dalia

Farago, Andras

Farago, Paul

Farahani, Ali

Farahani, Behzad V.

Farahnakian, Fahimeh

Faraj, Rami

Farajpour, Ali

Faraone, Immacolata

Faraut, Gregory

Farcas, Anca Corina

Fard, Mohammad

Farè, Silvia

Fareed, Amber

Fareed, Nadeem

Fargnoli, Mario

Farhad, Farnoosh

Farhang, Faezeh

Farhat, Samir

Faria, Paula

Farid, Muhammad Shahid

Farina, Alessandro

Farina, Ilenia

Fariña, Martín Pereira

Farinha, André Tristany

Farinha, José Torres

Farinos, Merle

Farka, Zdeněk

Farkaš, Branimir

Farkoush, Saeid Gholami

Farné, Stefano

Farnell, Damian J. J.

Farooqi, Hafsa

Farouk, Ahmed

Farquhar, Anna

Farrokhifar, Meisam

Farrokhpanah, Amirsaman

Farronato, Giampietro

Farroni, Teresa

Farshbaf, Sepideh

Farshchi Yazdi, Seyed Amir Fouad

Farsiani, Yasaman

Faruqi, Mohammed A.

Farzampour, Alireza

Farzanian, Khashayar

Fasan, Marco

Fascista, Alessio

Faseeh Qureshi, Nawab Muhammad

Fasolino, Ines 
Fassi, Francesco

Fatehi Pouladi, Soheil

Fathi, Masood

Fattah, Kazi Parvez

Fattahi, Jaouhar

Fattorini, Lanfranco

Fattorusso, Luisa

Fauconnier, Dieter

Faus-Matoses, Vicente

Fauzi, Iliana

Favi, Claudio

Favia, Gianfranco

Favre, Julien

Fayaz, Mohammadreza

Fayaz, Muhammad

Fayazfar, Haniyeh

Fayjaloun, Rosemary

Fazakas, Albert

Fazzi, Daniele

Fazzolari, Rocco

Fecarotta, Oreste

Feckan, Michal

Fedel, Mariangela

Fedel, Michele

Fedele, Lorenzo

Fedele, Rosario

Fedorko, Gabriel

Fedorov, Ruslan V.

Fedorov, Vladimir

Fedorowicz, Joanna

Feeney, Andrew

Fei, Tong

Feier, Bogdan

Feijóo, Andrés

Feijs, Loe M. G.

Feinn, Richard S.

Feito, Norberto

Fekete Farkas, Maria

Fekete, Mária Magdolna

Felden, Carsten

Felici-Castell, Santiago

Felipe, José Luis

Feliu, Vicente

Felnhofer, Anna

Feloni, Elissavet G.

Felseghi, Raluca Andreea

Felski, Andrzej

Feltrero, Roberto

Feltynowski, Marcin

Fenerci, Aksel

Feng, Chuang
Feng, Dongming

Feng, Guojin

Feng, Hongbo

Feng, Huicheng

Feng, Kairui

Feng, Lijuan

Feng, Mengling

Feng, Sheng-Wei

Feng, Shilun

Feng, Shuo

Feng, Tao

Feng, Yang

Feng, Yinnian

Feng, Yixuan

Feng, Youcan

Feng, Yunfei

Feng, Zhiyuan

Fenu, Luigi

Fenyvesi, Eva

Fenza, Giuseppe

Fera, Marcello

Ferdani, Daniele

Ferdous, Wahid

Ferenc, Kristály

Ferenc, Tomasz

Ferguson, Frank T.

Ferguson, Neil

Feria Madueño, Adrián

Ferkova, Zelmira

Fernandes Fagundes, Nathalia

Fernandes Lemos Junior, Wilson José

Fernandes Rodrigues, Maria Helena

Fernandes, Cláudia Oliveira

Fernandes, David

Fernandes, Donald

Fernandes, Fábio

Fernandes, João Luís

Fernandes, João Nuno

Fernandes, Mariana Sofia Peixoto

Fernandes, Ricardo M. F.

Fernandes, Tiago A.

Fernández Breis, Jesualdo Tomás

Fernández Cruz, Manuel

Fernández De Las Peñas, César

Fernández Espinar, Teresa

Fernández Fernández, Francisco Javier

Fernández Gámez, Manuel Angel

Fernández Lázaro, Cesar Ignacio

Fernandez Lozano, Jesus

Fernandez Oro, Jesus Manuel

Fernández Uclés, Domingo 
Fernandez Varo, Helena

Fernandez, Alberto

Fernández, Francisco José Vivas

Fernandez, Jose

Fernández, Julio Blanco

Fernández, Mario

Fernández, Óscar Belmonte

Fernandez, Roemi

Fernández-Ahumada, Luis Manuel

Fernandez-Amoros, David

Fernandez-Basso, Carlos

Férnandez-Batanero, Jose M.

Fernández-Calviño, David

Fernández-Campoy, Juan Miguel

Fernández-Canteli, Alfonso

Fernández-Díaz, Elena

Fernández-Fernández, José $\mathrm{M}$.

Fernandez-Fernandez, Raul

Fernandez-Gamiz, Unai

Fernández-García, José Carlos

Fernandez-Granero, Miguel Angel

Fernández-Hernández, Jesús

Fernández-Isabel, Alberto

Fernández-Lázaro, Diego

Fernandez-Lozano, Carlos

Fernández-Martínez, Elena

Fernandez-Martinez, Lorena

Fernandez-Medina, Eduardo

Fernández-Pacheco, Daniel G.

Fernández-Sánchez, Laura

Fernandez-Veiga, Manuel

Fernández-Vigo, José Ignacio

Fernández-Yáñez, Pablo

Fernando, Niroshinie

Ferracuti, Francesco

Ferradosa, Tiago

Ferrández, Daniel

Ferrante, Camilla

Ferrante, Carino

Ferrantelli, Andrea

Ferrara, Liberato

Ferrara, Manuela

Ferrara, Maria Antonietta

Ferrara, Pietro

Ferrari, Claudio (Istituto dei Materiali per

l'Elettronica ed il Magnetismo)

Ferrari, Claudio (University of Florence)

Ferrari, Marco

Ferrari, Paolo

Ferrari, Simone

Ferrarini, Giovanni
Ferraro, Elena

Ferraro, Pietro

Ferras, Carlos

Ferreira Machado, José Manuel

Ferreira, Ana C. M.

Ferreira, Artur

Ferreira, Débora

Ferreira, Fernando

Ferreira, João Canas

Ferreira, Joao Carlos

Ferreira, Joaquim

Ferreira, Johannes

Ferreira, Luís Andrade

Ferreira, Manuel Marques

Ferreira, Marta S.

Ferreira, Paulo Victor R.

Ferreira, Sandra

Ferreira, Sara

Ferreira, Vitor

Ferreiro-González, Marta

Ferrer Peña, Raúl

Ferrera, Maxime

Ferrer-Espada, Raquel

Ferrero Martín, Francisco J.

Ferretti, Elena

Ferretti, Ivan

Ferretti, Luca

Ferri, Luca

Ferro, Giulio

Ferrucci, Massimiliano

Ferruz, Joaquin

Ferster, Colin Jay

Fertsch, Marek

Ferworn, Alexander

Feschet, Fabien

Fesl, Jan

Fetecau, Catalin

Feucherolles, Maureen

Feuchtner, Tiare

Fey, Tobias

Feyissa, Aberham Hailu

Fiala, Pavel

Fialko, Yuri

Fialova, Jitka

Fiandrotti, Attilio

Ficco, Massimo

Ficek, Krzysztof

Fidalgo, Alexandra

Fidan, Ismail

Fidani, Cristiano 
Fidanova, Stefka

Fidel, Salas Vicente

Fidelibus, Corrado

Fidge, Colin

Fidlerova, Helena

Fiedler, Markus

Fiedot-Toboła, Marta

Fiehler, Katja

Figlus, Tomasz

Figueira, Álvaro

Figueira, Flavio

Figueira, Rita

Figueiral, Maria Helena

Figueiredo, Carlos

Figueiredo, Janine

Figueiredo, Pedro

Figueroa, Karina

Figueroa, Maria Josefina

Figuli, Lucia

Fike, Matej

Fiket, Željka

Fikus, Bartosz

Filanovsky, Igor

Filatovas, Ernestas

Filgueiras, Ana Virginia

Filice, Marco

Filice, Simona

Filina-Dawidowicz, Ludmiła

Filip, Ioan

Filip, Peter

Filip, Petr

Filipa Silva, Ana

Filipe, Vitor

Filipescu, Adrian

Filipescu, Mihaela

Filipiak, Michał

Filipkowska, Urszula

Filipova, Inese

Filipovic, Lado

Filippatos, Angelos

Filippini, Mauro

Filippos, Farmakis

Filippov, Andrey

Filko, Damir

Filo, Grzegorz

Finamore, Weiler A.

Finesso, Roberto

Fingas, Merv

Finimundy, Tiane C.

Fink, Dieter

Fink, Wolfgang
Finšgar, Matjaž

Fiok, Krzysztof

Fioravanti, Ambra

Fiore, Mariantonietta

Fiorentino, Gabriele

Fiorentino, Maria Chiara

Fiorentino, Michele

Fiorenza, Roberto

Fioretti, Alessandra

Fiori, Giorgia

Fiorillo, Edoardo

Fiorillo, Luca

Fiorineschi, Lorenzo

Fiorino, Luigi

Fiorito, Francesco

Fiorucci, Marco

Firat, Murat

Firețeanu, Virgiliu S.

Firlag, Szymon

Fisch, Michael

Fischer, Andreas

Fischer, Claas

Fischer, Cyril

Fischer, Daniel

Fischer, Inga Anita

Fiser, Rastko

Fish, Andrew

Fisher, Robert

Fister, Dušan

Fister, Iztok

Fitzgerald, Breiffni

Fitzgerald, Colm

Fitzgerald, Neil

Fitzgerald, Paul C.

Fiumara, Giacomo

Fivel, Marc

Fizzah-Jilani, Syeda

Flagg, David D.

Flamini, Fulvio

Flandin, Lionel

Flater, Erin E.

Flegner, Patrik

Fleischer, Jürgen

Fleming, Charles

Fleming, Cody

Flemmer, Claire

Flemming, Danny

Fletcher, Steven

Flieger, Jolanta

Floch, Jean Marie

Florea, Adrian 
Florea, Bogdan Cristian

Florea, Corneliu

Florencias Oliveros, Olivia

Flores Arias, Jose Maria

Flores, Marta Roig

Flores-Abad, Angel

Florescu, Adriana

Florescu, Margareta Stela

Floria, Pablo

Florian Baron, Camilo

Florimond, Guéniat

Floris, Mario

Floros, George

Florowska, Anna

Flotyński, Jakub

Flower, Rebecca

Fnadi, Mohamed

Fochesatto, Gilberto J.

Fodorean, Daniel

Fogacci, Federica

Foit, Krzysztof

Fomin, Alexey

Fondelli, Tommaso

Fonseca, Elsa S. R.

Fonseca, Felícia

Fonseca, José

Fontana, Federico

Fontana, Juan

Fontanals, Núria

Fontanella, Chiara Giulia

Fontanesi, Claudio

Fonte, Pedro

Fopah-Lele, Armand

Foraboschi, Paolo

FOrbes, Kayn

Forcellini, Davide

Ford, Nicole

Foreman, Matthew

Forget, Aurelien

Forget, Pascal

Foris, Vasile

Forján, Rubén

Forliano, Canio

Formaggioni, Paolo

Formanek, Matus

Formanowicz, Piotr

Formela, Krzysztof

Forment-Aliaga, Alicia

Formenti, Damiano

Formenton, Gianni

Formicola, Valerio
Formisano, Alessandro

Formisano, Antonio

Fornari, Fabrizio

Fornaro, Claudio

Forner, Leopoldo

Forsman, Mikael

Förster, Klaus-Tycho

Fořt, Jan

Forte, Giovanni

Fortenberry, Ryan

Fortes, Sergio

Forteschi, Mauro

Fortier, Marie-Odile

Fortuna, Luigi

Fortunato, Leonzio

Fortuny Santos, Jordi

Fosalau, Cristian

Fosci, Paolo

Foteinos, Michael I.

Foti, Dora

Foucher, Philippe

Foucher, Samuel

Foudeh, Husam A.

Fouladgar, Nazanin

Foumani, Mehdi

Founti, Maria

Fountoulakis, Ilias

Fourlas, George K.

Fowler, David

Fox, Richard

Foysal, Md Javed

Foysi, Holger

Főző, Ladislav

Fracchia, Elisa

Frąckiewicz, Mariusz

Frąckiewicz, Piotr

Fraddosio, Aguinaldo

Fraga, Hugo

Fraga-Corral, Maria

Fragassa, Cristiano

Fragiadakis, Michalis

Fragliasso, Francesca

Fragos, Vassilios

Fraguas-Sánchez, Ana Isabel

Fraile, Alberto

Framenau, Volker

Francés, Víctor Manuel Soto

Francesca Carfora, Maria

Francesca, Roscini

Franceschini, Giordano

Francesco, Cavenago 
Francesconi, Enrico

Francés-Villora, José Vicente

Franch-Parella, Jordi

Francia, Daniela

Frančić, Vlado

Francik, Slawomir

Francis, Adel

Francis, Ashwanth Christopher

Francis, Nidhish

Francisco Javier

Franco, Antonio

Franco, Walter

Franco-Duarte, Ricardo

Franek, Friedrich

Franger, Sylvain

Frank, Arthur

Frank, Irmgard

Frank, Matthias

Frank, Regine

Frankcombe, Terry

Franke, Knut

Franke, Ulrik

Frankewycz, Borys

Frankiewicz, Mariusz

Frankovska, Jana

Frankovsky, Peter

Frański, Rafał

Frant, Maciej

Fränti, Pasi

Franus, Wojciech

Franz, Yohann

Franzoni, Valentina

Fras, Teresa

Frassetto, Fabio

Fraternali, Fernando

Fratu, Octavian

Frazão, Luís

Frazzica, Andrea

Fredianelli, Luca

Freeman, Brian

Freire-Lista, David

Freitas, Manuel José

Frejlichowski, Dariusz

French, Peter

Frenger, Monika

Freni, Gabriele

Fresco-Cala, Beatriz María

Fresnadillo, David

Fresner, Johannes

Freudenberger, Jürgen

Frey, Sandor
Freymueller, Jeff

Frič, Jan

Frid, Vladimir

Frieboes, Hermann

Fried, Miklos

Friedenberg, Jay

Friedlaender, Alex

Friedrich, Christoph M.

Friedrich, Daniel

Friedrich, Rainer

Friedt, Jean-Michel

Frielinghaus, Henrich

Friess, Wilhelm Alexander

Frigau, Luca

Frigura-Iliasa, Flaviu Mihai

Frippiat, Jean-Pol

Frisenda, Riccardo

Friso, Dario

Fritsch, Dieter

Fritz, Torsten

Frivaldský, Michal

Frnda, Jaroslav

Frodeson, Stefan

Froelich, Matthias F.

Fröhlich, Eleonore

Fröjdh, Erik

Frolova, Tatiana S.

Fronczyk, Joanna

Frongillo, Marcello

Froning, Dieter

Frontera, Patrizia

Frontoni, Emanuele

Froriep, Ulrich P.

Fruggiero, Fabio

Frustaci, Fabio

Fryazinov, Oleg

Fryc, Irena

Fthenakis, George

$\mathrm{Fu}$, Chao

$\mathrm{Fu}$, Chenguang

$\mathrm{Fu}$, Chiung-Shiuan

$\mathrm{Fu}$, Hailing

Fu, Qianyi

Fu, Sheng

FU, Tak-chung

$\mathrm{Fu}$, Xianqiang

$\mathrm{Fu}$, Xinyu

Fu, Yangya

$\mathrm{Fu}$, Yucheng

$\mathrm{Fu}$, Yuguang

Fuad, Marwan 
Fuchi, Kazuko

Fuchs-Kittowski, Frank

Fucikova, Anna

Fue, Kadeghe

Fuentes Dominguez, Rafael

Fuentes, Alvaro

Fuentes-Blasco, Maria

Fuentes-Pardo, José María

Fuentes-Pérez, Juan Francisco

Fuerst, Axel

Fuertes-Miquel, Vicente S.

Fugaru, Viorel

Fuhrmann, Arnulph

Fujdiak, Radek

Fujibe, Fumiaki

Fujieda, Naoki

Fujii, Garuda

Fujii, Keisuke

Fujii, Satoshi

Fujimaki, Haruyuki

Fujimoto, Daisuke

Fujimura, Atsushi

Fujimura, Shintaro

Fujisawa, Masanori

Fujita, Goro

Fujita, Kohei

Fujiu, Makoto

Fujiwara, Akihiko

Fukuda, Atsushi

Fukumoto, Fumiyo

Fukushima, Norishige

Fukuta, Naoki

Fukuta, Satoshi

Fulignati, Sara

Fülöp, Ludovic

Fulvio, Re Cecconi

Fulzele, Sadanand

Funari, Marco Francesco

Funari, Riccardo

Funato, Noriko

Fung, Rong-Fong

Fung, Wai-keung

Funken, Johannes

Furche, Johannes

Füredi, András

Furko, Monika

Furlan, Kaline P.

Furlani Bastos, Alvaro

Furlani, Maurizio

Furler, Robert L.

Furmann, Boguslaw
Furse, Cynthia

Furtado, André

Furtado, Kalli

Furtak, Janusz

Furtak, Karolina

Furui, Sadataka

Furukawa, Kenji

Furuta, Saori

Furuta, Takuya

Furutani, Ryoshu

Fuscaldo, Walter

Fusco, Adele

Fuso, Francesco

Fuss, Franz Konstantin

Futa, Barbara

Fydrych, Dariusz

Gaal, Mate

Gabaldón, Antonio

Gaballah, Ahmed

Gabbar, Hossam A.

Gabbia, Daniela

Gabl, Roman

Gabor, Paul

Gabrić, Dragana

Gabriel, Maximilian

Gabriel, Velu

Gabriele, Bartolo

Gabriele, Morena

Gabrielli, Alessandro

Gabrielli, Leonardo

Gabrielli, Simona

Gabryś, Katarzyna

Gabrys, Tomasz

Gac, Wojciech

Gachet Paez, Diego

Gackowski, Mariusz

Gadd, Matthew

Gade, Rikke

Gadea-Gironés, Rafael

Gadedjisso-Tossou, Agossou

Gądek-Moszczak, Aneta

Gadipelli, Srinivas

Gadsden, Andrew

Gaffet, Eric

Gafurov, Artur

Gafurov, Marat

Gagaoua, Mohammed

Gagliano, Antonio

Gagliano, Salvina

Gagliardi, Paolo

Gagliardi, Paolo Armando 
Gagneja, Kanwalinderjit

Gago, Eulalia Jadraque

Gaida, Nico Alexander

Gaihre, Bipin

Găină, Luiza Ioana

Gaizauskas, Eugenijus

Gajdošová, Katarína

Gajewska, Danuta

Gajewski, Andrzej

Gajewski, Jan

Gajewski, Marcin

Gajjar, Chirag

Gajowniczek, Krzysztof

Gajpal, Yuvraj

Gajtanska, Milada

Gal, Emese

Gal, Frédérick

GÁLA, Michal

Galachyants, Agnia D.

Galamboš, Michal

Galán De Mera, Antonio

Galán, José Manuel

Galanakis, Iosif

Galán-Díaz, Juan Jose

Galanis, Athanasios T.

Galanis, Nikolaos

Galao, Oscar

Galas, Roman

Galati, Manuela

Galatus, Ramona

Galazka, Miroslaw

Gałązkowski, Robert

Galbiati, Marta

Galea, Christian

Galeano, Diego

Galego, Ludovina

Galehdar, Amir

Galenda, Alessandro

Galgalikar, Rohan

Galhano, Alexandra

Galić, Mario

Galindo, Miguel

Galindo, Ruben

Galindo-Torres, Sergio A.

Galińska, Barbara

Galkiewicz, Jaroslaw

Galla, Stanislaw

Gallardo-Antolín, Ascensión

Gallego, Antolino

Gallego, Jose Rodriguez

Gallego-Durán, Francisco J.
Galli, Federico

Galli, Loris

Galli, Viola

Gallo Cordova, Alvaro

Gallo, Mariano

Gallo, Salvatore

Gallo, Simone

Gallon, Laurent

Galloni, Pierluca

Galluzzi, Renato

Galman, Iwona

Galmes, Sebastia

Galstyan, Vardan

Galuppini, Giacomo

Galushkin, Alexander A.

Galvan Diez, Eduardo

Galvão, João Rafael

Galve, Jorge Pedro

Galvosas, Petrik

Galyaev, Andrey

Gamal, Donia

Gambarini, Gianluca

Gambarova, Pietro G.

Gambaruto, Alberto

Gambino, Alessio

Gambino, Michela

Gamboa, Carolina Bermudo

Gamboa, Hugo

Gambuti, Angelita

Gamec, Ján

Gamez Montero, Pedro Javier

Gammons, Christopher H.

Gamnitzer, Peter

Gamo, Javier

Gamon, Wojciech

Gan, Diane

Gan, Guojun

Gan, Woon-Seng

Gan, Yong X.

Gan, $\mathrm{Yu}$

Ganah, Abdulkadir

Ganapathi Shanmugam, Shankar

Ganczarski, Artur Władysław

Gand, Martin

Gandelli, Emanuele

Gandhi, Om P.

Gandia Franco, Jose Luis

Gandini, Paola

Gandla, Madhavi Latha

Gandolfi, Alberto

Gandzha, Segey 
Ganea, Daniel

Ganesan, Sukirth M

Ganetsos, Theodore

Ganguli, Swetava

Gangwani, Pooja

Gangwar, Kapil

Ganikhanov, Feruz

Gansterer, Margaretha

Ganta, Deepak

Gantenbein, Benjamin

Gantner, Ranko

Ganzfried, Sam

Gao, Bei

Gao, Bin

Gao, Chao

Gao, Cong

Gao, Fei (China)

Gao, Fei (USA)

Gao, Jian

Gao, Jie

Gao, Jiuchun

Gao, Jun

Gao, Mingyuan

Gao, Peichao

Gao, Shang

Gao, Shangce

Gao, Sherry Shiqian

Gao, Shiqiang

Gao, Xiaolong

Gao, Xin

Gao, Xinghua

Gao, Xu

Gao, Yanfei

Gao, Zhe

Gao, Zhiwei

Garapati, Nagasree

Garavaglia, Elsa

Garay-Vitoria, Nestor

Garbovskiy, Yuriy

Garbowska, Monika

Garbowski, Tomasz

Garcez, Estela Oliari

García Botella, Angel

Garcia Cascales, Maria Socorro

García Costa, Alicia Loreto

García Diez, Ana

García Gómez, Sergio

García Gutiérrez, Alberto Eloy

García Kerdan, Iván

García López, Salvador

García Sánchez, Manuel
García Suárez, Víctor Manuel

Garcia, Alfredo

Garcia, Andres Sanz

Garcia, Carlos Martinez

García, Carmelo R.

Garcia, Coralia V.

Garcia, Cristobal

García, David Marín

Garcia, João

Garcia, Jose A.

Garcia, Jose Luis

García, José Miguel

García, Julian Pérez

García, Manuel Pérez

García, Marcelo

García, Marcos

García, Miguel Torres

Garcia, Paola

Garcia, Paulo

Garcia, Pedro

Garcia, Ricardo

García, Roberto

Garcia, Rosario

García, Xavier Gironés

García-Afonso, Óscar

Garcia-Alias, Guillermo

García-Arranz, Mariano

García-Barba, Javier

García-Carrillo, Nuria

García-Ceberino, Juan M.

García-Cerrada, Aurelio

García-Collado, Alberto J.

García-Cuesta, Esteban

García-Delgado, Carlos

García-Díaz, Pilar

García-Fogeda, Pablo

García-García, Carlos

Garcia-Garcia, Leonardo

García-Gil, Diego

García-González, Mercedes

García-Hernández, César

Garcia-Lopez, Eva

García-Magariño, Iván

García-Martín, Juan-Pablo

Garcia-Martin, Raul

Garcia-Mateo, Carlos

García-Mateos, Ginés

García-Moreno, Olga

Garcia-Nevado, Elena

García-Ordás, María Teresa

Garcia-Pardo, Javier 
Garcia-Perez, Arturo

García-Pineda, Miguel

García-Pola, María José

Garcia-Pomar, Juan Luis

Garcia-Porta, Nery

García-Prada, Juan Carlos

García-Redondo, Alberto

García-Ros, Gonzalo

Garcia-Rubio, Rocio

Garcia-Salguero, Mercedes

García-Sanz, Verónica

García-Seoane, Rita

García-Soidán, Jose L.

García-Unanue, Jorge

García-Vázquez, Carlos Andrés

García-Viguera, Cristina

García-Villalón, Angel Luis

Garcia-Yeguas, Ara Celi

Garcovich, Daniele

Gardecki, Arkadiusz

Gardeniers, Han

Gardiner, Philip

Gardziejczyk, Wladyslaw

Garg, Harish

Gargallo-Albiol, Jordi

Gargiulo, Gaetano D.

Gargiulo, Valentina

Gariazzo, Claudio

Garidkhuu, Ariuntuul

Garmire, Elsa

Garmyn, Andrea

Garncarek, Zbigniew

Garneli, Varvara

Garner, Angie

Garner, Tom

Garnero, Gabriele

Garofalo, Carmela

Garofolini, Alessandro

Garoli, Denis

Garramiola, Fernando

Garrido Picazo, María Piedad

Garrido, Juan

Garrido, Lara Febrero

Garroni, Sebastiano

Garrote Revilla, Julio

Garsztka, Przemysław

Gartia, Manas Ranjan

Garvey, Michael

Gary, Kevin

Garza, Paolo

Garzillo, Elpidio Maria
Garzón Ramos, David

Garzon, Eduardo

Gascho, Dominic

Gasco, Gabriel

Gash, Hugh

Gašinec, Juraj

Gąska, Adam

Gáspár, László

Gáspár, Péter

Gasparatos, Dionisios

Gasparetto, Alessandro

Gaspari, Jacopo

Gasparik, Jozef

Gasparski, Alexander

Gassin, Pierre-Marie

Gastaldi, Laura

Gastaldo, Paolo

Gasteratos, Antonios

Gastineau, Romain

Gato, Eric

Gatti, Filippo

Gattolin, Stefano

Gatzen, Caren

Gaubert, Alexandra

Gauch, Susan

Gauden, Piotr

Gaukel, Volker

Gaur, Nishtha

Gaurina-Međimurec, Nediljka

Gautam, Deepak

Gautam, Rekha

Gautam, Siddharth S.

Gavaises, Manolis

Gavazzi, Bruno

Gavina, Alexandra

Gavish, Nirit

Gavrilova, Natalia

Gawalek, Jolanta

Gawęcki, Maciej

Gaweda, Adam E.

Gawel, Kinga

Gawlińska, Katarzyna

Gawronek, Pelagia

Gawronska, Elzbieta

Gawrylczyk, Konstanty Marek

Gawryszewska, Beata Joanna

Gayathri, R. G.

Gazda, Piotr

Gaziv, Guy

Gazizov, Almir

Gazquez Parra, Jose Antonio 
Gazzano, Massimo

Gdawiec, Krzysztof

Ge, Cui

Ge, Dongdong

Ge, Dongjiao

Ge, Ting

Gea, Miguel

Geana, Elisabeta-Irina

Gębara, Piotr

Geberemariam, Thewodros K.

Gebremariam, Kidane Fanta

Géczy, Attila

Gedikli, Ersegun Deniz

Gediminas, Vaičiūnas

Geelan, David

Geem, Zong Woo

Gegenhuber, Nina

Gęgotek, Agnieszka

Geiser, Martial H.

Geitner, Robert

Geladi, Paul

Gelhar, Lynn

Geli, Hatim

Gelissen, E. J.

Gelli, Samuele

Geman, Oana

Gembalczyk, Grzegorz

Geminiani, Alice

Gendaszewska-Darmach, Edyta

Gendek, Arkadiusz

Genedy, Moneeb

Generowicz, Agnieszka

Geng, Ruili

Genge, Bela

Genovese, Andrea

Genovese, Carlo

Genovese, Francesco

Gentile, Carmelo

Gentile, Gennaro

Gentile, Pietro

Gentili, Pier

Gentilucci, Matteo

Gentleman, Eileen

Georgantzinos, Stelios K.

George, Deepu

George, Graeme

George, Nathan

George, Sony

George, Thomas F.

Georgescu, Michael

Georgescu, Mircea
Georgiadis, Teodoro

Georgian, Badicu

Georgiev, Danko D.

Georgiev, Georgi As.

Georgiou, Emmanuel P.

Georgiou, Ioannis T.

Georgoulas, Anastasios

Georgousis, Georgios

Geradts, Zeno

Geraldes, Carlos

Gerard, Celine

Gerasim, Krivovichev

Gerasimov, Vladislav V.

Gerber, Florian

Gerbino, Giovanni

Gerbino, Salvatore

Gere, Attila

Gereke, Thomas

Gerhard, Christoph

Gericota, Manuel

Gerighausen, Heike

Gerislioglu, Burak

Gerke, Jörg

Gerke, Oke

Gerken, Alison R.

Gerloff, Dennis

Germanese, Danila

Germanotta, Marco

German-Sallo, Zoltan

Geronimo, Andrew M.

Gerothanassis, Ioannis P.

Gersen, Henkjan

Gervasi, Osvaldo

Geso, Moshi

Gesuele, Felice

Geszke-Moritz, Małgorzata

Gevirtz, Richard

Gevorkov, Levon

Geyer, Philipp

Ghaani, Mohammad Reza

Ghabchi, Rouzbeh

Ghaderpour, Ebrahim

Ghaffar, Adnan

Ghaffarian, Saman

Ghafurian, Moojan

Ghahari, Seyed Ali

Ghalesari, Abbasali Taghavi

Ghanaati, Shahram

Ghanbari, Abbas

Ghanbarian, Behzad

Ghanbarzadeh-Dagheyan, Ashkan 
Ghandi, Khashayar

Ghanekar, Alok

Ghanem, Marwan

Ghani Zadeh, Hossein

Ghani, Arfan

Ghanmi, Helmi

Ghannay, Sahar

Ghanotakis, Demetrios F

Ghareh Baghi, Arash

Gharib, Mohamad

Gharib, Nima

Ghasemi, Navid

Ghavami, Nooshin

Ghavidel, Ali

Ghayoor, Milad

Ghazijahani, Tohid Ghanbari

Ghazizadeh, Eghbal

Ghemari, Zine

Gheni, Ahmed A.

Gheorghe, Grigoras

Gheorghiu, Andrei

Gheorghiu, Eugen

Gherardi, Francesca

Gherardini, Francesco

Gherman, Bogdan

Gherri, Barbara

Ghiasi, Seyed Mojtaba

Ghidelli, Matteo

Ghinassi, Barbara

Ghinet, Alina

Ghione, Giovanni

Ghisi, Aldo

Ghita, Simona

Ghizzo, Alain

Ghobadi, Mohsen

Ghofrani, Faeze

Gholami, Fatemeh

Gholamipour-Shirazi, Azarmidokht

Ghomian, Taher

Ghonaim, Hassan

Ghorayeb, Sleiman R.

Ghorbanzadeh, Omid

Ghoreishi, Seyede Fatemeh

Ghoreyshi, Mehdi

Ghoreyshi, Seyed Mohammad

Ghosal, Sambuddha

Ghosh, Anindya

Ghosh, Arijit

Ghosh, Arnab

Ghosh, Monoj

Ghosh, Sayan
Ghosh, Subhajit

Ghosh, Subir

Ghosh, Sujoy

Ghosh, Susanta

Ghosh, Swarnava

Ghouse, Shaaz

Ghysels, Mélanie

Giacaman, Rodrigo A.

Giaccu, Gian Felice

Giachetti, Andrea

Giacomelli, Michael

Giacomello, Giampiero

Giacometti, Andrea

Giacomini, Mauro

Giacomino, Agnese

Giakos, George

Giakoumidis, Ilias

Giammatteo, Paolo

Giampaolo, Valeria

Giancola, Emanuela

Gianella, Michele

Gianfranco, Fenu

Gianico, Andrea

Gianluca, Alaimo

Giannakas, Filippos

Giannakeas, Nikolaos

Giannakis, Konstantinos

Giannakopoulos, Konstantinos

Giannaros, Theodore M.

Giannella, Venanzio

Giannì, Aldo Bruno

Giannopoulos, Georgios I.

Giannoulis, Giannis

Giaourakis, Dimitrios G.

Giardina, Mariarosa

Giarola, Valentino

Giasin, Khaled

Gibbs, John

Gibiino, Gian Piero

Gibot, Pierre

Gibson, Christopher

Gibson, Jerry D.

Gibson, Richard

Gibson, Ryan

Giddon, Donald B.

Gidon, Dogan

Giel, Robert

Gienko, Gennady

Giergiel, Mariusz

Gierlak, Piotr

Gierszewska, Magdalena 
Giese, Gill

Giesko, Tomasz

Giglio, Marilena

Gigliotti, Rosario

Gijón-Nogueron, Gabriel

Gil Del Val, Alain

Gil, Ana Belén

Gil, Arturo Aparicio

GIl, Bartosz

Gil, Ignacio

Gil, Javier

Gil, Jorge Juan

Gil, M. L. A.

Gil, Nuno

Gil, Paweł

Gilbert, Mark

Gil-Garcia, Isabel Cristina

Gili, Valerio Flavio

Gilioli, Andrea

Gilja, Gordon

Giljarhus, Knut Erik Teigen

Gill, Muhammad Bilal

Gill, Richard D.

Giller, Katrin

Gillespie, R. Brent

Gillich, Gilbert-Rainer

Gillot, Frédéric

Gilly, Katja

Gil-Martín, Emilio

Gim, Jangwon

Gimenez, Antonio

Gimeno Blanes, Francisco Javier

Gimžauskaitè, Dovilè

Gins, Wouter

Ginszt, Michał

Gioia, Ciro

Gioia, Dario

Giomelakis, Dimitrios

Gionata, Salvietti

Giordan, Enrico

Giordano, Nicolo

Giordano, Stefano

Giorgetti, Andrea

Giorgia, Ghiara

Giorgio, Gosti

Giorgio, Ivan

Giorgio-Serchi, Francesco

Giorio, Chiara

Giosio, Dean

Giosuè, Chiara

Giotopoulos, Konstantinos
Giotto, Francine M.

Giovagnoli, Raffaela

Giovannelli, Pia

Giovanni, Fabbrocino

Giovenale, Emilio

Giraldo, Mario

Giraldo-Londoño, Oliver

Girão Silva, Rita

Girard, Pierre-Marie

Giraud, Marie Noëlle

Giray, Tugrul

Girbacia, Florin

Girbés-Juan, Vicent

Girelli, Chiara Roberta

Giri, Paritosh

Girjob, Claudia

Girolami, Marco

Giron Borrero, Sara

Girovský, Peter

Gisbert-Garzarán, Miguel

Gittler, Thomas

Giudice, Amerigo

Giudice, Fabio

Giudice, Giuseppe Lo

Giudice, Oliver

Giudicepietro, Flora

Giudicianni, Carlo

Giuffrida, Maria

Giuffrida, Nadia

Giuliano, Romeo

Giulotto, Enrico

Giunta, Marinella

Giuranno, Donatella

Giurg, Mirosław

Giuseppe, Varvara

Giuseppina, Basini

Giusto, Daniele

Giza, Andrzej

Gizzi, Alessio

Gizzi, Fabrizio Terenzio

Gjeldum, Nikola

Gjevestad, Jon Glenn

Gjörloff Wingren, Anette

Gkantou, Michaela

Gkatzia, Dimitra

Gkonis, Panagiotis K.

Gkountakou, Fani

Głąbowski, Mariusz

Głąbska, Dominika

Gladysz, Bartlomiej

Glaner, Marcus 
Glascock, Matthew S.

Glass, Samuel V.

Glasser, Fredrik

Glasser, Leslie

Glassley, William Edward

Glavas, Hrvoje

Glavatskiy, Kirill

Glazar, Irena

Głębocki, Robert

Glew, David

Glibowski, Paweł

Glicksman, Leon

Gligor, Delia Maria

Glinicki, Michał A

Głomb, Przemysław

Glonek, Grzegorz

Glovnea, Romeo

Głowacka, Aleksandra

Głowacki, Paweł

Glowacz, Adam

Glowinska, Ewa

Głowiński, Sebastian

Głuch, Jerzy

Głuchowski, Andrzej

Gluck, Jessica

Gluhih, Igor

Glukhova, Olga E.

Glynn, Peter

Gmel, Annik Imogen

Gnade, Bruce E.

Gnanasekaran, Karthikeyan

Gnatowski, Andrzej

Gniewosz, Małgorzata

Gobber, Federico Simone

Gobbo, Stefano

Góbi, Sándor

Gockenbach, Ernst

Godfaurd Adjaie, John

Godinez, Fernando

Godino, Leire

Godio, Michele

Godos, Justyna

Godzierz, Marcin

Goehring, Tobias

Goel, Ashish

Goel, Himanshu

Goel, Kanika

Goel, Rahul

Goga, Nicolae

Goh, Guodong

Goh, Shu Ting
Gohari, Soheil

Goins, Justin

Gojani, Ardian B.

Gokaraju, Balakrishna

Goki, Pantea Nadimi

Gola, Arkadiusz

Golabczak, Marcin Andrzej

Golabi, Mohammad H.

Gołaszewska, Małgorzata

Gołaszewski, Jacek

Golcarenarenji, Gelayol

Golchinfar, Behnoush

Goldberg, David

Goldberg, Lynne J.

Goldfarb, Nathaniel

Goldhawk, Donna E.

Golding, Brian

Goldman, Jeremy

Goldmann, Daniel

Goldmann, Kezia

Goldstein, Moshe

Gołębiowski, Piotr

Golec, Piotr

Golewski, Grzegorz Ludwik

Goli, Preetham

Golic-Grdadolnik, Simona

Golijanek-Jędrzejczyk, Anna

Golimowski, Wojciech

Gollie, Jared

Golmohamadi, Hessam

Golob, Aleksandra

Golob, Marjan

Golomazou, Eleni

Golovnin, Oleg Eleni

Golovnin, Oleg K.

Golston, Levi

Goltsov, Alexandr

Golub, Igor

Golub, Mikhail V.

Golubev, Nikolay

Golubev, O. V.

Golubnitschaja, Olga

Golubovic, Nevena

Gomariz-Castillo, Francisco

Gomes, Alberto

Gomes, Ana P.

Gomes, André D.

Gomes, António

Gomes, Catarina

Gomes, Cristina Caramelo

Gomes, Joao 
Gomes, Luciana Calheiros

Gomes, Rahul

Gomez Balderas, Jose Ernesto

Gómez Barrios, Xiomar

Gomez Blas, Nuria

Gómez Bolea, Antonio

Gómez García, Emilio

Gómez Muñoz, Carlos Quiterio

Gomez Rodriguez, Alma

Gómez Suárez, Mónica

Gómez Vela, Francisco A.

Gomez, Antonio

Gómez, José J. Arroyo

Gómez, Marta Matamala

Gomez, Valentín

Gómez, Xabier

Gómez-Abajo, Pablo

Gómez-Alanís, Alejandro

Gómez-Calvet, Roberto

Gómez-Cuba, Felipe

Gómez-de La Cruz, Francisco J.

Gomez-de-Gabriel, Jesus Manuel

Gómez-Déniz, Emilio

Gómez-Galán, Marta

Gomez-Gonzalez, M.

Gómez-Pastora, Jenifer

Gomez-Pau, Alvaro

Gómez-Plaza, Encarna

Gómez-Polo, Miguel A.

Gómez-Ruano, Miguel Angel

Gómez-Salgado, Juan

Gomez-Soriano, Josep

Gómez-Tena, Maria Pilar

Gomez-Tornero, Jose Luis

Gómez-Urquiza, Jose L.

Gömöry, Dušan

Gonalez-Julian, Jesus

Gonçalves, Fernando J.

Gonçalves, João Carlos

Gonçalves, José

Gonçalves, Luís Carlos

Goncalves, Paulo

Gonçalves, Ricardo Rebelo

Gonçalves, Rogério Sales

Gong, Jiawei

Gong, Shaofang

Gong, Zhixiong

Goniewicz, Krzysztof

Gonizzi Barsanti, Sara

Gonnella, Giuseppe

Gonon, Maurice
Gonzales, Ralph Rolly

González Briones, Alfonso

González De La Rosa, Juan-José

Gonzalez De Los Reyes, Rafael Corsino

González Domínguez, Raúl

González Drigo, Ramón

Gonzalez Olivardia, Franchesca G.

González Pino, Iker

González Valero, Gabriel

González, Aurora

González, Begoña

Gonzalez, Camino

González, Carina

González, Elena Labajo

González, Enrique

González, Francisco

González, Hernán

González, Hugo

Gonzalez, Itziar

González, Jose Antonio

González, Luis Ignacio Lopera

Gonzalez, Sandra Castro

González, Sergio

Gonzalez-Ayala, Julian

González-Ayestarán, Rafael

González-Briones, Alfonso

González-Cañete, Francisco Javier

Gonzalez-Casado, Antonio

González-De-Peredo, Ana V.

González-Díaz, Benjamín

González-Díaz, Humbert

González-Domínguez, Pablo Ignacio

González-Doncel, Gaspar

González-Gómez, David

González-Hidalgo, Manuel

Gonzalez-Lezcano, Roberto Alonso

Gonzalez-Longo, Cristina

Gonzalez-Ortega, David

Gonzalez-Parra, Gilberto

González-Prieto, Ángel

González-Rocha, Javier

González-Rovira, Leandro

González-Sálamo, Javier

González-Santamaría, Daniel

González-Suárez, Ana

González-Vila, Álvaro

González-Zamar, Mariana-Daniela

Gonzalo Orden, Hernán

Gonzalo, Jesús

Goo, Byeong-Choon

Goodin, Christopher T. 
Goodrick, Scott L.

Gook, Sergej

Goossen, Keith

Göpfert, Beat

Góra, Jacek

Goraj, Zdobyslaw

Gora-Marek, Kinga

Goranin, Nikolaj

Gorawski, Marcin

Gorbanev, Yury

Gorbaneva, Olga

Gorbatikh, Larissa

Gorbunova, Ella M.

Gordillo, Aldo

Gordon, Wendy R

Gore, Ross

Górecki, Jan

Górecki, Jarosław

Górecki, Krzysztof

Górecki, P.

Górecki, Tadeusz

Górecki, Tomasz

Goreham, Renee

Gori, Antonella

Gorji, Nima E.

Gorkunov, Maxim

Gorlov, Ivan

Gornati, Rosalba

Górniak, Aleksander

Gornicki, Krzysztof

Gorodetsky, Andrei

Gorodetsky, Vladimir

Gorpas, Dimitris

Gorse, Christopher Andrew

Goršek, Andreja

Gorseta, Kristina

Górska, Agata

Górska-Warsewicz, Hanna

Górski, Jarosław

Górski, Marcin

Gorzelanczyk, Piotr

Gorzelańczyk, Tomasz

Gosak, Marko

Gosiewski, Zdzisław

Gospodarek, Janina

Gosselin, Ryan

Goswami, Anwesha

Gosztolya, Gábor

Goto, Kazuya

Goto, Takaharu

Gottwald, Alexander
Götz, Maximilian

Götzendorfer, Babette

Goubej, Martin

Goudail, François

Goudiaby, Venceslas

Gouget, Guillaume

Gouglidis, Antonios

Gouiffès, Michèle

Gouin-Thibault, Isabelle

Goulas, Vlasios

Goursaud, Claire

Goushcha, Oleg

Gouttefarde, Marc

Gouveia, Ricardo M.

Govan, Joseph

Governi, Lapo

Govorunova, Elena G.

Gower, Jim

Gozzi, Caterina

Graba, Marcin

Grabarczyk, Robert

Grabham, Neil

Grabherr, Silke

Grabowska, Sandra

Grabowski, Andrzej

Grabowski, Grzegorz

Grabowski, Marcin

Grabowski, Miroslaw

Grabski, Jakub Krzysztof

Gracin, Davor

Grădinaru, Catalina Mihaela

Gradišek, Anton

Gradziel, Marcin

Grądzki, Rafał

Graef, Frank

Graetz, Jonas

Grafe, Bruno

Gragnani, Gian Luigi

Gragnaniello, Diego

Graham, Katherine

Graham, Matt W.

Graham, Scott

Grajcar, Adam

Grajek, Henryk

Gramlich, Alexander

Gramss, Gerhard

Gramza - Michałowska, Anna

Granado Castro, María Dolores

Granados, Luis Sánchez

Granados-Principal, Sergio

Graña-López, Manuel A. 
Granata, Michele Fabio

Grande, Antonio

Grande, Carlos

Grande, Ernesto

Grande, Francesco

Granero-Gallegos, Antonio

Granitzer, Petra

Grant, David C

Grant, Ryan

Gräser, Axel

Grasley, Zachary C.

Grasso, Alfio Dario

Grasso, Marzio

GRASSO, NIVES

Grattagliano, Ignazio

Grattieri, Matteo

Gratz, Klaus

Grau, Antoni

Grau, Etienne

Gravas, Ioannis P.

Gravenkamp, Hauke

Graziano, Alessandro Di

Graziano, Maria Daniela

Graziano, Sossio Fabio

Gražulis, Saulius

Grazzini, Alessandro

Grbes, Anamarija

Greco, Adriana

Greco, Antonio

Greco, Luca

Grecu, Valentin

Greenhalgh, Stewart A.

Greenman, John

Greer, Andrew I. M.

Greetham, Darren

Grega, Robert

Grego, John

Gregor, Ingo

Gregorcic, Peter

Gregori, Alberto

Gregorová, Magda

Gregov, Goran

Gregurić, Martin

Grekov, Denys

Grella, Marco

Grembecka, Małgorzata

Gremigni, Paola

Greses Huerta, Silvia

Greuling, Andreas

Greviskes, Lindsey

Grewal, Kuljeet Singh
Grgas, Dijana

Gribniak, Viktor

Gric, Tatjana

Grieve, James A.

Grieves, Michael

Grifa, Celestino

Griffin, James

Griffith, Henry

Griffith, Stephen

Griffiths, David

Griffiths, Simon

Grigelionis, Ignas

Grigg, Stephen

Grigolato, Stefano

Grigolini, Paolo

Grigorescu, Elena-Daniela

Grigoropoulos, Gregory

Grigorova-Shtarbeva, Tsvetana

Grigory, Volovik

Grill, Roman

Grilli, Nicolò

Grilo, Carlos

Grimm, Wolf-Dieter

Grimstad, Gustav

Grinbaum, Alexei

Grindeanu, Iulian

Grioli, Giorgio

Grishagin, Vladimir A.

Grishkan, Isabella

Griškevičius, Julius

Grisso, Robert

Gritsenko, Dmitry

Griva, Giovanni Battista

Grivokostopoulou, Foteini

Grm, Aleksander

Grobelna, Iwona

Groch, Paweł

Grochalski, Karol

Grochla, Krzysztof

Grochowicz, Marta

Groendyke, Chris

Grolmusz, Vince

Gromada, Magdalena

Gromisz, Wilhelm

Grondin, Francois

Grondin, Frédéric

Groppi, Flavia

Grose, Christos

Grosenick, Dirk

Grosicki, Greg

Gross, Joshua B. 
Grosse, Mirco

Grossi Bovi Karatay, Graziele

Grossi, Carlota M.

Grossi, Marco

Grossmann, Benjamin

Grossmann, Manfred

Grout, Vic

Groza, Bogdan

Groza, Robert

Grubeša, Ivanka Netinger

Grubeša, Sanja

Grubić Kezele, Tanja

Grubor, Martina

Grudzielanek, Anja Martina

Grueau, Cedric

Gruenwald, Johannes

Gruić, Igor

Grulkowski, Ireneusz

Grum, Janez

Gruna, Robin

Grunwald, Ruediger

Grusch, Michael

Gruss, Łukasz

Gruttner, Kim

Grygierek, Joanna

Gryguć, Andrew

Gryko, Karol

Grym, Jan

Gryning, Mikkel Peter Sidoroff

Grządziel, Jarosław

Grzadziela, Andrzej

Grzechca, Damian

Grzech-Leśniak, Kinga

Grzegorz, Kaczor

Grzegorz, Kokot

Grzegorzek, Martyna

Grzelak, Krzysztof

Grzelak, Małgorzata

Grzelak-Błaszczyk, Katarzyna

Grzesiak, Dariusz

Grześkowiak, Łukasz M.

Grzeszczyk, Stefania

Grznár, Patrik

Grzybek, Dariusz

$\mathrm{Gu}$, Cheng

$\mathrm{Gu}$, Chunju

$\mathrm{Gu}$, Fengshou

Gu, Haichang

$\mathrm{Gu}$, Jianyu

$\mathrm{Gu}$, Jyh-Cherng

$\mathrm{Gu}$, Ning
$\mathrm{Gu}$, Wenting

$\mathrm{Gu}$, Yongjian

Gualdi-Russo, Emanuela

Gualeni, Paola

Gualtieri, Paolo

Guan, Pai-Chen

Guan, Wendy

Guarino, Alfonso

Guarino, Carmine

Guarino, Francesco

Guarino, Stefano

Guastoni, Alessandro

Guatame-Garcia, Adriana

Guazzelli, Elisa

Gubar, Elena

Gubareva, Elena

Guber, Ivo

Gubin, Denis

Gubitosa, Jennifer

Gucciardo, Erika

Gucma, Lucjan

Gucma, Maciej

Gudavalli, Maruti Ram

Gudmundsson, Jon Tomas

Gué, Emilie

Guedda, Mohamed

Guedes, Cristina Miranda

Guédron, Stephane

Gueguen, Philippe

Guentsch, Arndt

Guérard, Guillaume

Guérette, Elise-Andrée

Guernouti, Sihem

Guerra Rosa, Luis

Guerra Yánez, Víctor

Guerra Zubiaga, David

Guerra, Manuela

Guerra, Valentino L. P.

Guerra, Victor

Guerreiro, Joana F

Guerrero, José

Guerrero-Pérez, M. Olga

Guerrero-Rodríguez, José-María

Guerriero, Annie

Guerrini, Lorenzo

Guertler, Anne

Guevara Lopez, Miguel Angel

Guevara, Mario

Guglielmino, Eugenio

Guibal, Eric

Guida, Domenico 
Guidi, Barbara

Guidi, Francesco

Guidotti, Alessandro

Guidotti, Matteo

Guil, Nicolas

Guilherme, Campos

Guillén Gámez, Francisco David

Guillen, Ignacio

Guillén, Maria Dolores

Guillermo Ramón

Guillermo, Palacios Navarro

Guindel, Carlos

Guiné, Raquel

Guiot, Julien

Guisado-Lizar, José-Luis

Guizzi, Guido

Guldiken, Rasim

Gulić, Marko

Guligowska, Agnieszka

Gulin, Oleg E.

Gulkowski, Slawomir

Gullo, Paride

Gulmezoglu, Berk

Gultepe, Ismail

Gumbarević, Sanjin

Gumieniczek, Anna

Guminska, Jolanta

Gumulec, Jaromir

Gumulya, Monica

Gumuscu, Burcu

Gumyusenge, Aristide

Guna, Jože

Gunasegaram, Dayalan R.

Gunasekara, Chamila

Gunawan, Indra

Gundapaneni, Dinesh

Gundić, Ana

Gunia-Krzyżak, Agnieszka

Gunji, Takao

Guńka, Piotr

Gunkel, Matthias

Günther, Matthias

Günther, Tobias

Gunzel, Friederike

Guo, Baoling

Guo, Bin

Guo, Chih-Hung

Guo, Dandan

Guo, Dezhou

Guo, Fenghai

Guo, Hongwei
Guo, Huaqun

Guo, Jianglong

Guo, Jingchuan

Guo, Jiun-in

Guo, Liping

Guo, Na

Guo, Pengfei

Guo, Qiuquan

Guo, Terry Nan

Guo, Xiaolei

Guo, YouGuang

Guo, Yuanhao

Guo, Yue

Guo, Yuntao

Guo, Zhanjun

Gupta, Chetna

Gupta, Chirag

Gupta, Deepa

Gupta, Lalit

Gupta, Pragya Kirti

Gupta, Rishein

Gupta, Shubham

Gupta, Shuchi

Gupta, Siddharth

Gupta, Sneha

Gupta, Sweta

Gupta, Vipul

Gurcan, Önder

Gurka, Martin

Gurupur, Varadraj Prabhu

Gurusinghe, Ranil

Gurwin, Jacek

Gurzhiy, Vladislav

Gusella, Vittorio

Gusev, Michail

Gusev, Nikolai B.

Gusiatin, Zygmunt Mariusz

Guskos, Niko

Guskova, Olga

Gustafsson, Magnus

Gustafsson, Marcus

Gustav, Stalhammar

Gu-Stoppel, Shanshan

Gut, Kazimierz

Gutiérrez Guerrero, Jóse Miguel

Gutiérrez Ramírez, Manuel

Gutiérrez, Enrique

Gutierrez, Eric

Gutierrez, Miguél Ángel

Gutiérrez, Salvador

Gutiérrez, Sarath Mercedes Vega 
Gutiérrez-Martinez, José María

Gutleb, Arno

Guyet, Thomas

Guzanová, Anna

Guze, Sambor

Guzek, Dominika

Guziałowska-Tic, Joanna

Guzik, Agnieszka

Guzik, Maciej

Guzik, Robert

Guzmán, Carlos

Guzmán, Eduardo

Guzmán, Gema

Guzman-Sepulveda, Jose R.

Guzow-Krzemińska, Beata

Guzzini, Alessandro

Gwag, Jin Seog

Gwak, Jeonghwan

Gwamuri, Jephias

Gwiazdowska, Daniela

Gwizdałła, Tomasz M.

Gwozdz, Michal

Gwozdziewicz, Maciej

Gyawali, Buddhi

György, Tóth

$\mathrm{Ha}$, Jand-Hoon

Ha, Jong Moon

Ha, Le Nhu Ngoc Thanh

Ha, William N.

Ha, Yeyoung

Ha, Youn Doh

Haase, Elaine M.

Habel, Michał

Haber, Rodolfo

Haber, Rodolfo E.

Habermehl-Cwirzen, Karin

Habib, Anowarul

Habib, Hany

Habibi, Hamed

Habisreuther, Tobias

Habrat, Witold

Hace, Aleš

Haces Fernandez, Francisco

Hachaj, Tomasz

Hachoł, Justyna

Hackenberger, Davorka K

Hackett, Daniel

Hackl, Christoph M.

Hackney, Philip

Hać-Szymańczuk, Elżbieta

Hadad, Yossi
Hadar, Ofer

Hădărugă, Nicoleta

Hadaś, Tomasz

Haddad, Bouchra

Haddad, Fayrouz

Haddad, Sofiane

Häder, Donat P.

Hadi, Pradita

Hadiwardoyo, Seilendria Ardityarama

Hadjipapas, Avgis

Hadri, Mohammed Salah El

Hadžić, Neven

Hadžić, Vedran

Hadzik, Jakub

Hadzima-Nyarko, Marijana

Haehn, Daniel

Haemmerich, Dieter

Hafezi, Hossein

Haffner, Oto

Hagemann, Thomas

Hagen, Jenny

Hagenfeld, Daniel

Haghighi Osgouei, Reza

Haghshenas, Hamzeh

Hagi, Tatsuro

Hagiwara, Goichi

Hagner-Derengowska, Magdalena

Hahm, Sung Ho

Hahn, Judith

Haidegger, Tamas

Haider, Fasih

Haider, Syed

Haitjema, Han

Hajdu, Miklos

Hajduk, Edmund

Hajej, Zied

Hajek, Manfred

Haj-Hosseini, Neda

Hajilou, Mohammadhadi

Hajiyavand, Amir

Hajj, Ramez

Hajnyš, Jiří

Hájovský, Radovan

Hajtó, Dániel

Hajto, Marek

Hajžman, Michal

Hakak, Saqib

Hakami, Samer

Hakim, Hamed

Hakula, Harri

Hakulinen, Jaakko 
Halada, Gary

Halagarda, Michał

Halbac-Cotoara-Zamfir, Rares

Halder, Amit Kumar

Halder, Sharly Joana

Halicka, Katarzyna

Halilovič, Miroslav

Hall, Duane

Hall, F. Scott

Hall, Joanne V.

Hall, Robyn

Hallberg, Tomas

Hallett, Joseph

Hallil, Hamida

Hallinan, Kevin

Hallmann, Ewelina

Halloran, John

Hally, David

Hałon, Tomasz

Halounová, Lena

Halse, Meghan E.

Halunga, Simona Viorica

Ham, Suyun

Hama, Akira

Hamad, Rebeen Ali

Hamadache, Moussa

Hämäläinen, Joonas

Hamam, Abdelwahab

Hamamoto, Ryuji

Hamann, Kerstin

Hamann, Martine

Hamaza, Salua

Hamdani, Ari

Hamdia, Khader

Hamed, Ali

Hamedi, Hamid Reza

Hameed, Ibrahim A.

Hameed, Khurram

Hameed, Saqib

Hamelin, Benoit

Hamerla, Gordian

Hamida, Mohamed Assaad

Hamidi, Abdallah El

Hamidi, Mohamed

Hamido, Said A.

Hamilton, Andrew R

Hamilton, Chris

Hamlet, Christina

Hammami, Farouk

Hammel, Stephen

Hammer, René
Hammond, Frank L

Hammond, William

Hammoudeh, Mohammad

Hamon, Morgan

Hampel, Daniela

Hampton, Jennifer

Hamza, Ben

Han, Dong

Han, Dongil

Han, Dong-Wook

Han, Hyangsun

Han, Hyoung-Su

Han, Il Song

Han, Jaeyoung

Han, Ji-Hyeong

Han, Jinming

Han, Junghee

Han, Seong-Ik

Han, Won-Sik

Han, Woo-Suck

Han, Xiaohui

Han, Yan (Northwestern University)

Han, Yan (University of Texas)

Han, Yiwei (The University of Mississippi)

Han, Yiwei (University of Mississippi)

Han, Youngsun

Hanafy, Nemany A. N.

Hanak, Tomas

Hanaka, Agnieszka

Hanaor, Dorian

Hanasoge, Srinivas

Hanawa, Hiroki

Hanberry, Brice

Hand, Jon

Handge, Ulrich A.

Handorf, Oliver

Handzlik, Piotr

Haneda, Hajime

Haneklaus, Nils

Hang, Da-Ren

Hangan, Anca

Hanif, Asad

Hankiewicz, Krzysztof

Hannah, Stuart

Hannides, Angelos

Hannouf, Marwa

Hänsel, Andreas

Hansen, Clint

Hansen, Kjetil Falkenberg

Hansen, Uwe

Hanson, Buck 
Hanzelka, Zbigniew

Hanzl, Małgorzata

Hao, Guangbo

Hao, Jiaao

Hao, Jianguo

Hao, Nanjing

Hao, Yong

Haouas, Mohamed

Haq, Anwar Ui

Haque, A B M Tahidul

Haque, Md Emdadul

Haque, Rezwanul

Haque, Sardar Anisul

Haque, Shah Ahsanul

Hara, Hirofumi

Haralick, Robert M.

Harbottle, David

Hardenbicker, Ulrike

Harding, Chris

Harding, Sam

Hardman-Smart, Jonathan

Harih, Gregor

Harik, El Houssein Chouaib

Harirchian, Ehsan

Haririan, Hady

Hariri-Ardebili, Mohammad Amin

Harizanov, Stanislav

Harizi, Walid

Harkat, Houda

Harkness, Patrick

Härmä, Harri

Harmel, Tristan

Harmon, Ian

Haro-Larrode, Marta

Harris, Jason T.

Harris, Merilyn

Harris, Paul

Harrison, Adrian

Harrison, Joshua G.

Harrison, Paul H. M.

Hartmann, Christoph

Hartmann, Michael

Hartmann, Robert

Hartnagel, Hans

Hartwig, Valentina

Hasan, Abdel Fattah R.

Hasan, Abshar

Hasan, Mohammad Mehedi

Hasanzadeh Kafshgari, Morteza

Hascoët, Jean-Yves

Hasegawa, Hideyuki
Hasegawa, Hiromasa

Hasegawa, Miki

Hasegawa, Takaaki

Haselbach, Philipp Ulrich

Hashemi, Shervin

Hashemian, Leila

Hasheminasab, Seyyed Meghdad

Hashimi, Raheel

Hashimoto, Kazuhiko

Hashimoto, Kurumi

Hashimoto, Shigehiro

Hashimoto, Takako

Hashimoto, Takashi

Hashizume, Hiromichi

Hasling, Karen Marie

Haspel, Carynelisa

Hassan Tanveer, Waqas

Hassan, Ahmad

Hassan, Amr Abdulfattah Hausien

Hassan, Emadeldeen

Hassan, Firas

Hassan, Ibrahim

Hassan, Jahan

Hassan, Mahmoud

Hassan, Mohamed

Hassan, Sammer-ul

Hassan, Sherif T. S.

Hassanalian, Mostafa

Hassani, Hossein

Hassanin, Hany

Hassanzadeh, Reza

Hast, Anders

Hata, Hiroo

Hatami, Mohsen

Hatamian, Majid

Hatano, Yuichiro

Hatchett, Andrew

Hatoum, Hoda

Hatt, Mathieu

Hatziadoniu, Constantine

Hatzigeorgiou, George D.

Haugen, Molly

Haupt, Michael

Haurie, Laia

Hausberger, Andreas

Hausman, Sławomir

Haut, Juan Mario

Havaš, Ladislav

Havela, Ladislav

Havenith, Hans-Balder

Havens, Kathryn L 
Havran, Vlastimil

Hawash, Zafer

Hawrot-Paw, Małgorzata

Hawton, Margaret

Hayajneh, Khaled F.

Hayakawa, Takehito

Hayakawa, Yuichi

Hayano, Kimitoshi

Hayashi, Hideki

Hayashi, Hisashi

Hayashi, Jun-ichiro

Hayashi, Kuniyoshi

Hayashi, Tomohiro

Hayashizaki, Kenichi

Hayat, Abdullah Aamir

Hayat, Munawar

Haycock, Bruce

Haylock, Ben

Hazai, László

Hazak, Aaro

Hazari, Md. Rifat

Hazelwood, Richard A.

$\mathrm{He}$, Baojie

$\mathrm{He}$, Cenlin

He, Chengyuan

$\mathrm{He}$, Dongfeng

$\mathrm{He}$, Guanjie

$\mathrm{He}$, Jin

He, Jing Selena

$\mathrm{He}$, Mei

He, Shaoming

$\mathrm{He}$, Siwei

$\mathrm{He}$, Wei

$\mathrm{He}$, Xin

$\mathrm{He}, \mathrm{Yi}$

He, Yinan

$\mathrm{He}$, Yun

He, Zhongqi

Hearn, Gareth James

Hearne, James

Heberger, Karoly

Heberle, Florian

Hecel-Czaplicka, Aleksandra

Hedayati, Reza

Hedjazi, Saman

Hedvičáková, Martina

Hefferan, Kevin Patrick

Hegab, Hussien

Hegedűs, Csaba

Hegmann, Elda

Heiduschka, Peter
Heijungs, Reinout

Heiland, Max

Heilig, Balázs

Heim, Dariusz

Heimgärtner, Rüdiger

Hein, Joachim

Hein, Kyaw

Heinlein, Alexander

Heinrich, Benoît

Heinrich, Philippe

Heinz, Hendrik

Heinze, Christian

Heinzl, Bernhard

Heise, Herbert Michael

Heishman, Aaron

Heisinger, Stephan

Heitkamp, Felix

Heitor, Ana

Heitzler, Magnus

Hejna, Aleksander

Held, Christoph

Helgadóttir, Ásdís

Helle, Mikko

Hellebust, Stig

Hellwig, Michael

Helman, Joanna

Helml, Wolfram

Hely, David

Hemkemeyer, Michael

Hemmatian, Masoud

Hemmersbach, Ruth

Hempel, Martin

Hemprich, Alexander

Hemsel, Tobias

Henderson, Alan D.

Hendriks, Gijs A. G. M.

Hendriks, Max A. N.

Hendrix, Marcel A. M.

Heneberg, Petr

Henesey, Lawrence E.

Hengy, Sebastien

Henke, Markus

Henke, Roberto

Hennebelle, François

Hennebert, Pierre

Hennerici, Michael G.

Henriques, João

Henriques, Rui

Henry, Jérôme

Hensel, Jonas

Henzel, Maciej 
Heo, Jae-Pil

Heo, Jun

Herakovic, Niko

Heras, Jónathan

Heras, Vanessa Las Heras

Herbaut, Nicolas

Herber, Daniel R.

Herbert Acero, José Francisco

Herbst, Andreas

Herceg, Karolina

Herckes, Pierre

Herdzik, Jerzy

Heredia-Juesas, Juan

Herghiligiu, Ionut Viorel

Hering-Bertram, Martin

Herkommer, Alois

Herman, Lukáš

Herman, Przemyslaw

Hermann, Nuno

Hermans, Thomas

Hermerschmidt, Felix

Hermes, Giberti

Hermoso-Orzáez, Manuel Jesús

Hernández Minguillón, Rufino Javier

Hernández Saz, Jesús

Hernandez Sosa, Daniel

Hernández Sosa, José Daniel

Hernández, David Naranjo

Hernandez, Inma

Hernández, José L.

Hernandez, Monica

Hernández, Nacú B.

Hernández, Nicolás

Hernandez-Aldave, Sandra

Hernández-Hernández, César

Hernández-Jiménez, Fernando

Hernandez-Prieto, Miguel A.

Hernando, Antonio

Hernando, David

Herold, Sven

Herraiz, Joaquín López

Herraiz, Miguel

Herrera, Marta

Herrera, Pedro Javier

Herrera-Melián, José

Herrera-Peco, Iván

Herrera-Tapia, Jorge

Herrero, Henar

Herrero, Juan Manuel

Herrero, María Josefa

Herrero, Rolando
Herrero-Durá, Iván

Herreros López, Alberto

Herring, Steven

Herrnsdorf, Johannes

Hersey, Joanna

Herterich, James

Hertweck, Benjamin

Hervada Sala, Carme

Heshmati, Sara

Heshmati-alamdari, Shahab

Heß, Markus

Hess, Paul

Hess, Ronald A.

Hess-Dunning, Allison

Hesselbarth, Anja

Hessler, Steffen

Heunecke, Otto

Heutschi, Kurt

Hevus, Ivan

Hewson, David

Heydari, Azim

Heydari, Rasool

Heydari, Shahram

Heyder, Rodrigo

Hicke, Konstantin

Hidalgo-Herrero, Mercedes

Hideki, Naito

Hidetoshi, Konno

Hieawy, Ahmed

Hiejima, Yusuke

Hiernaux, Stéphane

Hiete, Michael

Higaki, Akinori

Higashi, Tomomi

Higashikawa, Kohei

Hijikata, Yasuto

Hilbrich, Marcus

Hildmann, Hanno

Hilje, Branko

Hill, Daniel

Hiller, Edgar

Hiller, Sven-Jürgen

Hillier, Adrian

Hilloulin, Benoit

Himr, Daniel

Hincapie, Rafael E.

Hinderliter, Brian

Hinds, Gareth

Hingorani, Ramon

Hinkelmann, Knut

Hinman, Samuel 
Hinow, Peter

Hintermann, Beat

Hintermüller, Christoph

Hinz, Hartmut

Hinze, Thomas

Hippler, Rainer

Hippler, Stefan

Hirai, Tomoyasu

Hiraishi, Akira

Hiraishi, Tetsuya

Hirakawa, Yasuyuki

Hirao, Yoshitoshi

Hiraoka, Koichi

Hirata, Kenji

Hirata, Ronaldo

HIRATA, Toshiyuki

Hirayasu, Hirofumi

Hirdaris, Spyros

Hiremath, Nitilaksha

Hirmer, Pascal

Hiroki, Uchiyama

Hirskyj-Douglas, Ilyena

Hita, Idoia

Hitzler, Leonhard

Hiziroglu, Huseyin

Hjelme, Dag Roar

Hjelseth, Eilif

Hladnik, Aleš

Hlaváčová, Irena M.

Hlaváčová, Ivana

Hlavička, Viktor

Hloupis, George

Hnilicka, Frantisek

Ho, Chichun

Ho, Chong Pei

Ho, Danny C. K.

Ho, Eric $S$.

Ho, Hon

Ho, Jörg

Ho, Jun-Won

Ho, Ko-Shan

Ho, Manh-Tung

Ho, Marco

Ho, Thanh Tam

Ho, Yi-Cheng

Hoai Nam, Huynh

Hoang, Khoa Dang

Hoang, Nam

Hoarau, Yannick

Hoath, Stephen

Hobgood, Jay
Hobincu, Radu

Hoblos, Ghaleb

Hochrainer, Thomas

Hochreiner, Armin

Hochstetter, Axel

Hocke, Lia M.

Hodge, Philip

Hodgson, David R. W.

Hodoroaba, Vasile-Dan

Hodulova, Erika

Hoemmen, Mark

Hoenders, Bernhard J.

Hof, Lucas

Hofer, Birgit

Hoff, Inge

Hoffman, Joel

Hoffmann, Andreas

Hoffmann, Klaus H.

Hoffmann, Markus M.

Hoffmann-Eifert, Susanne

Hofman, Tadeusz

Hofman-Caris, Roberta

Hofmann, Ralf

Hogan, Benjamin

Högberg, Lovisa

Hoger, Marek

Höhne, Christian

Höhr, Cornelia

Hoić, Matija

Hojan, Marcin

Hojat Jalali, Himan

Hojjat-Farsangi, Mohammad

Hoła, Bożena

Hoła, Jerzy

Hölbl, Marko

Ho-Le, Thao P.

Holighaus, Nicki

Hollerbach, Rainer

Holliday, Richard

Holló, Gábor

Hollý, Ivan

Holman, Sean

Holmberg, Henrik

Holmes, Christopher

Holonec, Rodica

Holroyd, Steve

Holschemacher, Klaus

Holst, Anders

Holub, Michal

Holubek, Radovan

Holubová, Jana 
Holzinger, Andreas

Homaeigohar, Shahin

Homma, Takujiro

Hommel, Johannes

Homri, Lazhar

Honardoost, Amirmahdi

Honari, Mohammad Mahdi

Honcová, Pavla

Honda, Chikako

Honda, Hiroto

Honda, Katsuhiro

Honda, Masaki

Honda, Yosuke

Hong, Charmgil

Hong, Cheng-Yih

Hong, Chih Chiang

Hong, Guosong

Hong, Ilyoung

Hong, Jang-Eui

Hong, Ji Hyung

Hong, Jin B.

Hong, Joo Young

Hong, Min-Ho

Hong, Seock-Jin

Hong, Seunghwan

Hong, Sungbum

Hong, Tao

Hong, Wei-Chiang

Honório, Túlio

Hontoria, Leocadio

Hooghoudt, Jan-Otto

Hooi Cho, Byoung

Hooke, Christopher J.

Hooshyar, Danial

Hopken, Matthew W.

Horabik, Józef

Horák, Jakub

Horak, Peter

Horbatsch, Marko

Horel, Ágota

Horel, Enguerrand

Horender, Stefan

Hori, Maiya

Horiguchi, Tsuneo

Horla, Dariusz

Horn, Ivo

Hornak, Joseph P.

Horne, Malcolm

Horne, Robert James

Horníček, Leoš

Hornikova, Jana
Hornung, Bastian

Horovistiz, Ana

Horowitz, Robert A.

Horsfall, Alton

Horst, Fabian

Horszczaruk, Elżbieta

Horta Muñoz, Sergio

Hortal, Enrique

Horvat, Barbara

Horvat, Marko

Horváth, Gábor

Horvath, Robert

Horvath, Tomas

Horvatić, Davor

Hosam, El-Ocla

Hošek, Jan

Hoshiba, Takashi

Hoskere, Vedhus

Hosoda, Masahiro

Hosokawa, Atsushi

Hosokawa, Yoshitaka

Hossain, A.K.M. Azad

Hossain, Akter

Hossain, Belayat

Hossain, Eklas

Hossain, Md Alamgir

Hossain, Md Zakir

Hossain, Md. Alamgir

Hossain, Md. Delwar

Hossain, Md. Zakaria

Hossain, Mokarram

Hossan, Mohammad Robiul

Hossein Mardi, Ali

Hosseinali, Farzad

Hosseini Boosari, Seyed Sina

Hosseini Raviz, Seyed Reza

Hosseini, Saeid

Hosseini, Seyed M.H.

Hosseini, Seyed Saeid

Hosseinkhani, Hossein

Hosseinpoor, Masoud

Hosseinzadeh, Mehdi

Hossfeld, Max

Hosur, Vishnu

Hotait, Hassane

Hotos, George N.

Hou, Jia-Woei

Hou, Jie

Hou, Jong-Uk

Hou, Jue

Hou, Lei 
Hou, Shiwang

Hou, Ting-Chao

Hou, Tsung-Chin

Houichi, Kitano

Houska, Jiri

Houstis, Elias

Hovde, Blake T.

Hovem, Jens

Hover, Samantha

Hovorka, Jan

How, Meng-Leong

Howard, Ian

Howell, Bronwyn

Howell, Bryan

Hożyń, Stanisław

Hraska, Jozef

Hrbek, Vojtech

Hrdlicka, Frantisek

Hrechuk, Andrew

Hrenak, Jaroslav

Hribernik, Ales

Hristoforou, Evangelos

Hristov, Ilian

Hromada, Martin

Hromadka, Vit

Hron, Karel

Hrzina, Pavel

Hsia, Shao-Yi

Hsia, Shih-Min

Hsiang, Hsing-I

Hsiao, George

Hsiao, Hui-Hsin

Hsiao, Pai-Yi

Hsiao, Shen-Fu

Hsiao, Shih-Wen

Hsiao, Wen-Tse

Hsieh, Chang-Wei

Hsieh, Cheng-Hsiung

Hsieh, Chiu-Fan

Hsieh, Lu-Sheng

Hsieh, Ping-Cheng

Hsieh, Raymond J.

Hsieh, Tung-Hsun

Hsieh, Yao-Ching

Hsieh, Yi-Chen

Hsi-Jen James, Yeh

Hsu, Chia-Jui

Hsu, Ching-Chi

Hsu, Fu-Chiun

Hsu, Heng-Tung

Hsu, Hsien-Pin
Hsu, Jin-Chen

Hsu, Jui-Ting

Hsu, Kuan-Lun

Hsu, Ming-Hua

$\mathrm{Hsu}$, Ming-Ying

Hsu, Po-Chun

Hsu, Shan-hui

Hsu, Timothy

Hsu, Wei-feng

$\mathrm{Hsu}$, Wei-Hsuan

Hsueh, Ming-Chun

Hsueh, Ya-Hsin

$\mathrm{Hu}$, Guoping

$\mathrm{Hu}$, Hui

$\mathrm{Hu}$, Jianjun

$\mathrm{Hu}$, Jianli

$\mathrm{Hu}$, Jong Wan

$\mathrm{Hu}$, Junling

$\mathrm{Hu}, \mathrm{NianZe}$

$\mathrm{Hu}$, Peng

$\mathrm{Hu}$, Sijung

$\mathrm{Hu}$, Tangao

$\mathrm{Hu}$, Tao

$\mathrm{Hu}$, Taobo

$\mathrm{Hu}$, Wei

$\mathrm{Hu}, \mathrm{Xi}_{\mathrm{i}}$

$\mathrm{Hu}$, Xianfeng

$\mathrm{Hu}$, Xiaosu

$\mathrm{Hu}$, Xiaoyu

$\mathrm{Hu}$, Yang

$\mathrm{Hu}$, Yaxi

$\mathrm{Hu}$, Yinlin

$\mathrm{Hu}$, Yu Hen

Hua, Gang

Hua, Jie

Huang, Anthony

Huang, Boyang

Huang, Chaoran

Huang, Chih-Ling

Huang, Ching-Hsu

Huang, Chung-Chi

Huang, Chun-Ying

Huang, Guangyan

Huang, Hailong

Huang, Haiyan

Huang, Hantao

Huang, Heng-Li

Huang, Henry D.

Huang, Hongxin

Huang, Hsin-Haou

Huang, Hung-Chung 
Huang, Hung-Pin

Huang, Jen-Wei

Huang, Jih-Jeng

Huang, Jingfeng

Huang, Jong-Chin

Huang, Kaiwu

Huang, Keng-Shiang

Huang, Kuo-Cheng

Huang, Long-Sheng

Huang, Ming

Huang, Ming-Shyan

Huang, Pei-Chi

Huang, Po-Hsun

Huang, Qijin

Huang, Shaoguang

Huang, Sheng

Huang, Shian-Chang

Huang, Shih-Chang

Huang, Shuan-Yu

Huang, Shyh-Chour

Huang, Sunan

Huang, Tse-Hung

Huang, Tzu-Hao

Huang, Weichuan

Huang, Weimin

Huang, Weixin

Huang, Wen-Chin

Huang, Wu-Jang

Huang, Xiao

Huang, Xiaodi

Huang, Xiaoxue

Huang, Yanbo

Huang, Yifeng

Huang, Yishuo

Huang, Yi-Ting

Huang, Yuhan

Huang, Yu-Hsi

Huang, Zhaorong

Huang, Zhirong

Huba, Mikuláš

Huber, Milosh

Huber, Miłosz

Hubicki, Christian M.

Hübl, Johannes

Hübler, Clemens

Hudişteanu, Valeriu Sebastian

Huelva, Marta Molina

Huerta-Angeles, Gloria

Huertas Abril, Cristina A.

Huertas-Leyva, Pedro

Huesing, Mathias
Hughes, Michael

Hugo, Sol

Hugtenburg, Richard

Huh, Jungwon

Huh, Jun-Ho

Hui, Jie

Hui, Teo Tee

Hui, Xuan

Huignard, Jean-Pierre

Huilier, Daniel

Hulea, Mircea

Huljenić, Darko

Hulka, Karel

Hulsey, Leroy

Hülsmann, Michael

Humbert, Maria V.

Humelnicu, Doina

Humphries, Brock

Hunek, Wojciech

Hung, Ching

Hung, Ho-Lung

Hung, Jui-chung

Hung, Ling-Hong

Hung, Pui-yan

Hung, Shao-Wen

Hung, Yin-Chun

Hung, Yu-Han

Hung, Yung-Tse

Hunicz, Jacek

Hunt, Hayley

Hunter, Charles T.

Huo, Yiming

Huot, Jacques

Huptych, Michal

Hur, Kyeong

Hur, Sun

Hurst, William

Hurtík, Petr

Hurwitz, Shepard

Husár, Jozef

Huscroft, Joseph

Husev, Oleksandr

Husmann, Anke

Hussain, Fatima

Hussain, Ghulam

Hussain, Jamil

Hussain, Muhammad Imtiaz

Hussain, Muhammad Majid

Hussain, Niamat

Hussain, Shahid

Hussain, Zahir M. 
Hussaini, Haizal

Hussanan, Abid

Hussein, Husam

Hussein, Moustafa

Hussein, Ramy

Hussein, Sarfaraz

Hutchinson, John W.

Hutt, Axel

Huttunen-Lenz, Maija

Huyen, Tran Thi Thuong

Huyer, Waltraud

Huynh, Hoai Nam

Huynh, Man

Huynh, Thanh-Canh

Huynh-The, Thien

Huzar, Zbigniew

Hwang, Cheol-Hong

Hwang, Chi-Hung

Hwang, Dong-Hwan

Hwang, Gi-Byoung

Hwang, Hsiu-Ying

Hwang, Jae Youn

Hwang, Jun-Dar

Hwang, Keum-Cheol

Hwang, Myun Joong

Hwang, Shyh-Shin

Hwang, Soon Jung

Hwang, Taek Yong

Hwang, Woonbong

Hwang, Yin-Tsung

Hwang, Youngbae

Hwang, Yuhoon

Hwangbo, Hyunwoo

Hwu, Jenn-Gwo

Hybská, Helena

Hyde, Peter

Hyk, Wojciech

Hyla, Tomasz

Hyrynsalmi, Sami

Hysek, Stepan

Hýsek, Štěpán

Hyun, Eugin

Hyun, Sunghyup Sean

Iacone, Roberto

Iacono, Mauro

Iaculli, Flavia

Iametti, Stefania

Iammarino, Marco

Ianchis, Raluca

Iancovici, Mihail

Iannace, Gino
Iannazzo, Daniela

Ianniello, Roberto

Iannuzzi, Clara

Ianora, Adrianna

Ianos, Ioan

Iasur-Kruh, Lilach

Iatsunskyi, Igor

Iban, Antolín Lorenzana

Ibáñez-González, María J.

Ibáñez-Vera, Alfonso Javier

Ibarlucea, Bergoi

Ibl, Martin

Ibrahim, Ahmed

Ibrahim, Ali

Ibrahim, Fandi

Ibrahim, Hamdy

Ibrahim, Mohamed Ali

Ichchou, Mohamed Najib

Iclodean, Calin

Idehen, Ikponmwosa

Idir, Rachida

Iebba, Valerio

Ientile, Riccardo

Ieracitano, Cosimo

Ierapetritou, Marianthi

Iero, Demetrio

Iervolino, Giuseppina

Ietto, Fabio

Ifelebuegu, Augustine

Iftikhar, Nadeem

Igarashi, Go

Igawa, Manabu

Igawa, Takeshi

Igaz, Rastislav

Iglesias, Carlos Angel

Iglesias-Juez, Ana

Ignacio Arribas, Juan

Ignaciuk, PrzemysŁaw

Ignat, Anca

Ignat, Maria

Ignatova, Tetyana

Igor, Gornushkin

Igor, Zagorodnev

Igual, Jorge

Igual, Marta

Iguchi, Akira

Igwemezie, Victor

Ihantola, Sakari

Iijima, Kazutoshi

Iio, Jun

Iitani, Kenta 
Ijaz, Muhammad Fazal

IJzerman, Wilbert L.

Ikäheimo, Jussi

Ikarashi, Kikuo

Ikeda, Brian

Ikehashi, Tamio

Ikerionwu, Charles

Ikram, Awais

Il'ina, Marina V.

Ilaš, Janez

Ilba, Mateusz

Ileana Corcau, Jenica

Ileš, Šandor

Ilewicz, Grzegorz

Iliadis, Lazaros

Ilias, Sakellariou

Ilies, Nicoleta

Iliescu, Mihaiela

Ilieva, Galina

Ilinčić, Petar

Ilinova, Alina

Illia, Dobryden

Ilnicka, Anna

Iluk, Artur

Ilyakov, Evgeny V.

Im, Hyeonseung

Im, Tae Ho

Imai, Jun

Imai, Tomoaki

Imam, Tasadduq

Imbery, Terence A.

Imlau, Mirco

Imperatore, Roberta

Imperatore, Stefania

Imran, Ali Shariq

Imtiaz, Masudul Haider

Imtiaz, Mohammad

Imtiaz, Syed Anas

In, Jung Bin

Inaba, Atsushi

Inaba, Masafumi

Inalpolat, Murat

Inard, Christian

Inayat, Amer

Inazumi, Shinya

Inchingolo, Francesco

Infantes, Lourdes

Ingaldi, Manuela

Ingalls, Brian

Ingle, Atul

Inglese, Gabriele
Ingold, Gert-Ludwig

Ingrassia, Michela

Ingrassia, Tommaso

Inoue, Go

Inoue, Kazuya

Inoue, Kyo

Inoue, Ryo

Inoue, Shuhei

Insua-Arévalo, Juan Miguel

Intaratep, Nanyaporn

Intini, Paolo

Intzes, Ioannis

Inuso, Giuseppina

Invernizzi, Stefano

Ioana, Cornel

Ioan-Lucian, Popa

Ioannidis, Georgios

Ioannidou, Alexandra

Ioannidou, Melina P.

Ioannis Manousakas, Manousos

Ioannou, Stelios

Iocca, Oreste

Iodice, Paolo

Ion, Ion V.

Ion, Rodica-Mariana

Ionel, Chirica

Ionel, Ioana

Ionescu, Elena Rodica

Ionescu, Laurentiu Mihai

Ionescu, Valeriu Manuel

Ionete, Eusebiu Ilarian

Ionita, Anca Daniela

Ionita, Petre

Ionita, Silviu

Ioniţă, Valentin

Ionov, Leonid

Iordanova, Ivanka

Iorizzo, Massimo

Iosif, Andras

Iosifidis, Michael

Iotti, Eleonora

Iovieno, Michele

Ipšić, Ivo

Iqbal, Asif

Iqbal, Hafiz M. N.

Iqbal, Mudassir

iqbal, nasir

Iqdiam, Basheer

Irie, Mitsuteru

Irimescu, Adrian

Iris, Cagatay 
Irshad, Ahamed

Irulegi, Olatz

Irusta, Unai

Isac, Luminita

Isalgue, Antonio

Isanaka, Sriram Praneeth

Isasi, José Ramón

Isbrandt, Marek

Isern, David

Isezaki, Takashi

Isgrò, Francesco

Ishak, Shafiq

Ishaq, Isam

Ishihara, Keiichi N.

Ishihara, Kengo

Ishii, Isao

Ishikawa, Jun

Ishikawa, Satoshi

Ishikawa, Tadahiko

Ishitsuka, Yosuke

Ishiwatari, Mikio

Ishizuka, Hiroki

Isigonis, Panagiotis

Isik, Sabahattin

Islam, Ehsan Sabri

Islam, Mazharul

Islam, Md Rabiul

Islam, Md Rashadul

Islam, Md Saiful

Islam, Md. Zohurul

Islam, Mohammad Nurul

Islam, Mohammad S.

Islam, Mohammad Zahirul

Islam, Muhammad Aminul

Islam, Raihan Ul

Islam, S. M. Rakiul

Islam, S. M. Riazul

Islam, Saiful

Island, Joshua

Ismail, Umair

Isoaho, Noora

Isola, Daniela

Isola, Gaetano

Iso-Touru, Terhi

Istrati, Denis

Istvanek, Matej

Isufi, Brisid

Isvoranu, Dragos

Itani, Sarah

Itaya, Yoshinori

Itina, Tatiana E.
Ito, Akinori

Ito, Kiyohiro

Ito, Naoki

Ito, Shosuke

Ito, So

Ito, Tadashi

Ito, Yoshihiro

Itoh, Tamitake

Itoyama, Katsutoshi

Itrić, Katarina

Ityokumbul, Mku Thaddeus

Iudice, Ivan

Iungo, Giacomo Valerio

Ivan, Pires

Ivan, Vladimir

Ivančević, Darko

Ivanescu, Mircea

Ivañez, Inés

Ivanišević, Ana

Ivanishchev, Aleksandr V.

Ivannikov, Alexander

Ivanov Cholakov, Slav

Ivanov, Hristo

Ivanov, Ilia N.

Ivanov, Ovidiu

Ivanov, Valentin

Ivanov, Vladimir K.

Ivanova, Alla V.

Ivanova, Dimka I.

Ivanova, Krasimira Borislavova

Ivanova, Svetlana

Ivanovska, Tatyana

Ivashchenko, Olena

Ivić, Stefan

Ivica, Anja

Ivorra, Eugenio

Ivorra, Salvador

Ivshina, Irena

Ivsic, Branimir

Ivšinović, Josip

Iwakoshi, Takehisa

Iwamoto, Takeshi

Iwan, Agnieszka

Iwan, Stanislaw

Iwanowski, Marcin

Iwański, Marek

Iwański, Mateusz

Iwata, Shinya

Iyer, Janaki

Iza, Felipe

Izadgoshasb, Iman 
Izadi Moud, Hashem

Izadifar, Zohreh

Izaguirre, Alberto

Izaguirre, Alberto Cordoba

Izawa, Kazuhiro P.

Izawa, Tetsuya

Izonin, Ivan

Izquierdo, María Concepción Pilar

Izquierdo-Fuente, Alberto

Izydorczyk, Jacek

Izzetti, Rossana

Izzo, Luana

Izzo, Riccardo

Jaba, Elisabeta

Jabarulla, Mohamed Yaseen

Jabba, Daladier

Jaber, Ali Malik

Jaber, Mona

Jablan, Jasna

Jabłońska, Joanna

Jabłoński, Paweł

Jabraoui, Hicham

Jacak, Janusz

Jacak, Lucjan

Jacak, Witold Aleksander

Jack, Rachael E.

Jackowska-Strumiłło, Lidia

Jackson, David R.

Jackson, Mark

Jacobsen, Elisabeth

Jacoby, Margarite

Jacques, Angela

Jaczewski, Mariusz

Jadidi, Kazem

Jadidi, Mehdi

Jafari, Azadeh

Jafari, Mohammad

Jafari, Raheleh

Jafari, Soheil

Jafarov, Elchin E

Jafferis, Noah T.

Jaganathan, Arun

Jagannath, D.J.

Jäger, Georg

Jägerbrand, Annika K.

Jagiela, Mariusz

Jagoda, Marcin

Jagodnik, Vedran

Jagodzinski, Filip

Jahan, Suchana Akter
Jahnke, Gizella

Jahromi, Hossein

Jaime, Marcelo

Jain, Akhilesh

Jain, Nitin

Jaiswal, Amit

Jajac, Nikša

Jakab, Endre

Jakica, Nebojsa

Jakieła, Sławomir

Jakimowicz, Aleksander

Jakóbczak, Dariusz Jacek

Jakóbczyk, Lech

Jakóbik - Kolon, Agata

Jakobsen, Ove

Jakobson, Claudio

Jakobsson, Jonas

Jakovljevic, Gordana

Jakowluk, Wiktor

Jakubczyk-Gałczyńska, Anna

Jakubik, Wiesław P.

Jakubowski, Jacek

Jakubowski, Marek

Jakubowski, Robert

Jakus, Damir

Jalal, Ahmed

Jalali, Mandana

Jalan-Sakrikar, Nidhi

Jalava, Katri

Jalil, Bushra

Jaliu, Codruta

Jaliu, Codruta Ileana

Jamalabadi, Mohammad Yaghoub

Abdollahzadeh

Jamaludin, Mohd Syahmi

Jambrosic, Kristian

Jameel, Shoaib

James, Corey M.

James, Lois

James, Rob S.

James, Robin

Jamil, Faisal

Jamoos, Ali

Jamróz, Piotr

Jamroziak, Krzysztof

Jamshidi, Ali

Jamshidpour, Ehsan

Jan Laskowski, Łukasz

Jan, Chyan-Long

Ján, Vozár

Jana, Atanu 


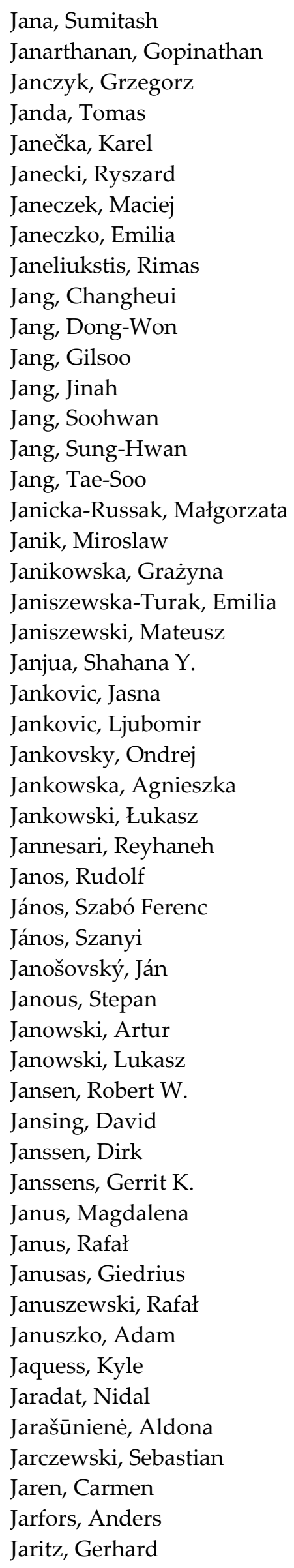

Jana, Sumitash
Janarthanan, Gopinathan

Jana, Sumitash
Janarthanan, Gopinathan

Jaromin, Anna

Jaroslav, Vrchota

Jaroszewicz, Zbigniew

Jaroszewski, Maciej

Jarvis, Daniel

Jarzebski, Maciej

Jarzyna, Jadwiga A.

Jasenek, Jozef

Jasiewicz, Marcin

Jasińska, Elżbieta

Jasińska, Izabela

Jasinskas, Algirdas

JASINSKI, Jaroslaw Jan

Jasiński, Marek

Jasiński, Mariusz

Jasinski, Michal

Jasiński, Tomasz

Jasionowski, Robert

Jasiul, Bartosz

Jasiulewicz, Malgorzata

Jasiulewicz-Kaczmarek, Malgorzata

Jaskiewicz, Marek

Jaskulski, Roman

Jaśniok, Mariusz

Jastrzab, Renata

Jastrzebska, Agnieszka

Jastrzębska, Magdalena

Jastrzębska, Małgorzata

Jasutiene, Ina

Jaszczur-Nowicki, Jarosław

Jauregui, Catherine

Javadian, Alireza

Javaherchi, Teymour

Javaid, Saher

Javed, Hassan

Javed, Muhammad

Jawecki, Bartosz

Jaworek- Korjakowska, Joanna

Jaworska, Beata

Jaworska, Tatiana

Jaworski, Piotr

Jayamohan, Harikrishnan

Jayaraman, Sundararajan

Jayasinghe, Laddu Bhagya

Jayathunga, Sadeepa

Jazaei, Robabeh

Jean-Fulcrand, Annelise

Jebelli, Ali

Jedrzejczak, Wiktor

Jędrzejewska, Agnieszka

Jedrzejewska-Szczerska, Małgorzata 
Jedrzejowicz, Joanna

Jee, Yong-Seok

Jeen, Sung-Wook

Jeerapan, Itthipon

Jeevanandam, Jaison

Jegede, Abiodun

Jegelevičius, Darius

Jehel, Pierre

Jeleński, Tomasz

Jelušič, Primož

Jen, Chun-Ping

Jen, Yi-Jun

Jena, Himanshu Sekhar

Jencova, Vera

Jendželovský, Norbert

Jeng, Dong-Sheng

Jeng, Jeng-Ywan

Jensen, Andrew

Jensen, Mikael

Jenuš, Petra

Jeon, Heung Seok

Jeon, Jun-Cheol

Jeon, Junpyo

Jeon, Ju-Won

Jeon, Kwang Myung

Jeon, Mansik

Jeon, Minhyon

Jeong, Byung Yong

Jeong, Chang Kyu

Jeong, Dong Hyun

Jeong, Eui Seob

Jeong, Gi-young

Jeong, Hae-Duck Joshua

Jeong, Himchan

Jeong, Injae

Jeong, Jaewook

Jeong, Jin Su

Jeong, Jin-Woo

Jeong, Keun-Yeong

Jeong, Myeong-Hun

Jeong, Okran

Jeong, Se Kyoo

Jeong, Seon-Yong

Jeong, Yeonung

Jeong, Yong-jin

Jeong, Young-Seob

Jeremias, Filipe Telmo

Jerez-Mesa, Ramon

Jerkovic Stil, Vedrana

Jerkowić, Igor

Jermakowicz-Bartkowiak, Dorota
Jerman, Igor

Jerzak, Malgorzata Maria

Jerzak, Wojciech

Jerzykiewicz, Maria

Jesenik, Marko

Jeske, Tim

Jessel, Jean-Pierre

Jesus, Goncalo

Jewiarz, Marcin J.

Jezowski, Pawel

Jha, Ajit

Jhan, Jyun-Ping

Jhatial, Ashfaque Ahmed

Jheng, Yu-Ming

Jhon, Young Min

Ji, Aimin

Ji, Dongxu

Ji, Guomin

Ji, Jian

Ji, Kyunghee

Ji, Siyuan

Ji, Wooseok

Ji, Xiaoxu

Ji, Yisi D.

Jia, Chen

Jia, Chunrong

Jia, Guanbo

Jia, Mengda

Jia, Mingtu

Jia, Tony

Jia, Xiaolu

Jia, Yaoyao

Jia, Zixian

Jian, Wurong

Jiang, Cheng

Jiang, Dongyun

Jiang, Fan

Jiang, Liben

Jiang, Long

Jiang, Richard

Jiang, Ruinian

Jiang, Saihua

Jiang, Shan

Jiang, Shaofeng

Jiang, Xianta

Jiang, Xiaoli

Jiang, Zhengyi

Jílková, Veronika

Jillella, Dinesh

Jimenez Alonso, Javier Fernando

Jiménez Ballesta, Raimundo 
Jiménez Cantizano, Ana

Jiménez López, Jesús

Jiménez Millán, Juan

Jimenez, Emilio

Jiménez, Francisco Marco

Jiménez, José Ramón

Jimenez, Ricardo

Jiménez-Alonso, Javier Fernando

Jimenez-Bescos, Carlos

Jiménez-Fernández, Eduardo

Jiménez-Herranz, José Miguel

Jimenez-Hornero, Jorge E.

Jimenez-Olmedo, Jose M.

Jin, Chenglu

Jin, Congrui

Jin, Hye Jin

Jin, Hyo-Eon

Jin, Jiangliang

Jin, Jing

Jin, Miaomiao

Jin, Wencheng

Jin, Yifei

Jin, Zhanpeng

Jindo, Keiji

Jing, Lei

Jing, Ran

Jinno, Yohei

Jirásko, Daniel

Jirau-Colón, Héctor

Jiu, Jinting

Jo, Hwanju

Jo, Sae Byeok

Jo, Young Chang

Jo, Young Hoon

Jo, Young Min

João Ramalhosa, Maria

João, Silva

Jobst, Markus

Jochymczyk-Woźniak, Katarzyna

Joda, Tim

Jødal, Lars

Joeckel, R.M.

Johannes, Kiefer

Johannssen, Arne

Johansen, Kerstin

Johansson, Christer

Johansson, Örjan

Johanyák, Zs. Csaba

Johnsen, Joakim

Johnson Shepherd, Jami

Johnson, Eric
Johnson, Eric N.

Johnson, Jeremy

Johnson, Xenie

Johnston, Benjamin

Jollans, Lee

Jolly, Pascal

Jolly, Pawan

Jombo, Gbanaibolou

Jona, Gyorgy

Jonak, Józef

Jonda, Ewa

Jonek-Kowalska, Izabela

Jones, Albert T.

Jones, Elena

Jones, Eriita

Jones, Jeanne

Jones, Karl O.

Jones, Mark W.

Jones, Philip

Jones, Thomas

Jonmohamadi, Yaqub

Jonuscheit, Joachim

Joo, Ki-Nam

Jopek, Hubert

Jordán, Manuel

Jordana, Rafael

Jordao, Luisa

Jörg, Beckmann

Jorge Evangelista, Ana Catarina

Jornaz, Abdelmonaem

Jorquera-Chavez, Maria

Jorquera-Lucerga, Juan José

José Ortiz, María

Jose, Sanchez Ramos

Josep, Arnal

Joseph, Wout

Joshi, Gyanendra Prasad

Joshi, Ravindra

Joshi, Sameer

Joshi, Suraj

Jošić, Hrvoje

Jot, Jean-Marc

Jotautiene, Egle

Jothimani, Dhanya

Joubaneh, Eshagh Farzaneh

Jouko, Tuomisto

Joukov, Vladimir

Jourdan, Franck

Jović, Jurica

Jovicic, Vojislav

Jovicic, Vojkan 
Jovid, Rakhmonov

Jozaghi, Ali

Jozef, Stoklosa

Józefczak, Arkadiusz

Józsa, Viktor

Jóźwiak, Arkadiusz

Jozwiak-Niedzwiedzka, Daria

Jóźwiak-Niedźwiedzka, Daria

Jóźwiakowski, Krzysztof

Jóźwik, Paweł

$\mathrm{Ju}, \mathrm{Bu}-\mathrm{Seog}$

Ju, Shen-haw

Juan, Andrew

Juan, Carlos G.

Juang, Jia-Yang

Juan-Valdes, Andres

Jubeau, Sébastien

Jucius, Dalius

Judek, Slawomir

Judge, Mark

Juez, Carmelo

Jug, Jasmin

Juhasz, Janos

Juidías, Emilio Ramírez

Jukic Spika, Maja

Jukić, Željko

Juknelevičius, Romualdas

Julian, Fernando

Julian, Ignacio

Julio, Bastos Arrieta

Jun, Han Jong

Jun, Sang Beom

Jun, Se-Ran

Jun, Yongseok

Junakova, Natalia

Jung, Chanho

Jung, Christiane

Jung, Christopher

Jung, Dae-Yi

Jung, Dongwhi

Jung, Eun Mi

Jung, Gwang-Pil

Jung, Haejoon

Jung, Haesung

Jung, Hosang

Jung, Ho-Sup

Jung, Jaesoon

Jung, Jae-Yoon

Jung, Jaeyoung

Jung, Jangwook

Jung, Jason J.
Jung, Jinmu

Jung, Jinwoo

Jung, Kyung Hwan

Jung, Kyung-Hye

Jung, Seok-Ki

Jung, Sunghun

Jung, Sunghwan (Sunny)

Jung, Yoonsung

Jung, Young-Dae

Junghans, Lars

Junker, Rune Grønborg

Junne, Stefan

Juntune, Joyce

Juodkazis, Saulius

Juráček, Miroslav

Jurado, Juan M.

Juráš, Peter

Jurcek, Ondrej

Jurcevic, Marko

Jurczak, Robert

Jureczko, Mariola

Jurek, Michał

Jürgen, Bär

Jurić, Slaven

Jurkowska, Nadzieja

Jurman, Giuseppe

Jusas, Vacius

Juśkiewicz, Jerzy

Juszczyk, Michał

Juszczyk, Piotr

Jůzl, Miroslav

Kabala, Zbigniew

Kabalyk, Kirill

KABASHI, Naser

Kabiersch, Grit

Kabir, Golam

Kabiri, Shervin

Kabošová, Lenka

Kaburlasos, Vassilis G.

Kacetl, Jaroslav

Kachanov, Vladimir Klimentievich

Kacianauskas, Rimantas

Kačík, František

Kacimi, Mouna

Kącka-Zych, Agnieszka

Kačur, Ján

Kaczmarczyk, Jarosław

Kaczmarczyk, Michał

Kaczmarek, Adam L.

Kaczmarek, Katarzyna

Kaczmarek, Małgorzata T. 
Kaczmarek, Michal

Kaczmarek, Sławomir

Kaczmarek, Wojciech

Kaczmarek-Kędziera, Anna

Kaczorek, Dobrosława

Kaczorek, Ewa

Kaczor-Urnanowicz, Karolina Elzbieta

Kaczyński, Paweł

Kadam, Avinash

Kádár, Csilla

Kadar, Manuella

Kaddoum, Georges

Kadel, Rajan

Kadela, Marta

Kadela-Tomanek, Monika

Kadem, Lyes

Kadi, Nawar

Kadife, Elif

Kadir, Tariq

Kadirova, Seher

Kadlimatti, Ravi

Kadokawa, Jun-ichi

Kaese, Sven

Kaewunruen, Sakdirat

Kafarski, Pawel

Kafexhiu, Fevzi

Kagawa, Masaharu

Kagemoto, Hiroshi

Kageshima, Yosuke

Kagiyama, Nobuyuki

Kagramanov, Georgiy

Kahaki, Seyed M. M.

Kahl, Stefan

Kahle, Eckhard

Kahng, Sungtek

Kaimakamis, Evangelos K.

Kairytė, Agnè

Kaisarly, Dalia

Kajánek, Pavol

Kajanova, Martina

Kajimoto, Hiroyuki

Kajiwara, Yusuke

Kakanakov, Nikolay

Kakkassery, Vinodh

Kaklauskas, Arturas

Kaklauskas, Gintaris

Kaklis, Kostas

Kalambura, Sanja

Kalantari, Mohsen

Kalatehjari, Roohollah

Kalayci, Tuna
Kalbarczyk, Robert

Kalenik, Marek

Kales, Stefanos

Kaletova, Tatiana

Kaliakatsos-Papakostas, Maximos

Kalibatiene, Diana

Kalinchuk, Valery V.

Kalinichenko, Antonina

Kalinin, Alexandr

Kalinitchenko, Valery

Kalinowska-Wichrowska, Katarzyna

Kalinowski, Krzysztof

Kalinowski, Maciej

Kalisch, Holger

Kalisz, Dorota

Kalisz, Piotr

Kaliva, Maria

Kalivoda, Jan

Kalkan, Ali Kaan

Kalliokoski, Matti

Kallioniemi, Elisa

Kallweit, Stephan

Kalogeropoulos, Kleomenis

Kalogiannakis, Michail

Kalogirou, Andreas S.

Kalous, Lukáš

Kalua, Amos

Kałuża, Marta

Kałużny, Jarosław

Kalvet, Tarmo

Kalyana Sundaram, Ramalingam Venkat

Kalyandurg, Pruthvi Balachandra

Kalyvas, Nektarios

Kam, Moshe

Kam, Rothman

Kamalanathan, Manoj

Kamali, Negar

Kamamichi, Norihiro

Kamanina, Natalia

Kamat, Vineet R.

Kamath, Divya

Kambezidis, Harry

Kambhampati, Chandra

Kambiz, Tehrani

Kamel, Mohamed A.

Kamel, Mohammed Saad

Kamenar, Ervin

Kamer, Angela

Kameyama, Atsushi

Kaminaris, Stavros D.

Kamińska, Joanna A. 
Kaminska-Chuchmala, Anna

Kaminski, Daniel M.

Kamiński, Marcin

Kaminski, Piotr

Kamisalic, Aida

Kammer, Julien

Kamola, Mariusz

Kamoutsis, Athanasios

Kampa, Adrian

Kampczyk, Arkadiusz

Kampelis, Nikos

Kamperidou, Vasiliki

Kämpfer-Homsy, Alexandra

Kamphuis, Nathan

KAMPITSIS, Georgios

Kampouris, Thodoros E.

Kamps, Jan Henk

Kamshilin, Alexei

Kanagawa, Tetsuya

Kanakaris, Venetis

Kanakubo, Toshiyuki

Kanamoto, Toshiki

Kanarachos, Stratis

Kanaujiya, Jitendra

Kanavos, Andreas

Kanazaki, Masahiro

Kanbayashi, Toru

Kanbur, Barış Burak

Kancharla, Abhilash

Kanczler, Janos

KANDA, Akinobu

Kandadai, Nirmala

Kandelbauer, Andreas

Kander, Thomas

Kandukuri, Surya Teja

Kandylis, Panagiotis

Kaneko, Toshiro

Kanel, Gennady

Kanellopoulos, Christos

Kanemura, Naohiko

Kanerva, Mikko

Kang, Bongsoon

Kang, Byeongkeun

Kang, Dae-Ki

Kang, Dong Jin

Kang, Dong-Won

Kang, Gu

Kang, Gu Eon

Kang, Hee-Jun

Kang, Hongki

Kang, Hyunchul
Kang, Hyun-Kyung

Kang, Hyunwoo

Kang, Jaeyoung

Kang, James Jin

Kang, Jeeun

Kang, Jeon Woong

Kang, Jeon-Young

Kang, Jianming

Kang, Jidong

Kang, Joo H.

Kang, Jun

Kang, Junsu

Kang, Kai

Kang, Kyungtae

Kang, Li-Wei

Kang, Myungkoo

Kang, Shinhyu

Kang, Song-Lak

Kang, Sung-Hoon

Kang, Taeghyun

Kang, Yang Jun

Kang, Youngsoo

Kang, Yunqing (Kevin)

Kangas, Teija

Kania, Henryk

Kania, Krzysztof

Kania, Tomasz

Kanianska, Radoslava

Kannan, Rangasayee

Kanngießer, Birgit

Kanno, Takahiro

Kano, Naoki

Kansake, Bruno

Kansal, Ankush

Kantake, Masato

Kantar, Emre

Kantarci, Burak

Kantartzis, Nikolaos V.

Kaňuch, Ján

Kanwal, Nadia

Kao, Da-Yu

Kao, Hui-Ling

Kao, Yung-Chou

Kapcia, Konrad Jerzy

Kapczyński, Adrian

Kapeller, Marie Lisa

Kapetanakis, Myron

Kapetanović, Nadir

Kapischke, Matthias

Kaplan, Ali Emre

Kapliński, Oleg 
Kaplon, Jan

Kapłonek, Wojciech

Kaplun, Dmitrii

Kapočiūtè-Dzikienė, Jurgita

Kapoutsis, Athanasios

KAPPENSTEIN, Charles

Kapsalis, Vassilis

Kapuscinski, Tomasz

Kapusta, Ireneusz

Kapusta, Łukasz Jan

Kara De Maeijer, Patricia

Karabagias, Ioannis K.

Karabaliev, Miroslav

Karabchevsky, Alina

Karabegovic, Almir

Karabetsos, Efthymios

Karabiyik, Umit

Karagulian, Federico

Karakašić, Mirko

Karakasidis, Theodoros

Karakatič, Sašo

Karakoç, Alp

Karakouzian, Moses

Karaksha, Abdullah

Karalekas, Dimitris

Karalis, Konstantinos

Karalis, Vangelis D.

Karam, Sherif

Karami, Amin

Karampatzakis, Dimitris

Karampidis, Konstantinos

Karampour, Hassan

Karapetis, Stefanos

Karapidakis, Emmanuel

Karaseov, Platon

Karasmanaki, Evangelia

Karatassiou, Maria

Karatkevich, Andrei

Karavai, Olga V.

Karavas, Christos-Spyridon

Karayannis, Chris G.

Karayiannis, Dimitrios

Karayiannis, Nikos

Karbalaei Baba, Alireza

Karbaschian, Ali

Karczemska, Anna

Karczewska, Anna

Karczewski, Jakub

Karczmarczyk, Agnieszka

Kardas, Edyta

Karhunen, Tommi
Kari, Leif

Karim, Aly

Karim, M.

Karim, Md Rajibul

Karimi, Benyamin

Karimian, Nima

Karimi-Rouzbahani, Hamid

Karimov, Artur

Karimullin, Kamil

Karkalos, Nikolaos E.

Karkazis, Panagiotis A.

Karki, Rajesh

Karl, Christian Wolfgang

Karl, Matthias

Karle, Timothy

Karlis, Athanasios

Karlos, Stamatis

Karlovsky, Pavel

Karmakar, Partha

Karnati, Konda Reddy

Karnati, Priyanka

Karnowski, Karol

Karolczuk, Aleksander

Karolinczak, Beata

Karoń, Grzegorz

Karoui, Sofiane

Karouia, Fathi

Karparvarfard, Ali

Karpenko, Anatoly Pavlovach

Karpetis, Adonios

Karpiński, Tomasz M.

Karrech, Ali

Karsch, Stefan

Karsznia, Izabela

Karteris, Emmanouil

Kartheek, Anekella

Kartsonakis, Ioannis

Karunasena, Gami

Karuppannan, Senthil Kumar

Karuppuswami, Saranraj

Karutaa Gnaniar, Sampath Kumar

Karwowski, Krzysztof

Karwowski, Waldemar

Karydas, Andreas

Karyotis, Vasileios

Kasać, Josip

Kasahara, Jun Younes Louhi

Kasai, Naoya

Kašalynas, Irmantas

Kasamatsu, Kenichi

Kasbaoui, Mohamed 
Kashi, Amit

Kasiński, Sławomir

Kaskaoutis, Dimitris

Kasmaee, Sara

Kasparian, Jérôme

Kasperska-Wołowicz, Wiesława

Kasprzak, Artur

Kasprzak, Henryk

Kasprzak, Włodzimierz

Kasprzyk, Jerzy

Kassai, Miklós

Kassal, Petar

Kasser, Michel

Kässi, Tuomo

Kastiukas, Gediminas

Kästner, Christian

Kastner, Wolfgang

Kastrati, Zenun

Kastrisios, Christos

Kastyl, Jaroslav

Kaszuba, Joanna

Katalinic, Marko

Kataoka, Hirokatsu

Kateris, Dimitrios

Katerski, Atanas

Kathiravelu, Pradeeban

Kathuria, Himanshu

Katikou, Panagiota

Katina, Polinpapilinho

Katinić, Marko

Katioglu-Yazan, Tugce

Katira, Parag

Kato, Keisuke

Kato, Shinya

Katogi, Hideaki

Katona, Jozsef

Katrakazas, Christos

Kats, Victor M.

Katsakou, Antigoni

Katsaounidou, Anastasia

Katsavochristou, Anastasia

Katsavounis, Stefanos

Katsigiannis, Yiannis

Katsikas, Sokratis

Katsimpini, Panagiota

Katsoulas, Nikolaos

Kattamuri, Padmanabha V.

Katuchova, Jana

Katula, Levente

Katunin, Andrzej

Katyal, Priya
Katz, Brian F. G.

Katz, Joseph

Katz-Demyanetz, Alexander

Kau, Chung How

Kaufhold, Stephan

Kauhaniemi, Kimmo

Kaul, Sudhir

Kaup, Magdalena

Kaur, Aman

Kaur, Ramanpreet

Kaushik, Abhinav

Kaushik, Abhishek

Kaushik, Neha

Kavadias, Kosmas

Kavčič, Alenka

Kavianpour, Sanaz

Kavoura, Katerina

Kawabata, Norifumi

Kawabata, Yuichiro

Kawai, Toshiyuki

Kawakita, Hidetaka

Kawala-Sterniuk, Aleksandra

Kawalec, Adam

Kawalec, Jacek

Kawalec, Witold

Kawałek, Anna Małgorzata

Kawamura, Izuru

Kawanabe, Hiroshi

Kawano, Jun

Kawata, Shigeo

Kawczyński, Adam

Kawiak, Anna

Kay, Jim W.

Kayser, Klaus

Kayser, Yves

Kazakbaev, Vadim

Kazakovtsev, Lev

Kazama, Motoki

Kazamer, Norbert

Kazanc1, Zafer

Kazanskiy, Nikolay

Kazasidis, Marios E.

Kazda, Tomáš

Kazemi, Ehsan

Kaziev, Andrey V.

Kazimierczak, Renata

Kazimierowicz, Joanna

Kazmi, Jawad

Kazmi, Syed Minhaj Saleem

Kazmierczak, Andrzej R.

Kazuhiro, Kondo 
Kazuro, Momii

Kbashi, Hani J.

Ke, ChihKun

$\mathrm{Ke}$, Tao

$\mathrm{Ke}$, Xie

Kean, Thomas

Kebande, Victor R.

Kechagias, John D.

Kechagias-Stamatis, Odysseas

Kędziora, Anna

Kee, Seong-Hoon

Keech, Pete

Kegalj, Igor

Kehagia, Fotini

Keita, Emmanuel

Kejík, Zdeněk

Kejna, Marek

Kejriwal, Mayank

Keken, Zdeněk

Kelemen, Michal

Keler, Andreas

Keleshteri, M M.

Keller, Dustin

Kelley, Steven

Kelly, James F.

Kelly, Sean

Kelner, Jan

Kemper, Björn

Kendler, Shai

Kennedy, Brook

Kennedy, Eugene T

Kenry, Kenry

Kensek, Karen

Kent, Tom

Kenzari, Samuel

Keoh, Sye Loong

Kephart, Josiah L.

Kępniak, Maja

Keramaris, Evangelos

Keramatikerman, Mahdi

Keramydas, Christos

Kering, Maru K.

Kermani, Mostafa

Kermanidis, Katia Lida

Kern, Frank

Kern, Thorsten A.

Kern, Wolfgang

Kernbach, Julius

Kerner, Boris S.

Kersten, Tobias

Kertész, Gábor
Kesharwani, Siddharth S.

Keshavarzi, Mahmoud

Keshtgar, Azadeh

Kesidis, Anastasios L.

Keskinarkaus, Anja

Kessel, David

Keszöcze, Oliver

Keung Mo, Wai Keung Mo

Khachatryan, Gohar

Khachay, Michael

Khademi, Faezehossadat

Khademi-Vidra, Aniko

Khader, Mahmoud A.

KHADKA, Dhruba B.

Khadka, Roshan

Khajeheian, Datis

Khaki, Saeed

Khalaf, Walaa

Khalaj, Omid

Khaled, Ahmed

Khaled, Yasser

Khaledialidusti, Rasoul

Khaliel, Maher

Khalifa, Amal

Khalifa, Fahmi

Khalili, Fardin

Khalili, Siavash

Khaliq, Jibran

Khalyasmaa, Alexandra

Khambekar, Prashant

Khamis, Mohamed

Khamkhash, Aibyek

Khamukhin, Alexander

Khan, Adil Mehmood

Khan, Arifur R.

Khan, Atif Ali

Khan, Bilal

Khan, Dawar

Khan, Foysal Zahid

Khan, Hamza

Khan, Iftekhar

Khan, Irfan

Khan, Irfan Ahmad

Khan, Md Nazmuzzaman

Khan, Md. Shakhaoath

Khan, Muhammad

Khan, Murad

Khan, Nabeel

Khan, Pervez

Khan, Shahanshah

Khan, Suleman 
Khan, Tanvir

Khan, Usman

Khanal, Mandar

Khanal, Salik Ram

Khandaker, Md. Al-Amin

Khandker, Syed

Khanesari, Mojtaba Ahmadieh

Khanh Le, Nguyen Quoc

Khani, Shaghayegh

Khanna, Sourav

Khanra, Nandish

Khansur, Neamul Hayet

Kharbach, Mourad

Kharel, Rupak

Kharitonov, Dmitry

Khatib, Jamal

Khatib, Tamer

Khatibi, Akbar Afaghi

Khatibi, Seyedalireza

Khatibi, Siamak

Khatir, Samir

Khatoon, Asma

Khattab, Tawfik

Khattak, Shehryar

Khawaja, Hassan

Khazaeinejad, Payam

Kheriji, Sabrine

Khettab, Mohamed

Kheyraddini Mousavi, Behnam

Khezri, Abdolrahman

Khiabani, Vahid

Khlyustova, Anna

Khmaladze, Alexander T.

Khodabakhshnejad, Arman

Khodadadi, Mohammad

Khodadadian, Amirreza

Khojandi, Anahita

Khokhlov, Nikolay

Khokhlov, Valentyn

Kholodnaya, Galina

Kholová, Ivana

Khon, Yu A.

Khonina, Svetlana

Khoramshahi, Ehsan

Khormali, Shahab

Khoshnood, Abbas

Khosravani, Mohammad Reza

Khosravi, Ali

Khosravy, Mahdi

Khosronejad, Ali

Khotinov, Vladislav A.
Khoukhi, Maatouk

Khoury, Zaid

Khozin-Goldberg, Inna

Khraisat, Ansam

Khribi, Lotfi

Khrustalev, Vladislav

Khrustalyov, Anton

Khujanazarov, Temur

Khung, Yit Lung

Khurshid, Chrow

Khusainov, Ramil

Kia, Alalea

Kiam, Jane Jean

Kiani Mavi, Reza

Kiazadeh, Asal

Kibria, B. M. Golam

Kicińska, Alicja

Kickmeier-Rust, Michael

Kida, Małgorzata

Kida, Takuya

Kidando, Emmanuel

Kidron, Giora

Kiel, Jaap

Kielan, Paweł

Kiełbasa, Paweł

Kieliszek, Marek

Kierat, Wojciech

Kiersnowska, Agnieszka

Kierzkowski, Artur

Kiga, Daisuke

Kijek, Arkadiusz

Kijo-Kleczkowska, Agnieszka

Kikhtyanin, Oleg

Kikuchi, Takeshi

Kilanowicz, Anna

Kilby, Jeffrey

Kildashti, Kamyar

Kılıç, Heybet

KILIÇ, Kıvılcım

Kilikevičienė, Kristina

Kilikevičius, Artūras

Kilkovský, Bohuslav

Kilty, Trina J.

Kim, A Ram

Kim, Bong Chul

Kim, Bo-Seung

Kim, Byeong Hee

Kim, Byoungsoo

Kim, Byung-Gyu

Kim, Chang Hun

Kim, Chang Soo 
Kim, Chang-Gi

Kim, Changsoo

Kim, Changwon

Kim, Chan-Jung

Kim, Cheonshik

Kim, Choong Hyun

Kim, Choong-Un

Kim, Chunil

Kim, Chyer

Kim, Daehee

Kim, Dal Hyung

Kim, Deuk-Woo

Kim, Dohoon

Kim, Dong Keun

Kim, Dong Kyu

Kim, Dong-Hyun

Kim, Dong-joo

Kim, Dong-Seong

Kim, Dongwook

Kim, Eui Soo

Kim, Eunwoo

Kim, Gue-Hyun

Kim, Gyeong-Min

Kim, Hae-Won

Kim, Hae-Young

Kim, Hak-Seon

Kim, Harksoo

Kim, Heechul

Kim, Hee-jeong

Kim, HeeSeok

KIM, Heuy Dong

Kim, Hongkook

Kim, Howon

Kim, Hyemin

Kim, Hyeongjoo

Kim, Hyeong-Jun

Kim, Hyun

Kim, Hyun Goo

Kim, Hyun Soo

Kim, Hyung Seok

Kim, Hyung Taek

Kim, Hyungmin

Kim, Hyung-Tae

Kim, Hyunjung

Kim, Hyun-Suk

Kim, Hyun-won

Kim, In-Jung

Kim, Jae Joon

Kim, Jae-Hoon

Kim, Jaeyoon

Kim, Jangho
Kim, Jea Soo

Kim, Jedo

Kim, Jee Taek

KIM, Jeong Soo

Kim, Jeongguk

KIM, Jeong-hwan

Kim, Jibum

Kim, Ji-Hwan

Kim, Jincheol

Kim, Jingyun

Kim, Jinmo

Kim, Jinseok

Kim, Jinwoo

Kim, Jinwook

Kim, Jinyoung

Kim, Jongbok

Kim, Jong-Chan

Kim, Jonghoek

Kim, Jong-Hoon

Kim, Jong-Hwan

Kim, Jongkil

Kim, Jong-Sung

Kim, Joodong

Kim, Joohan

Kim, Joonyoung

Kim, Joseph

Kim, Ju Hyung

Kim, Junbeum

Kim, Jung In

Kim, Jung Kyu

Kim, Jung Tae(Steve)

Kim, Jung Yoon

Kim, Jung-Sik

Kim, Jung-Su

Kim, Jungsuk

Kim, Junmo

Kim, Junseok

Kim, Junsoo

Kim, Juntae

Kim, Kangsoo

Kim, Kee Dae

Kim, Keun Su

Kim, Ki Il

Kim, Kibum

Kim, Ki-Chai

Kim, Ki-Du

Kim, Kihwan

Kim, Ki-Il

Kim, Kiman

Kim, Kwang

Kim, Kwangsoo 
Kim, Kyong Hon

Kim, Kyung Rok

Kim, Kyung-Min

Kim, Kyungsoo

Kim, Kyung-Taek

Kim, Kyuseok

Kim, Min Young

Kim, Min-Soo

Kim, Min-Su

Kim, Min-Suk

Kim, Moonseong

Kim, Myunghoi

Kim, Nam-Gyoon

Kim, Nam-Hoon

Kim, Namjung

Kim, Ok-Su

Kim, Robin

Kim, Samyeon

Kim, Sanghyun

Kim, Sang-Jae

Kim, Sangjin

Kim, Sang-We

Kim, Sang-Young

Kim, Seon-Chil

Kim, Seong Dae

Kim, Seong Su

Kim, Seong-Eun

Kim, Seong-Gon

Kim, Seong-Joon

Kim, Seong-Kyun

Kim, Seoni

Kim, Seung-Joo

Kim, Seungjun

Kim, Seungkeun

Kim, Seungmo

Kim, Shiho

Kim, Sin-young

Kim, Soo

Kim, Soo-Yong

Kim, Sung Jae

Kim, Sung Suk

Kim, Sung-Hee Sonny

Kim, Sungho

Kim, Sung-Hoon

Kim, Sunghyun

Kim, Sungroul

Kim, Sungwan

Kim, Sunjun

Kim, Tae Hyun
Kim, Tae Im

Kim, Taeho

Kim, Tae-Hyong

Kim, Taehyoung

Kim, Tae-Hyun

Kim, Taekyun

Kim, Won Hwa

Kim, Wonho

Kim, Wonjun

Kim, Won-Tae

Kim, Woojin

Kim, Wook-Bae

Kim, Woosuk

Kim, Yong Se

Kim, Yong-Guk

Kim, Yonghee (Korea Advanced Institute of

Science and Technology)

Kim, Yonghee (Korea Conformity

Laboratories)

Kim, Yonghoon

Kim, Yong-Joo

Kim, Yoo-Jun

Kim, Yoon-Chul

Kim, Yoon-Ji

Kim, Young Hoon

Kim, YoungBin

Kim, Youngbok

Kim, Young-Chon

Kim, Young-Gab

Kim, Young-Ju

Kim, Youngmin

Kim, Youngok

Kim, Young-Sik

Kim, Young-Taek

Kim, Youngwoo

Kim, Yountae

Kim, Yunsang

Kim, Yu-Seop

Kimball, Samantha

Kimbaris, Athanasios

Kimiaei, Morteza

Kimoto, Mitsuhiko

Kimovski, Dragi

Kimura, Eizen

Kimuro, Shingo

Kinar, Nicholas J.

Kindinis, Andrea

Kindu, Mengistie

Kindy, Mark S.

King, Scott A.

Kingston, Andrew 
Kinoshita, Hiroyuki

Kinuthia, John

Kinyaevskiy, Igor

Kipouros, Timoleon

Kipyatkova, Irina S.

Kiraga, Marta

Kirane, Mokhtar

Kirchner, Jens

Kirihara, Soshu

Kirker, Grant T.

Kirkham, Sandra

Kirsch, Thorsten

Kiš, Goran

Kis, Viktória Kovács

Kiselev, Vitaly

Kish, Laszlo

Kishcha, Pavel

Kishimoto, Satoshi

Kishimoto, Yo

Kishore, M.B

Kishore, Ratna

Kishore, Vidya

Kišiček, Tomislav

Kisiel, Tomasz

Kisilowski, Jerzy

Kiss, Gabriella B.

Kiss, Imre

Kiss, János

Kiss, Marietta

Kiss, Rita M.

Kissai, Moad

Kistelegdi, István

Kistler, Kurt

Kitagawa, Seiichi

Kitahara, Tatsumi

Kitak, Peter

Kitamura, Yusuke

Kitayama, Fumiya

Kiyono, Ken

Kjeldsen, Eigil

Klačková, Ivana

Klamerus-Iwan, Anna

Klanšek, Uroš

Klapa, Przemysław

Klas, Matej

Klassen, Viktor

Klaus, Jana

Klaus, Katharina

Klebingat, Stefan

Kledrowetz, Vilem

Kleekayai, Thanyaporn
Klein, Axel

Klein, Marcus

Klekiel, Tomasz

Klement, Ivan

KLEMES, JIRI

Klenk, Markus

Klepacka, Joanna

Klepacki, Dariusz

Kletschkowski, Thomas

Klicpera, Milan

Klika, Vaclav

Klimach, Anna

Klimaszewski, Jan

Klimaszyk, Piotr

Klimecka-Tatar, Dorota

Klimek, Katarzyna

Klimek, Radosław

Klimek-Ochab, Magdalena

Klimenda, František

Klimkowicz-Pawlas, Agnieszka

Klimov, Aleksandr

Klisnick, Annie

Kliukas, Romualdas

Kljusurić, Jasenka Gajdoš

Klobučar, Hrvoje

Klobucar, Philip Andrew

Klöckl, Claude

Klomp, Matthijs

Klontzas, Michail

Kłopotowska, Dagmara

Klos, Gunnar

Kłos, Marcin

Klose, Andrew

Klosok-Bazan, Iwona

Kłosowski, Grzegorz

Kłosowski, Łukasz

Kluczyk, Alicja

Kluczyński, Janusz

Klueh, Ulrike

Kluger, Max Oke

Klun, Mateja

Klusman, Ronald W.

Klüss, Joni

Kluza, Krzysztof

Kmet, Stanislav

Kmetič, Ivana

Kmita, Angelika

Kmoch, Alexander

Knápek, Alexandr

Knapek, Michal

Knapic, Sofia 
Knauth, Stefan

Kňazovická, Vladimíra

Knez, Dariusz

Knight, Jason

Kniha, Kristian

Knite, Māris

Knittel, Markus

Knudsen, Erik Bergbäck

Knyazeva, Marina

Knyziak, Piotr

Ko, ByoungChul

Ko, Connie

Ko, Hanseok

Ko, Hyoung Ho

Ko, Jang Myoun

Ko, Kwan-Man

Ko, Yong-Ho

Ko, Young Dae

Kobak, Dmitry

Kobayashi, Atsuko

Kobayashi, Koichi

Kobayashi, Makiko

Kobayashi, Motoyoshi

Kobayashi, Yoshikazu

Koblischka-Veneva, Anjela

Kobrle, Pavel

Kobtsev, Sergey

Kobus, Artur

Kobus, Zbigniew

Kobus-Cisowska, Joanna

Kobylański, Marek

Kobyłecki, Rafał

Kobza, Jozef

Koc, Władysław

Kocáb, Dalibor

Koceva, Aleksandra Rashkovska

Koch, Pierre-Henri

Kochanska, Iwona

Kochetkova (Gudkova), Irina

Kochkin, Pavlo

Kochmańska, Agnieszka

Koci, Kamila

Kocian, Alexander

Kocifaj, Miroslav

Kocijan, Jus

Kociu, Arben

Kociuba, Waldemar

Kocoń, Sławomir

Kocsi, Balázs

Kocsis, Imre

Kocur-Bera, Katarzyna
Koczy, Laszlo T.

Koda, Eugeniusz

Koda, Kazuhisa

Koda, Tomoko

Kodali, Maheedhar

Kodalle, Tim

Kodama, Motoi

Kodet, Jan

Koehler, Bernd

Koelmel, Bernhard

Koenders, E.A.B.

Koenig, Andreas

Koenig, Keith

Koerich, Alessandro Lameiras

Koerner, Stephanie

Koettgen, Julius

Koh, Hyunwook

Koh, Il-Seuk

Koh, Jaehan

Kohan, Rolland

Kohno, Jun-ya

Koike, Kenzo

Koike, Ryo

Koike, Yasuharu

Koike, Yuta

Koit, Mare

Koizumi, Hiroyasu

Koji, Ichii

Kojima, Chie

Kojima, Yoshiyuki

Kokawa, Mito

Kokkalis, Panagiotis

Kokkinos, Evgenios

Kokkinos, Nikolaos

Kokkinos, Panagiotis

Kokol, Peter

Koksharova, Olga A.

Kołakowska, Agata

Kolakowski, Zbigniew

Kolander, Robert

Kolar, Prasanna

Kolar, Vaclav

Kolaric, Branko

Kolarik, Andrew J.

Kolařík, Martin

Kolasa -Wiecek, Alicja

Kolasińska, Ewa

Kolasiński, Piotr

Kolawole, Abimbola

Kolbl Repinc, Sabina

Kölbl, Nathalie 
Kole, Pieter Jan

Kolek, Martin

Kolekova, Yvona

Kolerman, Roni

Koli, Swanand

Kollár, Mojmir

Kollaros, George

Kollmuss, Maximilian

Kolobov, Alexandr Alexandrovich

Kolodziej, Barbara

Kolomvatsos, Kostas

Koltermann, Jan

Koltermann, Jan Jens

Koltsov, Sergei

Kołtunowicz, Tomasz Norbert

Kolyubin, Sergey

Komano, Yuichi

Komar Kawatra, S

Komaris, Dimitrios Sokratis

Komasa, Satoshi

Komeili, Amin

Komęza, Krzysztof

Kommuri, Suneel Kumar

Komninos, Neofytos

Komoda, Ryosuke

Komorek, Andrzej

Komori, Mochimitsu

Komorska, Iwona

Komova, Oxana

Konar, Arkaprabha

Konda Gokuldoss, Prashanth

Kondo, Keiichi

Kondoh, Junji

Kondor, Dániel

Kondratenko, Mikhail S.

Kondratiuk, Mirosław

Konefał, Marek

Konev, Anton

Kong, Kian Hau

Kong, Kyungil

Kong, Pui Wah

Kong, Ran

Kong, Xiangxiong

König, Caroline

König, Ing. Andreas

Konikowska, Beata

Koniorczyk, Marcin

Konkol, Janusz

Könnyü, Balázs

Kono, Daisuke

Kononenko, Oleg V.
Konopacka-Łyskawa, Donata

Konopacki, Maciej

Konorski, Jerzy

Konovalov, Sergey

Konovalov, Сергей Ильич

Konrad, Andreas

Könsgen, Andreas

Konstanciak, Anna

Konstantakopoulos, Ioannis C.

Konstantinidis, Dimitrios

Konstantinidis, Efstathios

Konstantinos, Katakalos

Konstantinova, Marina

Konsulova-Bakalova, Mariya

Kontar, Eduard

Kontargyri, Vassiliki

Kontaxis, Panagiotis

Kontaxis, Panagiotis A.

Kontic, Roman

Kontis, Eleftherios O.

Konto-Ghiorghi, Yoan

Kontogianni, Villy

Kontoleon, Karolos

Kontoni, Denise-Penelope N.

Kontos, Christos K.

Kontziampasis, Dimitrios

Konvalina, Petr

Konys, Agnieszka

Koo, Bon-Yong

Koo, Pyung-Hoi

Koohbor, Behrad

Kooistra, Wiebe H.C.F.

Koolivand, Abdollah

Kopáčik, Alojz

Kopackova, Veronika

Kopanoglu, Emre

Koparan, Cengiz

Kopcakova, Jaroslava

Kopel, Pavel

Koplin, Christof

Kopustinskas, Vytis

Kopustinskiene, Dalia M.

Kopyt, Paweł

Kor, Ah-Lian

Koral, Can

Korbel, Piotr

Kordestani, Mojtaba

Kordopatis-Zilos, Giorgos

Kordos, Damian

Kordoš, Marcel

Kordos, Miroslaw 
Kordov, Krasimir

Koreanschi, Andreea

Koren, David

Koren, Klaus

Korentz, Jacek

Korentzelos, Dimitrios

Korf, Nathalie

Korhonen, Janne

Korka, Zoltan

Korkees, Feras

Korkontzelos, Yannis

Korlos, Apostolos

Korman, Tomislav

Kormanek, Mariusz

Körmann, Fritz

Kornaros, George

Kornguth, Steven E.

Korniejenko, Kinga

Korobeynikov, S.M

Korodi, Adrian

Korolkov, Ilya V

Koronkiewicz, Stanisława

Kortnik, Jože

Korus, Jarosław

Korvel, Grazina

Koryak, Yuri A.

Korytarova, Jana

Korzeniowski, Waldemar

Korzhik, Valery

Korzun, Dmitry

Kosar, Tomaz

Kose, Utku

Kosec, Tadeja

Kosel, Janez

Koseva, Neli

Koshiba, Mamiko

Kosicka-Noworzyń, Katarzyna

Kosiński, Piotr

Kosior, Dominik

Kosir, Rok

Koskela, Markus

Koslowski, Hans Rudolf

Kosman, Wojciech

Kosny, Jan

Kosobudzki, Mariusz

Kosta, Peter

Kostal, David

Kostal, Peter

Kostelecký, Jakub

Kostelić, Katarina

Köstler, Harald
Kostoglou, Margaritis

Kostolný, Igor

Kostopoulos, Georgios

Kostro, Grzegorz

Kostrzewa, Daniel

Kostrzewski, Mariusz

Kostur, Marcin

Kostyła, Paweł

Kostyniuk, Andrii

Kostyuchenko, Evgeny

Koswara, Andy

Koszel, Milan

Koszela, Krzysztof

Koszelew, Jolanta

Koszewnik, Andrzej

Kot, Anna

Kot, Sebastian

Kot, Tomas

Kotcon, James B.

Koteš, Peter

Kothapalli, Sri-Rajasekhar (Raj)

Kotlyar, Victor

Kotobuki, Masashi

Kotov, Leonid

Kotova, Svetlana

Kotowicz, Marek

Kotowski, Tomasz

Kotrasová, Kamila

Kotropoulos, Constantine

Kotsiantis, Sotiris

Kotteda, V M Krushmarao

Kotuliak, Ivan

Kotwica, Krzysztof

Kotyra, Andrzej

Koubassova, Natalia

Koubiti, Mohammed

Koubogiannis, Dimitrios

Koubogiannis, Dimitrios G.

Kouchaki, Samaneh

Koudelková, Zuzana

Koudounas, Konstantinos

Kouketsu, Takeharu

Koul, Sahil

Koulamas, Christos

Koulocheris, Dimitrios

Kouloumpis, Victor

Koumoulis, Dimitrios

Kouris, LAS

Kouritzin, Michael A.

Kourkoumelis, Nikolaos

Kourkoutas, Ioannis 
Kourousis, Kyriakos I.

Kousal, Jaroslav

Kousi, Kalliopi

Koutamanis, Alexander

Koutas, Lampros

Koutelidakis, Antonios E.

Koutelidakis, Ioannis

Koutny, Daniel

Koutras, Athanasios

Koutras, Thanasis

Koutromanos, George

Koutsodendris, Andreas

Koutsouris, Dimitris Dionissios

Koutsovitis, Petros

Kouw, Wouter M.

Kouzes, Richard

Kouziokas, Georgios N.

Kováč, Jaroslav

Kováč, Viliam

Kovacevic, Meho Sasa

Kovacevic, Meho-Saša

Kovacević, Tonko

Kovács, Béla

Kovács, László

Kovács, Tünde

Kovács, Zoltán

Kovács-Öller, Tamás

Kovalenko, Sergey

Kovalev, Anton

Kovalev, Valeri I.

Kovalevsky, Dmitry

Kovalov, Danyil

Kovář, Jiří

Kovari, Attila

Kovářík, Ondřej

Kowalczewski, Przemysław

Kowalczuk, Dorota

Kowalczuk, Zdzisław

Kowalczyk, Marcin

Kowalczyk, Piotr

Kowalczyk, Sebastian

Kowalczyk-Juśko, Alina

Kowalik, Rafał

Kowalska, Beata

Kowalska, Hanna

Kowalska, Jolanta

Kowalska-Góralska, Monika

Kowalska-Koczwara, Alicja

Kowalski, Arkadiusz

Kowalski, Jerzy

Kowalski, Mateusz
Kowalski, Piotr

Kowalski, Radosław

Kowalski, Radoslaw Kajetan

Kowaluk, Grzegorz

Kowsari, Kamran

Koyama, Eiki

Koyanagi, Jun

Kozai, Toyoki

Kozaitis, Samuel

Kozak, Anna

Kozak, Martin

Kozak, Miłosław

Kozakiewicz, Marcin

Kozbur, Damian

Kozel, Tomas

Kozhevnikov, Mikhail

Kozhukhova, Natalia Ivanovna

Kozicki, Marek

Kozieł, Edmund

Kozieł, Sławomir

Kozielski, Lucjan

Kozierkiewicz, Adrianna

Kozik, Andrzej

Koziol, Mateusz

Kozioł, Michał

Kozliak, Evguenii I.

Kozlov, Konstantin

Kozlovskiy, Artem

Kozlowska, Justyna

Kozłowski, Edward

Kozłowski, Marcin

Kozlowski, Michal

Kozuba, Jaroslaw

Kozulovic, Dragan

Kraeima, Joep

Krafft, Frieder

Kraft-Bermuth, Saskia

Kraj, Andrea

Krajcovic, Martin

Krajewski, Marcin

Krajewski, Piotr

Kraker, Elke

Kraleva, Radoslava

Kralevska, Katina

Králik, Juraj

Kralisch, Dana

Kramar, Uroš

Kramer, Phillip

Krammer, Oliver

Kranjčević, Lado

Kranjčić, Josip 
Kranjčić, Nikola

Kranzler, Markus

Krasteva, Vessela

Krasuski, Adam

KRASUSKI, Kamil

Kraszewski, Cezary

Krata, Agnieszka Anna

Kratochvilova, Irena

Kratz, Frédéric

Kratz, Harald

Kratzke, Nane

Krauklis, Andrey E.

Kraus, Armin

Kraus, Jakub

Kraus, Michal

Krause, Andrew

Krause, Hartmut

Krause, Mathias J.

Krauze, Magdalena

Krauze, Piotr

Kravari, Kalliopi

Kravets, Nina

Krawczuk, Marek

Krawczyk, Artur

Krawczyk, Dorota Anna

Krawczyk, Łukasz

Krawiec, Piotr

Krčma, František

Krehula, Ljerka Kratofil

Kreja, Ireneusz

Krejcar, Ondrej

Krejsa, Martin

Kremieniewski, Marcin

Krenicky, Tibor

Krenz, Juliane

Krestenitis, Yannis N.

Kretz, Tobias

Kricka, Tajana

Krishna, Mishra

Krishnamoorthy, Gautham

Krishnan, Girish

Krishnan, Krish

Kristak, Lubos

Kristan, Matej

Kristen, Erwin

Kristian, Yosi

Kristina

Kristjanpoller, Fredy

Kristjánsdóttir, Helga

Kristóf, Tamás

Krisyuk, Vladislav
Kritikakou, Angeliki

Krivoruchko, Alexander

Krivovichev, Vladimir

Krmela, Jan

Krock, Bernd

Kröger, Wolfgang

Krol, Jan

Król, Karol

Król, Małgorzata

Krol, Michał

Królczyk, Grzegorz

Królicka, Agnieszka

Króliczewska, Bożena

Krolikowski, Wieslaw

Kromanis, Rolands

Kronreif, Gernot

Krop, Dave C. J.

Krouska, Akrivi

Krstacic, Goran

Krstanović, Vinko

Krstic, Marija

Krstulović, Nikša

Krstulović-Opara, Lovre

Krtalić, Andrija

Krüger, Björn

Krüger, Marcus

Krüger, Timm

Kruk, Andrzej

Krukiewicz, Katarzyna

Krukowski, Krzysztof

Krūma, Zanda

Krupa, Michał

Krupa-Kozak, Urszula

Krupińska, Izabela

Krupitzer, Christian

Kruszelnicka, Weronika

Kruszyńska, Ewa

Kryjak, Tomasz

Krymskiy, Stanislav

Krysta, Krzysztof

Kryvinska, Natalia

Kryzia, Dominik

Krzaczek, Paweł

Krzemianowski, Zbigniew

Krzesiński, Włodzimierz

Krzton, Ali

Krzysztofik, Izabela

Krzywanski, Jaroslaw

Krzywiecki, Łukasz

Krzywoń, Rafał

Krzywonos, Małgorzata 
Krzyżak, Artur Tadeusz

Krzyżak, Jacek

Krzyzanek, Vladislav

Książek, Marzanna

Ktenidou, Olga-Joan

$\mathrm{Ku}$, Bon-Cheol

$\mathrm{Ku}$, Taeseo

Kuan, Garry

Kuan, Pei-Chen

Kuanar, Shiba

Kubacki, Roman

Kubanek, Mariusz

Kubek, Mario

Kubera, Elżbieta

Kubera, Łukasz

Kubiak, Ireneusz

Kubiak, Katarzyna

Kubica, Jadwiga

Kubicek, Jan

Kubik, Andrzej

Kubík, Lubomír

Kubina, Robert

Kubissa, Wojciech

Kuboki, Takuo

Kubota, Keiichi

Kubota, Shigeki

Kubota, Yoshiki

Kuc, Mateusz

Kučera, Erik

Kučera, Marián

Kučerka, Martin

Kucharczyk, Barbara

Kucharska, Barbara

Kucharska, Edyta

Kucharska, Karolina

Kucharski, Jan

Kuchi, Rambabu

Kucinski, Krzysztof

Kucuk, Israfil

Küçüköz, Betül

Kuczmaszewski, Jozef

Kuczyński, Michał S.

Kudasik, Mateusz

Kudela, Pawel

Kudelcik, Jozef

Kudlaček, Jan

Kudo, Yasuo

Kudra, Grzegorz

Kudreyko, Aleksey

Kudrna, Petr

Kudryashov, Sergey
Kudrynskyi, Zakhar

Kuever, Jan

Kuffer, Monika

Kugler, Szymon

Kuhn, Andreas

Kuht, Jaan

Kuik, Swee

Kujala, Tuomo

Kujan, Omar

Kujawa, Marcin

Kujawska, Justyna

Kukharev, Alexsey

Kukla, Mateusz

Kuksa, Pavel P.

Kukshal, Vandna

Kukula-Koch, Wirginia

Kukulka, David J.

Kukurowski, Norbert

Kula, Krzysztof

Kulan, Mehmet

Kulasinghe, Arutha

Kulawiak, Marek

Kułażyński, Marek

Kulczycka, Joanna

Kulda, Vlastimil

Kulesza, Zbigniew

Kulhandjian, Hovannes

Kulich, Miroslav

Kulicki, Piotr

Kulinich, Sergei

Kulka, Jozef

Kulkarni, Tanmay

Kulpa, Maciej

Kulyukin, Vladimir A.

Kumar, Adarsh

Kumar, Aditya

Kumar, Akhilesh

Kumar, Anuj

Kumar, Avneesh

Kumar, Dushyant

Kumar, Gautam

Kumar, Harish

Kumar, Keshav Senthil

Kumar, Manish

Kumar, Naresh

Kumar, Pandey Prabodh

Kumar, Prakash

Kumar, Samir

Kumar, Sanjay

Kumar, Santosh

Kumar, Shitij 
Kumar, Shivesh

Kumar, Suneel

Kumar, Suren

Kumara, Indika

Kumavor, Patrick D.

Kumchai, Hattanas

Kume, Kensuke

Kun, Ádám

Kunapuli, Gautam

Kundanati, Lakshminath

Kundracik, Frantisek

Kundu, Dipangkar

Kunetsov, Vladimir

Kung, Chung-Wei

Kunkel, Julian

Kunnappilly, Ashalatha

Kunneman, Florian

Kunoh, Tatsuki

Kunova, Andrea

Kuntsevich, Alexander Yu

Kunugi, Tomoaki

Kuo, Chien-Nan

Kuo, Jian-Long

Kuo, Jui-Chao

Kuo, Mao-Kuen

Kuo, Meng-I (Marie)

Kuo, Ming-Tse

Kuo, Po-Chih

Kuo, Sung-Hsin

Kuo, Tsung-Rong

Kuo, Wen-Ten

Kuo, Yong-Lin

Kupecki, Jakub

Kurabayashi, Shuichi

Kuracina, Richard

Kurazume, Ryo

Kurda, Rawaz

Kurdel, Pavol

Kurdi, Abdulaziz

Kurdi, Heba

Kureev, Alexey

Kurek, Andrzej

Kuremoto, Takashi

Kurenda, Andrzej

Kuriakose, Maju

Kurihara, Satoshi

Kurir, Tina Tičinović

Kurita, Hirofumi

Kurkus-Gruszecka, Michalina

Kurniawan, Nicholas A.

Kurnitski, Jarek
Kurpinska, Marzena

Kurt, Mehmet

Kurtser, Polina

Kurumatani, Koichi

Kurushina, Victoria

Kurylo, Piotr

Kurz, Dariusz

Kurz, Marc

Kurzydłowski, Krzysztof J.

Kuś, Wacław

Kusetogullari, Huseyin

Kushkevych, Ivan

Kushner, Alexey

Kushwaha, Pragya

Kusiak, Dariusz

Kusstatscher, Peter

Kustov, Leonid M.

Kustrin, Snezana

Kusuhara, Sentaro

Kusuma, Victor A.

Kusunose, Kenya

Kutafina, Ekaterina

Kutal, Durga H.

Kutasi, Kinga

Kutin, Jože

Kuts, Vladimir

Kutsenko, Anton A.

Kutsyk, Andriy

Kutt, Krzysztof

Kuttner, Christian

Kutylowska, Malgorzata

Kutzner, Rüdiger

Kutzner, Tobias

Kuusk, Kalle

Kuz'min, Mikhail P.

Kuzevič, Štefan

Kuziak, Justyna

Kuzma, Lukasz

Kuźma, Marta

Kuzmin, Alexander

Kuzmin, Leonid

Kuzmina, Ludmila

Kuzmina, Natalia A.

Kuzminykh, Ievgeniia

Kuznetsov, Sergey

Kuznetsov, Yury

Kuznetsova, Iren E.

Kuznetsova, Olga

Kuzuhara, Daiki

Kvasov, Dmitri E.

Kvaternjak, Ivka 
Kvet, Michal

Kvist, Thomas

Kwak, Dongyoup

Kwak, Hyun Duck

Kwak, Hyun Jeong

Kwak, Keun-Chang

Kwame, Amoah B.O.

Kwan, Wingshun

Kwartnik-Pruc, Anita

Kwaśniewska, Anita

Kwek, Leong Chuan

Kwela, Jurek

Kwiatkowski, Mirosław

Kwiatoń, Paweł

Kwiecień, Iwona

Kwiecien, Joanna

Kwinta, Andrzej

Kwoczyńska, Bogusława

Kwok, Kawai

Kwok, Ngai Ming

Kwok, Tsz-Ho

Kwok, Wing-Chi Edmund

Kwon, Dong-Soo

Kwon, Goo-Rak

Kwon, Hyuk-Jun

Kwon, Hyuk-Yoon

Kwon, Hyun

Kwon, Jae-Sung

Kwon, Ki-Chul

Kwon, Ki-Young

Kwon, O. M.

Kwon, Oh Seok

Kwon, Soon-Hong

Kwon, Sunil

Kwon, Tae-Yub

Kwon, You Jin

Kwon, Yu-jin

Kyamakya, Kyandoghere

Kycko, Marlena

Kyei-Baffour, Kwaku

Kylian, Ondřej

Kyoko, Oka

Kyoungsik, Chang

Kyrarini, Maria

Kyratsis, Panagiotis

Kyriakopoulos, Grigorios L.

Kyriakou, Georgios

Kyritsakis, Andreas

Kyun Kim, Do

Kyzas, George Z.

Kyziol, Leslaw
La Foresta, Fabio

La Magna, Antonino

La Rocca, Mario

La Rocca, Michele

La Rocca, Renato V.

La Rosa, Guido

La Rosa, Massimo

La Rosa, Piergiorgio

La Spada, Luigi

La Torraca, Paolo

La, Hung

La, Thanh-Giang

Laarmann, Tim

Labaig-Rueda, Carlos

Labant, Slavomir

Labastida, Ignasi

Labate, Luca

Labella, Alessandro

Labi, Samuel

Labib, S.M.

Labille, Kevin

Labiod, Houda

Labonte, Gilles

Labra Gayo, José Emilio

Labroo, Pratima

LABUN, Ján

Labus, Aleksandra

Labus, Malgorzata

Lacasa, Pilar

Lacasta, Javier

Lăcătușu, Cristina-Mihaela

Lacava, Teodosio

Łach, Michał

Lach, Ralf

Lach, Sławomir

Lache, Simona

Lachman-Senesh, Noa

Lachmayer, Roland

Lachowicz, Marzena

Łaciak, Mariusz

Lacidogna, Giuseppe

Lacitignola, Luca

Lack, Tomas

Lacko, Roman

Lackowski, Marcin

Lada, Zoi G.

Ladosz, Pawel

Ladygin, Anatoly N.

Laefer, Debra

Laefer, Debra F

Lafata, Pavel 
Lafont, Ugo

Lafortune, Stéphane

Lafruit, Gauthier

Laganà, Antonio Simone

Lagarde, Florence

Lagasio, Valentina

Laghi, Vittoria

Lago, Paula

Łagoda, Tadeusz

Lagravère, Manuel $\mathrm{O}$.

Laguarda-Miró, Nicolás

Lagundzin, Dragana

Lahav, Ori

Lahiri, Sriyanka

Lahmiri, Salim

Lahoz, Rafael

Lahoz-Beltra, Rafael

Lai, Chia-Hsiang

Lai, Chian-Hui

Lai, Chiu-Keng

Lai, Chow Yin

Lai, Chun Sing

Lai, Chunkit

Lai, Chunsing

Lai, Cristian

Lai, Derrick Y.F.

Lai, Feipei

Lai, Loi Lei

Lai, Meimei

Lai, Pin-Kuang

Lai, Puxiang

Lai, Qinghua

Lai, Tin Yiu

Lai, Wen-Fu

Lai, Wing-Fu

Lai, Ying-Chih

Laiarinandrasana, Lucien

Laity, Peter R

Laizé, Vincent

Lajeunesse, Dennis Richard

Lajevardipour, Alireza

Lajolo, Carlo

Lajos, Nagy

Lajunen, Antti

Lakatos, Simina

Lake, Jason

Lakshminarayana, Gandham

Lalbakhsh, Ali

Lallart, Mikael

Lallas, Efthymios N.

Lallechere, Sébastien
Laly, Zacharie

Lam, Benson

Lam, Bhan

Lam, Jason

Lam, K.H.

Lamanna, Giuseppe

Lamarche, Larkin

Lamas Galdo, María Isabel

Lamb, David S.

Lamberti, Francesco

Lamberti, Luciano

Lamberti, Patrizia

Lambin, Philippe

Lami, Ihsan

Lamichhane, Anup

Lamichhane, Tej

Lamnawar, Khalid

Lamo, Paula

Lamontagne, Luc

Lamort, Anne-Sophie

Lamouroux, Emmanuel

Lampart, Marek

Lampart, Piotr

Lampert, Cory K.

Lamperti Tornaghi, Marco

Lampreia, Suzana

Lampridi, Maria G.

Lampropoulou, Paraskevi

Lamuta, Caterina

Lamy, Elsa

Lan, Shoufeng

Lança, M. Carmo

Lancaster, Nicholas

Lancea, Camil

Lancellotti, Laura

Landeka Dragičević, Tibela

Landi, Filippo

Landi, Germana

Landi, Luca

Landi, Marco

Landoni, Michela

Landowska, Agnieszka

Landowski, Michał

Lane, Andrew

Lane, Emily

Laner, David

Laneve, Giovanni

Lang, Di

Lang, Nikolaus Wilhelm

Langari, Reza

Langat, Philip Kibet 
Lange, Ewa

Lange, Julia

Lange, Sigrun

Langelaan, David N.

Langemeier, Michael

Langenderfer, Joseph

Langer, Daniel

Langer, Jerzy J

Langer, Miroslav

Langford, Zach

Langowski, Horst-Christian

Lanotte, Ruggero

Lanza, Ezio

Lanza, Jorge

Lanza, Massimo

Lanzarotti, Raffaella

Lanzendörfer, Martin

Lanzi, Massimiliano

Lanzinha, Joao

Lanzolla, Annie

Lanzone, Jacopo

Lao, Seng Kin

Laopoulos, Theodore

Lapaine, Miljenko

Łapczyńska-Kordon, Bogusława

Łapińska, Barbara

Łapiński, Marcin

Łapka, Piotr

Lapkovskis, Vjaceslavs

Lapointe, Mathieu

Lappalainen, Kari

Lappan, Uwe

Lappas, Ilias

Laptev, Alexander

Lapthorn, Andrew Craig

Lara-Cabrera, Raúl

Laribi, Med Amine

Laricchiuta, Annarita

Larijani, Hadi

Larosa, Claudio

Larrañaga, Mikel Mazuela

Larson, Steven R.

Larsson, Mats

Lartigue, Lenaic

Lartillot, Olivier

Larwa, Barbara

Lasalvia, Maria

Lasek, Janusz

Lashkari, Bahman

Lasi, Heiner

Łaskawiec, Edyta
Laski, Pawel Andrzej

Laskowska, Agnieszka

Laskowska, Magdalena

Lassi, Ulla

Lastauskienė, Eglè

Lastra, Miguel

László, Krisztina

László, Makra

László, Vasa

Lata, Michael

Latal, Jan

Latalski, Jarosław

Latella, Christopher

Lates, Mihai-Tiberiu

Latinopoulos, Charilaos

Latorre, M. Pilar

Latorre-Rey, Alvaro D.

Latos-Brozio, Malgorzata

Latosińska, Jolanta

Lattanzio, Susan

Lattuada, Marco

Latychevskaia, Tatiana

Latypov, Rustam Khafizovich

Lau, Michael

Lau, Sam

Lau, Terry Shue Chien

Lau, Yui-yip

Laudani, Antonino

Lauersen, Kyle

Laukkanen, Anne-Maria

Laukkanen, Timo P.

Laupré, Gabriel François

Laurent, Cédric

Laurent, Michaël

Laurenti, Marco

Laureti, Stefano

Laurich, Ben

Laurinavicius, Alfredas

Laurita, Romolo

Lauritano, Dorina

Lavadiya, Dayakar Naik

Lavagna, Luca

Lavdaniti, Maria

Laves, Max-Heinrich

Lavoine, Nathalie

Law, Cheryl Suwen

Law, David

Law, David W.

Law, Yee Wei

Lawman, Samuel

Ławryńczuk, Maciej 
Lazar, Adriana Vulpoi

Lazar, Iwona

Lazar, Josef

Lăzărescu, Adrian-Victor

Lazarescu, Lucian

Lazarescu, Mihai

Lázaro, José

Lázaro, María Teresa

Lazarou, Georgios Y.

Lazarou, Stavros

Lazarov, Andon Dimitrov

Lazarowska, Agnieszka

ŁAŹNIEWSKA-PIEKARCZYK, Beata

Lazopulo, Stanislav

Lazorenko, Georgy

Lazzara, Giuseppe

Lazzaroni, Massimo

Le Bars, Fabrice

Le Bas, Pierre-Yves

Le Bihan, France

Le Coz, Gael

Le Ferrand, Hortense

Le Heron, Richard Bernard

Le Sommer, Nicolas

Le Thanh-Blicharz, Joanna

Le, Anhtuan

Le, Hoa Thanh

Le, Khanh Chau

Le, NamTuan

Le, Nguyen Quoc Khanh

Le, Nguyen-Thinh

Le, Tien Loc

Le, Viet-Duc

Le, Vinh Tung

Lea, Jeremy David

Leach, Felix

Leach, Kyle G.

Leach, Richard

Leal Costa, Cesar

Leal-Junior, Arnaldo

Leary, Edmund

Leba, Monica

Lebedev, Igor

Lebental, Bérengère

Lebiedowicz, Piotr

Lebioda, Marcin

Łebkowski, Andrzej

LEBON, Nicolas

Lèbre, Marie Ange

Lebyodkin, Mikhaïl A.

Leccese, Fabio
Leccese, Francesco

Lech, Piotr

Lechner, Christoph

Lechner, Ulrike

Lecian, Orchidea Maria

Leciejewski, Zbigniew

Lecko, Adam

Ledda, Caterina

Lederer, Ann-Kathrin

Leduc, Damien

Ledvinka, Ondrej

Lee, Bang Yeon

Lee, Byoungho

Lee, ByungKwan

Lee, Changgu

Lee, Changhee

Lee, Changho

Lee, Chang-Hwan

Lee, Chang-Ryeol

Lee, Chang-Soo

Lee, Changyull

Lee, Chi

Lee, Chi Hwan

Lee, Chien-Nan

Lee, Chih-Hao

Lee, Chih-Hsin

Lee, Chul-Hee

Lee, Chul-sung

Lee, Chu-Yu

Lee, Czang-Ho

Lee, Dae-Hyun

Lee, Dai-Soo

Lee, Derher

Lee, Dong Hun

Lee, Dong Myung

Lee, Donggu

Lee, Donghun

Lee, Donghyun

Lee, Dongik

Lee, Dong-Un

Lee, Dong-Yeon

Lee, Doojin

Lee, Du-Hyeong

Lee, Eui Chul

Lee, Gee

Lee, Gee-Soo

Lee, Geon Joon

Lee, Gueesang

Lee, Gyo-woo

Lee, Hansung

Lee, Harry F. 
Lee, Hsiang-Chieh

Lee, Hsin-Yun

Lee, Huang-Chen

Lee, Hyojin

Lee, Hyoung Yong

Lee, Hyung-tae

Lee, Hyunkook

Lee, Hyunseop

Lee, Hyunsoo

Lee, Il-Gu

Lee, In Soo

Lee, Ingyu

Lee, Jae Young

Lee, Jaejun

Lee, Jaesun

Lee, Jaewook

Lee, Jae-yeong

Lee, James W.

Lee, Jangguen

Lee, Jechan

Lee, Jejung

Lee, Jeong Hwan

Lee, Jeong $\mathrm{Su}$

Lee, Jie

Lee, Jihoon

Lee, Jihyun

Lee, Jin Young

Lee, Jin-Ho

Lee, Jin-Shyan

Lee, Jinwoo

Lee, Jinwook

Lee, Jong Yong

Lee, Jong Yun

Lee, Jongbok

Lee, Jong-Ho

Lee, Jonghwan

Lee, Joohyong

Lee, Joonwoo

LEE, JOON-YONG

Lee, Joseph C. Y.

Lee, Ju Han

Lee, Jue-Hyun

Lee, Juhyeong

Lee, Jung Keun

Lee, Jungho

Lee, Jung-Tai

Lee, Jung-Woo

Lee, Ju-Yi

Lee, Kang Taek

Lee, Kee Sung

Lee, Kee-Sun
Lee, Keunhwa

Lee, Kichun

Lee, Kijoon

Lee, Kyoung G.

Lee, Kyoung-Chan

Lee, Kyu-haeng

Lee, Kyungroul

Lee, Kyungwon

Lee, Li-Ang

Lee, Liza

Lee, Manhee

Lee, Maria

Lee, Mark

Lee, Min Cheol

Lee, Moon Gu

Lee, Munjae

Lee, Nam Kon

Lee, O-Joun

Lee, Olive

Lee, Po-Heng (Henry)

Lee, Posen

Lee, Regina

Lee, Sang Gil

Lee, Sang Hyun

Lee, Sang Jun

LEE, Sang Wook

Lee, SangGon

Lee, Sanghwan

Lee, Sang-Kwon

Lee, Sang-Mae

Lee, Sangmin

Lee, Sang-Soo

Lee, Sanguk

Lee, Sang-Woong

Lee, Sean $S$.

Lee, Seokjin

Lee, Seonah

Lee, Seong-Hyeok

Lee, Seongjin

Lee, Seongwook

Lee, Seul-Ki

Lee, Seunghoon

Lee, Seunghwan

LEE, Shu Tak Raymond

Lee, Sihyun

Lee, Soochahn

Lee, Soojeong

Lee, Suan

LEE, SUK JIN

Lee, Sukho

Lee, Sung-Hak 
Lee, Sungju

Lee, Sungon

Lee, Taegyu

Lee, Uen-Do

Lee, Uichin

Lee, Ung-Kyun

Lee, Wei-Chieh

Lee, Wen-Jen

Lee, Winson

Lee, Won

Lee, Woncheol

Lee, Woo Cheol

Lee, Wookjin

Lee, Yen Chien

Lee, Yin-Wen

Lee, Yiu Yin Raymond

Lee, Yoncheol

Lee, Yong Bok

Lee, Yong-cheol

Lee, Yong-Seok

Lee, Young Ji

Lee, Young Ju

Lee, Youngho

Lee, Young-Sil

Lee, Yuh-Ming

Lee, Yun-Ju

Leelőssy, Ádám

Leevy, W. Matthew

Lefebvre, Olivier

Lefik, Marek

Lefkidis, Georg

Lefley, Frank

Leger, Jean-Michel

Leger, Liliane

Legg, Robert

Legrand, Vincent

Legrand, Xavier

Legutko, Stanisław

Lehocky, Marian

Lehuédé, Patrice

Lei, Gang

Lei, Shuting

Lei, Yang

Lei, Yusheng

Lei, Zhou

Leicht, Aleksander

Leichtfuß, Sebastian

Leightley, Daniel

Leineweber, Matthew

Leirós-Rodríguez, Raquel

Leißner, Thomas
Leitão, João

Leitão, Teresa

Leithardt, Valderi

Leiva, Carlos

Leiva, Fabricio

Leiva-Padilla, Paulina

Lejman, Monika

Lekić, Aleksandra

Lekidis, Alexios

Lekidis, Vassilios

Leklou, Nordine

Lelea, Dorin

Leliaert, Jonathan

Leligou, Nelly

Lemanowicz, Marcin

Lembrikov, Boris

Lemiere, Bruno

Lemke, Justyna

Lemma, Enrico

Lemmens, Mathias

Lemmer, Frank

Lemnaru, Camelia

Lemos, José Vieira

Lemu, Hirpa G.

Leń, Przemysław

Lenart, Małgorzata

Lenart, Stanislav

Lenart-Gansiniec, Regina

Leng, Siyang

Lengani, Davide

Lengvarský, Pavol

Lennernas, Hans

Lenstra, Daan

Lentini, Valentina

Lentka, Grzegorz

Lentner, Csaba

Lenton, Gavin

Lenz, Ernst

Lenzo, Basilio

Leo, Marco

Leo, Sabrina

Leon, Francisco

Léonard, Grégoire

Leonardi, Luca

Leonardi, Mauro

Leonardi, Rosalia

Leonardos, Stefanos

Leone, Giovanni

Leong, Ceelia

Leong, Kelvin

Leon-González, Antonio J. 
Leoni, Alfiero

Leonidis, Asterios

Leonow, Sebastian

Leonowicz, Zbigniew

Leonski, Wiesław

Leonzio, Grazia

Leopold, Christian

Leordeanu, Catalin

Lep, Marjan

Lepidi, Marco

Leporini, Mariella

Lerga, Jonatan

Lerman, Dorothea

Lerro, Angelo

Lesar, Boštjan

Lesiuk, Grzegorz

Leśniak, Agnieszka

Leśniewicz, Anna

Leśny, Jacek

Lesselier, Dominique

Lester, Henry

Leszczuk, Agata

Leszczyńska-Sejda, Katarzyna

Leszczyński, Jacek

Leszczyński, Krzysztof

Letcher, Trevor

Leu, Ching-Chich

Leung, Carson

Leung, Colin

Leung, Hui Min

Leung, Mark T.

Leutenegger, Marcel

Leuthold, Juerg

Levacher, Daniel

Levartovsky, Shifra

Levashenko, Vitaly

Levchenko, Igor

Lévêque, Patrick

Lévesque, Benoît

Levesque, Vincent

Levin, Ilya

Levin, Mark

Levin, Mark Sh.

Levin, Oron

Levine, Zachary $\mathrm{H}$.

Levron, Yoash

Lew Yan Voon, Lok

Lewandowicz, Jacek

Lewandowska, Katarzyna

Lewińska, Paulina

Lewiński, Tomasz
Lewis, Leslie C.

Lewis, Phillip

Lewis, Sarah A.

Lexa, Matej

Leylavi Shoushtari, Ali

Lezynski, Piotr

Lezzi, Adriano M.

Lhotska, Lenka

Li, Biao

Li, Bing

Li, Bor-Ran

Li, Boyang

Li, Che-Ming

Li, Cheng

Li, Chengqing

Li, Chengxi

Li, Chenyu

Li, Chih-Ying

Li, Chong

Li, Chunxu

Li, Darson

Li, Dawei

Li, Diao

Li, Dinghua

Li, Feng

Li, Fengqiang

Li, Fuhai

Li, Gang

Li, Guanchen

Li, Guijie

Li, Haijun

Li, Haiyang

Li, Hanchuan

Li, Hao

$\mathrm{Li}, \mathrm{He}$

Li, Heng

Li, Hongjian

Li, Hongyang

Li, Hongyu

Li, Hsing-Hui

Li, Huaizhong

Li, Huiquan

Li, Jia

Li, Jian

Li, Jianghui

Li, Jianing

Li, Jiantong

Li, Jian-wei

$\mathrm{Li}$, Jianxiong

Li, Jianzhao

Li, Jiaqi 


\begin{tabular}{|c|c|}
\hline LI, JICHUN & Li, Xin \\
\hline Li, Jiliang & Li, Xing \\
\hline $\mathrm{Li}$, Jin & Li, Xinlu \\
\hline Li, Jinchao & Li, Xuechao \\
\hline Li, Jinfeng & Li, Yan-cheng \\
\hline Li, Jing & Li, Yang \\
\hline Li, Junhua & Li, Yangmin \\
\hline Li, Kang & Li, Yanlin \\
\hline Li, Kezhi & Li, Yaxin \\
\hline Li, Kuo-Bin & Li, Yeou-Fong \\
\hline Li, Larry K.B. & $\mathrm{Li}, \mathrm{Yi}$ \\
\hline Li, Lengwan & Li, Yi-Chen \\
\hline Li, Lianbo & Li, Yifan \\
\hline Li, Lihua & Li, Yige \\
\hline Li, Lin & Li, Yinglei \\
\hline Li, Linkai & Li, Yuanyuan \\
\hline Li, Liyuan & Li, Yung-Hui \\
\hline Li, Mengfang & Li, Yuping \\
\hline Li, Mengxing & Li, Zhe \\
\hline Li, Miling & Li, Zheling \\
\hline Li, Mingda & Li, Zheming \\
\hline Li, Mingheng & Li, Zhenguo \\
\hline Li, Mo & Li, Zhenming \\
\hline Li, Muzhen & Li, Zhigang \\
\hline Li, Nan & Li, Zhixiong \\
\hline Li, Peng & Li, Zhong \\
\hline Li, Ping & Li, Zushu \\
\hline Li, Qiaochu & Lia, Frederick \\
\hline LI, Qingfeng & Liagre, Bertrand \\
\hline Li, Qiuyi Bing & Liang, Chao \\
\hline Li, Renjie & Liang, Goh Guo \\
\hline Li, Rita Yi Man & Liang, Liang \\
\hline Li, Rujie & Liang, Shunxing \\
\hline Li, Shitao & Liang, Xiao \\
\hline Li, Shuangming & Liang, Xiaolong \\
\hline Li, Shuo & Liang, Xiaoxia \\
\hline Li, Silong & Liang, Zheng \\
\hline Li, Simeng & Liao, Chen-Ting \\
\hline Li, Suyi & Liao, Hsiao-Wei \\
\hline Li, Tao & Liao, Hung-Chang \\
\hline Li, Teng & Liao, Kuo-Wei \\
\hline Li, Tomi T. & Liao, Sam \\
\hline Li, Wei & Liao, Tai-Shan \\
\hline Li, Wei Vivian & Liao, Teh-Lu \\
\hline Li, Weijie & Liao, Xinqin \\
\hline Li, Wenguang & Liao, Yi-Hung \\
\hline Li, Wenjie & Liao, Yuan \\
\hline Li, Xiang & Liau, Ben-Yi \\
\hline Li, Xiaojun & Liaw, Jiun-Jian \\
\hline Li, Xiaopeng & Libera, Justyna \\
\hline Li, Xiaotian & Liberati, Caterina \\
\hline
\end{tabular}




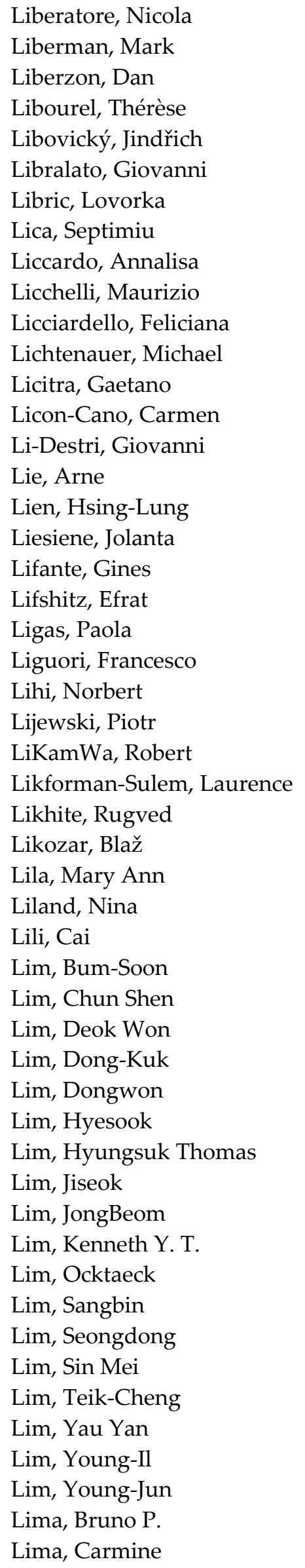

Lima, Ivan

Lima, José Luis

Lima, Sofia

Limiti, Ernesto

Limmer, Steffen

Limongiello, Marco

Limouchi, Elnaz

Lin, Bor-Ren

Lin, Cherng-Yuan

Lin, Che-Wei

Lin, Chien-hong

Lin, Chih-Hong

Lin, Chih-Lang

Lin, Chih-Ping

Lin, Chin-Feng

Lin, Ching Torng

Lin, Chitsan

Lin, Chi-Yi

Lin, Chi-Ying

Lin, Chun-Cheng

Lin, Chun-Liang

Lin, Chun-Ping

Lin, Daw-Tung

Lin, Feng-Cheng

Lin, Gray

Lin, Guan

Lin, Guan-Wei

Lin, Han-Yu

Lin, Hanzhi

Lin, Hong

Lin, Hsin-Hung

Lin, Hsin-Tang

Lin, Hua-Yi

Lin, Huei-Yung

Lin, Jason

Lin, Jerry

Lin, Jonqlan

Lin, Junren

Lin, Ming-Che

Lin, Pao Tai

Lin, San-Shyan

Lin, Shaoyang

Lin, Sheng

Lin, Shih-Chieh

Lin, Shi-Woei

Lin, Tai-Yuan

Lin, Thung-hong

Lin, Ting-Chieh

Lin, Wei

Lin, Wei-Chun

Lin, Weifeng 
Lin, Wei-Ting

Lin, Wen-Yinn

Lin, Will Y.

Lin, Xiao

Lin, Xiaoshan

Lin, Yang

Lin, Yingbo

Lin, Yu-Chen

Lin, Yuh-Yih

Lin, Zhengyu

Lin, Zhibin

Lin, Zihan

Linard, Alexis

Linares-Unamunzaga, Alaitz

Lindeberg, Tony

Linder, Benedikt

Lindner, Arno E.

Lindqvist, Jan Erik

Lindsey-Boltz, Laura

Lindström, Per

Lindström, Tom

Liné, Alain

Linek, Małgorzata

Ling, Alexander

Ling, Bo

Ling, Meng

Linkevičius, Edgaras

Linkous, Clovis A.

Linn, Jimmy B.

Lino, Montoro Moreno

Linß, Sebastian

Linstead, Erik

Linul, Emanoil

Liobikas, Julius

Liobikienè, Genovaitè

Lioe, De Xing

Liotier, Pierre-Jacques

Liou, Ji-Chyuan

Liou, Jr-Jiun

Lipecki, Tomasz

Lipic, Tomislav

Lipiec, Piotr

Lipiński, Seweryn

Lipowczan, Marcin

Liptai, Pavol

Lipuš, Bogdan

Liritzis, Ioannis

Lirkov, Ivan Dimov

Lirn, Ted T. C.

Liro, Maciej

Lis, Andrzej
Lis, Robert

Lisani, José Luis

Lisauskas, Alvydas

Liscak, Pavel

Lisec, Andrej

Lisetskii, Fedor

Lisi, Mariano

Lisi, Matteo

Lisiński, Przemysław

Lisjak, Dragutin

Liška, Anita

Lisnevskaya, Inna

Liso, Vincenzo

Lisowski, Aleksander

Lisowski, Edward

Lisowski, Filip

Lisowski, Józef

Listanti, Marco

Liszkowska, Joanna

Litke, Antonios

Litofsky, Norman Scott

Little, Julian

Litwin, Wojciech

Liu, Bo

Liu, Chao

Liu, Chee Wee

Liu, Cheuk Lun

Liu, Chia-Hui

Liu, Chiang

Liu, Chien-Sheng

Liu, Chih-Chieh

Liu, Ching-Ping Liu

Liu, Damon Shing-Min

Liu, Dan

Liu, Dianzi

Liu, Dongfei

Liu, Filipe

Liu, Gang

Liu, Guangjun

Liu, Guigen

Liu, Hangfan

Liu, Haojie

LIU, Hexu

Liu, Hsiao-Chuan

Liu, Hsien-Kuang

Liu, Huai

Liu, Hu-chen

Liu, Huibin

Liu, Jain-Shing

Liu, Jhy-Chern

Liu, Jianlin 
Liu, Jiechao

Liu, Jih-Shun

Liu, Jingfei

Liu, Jing-Sin

Liu, Jinjie

LIU, Jun

Liu, Jung-Ping

Liu, Kailong

Liu, Kangzhi

Liu, Keng-Ku

Liu, Kouqi

Liu, Kuang-Yen

Liu, Liang

Liu, Linbo

Liu, Maning

Liu, Meng-Kun (Jason)

Liu, Ming

Liu, Ning

Liu, Pei

Liu, Pengfei

Liu, Qi

Liu, Qidi

Liu, Qili

Liu, Rongrong

Liu, Shichao

Liu, Shigang

Liu, Shing-Hong

Liu, Shuanglong

Liu, Shukui

Liu, Tai-Yi

Liu, Teng

Liu, Tingting

Liu, Tsung-Jung

Liu, Tung-Kuan

Liu, Tyng

Liu, Tzong-shi

Liu, Wanchun

Liu, Wei-Cheng

Liu, Weiwei

Liu, Xiao-Zhou

Liu, Xifeng

Liu, Xingjian

Liu, Xiufeng

Liu, Xueyan

Liu, Yan

Liu, Yang

Liu, Yao-Hsien

Liu, Yaolong

Liu, Yiding

Liu, Yi-Hua

Liu, Yiliu
Liu, Yingang

Liu, Yingtao

Liu, Yingyi

Liu, Yonggang

Liu, Yongliang

Liu, Yu

Liu, Yubao

Liu, Yucheng

Liu, Yudong

Liu, YuHao

Liu, Yu-Jen

Liu, Yuming

Liu, Yuqian

Liu, Zeliang

Liu, Zhe

Liu, Zheng

Liu, Ziping

Liu, Ziwen

Liudmila, Radionova

Livens, Stefan

Livreri, Patrizia

Lizcano Casas, David

Lizio, Domenico

Lizio, Giuseppe

Llamas-Nistal, Martín

Llera-Sastresa, Eva

Llorca-Bofí, Josep

Llorens, José Manuel

Llorens-Largo, Faraón

Llorente Isidro, Miguel

Lloret, Jaime

Lloyd, Steven

Lluna, Amparo Gamero

Lo Duca, Angelica

Lo Faro, Maria Josè

Lo Giudice, Angelina

Lo Giudice, Roberto

Lo Iacono, Francesco

Lo Presti, Diego

Lo, Kin Hing

Lo, Kuang Yao

Lo, Shi-Wei

Lo, Wai Lun

Lo, Yu-Sheng

Loas, Gwénolé

Lobato, Ronaldo

Lobo, Paulo Silva

Lobov, Sergey

Locatelli, Marcello

Locatelli, Vittorio

Locci, Antonio Mario 
Lochocki, Benjamin Klaus Peter

Lóczy, Dénes

Loddo, Andrea

Loddo, Donato

Lodewyck, Jérôme

Lodyga-Chruscinska, Elżbieta

Lodygin, Evgeny

Lofrano, Egidio

Logan, Joanne

Logan, Lauren $\mathrm{H}$.

Logan-Sprenger, Heather M.

Logar, Janko

Loglio, Giuseppe

Logo, Janos

Logozzo, Silvia

Loh, Paul

Loh, Ping Yeap

Lohmus, Rynno

Lohne, Jardar

Lohr, Christophe

Loira, Iris

Loja, Maria

Lok Woo, Wai

Lokki, Tapio

Lolli, Nicola

Lollini, Roberto

Lollobrigida, Marco

Lombardi, Angela

Lombardi, Federico

Lombardi, Marco

Lombardi, Tommaso

Lombardo, Salvatore

Lomenie, Nicolas

Lonardo, Amedeo

Lončarić, Ante

Loncoman, Carlos

Londei, Paola

Long, Gavin

Long, Linshuang

Longana, Marco L.

Longe, Omowunmi Mary

Longo, Domenico

Longo, Massimo

Longo, Umile Giuseppe

Longstaffe, James G.

Łoniewski, Igor

Lönnberg, Tuomas

Looi, Kevin

Loose, Maria

Lopatka, Marian

Lopato, Przemyslaw
Lopes Cardoso, Henrique

Lopes, Francisca

Lopes, Marta B.

Lopes, Sergio MR

Lopez Aguirre, Angel Javier

Lopez Anton, Ricardo

Lopez De Heredia, Amaia

López De Lacalle, Luis Norberto

López De Leon, Luis R.

Lopez Elvira, Jose Luis

Lopez Fernandez, Eduardo Jose

Lopez Gayarre, Fernando

López González, Alejandro

López Granado, Otoniel Mario

López Núñez, Juan Antonio

López Ramos, Juan Antonio

López Sánchez, Guillermo Felipe

López, Alicia

López, Concepción

López, Damián

López, Daniel

López, David Marrero

Lopez, Francesco

López, Joaquín

Lopez, Robert

López, Roberto

López, Secundino

López-Chao, Vicente

López-Cortés, Isabel

Lopez-Cuadrado, Jose Luis

López-Espejo, Iván

López-Fernández, Aurelio

López-Fernández, Carlos

López-Fernández, Jorge

López-Fonseca, Rubén

Lopez-Guede, Jose Manuel

López-Gutiérrez, Carlos Javier

Lopez-Jornet, Pia

López-Linares, Karen

López-López, Daniel

Lopez-Molina, Carlos

Lopez-Nuñez, Rafael

López-Ochoa, Luis María

Lopez-Otero, Paula

Lopez-Pellicer, Francisco J.

López-Pintor Muñoz, Rosa María

Lopez-Querol, Susana

López-Sáez, Juan Bosco

López-Salas, Nieves

López-Tirado, Javier

Loprencipe, Giuseppe 
Loprinzi, Paul

Łopusiewicz, Łukasz

Lord, Richard

Lorenc, Augustyn

Lorenzini, Marco

Lorenzini, Matteo

Lorenzo, Antonio Ciruela

Lorenzo, Gonzalo

Lorenzo, Miguel

Loreti, Pierpaolo

Loretz, Brigitta

Loria, Guido Ruggero

Losa Iglesias, Marta Elena

Losada Gutiérrez, Cristina

Losada, M. Ángeles

Losanno, Daniele

Loster, Jolanta Elżbieta

Lotfian, Saeid

Lott, Dieter

Lou, Der-Chyuan

Lou, Shuai

Louda, Petr

Loughry, Joe

Louis, Joseph

Louis, Julien

Louka, Panagiota

Loukatos, Dimitrios

Loupa, Glykeria

Loureiro, Nuno Calçada

Lourenço, João

Louwen, Atse

Lovasz, Erwin-Christian

Love, J. Shayne

Loveridge, Melanie

Lovric, Dino

Loya, J.A.

Löytönen, Markku

Lozac'h, Mickaël

Lozano Guerrero, Antonio José

Lozano Murciego, Alvaro

Lozano, Elisa

Lozano-Galant, Jose Antonio

Lozinca, Eugen

Lozito, Gabriele Maria

$\mathrm{Lu}$, Chao

Lu, Chia-Liang

Lu, Chien-Yu

Lu, Chih-Heng

Lu, Ching-Ta

Lu, Chun-Ping

$\mathrm{Lu}$, Fei
$\mathrm{Lu}$, Guoxing

Lu, Huitian

Lu, Jiang

$\mathrm{Lu}$, Jinzhi

Lu, John C.-C.

Lu, Kuo-Chang

$\mathrm{Lu}, \mathrm{Li}$

Lu, Liqiang

$\mathrm{Lu}$, Meng

Lu, Mingyang

Lu, Mingyu

Lu, Rongxing

Lu, Ruochen

Lu, Weizhuo

Lu, Xingxu

$\mathrm{Lu}$, Yang

Lu, Yiji

Lu, Yubing

LU, Yuzhen

Lu, Zichen

Lubatsch, Andreas

Luboš, Smolík

Luc, Jérome

Luca, Andrei

Luca, Dorin

Lucà, Federica

Lucarelli, Caterina

Lucas, Donald

Lucena, Rafael

Luchinat, Claudio

Lucia, Umberto

Luciani, Giuseppina

Lučić, Bono

Lucklum, Frieder

Luckner, Marcin

Lucyshyn, Thomas

Luczak, Tony

Ludkowski, Sergey

Ludovico Marques, Marco António

Ludowicz, Wojciech

Ludwig, Ralf

Luebken, Tilo

Luedeling, Eike

Luesma, María José

Luh, Jer-Junn

Lühr, Carsten

Lui, Antonny

Lui, Eric

Luin, Blaž

Luino, Fabio

Luis, Ana 
Luis-Alfonso

Luiza Pop, Izabela

Luján-Mora, Sergio

Lukač, Zrinka

Lukačević, Ivan

Łukasz Jan, Kapusta

Lukasz, Wycislik

Łukaszewicz, Andrzej

Lukichev, Dmitry

Lukinac Čačić, Jasmina

Lukitawesa, Lukitawesa

Lukiw, Walter

Lukomska-Szymanska, Monika

Lukomski, Michal

Lukoševičienè, Ona

Lukoševičius, Saulius

Lukoyanov, Alexey

Lukyanenko, Dmitry

Lukyanov, Pavel A.

Lumentut, Mikail

Luna Maldonado, Aurelio

Luna, Carlos

Luna, Diego

Luna, José María

Luna-Perejón, Francisco

Lund, Anja

Lundgren, Lina Lundgren

Lung, Ming-Yu

Lungu, Adrian

Lungu, Magdalena Valentina

Lunguleasa, Aurel

Lunkov, Andrey

Lunt, Alexander

Luo, Chunbo

Luo, Jia-Ning

Luo, Longxi

Luo, Ningqi

Luo, Shaocheng

Luo, Win-jet

Luo, Xian

Luo, Xichun

Luo, Yanhua

Luong, Minh

Luongo, Angelo

Lupi, Saturnino Marco

Lupiano, Valeria

Lupo, Cosmo

Lupu, Ciprian

Lupu, Dan

Lupu, Eugen

Lupulescu, Marian V.
Luqman, Muhammad Muzzamil

Luque Vega, Luis Fernando

Luque, Amalia

Luque, Gabriel

Luque-Nieto, Miguel Angel

Lura, Derek

Łusakowski, Jerzy

Lussier, Benjamin

Lüth, Stefan

Lützenkirchen-Hecht, Dirk

Luukkonen, Tero

Lv, Jinglei

Ly, Ramatou

Lydon, Myra

Lyhne, Mads Dam

Lykidis, Charalampos

Lymperakis, Liverios

Lynam, Mary

Lynch, Doug

Lyons, Ashley

Lyridis, Dimitrios V.

Lysenko, Sergiy

Łysiak, Grzegorz

Lyu, Fanchao

Lyu, Hailong

Lyu, Hongming

Lyubimova, Tatyana P.

Lyubushin, Alexey

M'Koma, Amosy

M'zoughi, Fares

Ma, Chih-Ming

Ma, Fa-Jun

Ma, Guancong

Ma, Hongjie

Ma, Hongyang

Ma, Jia

Ma, Kesen

Ma, Ming

Ma, Qianli

MA, QINGLANG

Ma, Qingqing

Ma, Shang-Pin

Ma, Shuangge

Ma, Tai-Yu

Ma, Tao

Ma, Tong

Ma, Wenxiu

Ma, Xing

Ma, Xingmao

Ma, Yan

Ma, Yongsheng 
Maak, Steffen

Maalouf, Chadi

Maaref, Hichem

Maarouf, Ahmed

Maas, Jürgen

Maass, Anna-Lisa

Mabbott, Samuel

Mabrouk, Rostom

Mabuchi, Ryota

Macas, Martin

Macauley, Matthew S.

MacBean, Victoria

Maccaferri, Nicolò

Maccagno, Massimo

Macdonald, Digby

Macdougall, Colin

Macedo, Maria Filomena

Macek, Jan

Macenić, Marija

Maćešić, Dubravko

Mach, Łukasz

Mach, Mojmir

Machacek, Joshua

Machacek, Zdenek

Machado, José

Machaj, Juraj

Machicoane, Nathanael

Machikhin, Alexander

Machno, Magdalena

Machrafi, Hatim

Machura, Lukasz

Macia, Llorenc

Maciąg, Rafał

Macías, Elsa M.

Macias-Gonzalez, Manuel

Maciejczyk, Polina

Maciejewski, Grzegorz

Maciejewski, Henryk

Macioszek, Elżbieta

Maciuk, Kamil

MacKenzie, Kenneth J.D.

MacKerell, Alexander

Mackiewicz, Piotr

MACKO, Marek

MacNeil, Mike

Macor, Alarico

Macq, Benoit

Macvicar, Bruce

Macwan, Isaac

Mączka, Mirosław

Madalina, Iuga
Madamopoulos, Nicholas

Madani, Abbas

Madbouly, Samy

Maddalena, Lorenza

Maddi, Abhiram

Maddio, Pietro Davide

Madeira, Filipe

Madeira, Rui Neves

Madej, Dawid

Madej, Dominika

Madejón, Paula

Mademli, Lida

Madison, Alexey E.

Madokoro, Hirokazu

Madruga, Santiago

Madsen, Dag Øivind

Madudova, Emilia

Madueño, Leizel

Maekawa, Koichi

Maestre Vidal, Jorge

Maestre, Rafael

Maestrelli, Piero

Maffei, Gianluca

Maga, Daniel

Maga, Dušan

Magalhães, Ricardo

Magaña Redondo, Pedro J.

Magán-Carrión, Roberto

Magan-Fernandez, Antonio

Magaril, Elena

Magdziak, Marek

Maggos, Thomas

Magier, Mariusz

Magklara, Angeliki

Magkos, Emmanouil

Maglaveras, Nicos

Maglić, Livia

Magni, Mirko

Magnone, Daniel

Magoulas, George

Magraner, Teresa

Magreñán, Ángel Alberto

Magris, Martin

Magro, Cátia

Maguire, Marc

Maguire, Martin

Magyar, Attila

Mahabadipour, Hamidreza

Mahabaduge, Hasitha

Mahadik, Bhushan

Mahajan, Harshal 
Mahan, J. Robert

Mahapatra, Manoj K.

Mahar, Rohit

Mahbub, Upal

Mahdavi, Mahboobe

Mahdi, Samira

Mahedi, Masrur

Mahian, Omid

Mahjouri-Samani, Masoud

Mahmood, Haroon

Mahmoud, Ahmed

Mahmoud, Qusay H.

Mahnič-Kalamiza, Samo

Mahon, Peter J.

Mahovic Poljaček, Sanja

Mahy, Julien

Maia, Cláudio

Maia, Lino

Maia, Rodrigo Filev

Maiello, Guido

Maietti, Federica

Maigha, Maigha

Maihami, Reza

Maimaitiyiming, Matthew

Maina, Martin Ruthandi

Mainardis, Matia

Maingi, John

Mainprize, James

Maiorana, Carlo

Maire, Frederic

Maisch, Tim

Maiti, George

Maître, Julien

Maity, Debotyam

Maiuolo, Loredana

Maj, Michał

Maj, Piotr

Majak, Jüri

Majchrowicz, Daria

Majdański, Mariusz

Majeed, Abdul

Majeed, Farhat

Majer, Anna

Majewska, Katarzyna

Majewski, Artur J

Majewski, Jacek A.

Majewski, Maciej

Majidi Nezhad, Meysam

Majka, Andrzej

Majkowska-Marzec, Beata

Majorana, Carmelo
Majumdar, Gourab

Majumder, Sudip

Maj-Zurawska, Magdalena

MAK, Hugo Wai Leung

Makantasis, Konstantinos

Makarov, Dmitriy V.

Makarov, Igor

Makarov, Sergey

Makarova, Katerina

Mäkelä, Ville

Makhmalbaf, Atefe

Maki, Teruya

Mäki-Arvela, Päivi

Makihara, Kanjuro

Mäkinen, Sari

Makita, Kohei

Maknickas, Algirdas

Makoś, Patrycja

Makovická Osvaldová, Linda

Makowska, Malgorazata

Makowski, Łukasz

Makowski, Marcin

Makowski, Michał

Makowski, Nathan

Makris, Christos

Makris, Sotiris

Makrygiorgou, Despoina

Maksimov, Igor V.

Maktabi, Marianne

Malachowski, Jerzy

Malagon, Pedro

Malaiškienè, Jurgita

Malajner, Marko

Malallah, Ra'ed

Malandrino, Mery

Malara, Jarosław

Malassiotis, Sotiris

Małaszkiewicz, Dorota

Malatesta, Gianluca

Malça, João

Malchik, Fyodor

Malchiodi, Dario

Maldonado Hodar, Francisco José

Maldonado, Saturnino

Maléchaux, Astrid

Malecka, Joanna

Malek Mohammadi, Mohammadreza

Málek, Jaroslav

Małek, Marcin

Malekan, Mohammad

Malekjafarian, Abdollah 
Malek-Madani, Reza

Malekmohammadi, Hamed

Malenica, Luka

Malesza, Wiktor

Malet, Loïc Charles

Maletti, Sebastian

Malhotra, Deepika

Malik, Amir Haider

Malik, Sharali

Malikan, Mohammad

Malinas, Anamaria

Malinen, Mikko

Malinovskaya, Svetlana

Malinowski, Paweł $\mathrm{H}$.

Malkin, Alexander

Malkowski, Piotr

Malladi, Sriram

Mallen, Evan

Mallikarachchi, Thanuja

Mallinis, Giorgios

Mallio, Carlo Augusto

Malomo, Daniele

Malov, Alexander I.

Maltar-Strmečki, Nadica

Maltman, Chris

Maltry, Laura

Maluenda, David

Malvè, Mauro

Malvić, Tomislav

Malvic, Tomislov

Malvoni, Maria

Maly, Kurt

Malykh, Sergey

Malyuskin, Oleksandr

Mamaev, Ivan Sergeevich

Mambretti, Stefano

Mamede, Jorge

Mamì, Antonella

Mamiya, Ping C.

Mammola, Caterina Loredana

Mamrashev, Alexander

Man Li, Rita Yi

Man, Adrian

Man, Siu Shing

Manabu, SHIBASAKI

Manalil Velayudhan, Sudheesh

Mañana, Mario

Manara, Giuliano

Manariotis, Ioannis D.

Maňas, Pavel

Manca, Oronzio
Manca, Stefania

Mance, Davor

Mance, Diana

Mancini, Marco

Mancini, Simone

Mancino, Luca

Mancisidor, Aitziber

Mancuso, Antonio

Mancuso, Megan E.

Mandal, Subhamoy

Mandatori, Domitilla

Mandelli, Stefano

Mandičák, Tomáš

Mandl, Thomas

MANDOLESI, SERENA

Mandolini, Marco

Mandracci, Pietro

Mandric, Igor

Mandrioli, Riccardo

Mandžuka, Sadko

Manea, Avram

Manetto, Giuseppe Ezio

Manfredi, Luigi

Mangalathu, Sujith

Mangiameli, Michele

Mangini, Agostino Marcello

Manheim, Derek

Mania, Szymon

Manica, Rogerio

Manica, Scheila

Manich, Salvador

Manicone, Paolo Francesco

Manière, Charles

Maniglia, Bianca

Manika, Georgia

Manika, Stella

Manikas, Theodore W.

Manis, George

Mankey, Gary

Mankin, Richard W.

Mankovits, Tamás

Mann, Francis

Mann, Steve

Männer, Jörg

Manneville, Paul

Mannheim, Viktoria

Manno, Vincent

Mannocci, Andrea

Mannodi Kanakkithodi, Arun Kumar

Mannu, Alberto

Mano, Hiroki 
Manogaran, Gunasekaran

Manojlović, Vaso

Manos, George C.

Manosalvas-Paredes, Mario

Manowska, Anna

Manrique Rosel, Javier

Mansell, J.P.

Manso, Pedro

Manson, Neil

Mansour, Gabriel

Mansour, Omar

Mansour, Rabih

Mansouri, Abraham

Mansouri, Shahnaz

Månsson, Sven

Manta, Ioana

Manta, Vasile

Mántaras, Daniel Álvarez

Mantawy, Islam M.

Mante, Francis K.

Mantell, Casimiro

Mantione, Daniele

Mantzaris, Alexander V.

Mantzourani, Ioanna

Mantzouridou, Fani

Manuel Vieira Pombo, António

Manuel, Fernández López-Vizcaíno

Manumachu, Ravi Reddy

Manuri, Federico

Manwar, Rayyan

Manwell, James

Manyk, Tetiana

Manzalini, Antonio

Manzanares-Lopez, Pilar

Manzanera, Antoine

Manzo, Mario

Manzo, Maurizio

Manzoni, Cristian

Manzoni, Stefano

Manzur, Tanvir

Mao, Chengsheng

Mao, Gaojun

Mao, Lei

Mao, Yijie (Lina)

Mar, Kathleen

Marachevsky, Valery

Maradin, Mladen

Maraffi, Sabina

Maraloiu, Valentin-Adrian

Maranan, Marlon

Maranesi, Margherita
Maranesi, Monica

Marani, Pietro

Marani, Roberto

Marapulets, Yury

Marasco, Daniela

Marasová, Daniela

Marasović, Tea

Marasso, Simone Luigi

Marateb, Hamid Reza

Maraveas, Chrysanthos

Marazuela, Miguel Ángel

Marc, Courte

Marc, Gabriel

Marcalikova, Zuzana

Marceau, François

Marcel, Müller

Marcellini, Moreno

Marchelli, Filippo

Marchenkov, Artem

Marchesoni, Mario

Marchetti, Claudio

Marchetti, Francesca

Marchi, Beatrice

Marchi, Luca

Marchionni, Andrea

Marchisio, Alberto

Marchuk, Andrey

Marchuk, Oleksandr

Marcian, Petr

Marcin, Wardach

Marciniak, Zbigniew

Marciniak-Lukasiak, Katarzyna

Marčiš, Marian

Marco, Bietresato

Marco, Felipe King

Marco, Hector

Marcon, Petr

Marconcini, Paolo

Marconi, Marco

Marcos, Marcos

Marco-Such, Manuel

Marcu, Ioana

Marcuccio, Salvo

Marcus, Ingram

Marczewska, Barbara

Marczewski, Jacek

Mardani, Abbas

Mardani, Hossein

Maréchal, Manuel

Marek, Aleksander

Marek, Kieliszek 
Mares, Jan

Mareschi, Katia

Margara, Alessandro

Mărgărint, Mihai Ciprian

Margaritis, Lukas

Margaritondo, Giorgio

Margherita, Emanuele Gabriel

Marghitu, Dan

Mărginean, Gabriela

Margiotta, Azzurra

María Del Molero-Jurado, Maria

Maria Musarella, Carmelo

Maria Simona, Raboaca

María, Soria-Oliver

Marian, Dacian-Paul

Marian, Max

Marian, Romeo

Mariana, Iorgulescu

Mariani, Guido Stefano

Mariani, Stefano (Politecnico di Milano)

Mariani, Stefano (University of Modena and Reggio Emilia)

Mariano, Stefania

Mariasiu, Florin

Marić, Predrag

Mariello, Massimo

Marie-Thérèse

Marietta, Fodor

Mariggiò, Maria A.

Marin, Luminita

Marin, Mariana

Marina, Krvavica

Marinakis, Vangelis

Marinelli, Mario

Marinello, Francesco

Marinescu, Maria

Marini, Federico

Marini, Martino

Marini, Mauro

Mariniello, Loredana

Marín-Marín, José-Antonio

Marino, Michele

Marín-Sáez, Julia

Marinucci, Lorella

Marinus, Benoit

Mario, Pagnoni

Mariscotti, Andrea

Marita, Tiberiu

Marius, Bodor

Mariusz, Domagala

Markdahl, Johan
Markelov, Oleg A.

Marken, Frank

Markéta, Miháliková

Markiewicz, Aleksandra

Marklund, Marie

Marko, Matthew

Markopoulos, Angelos P.

Markötter, Henning

Markou, Giorgos

Markov, Krassimir

Marková, Iveta

Marković, Monika

Markowicz-Piasecka, Magdalena

Markowski, Piotr

Markušić, Snježana

Markvicka, Eric

Marletta, Luisa

Marling, Garry

Marmo, Rossella

Marmy, Pierre

Maroteaux, Luc

Maroto-Molina, Francisco

Marović, Ivan

Marques, Carlos

Marques, Duarte

Marques, Fernando

Marques, Filipe

Marques, Gonçalo

Marques, Manuel Joaquim Bastos

Marques, Marco Ludovico

Marques, Viriato

Marquez Alcaide, Abraham

Marquez Ruiz, Alejandro

Marquez, Carlos

Márquez, Fausto Pedro García

Marrale, Maurizio

Marrasso, Elisa

Marrazzo, Pasquale

Marrelli, Massimo

Marrero, Domingo

Marrocco, Valeria

Marrone, Stefano

MARS, Kamel

Marsano, Davide

Marseglia, Guido

Marseille, Gert-Jan

Marshal, Jolanta

Marshall, Andrew G.

Marsico, Antonio Domenico

Marsili, Stefania 
Mart'1nez-del-Amor, Miguel A.

Marta, Ruiz Llata

Martalò, Marco

Marteau, Philippe

Martel, Ernestina

Martelli, Francesco

Martens, William

Martian, Alexandru

Marticorena-Sánchez, Raúl

Martikka, Ossi

Martín Álvaro, Conchita

Martín Dorta, Norena

Martín San Agustín, Rodrigo

Martin, Dan

Martín, David Orellana

Martin, Eric

Martin, Iain

Martin, Jessica

Martín, Joan Navarro

Martín, Manuel Jesús Cobo

Martin, Maria-Jesus

Martin, Philip

Martin, Vicente

Martina, Matteo

Martin-Candilejo, Araceli

Martinčić-Ipšić, Sanda

Martinell, Julio

Martinelli, Alessio

Martinelli, Enzo

Martinelli, Massimo

Martines, Emilio

Martinetti, Alberto

Martínez Díaz, José J.

Martínez Gila, Diego Manuel

Martinez Gonzalez, Jose Angel

Martínez Guardiola, Francisco Javier

Martínez López, Luis

Martínez Rodrigo, Javier

Martinez Rojas, Juan Antonio

Martínez Segura, Marcos Antonio

Martínez Torres, Javier

Martínez, Emilio Pascual

Martínez, Félix Quintero

Martínez, Francisco Guijarro

Martínez, José Luis

Martinez, Josu Etxezarreta

Martinez, Manuel

Martínez, Pedro Juan

Martínez-Álvarez, Francisco

Martínez-Ávila, Daniel

Martínez-Carricondo, Patricio
Martinez-de-Rioja, Eduardo

Martínez-Echevarría Romero, María José

Martínez-Espinosa, Rosa María

Martínez-Ferrer, Pedro J.

Martínez-García, Herminio

Martínez-García, Rebeca

Martínez-González-Moro, Ignacio

Martínez-Guardado, Ismael

Martínez-Hinarejos, Carlos-D.

Martínez-Márquez, Camilo I.

Martinez-Martin, Ester

Martínez-Martínez, Víctor

Martínez-Otzeta, José María

Martínez-Pañeda, Emilio

Martínez-Rocamora, Alejandro

Martinez-Rojas, Enriqueta

Martínez-Rojas, María

Martínez-Sánchez, Gregorio

Martínez-Sanz, José Miguel

Martínez-Tejada, Laura Alejandra

Martin-Fernandez, Cristian

Martín-Fernández, Susana

Martin-Gutierrez, Jorge

Martín-Hidalgo, David

Martinho, Vítor João Pereira Domingues

Martini, Alberto

Martini, Elisabetta

Martiniano, Hugo

Martinka, Jozef

Martinkovic, Maros

Martín-Lammerding, Diana

Martín-Martín, Pilar

Martino, Francesco

Martino, Luca

Martino, Sabata

Martinotti, Simona

Martín-Ramos, Juan A.

Martín-Rapún, Rafael

Martin-Rodilla, Patricia

Martins Gomes, João Manuel

Martins, Alberto M. B.

Martins, Ana Maria Tavares

Martins, André Dias

Martins, Felipe Nascimento

Martins, Fernando M. L.

Martins, Marcos Silva

Martins, Rafael Messias

Martins, Rui F.

Martis, Claudia

Marto, Carlos Miguel

Márton, Kivovics 
Márton, Lorinc

Marton, Peter

Martone, Alfonso

Martos Martínez, África

Martsenyuk, Vasyl

Martucciello, Nadia

Martynowicz, Helena

Martynowicz, Pawel

Marucci, Alvaro

Maruccio, Giuseppe

Maruda, Radosław W.

Marulanda, Geovanny

Marušić, Katarina

Maruthamuthu, Murali

Maruyama, Yoshihisa

Maryska, Milos

Marzantowicz, Łukasz

Marzec, Michał

Marzec-Grządziel, Anna

Marzeddu, Simone

Marzi, Claudia

Marzo, Leyre

Marzocca, Cristoforo

Marzola, Antonio

Masago, Yoshifumi

Masamune, Sadao

Masarati, Pierangelo

Masashi, Ishikawa

Mas-Bargues, Cristina

Mascaretti, Luca

Mascher-Frutschi, Fabio

Mascherini, Gabriele

Masci, Paolo

Mascio, Tania Di

Mascort-Albea, Emilio J.

Masdías-Bonome, Antonio

Masek, Jiri

Masera, Gabriele

Mashaghi, Alireza

Mashima, Daisuke

Mashita, Tomohiro

Masiero, Andrea

Maskeliunas, Rytis

Maskeliūnas, Ryts

Maskey, Mahesh Lal

Maskil-Leitan, Reuven

Maskooni, Ehsan Kamali

Masłoń, Adam

Maslov, Andrey V.

Maslov, Dmitry A.

Maśnicki, Romuald
Masoero, Marco

Masone, Adriano

Masood, Nahid Al

Masoodi, Amirreza

Masoud, Mahmoud

Masoudi Soltani, Salman

Maspero, Cinzia

Maspero, Federico

Massa, Nadia

Massari, Francesco

Massaro, Matteo

Massaro, Sebastiano

Massarotti, Nicola

Massera, Jonathan

Massidda, Luca

Massier, Tobias

Massimo Foglia, Mario

Massot-Campos, Miquel

Mastalska-Popławska, Joanna

Mastanjević, Kristina

Mastelić Ivić, Siniša

Mastellone, Maria Laura

Mastinu, Giampiero

Mastmeyer, Andre

Mastropietro, Alfonso

Mastrotheodoros, Georgios P.

Mastrucci, Alessio

Masubuchi, Yuji

Masuda, Shumpei

Masuda, Yoichi

Masullo, Alessandro

Masum, Shakil

Masumoto, Hidetoshi

Masurkar, Nirul

Mata, Luis A.

Mata-Carballeira, Óscar

Matache, Mihai Gabriel

Mata-Lima, Herlander

Matarese, Giovanni

Mataric, Maja J.

Matas, José

Matasaru, Petre Daniel

Matassoni, Marco

Mateescu, Carmen

Mateev, Valentin

Matei, Alina

Matejec, Vlastimil

Mateo, Carlos M.

Mateo-Garcia, Monica

Mateos, Cristian

Materazzi, Massimiliano 
Materka, Andrzej

Materska, Małgorzata

Matetić, Maja

Mateus, Dina M. R.

Mateus, Luís

Mateus, Ricardo

Mathan, Bobby

Mathelin, Lionel

Mathew, Mona

Mathias, Jean-Denis

Matias, João

Mati-Baouche, Narimane

Matić, Tomislav

Matichenkov, Vladimir V.

Matiu, Michael

Mativenga, Mallory

Matko, Vojko

Matkowski, Adam

Matłok, Natalia

Matos, Inês

Matos, João Nuno

Matos, Sergio

Matosas-López, Luis

Matran-Fernandez, Ana

Matranga, Domenica

Matras-Postolek, Katarzyna

Matras-Postołek, Katarzyna

Matsakidou, Anthia

Matsiola, Maria

Matsuda, Takashi

Matsuda, Yukiko

Matsugaki, Aira

Matsugi, Akiyoshi

Matsuhisa, Fumikazu

Matsuhisa, Naoji

Matsukura, Haruka

MATSUMOTO, Arimasa

Matsumoto, Hiroshi

Matsumoto, Yukihiro

Matsumura, Kazuaki

Matsuo, Tatsuhito

Matsushita, Kensuke

Matsuzaka, Yasunari

Matsuzaki, Takashi

Mattei, Francesca

Matteo, Laura

Matteo, Vannini

Matteucci, Enrica

Matteucci, Francesco

Mattia, Barbarino

Mattoli, Virgilio
Mattoni, Benedetta

Matttheakis, Marios

Matul, Alexander

Matulic, Maja

Matulin, Marko

Matusiewicz, Maria

Matušů, Radek

Matuszewski, Jan

Matuszka, Tamás

Matwijczuk, Arkadiusz

Matys, Jacek

Matyska, Vojtěch

Matz, Radosław

Matzner, Shari

Maudes, Jesus

Maugeri, Grazia

Mauri, Roberto

Mauriello, Filomena

Mauritzen, Jesper Juel

Mauro, Francesco

Mauro, Giorgio Sebastiano

Mauro, Giovanni

Mauro, Noemi

Mauro, Stefano

Mauro, Vasconi

Maus, Sönke

Mauša, Goran

Mavragani, Amaryllis

Mavroidis, Iakovos

Mavromatis, Ioannis

Mavropoulos, Georgios

Mavros, George

Maxim, Anca

Maximov, Ivan

Maximov, Maxim Yu

May, James Michael

May, Karl Henrik

Mayer, Simon

Mayer, Thomas

Mayer-Gall, Thomas

Mayerhöfer, Thomas

Mayhew, Jerry

Maynadier, Anne

Maynar Mariño, Marcos

Mayor, David L.

Mayor, Jesus

Mayoral, Asunción M.

Mayorga-Martinez, Carmen C.

Mayr, Guenther

Mayuet, Pedro F.

Mayyahi, Ahmed Al 
Maździarz, Marcin

Mažeika, Dalius

Mazilescu, Crisanta-Alina

Mazoteras-Pardo, Victoria

Mazuelas, Santiago

Mazur, Grzegorz

Mazur, Jacek

Mazurek, Grzegorz

Mazurek, Przemysław

Mazurkiewicz, Dariusz

Mazur-Marzec, Hanna

Mazza, Antonio

Mazza, Daniela

Mazza, Fabio

Mazzaglia, Agata

Mazzanti, Valentina

Mazzaro, Gregory

Mazzeo, Domenico

Mazzeo, Giovanni

Mazzeo, Giuseppe

Mazzeo, Mina

Mazzeo, Pier Luigi

Mazzone, Marian

Mazzoni, Chiara

Mazzucato, Federico

Mazzucco, Gianluca

Mbey, Jean Aime

McCamley, John D.

McCann, Roy

McCarthy, Dennis D.

McCarthy, Lynda H.

McCash, Luthais

Mccay, Alistair

McClarren, Ryan

McCrae, John P.

McDermott, Christopher

McDonald, Robbie

McErlain-Naylor, Stuart

McEwan, Alistair

McFarlane, Nicole

McGinty, Joshua A.

McGrath, Andrew P.

McGrattan, Kevin B.

McGuire, Michael

McIlroy, Marie

McIntyre, Shelby

McKay, Jennifer

McKinley, Brett

McLagan, David

McLaughlin, John

McLaughlin, Patricia J.
McLellan, Iain

Mclennon, Everald

McMichael, Ian T

McMillan, Alison J.

McPheron, Benjamin D.

McRae, Jacqui M.

McVey, Michael

Me, Gianluigi

Meade, Douglas B

Méausoone, Pierre-Jean

Męczyńska-Wielgosz, Sylwia

Meda, Alice

Mêda, Pedro

Medarevic, Djordje

Meddaugh, W. Scott

Medeiros, Pedro

Medhat, Fady

Medic, Tomislav

Medina Carrasco, Santiago

Medina Quesada, Ángeles

Medina, David

Medina, Hector

Medina, Ruben

Medo, Juraj

Medrano, Carlos

Medrano, Marc

Medvecky, Lubomir

Medvedev, Sergey

Medvedev, Viktor

Medyńska-Juraszek, Agnieszka

Meek, Sanford

Meen, Teen-Hang

Meena, Nand K.

Meena, Naveen Kumar

Meenashisundaram, Ganesh Kumar

Meesenburg, Henning

Mefleh, Marina

Megahed, Mustafa

Megalooikonomou, Konstantinos G.

Meghil, Mohamed

Megret, Patrice

Megyesiová, Silvia

Mehadji, Abri

Mehdizadeh, Armifarhang

Mehmood, Faizan

Mehmood, Khawaja Khalid

Mehrabi, Armin

Mehta, Abhishek

Mehta, Michael

Mehta, Sunali

Mehulić, Ketij 
Mei, Hanfei

Mei, Jun

Mei, Lanju

Meiburger, Kristen M.

Meidute-Kavaliauskiene, Ieva

Meier, Robert J.

Meijide, Francisco

Meimandi, Kiana Jafari

Meir, Isaac A.

Meisen, Tobias

Meisina, Claudia

Meissner, Miroslaw

Mejzlik, Jan

Mekala, Rao Narasimha

Mekasha, Sophanit

Mekhemar, Mohamed

Mekhilef, Saad

Mekhonoshin, Aleksey

Mekki, O. Ben

Mekuchi, Miyuki

Mela, Petra

Mélard, Guy

Melcer, Jozef

Melchor, Juan

Mele, Elena

Mele, Giuseppe

Melero, Julio J.

Meletiou-Christou, Maria-Sonia

Melguizo-Guijarro, Manuel

Melían-Díaz, Dámari

Melini, Francesca

Melinte, Daniel Octavian

Melita, Marco

Melito, Lorenzo

Melkikh, Alexey V.

Melkonian, Jean-Michel

Mellouli, Nédra

Melnik, Rafał

Melnikov, Pavel

Melville, Owen A.

Memarzadeh, Milad

Memisoglu, Gorkem

Memmolo, Vittorio

MEMON, FAISAL AHMED

Memon, Saim

Mempin, Maria

Men, Yongjun

Men, Zhongxian

Ménard, Hervé

Menasalvas, Ernestina

Mendes, Jérôme
Mendes, Maria Paula

Mendes, Mário C.

Mendes, Nuno

Méndez, Roi

Mendez-Astudillo, Manuel

Méndez-Martínez, Carlos

Méndez-Suárez, Mariano

Mendikute, Alberto

Mendo, António

Mendonça, Tito

Menegatti, Emanuele

Meneghetti, Giovanni

Meneguzzo, Francesco

Menendez, Esther

Menendez, Hector

Menendez, Hector D.

Meng, Dong

Meng, Fanben

Meng, Lie

Meng, Maozhou

Meng, Meng

Meng, Qian

Meng, Wei

Meng, Weina

Meng, Weizhi

Meng, Xiang

Meng, Yifei

Meng, Yu

Meng, Yuan

Menga, Nicola

Mengarelli, Alessandro

Mengistu, Samson G.

Menichetti, Guido

Menichetti, Marco

Menini, Maria

Menoufi, Karim

Menshenin, Yaroslav

Mensi, Magda

Mensi, Mounir

Mentel, Grzegorz

Menter, Florian R.

Meo, Marianna

Meola, Carosena

Meoni, Gabriele

Mercader, Pedro

Mercader-Moyano, Pilar

Mercan, Ezgi

Mercer, John

Mercer, John A.

Merciol, François

Mercorelli, Paolo 


\author{
Merdasa, Aboma \\ Merdrignac, Pierre \\ Mergeay, Max \\ Merie, Violeta Valentina \\ Meringer, Markus \\ Merino-Martinez, Roberto \\ Merker, Jochen \\ Merkhouf, Arezki \\ Merla, Caterina \\ Merlin, Olivier \\ Merlo, Marco \\ Merlo, Vittorio \\ Mernik, Marjan \\ Merodio, Jose \\ Meroni, Gabrielle \\ Meroni, Giovanni \\ Merrick, Trina \\ Merten, Christian \\ Mertens, Christian \\ Mertzimekis, Theo \\ Merwood-Salisbury, Joanna \\ Merz, Rainer \\ Mesaros, Anca \\ Mesarovic, Sinisa \\ Mescola, Andrea \\ Meseguer, Victor Manuel \\ Meshram, Nirvedh \\ Mešić, Aleksandar \\ Mešić, Armin \\ Mesko, Maja \\ Mesodiakaki, Agapi \\ Messa, Gianandrea Vittorio \\ Messina, Barbara \\ Messina, Enza \\ Messineo, Daniela \\ Mester, Christian \\ Mestres, Narcís \\ Mestriner, Daniele \\ Metaxatos, Paul \\ Métioui, Abdeljalil \\ Metsis, Vangelis \\ Metz, Stefan W. \\ Metzler, Christopher \\ Meucci, Valentina \\ Meur, Olivier Le \\ Meyer, Alexandre \\ Meyer, Florent \\ Meynants, Guy \\ Mezei, József \\ Mezei, Mihaly \\ Mezzanotte, Valeria
}

Mezzetta, Andrea

Mezzina, Giovanni

Mezzini, Mauro

Mhiesan, Haider Ghazi

Mia, Mozammel

Miądlicki, Piotr

Miah, M Shamim

Miao, Chenyong

Miao, Hui

Miao, Ludi

Miao, Qiuhong

Miao, Yu

Miarka, Petr

Miąsik, Przemysław

Miatliuk, Kanstantsin

Micalizio, Salvatore

Miccadei, Enrico

Miccoli, Stefano

Micha, Dimitra

Michael, Adrian

Michael, Aron

Michael, Fredrick

Michail, Tsagris

Michail, Vaitis

Michalak, Aneta

Michalak, Piotr

Michalczyk, Małgorzata I.

Michalik, Kamil

Michalis, Panagiotis

Michalowska, Joanna

Michałowski, Bartosz

Michalska-Dudek, Izabela

Michalski, Jacek A.

Michalski, Jan

Michalski, Radoslaw

Michel, Magdalena

Michelini, Elena

Michihata, Masaki

Michopoulos, Apostolos

Michot, Benoit

Micillo, Raffaella

Micucci, Alfonso

Middei, Silvia

Midmore, David

Mie, Masayasu

Mieczkowski, Paweł

Miecznikowski, Krzysztof

Mielewczik, Michael

Mielewczyk-Gryn, Aleksandra

Mieloszyk, Magdalena

Mierzejewska, Żaneta Anna 
Miettinen, Jari

Migallón, Héctor

Migda, Wojciech

Migheli, Quirico

Migliori, Giovanni Battista

Migliorini, Sara

Mignon, Sandor

Migorski, Stanislaw

Miguel, Antonio

Miguel, Carlos V.

Mihaela, Andrei

Mihaescu, Cristian Marian

Mihai, Andrusca

Mihai, Bogdan Andrei

Mihai, Duguleana

Mihai, Ilie Valentin

Mihaicuta, Stefan

Mihaila, Ilarion

Mihailov, Nikolay

Mihalache, Sanda Florentina

Mihalcea, Bogdan Vasile

Mihali, Sorin

Mihalkovich, Aleksejus

Mihelič, Jurij

Mihelj, Jernej

Mihet-Popa, Lucian

Mihnea, Mihaela

Mikac, Lara M.

Mikada, Hitoshi

Mikala, Gabor

Mikalauskienè, Asta

Mikhailenko, Peter

Mikheev, Gennady M.

Mikielewicz, Dariusz

Mikkelsen, Ove

Miklaszewski, Andrzej

Miklos, Zoltan

Mikołajczak, Beata

Mikolajczyk, Tadeusz

Mikulchyk, Tatsiana

Mikulecký, Peter

Mikulski, Dawid

Mikuś, Paweł

Mikuš, Tomislav

Mikusova, Miroslava

Milan, Steve

Milani, Alfredo

Milani, Gabriele

Milani, Paolo

Milanic, Matija

Milano, Francesco
Milanova, Mariofanna

Milazzo, Maria Francesca

Milazzo, Mario

Milcarek, Ryan

Milciuviene, Saule

Mildren, Rich

Mile, Ivanda

Milecki, Andrzej

Miled, Zina Ben

Milella, Luigi

Mileti, Ilaria

Miletic, Ivana

Mileusnić, Marta

Milia, Egle

Milic, Vladimir

Milicevic, Ivana

Milicevic, Kruno

Milicevic, Mario

Milichovský, František

Milici, Salvatore

Milik, Adam

Milisav, Irina

Miljković, Miomir

Milkovič, Ondrej

Milla, Immonen

Miller, Alexander

Miller, Bartosz

Miller, Boris

Miller, David Michael

Miller, Gregory

Miller, Jimmie

Miller, Paul

Miller, Piotr

Miller, Reinhard

Miller, Tomasz

Miller, Wolfram

Mills, Amanda C.

Miloš, Müller

Milosan, Ioan

Milosavljević, Ivan

Milosevic, Slobodan

Milosz, Marek

Milsted, David

Miluski, Piotr

Min, Changgi

Min, Cheonhong

Min, Kyungha

Min, Rui

Minaee, Shervin

Minafo, Giovanni

Minak, Giangiacomo 
Minakshi, Manickam

Minami, Yoichi

Minasny, Budiman

Minav, Tatiana

Minažek, Krunoslav

Mindaugas, Gedvilas

Mîndrescu, Veronica

Minea, Alina Adriana

MINEA, Marius

Minelli, Alberto

Minematsu, Tsubasa

Mingant, Remy

Mingareev, Ilya

Mingesz, Robert

Mingorance-Estrada, Ángel Custodio

Mingotti, Alessandro

Minguez, Rikardo

Mínguez-Algarra, Jesús

Minguzzi, Alessandro

Minh, Le Van

Minic, Zeljka

Minichiello, Francesco

Minin, Igor V.

Minkiewicz, Piotr

Minn, Michael

Minna, Emanuela

Minnelli, Cristina

Minto, Riccardo

Mintz, Akiva

Minutolo, Vincenzo

Minzu, Viorel

Mir, Sajjad Husain

Miranda Melo, José Filipe

Miranda, Fabio

Miranda, Luis

Miranda, Pedro M. A.

Mirarchi, Claudio

Mirás Ávalos, Jose Manuel

Mirbeik-Sabzevari, Amir

Mirbozorgi, Abdollah

Mircea, Sasu Lucian

Mircea-Bogdan, Radac

Mirhashemi, Arash

Miricioiu, Marius

Miriello, Domenico

Mirlohi, Susan

Mirocha, Jeffrey D.

Miron, Anca

Mironeasa, Silvia

Mironescu, Monica

Mironica, Ionut
Mironova, Maria

Miroshnichenko, Andrey

Miroslaw, Wesolowski

Mirsayar, Mirmilad

Mirtaheri, Peyman

Mirzaali, Mohammad J.

Mirzakhani, Hooman

Mirzavand, Rashid

Misa, Rafał

Mishina, Yuji

Mishra, Aashwin

Mishra, Alaknanda

Mishra, Anand

Mishra, Avanish

Mishra, Dhanada Kanta

Mishra, Madhav

Mishra, Pawan Kumar

Mishra, Priyanka

Mishra, Rajesh

Mishra, Sambeet

Mishra, Santosh K.

Misiakos, Konstantinos

Misiunaite, Ieva

Miska, Marc

Miskiewicz, Janusz

Miskiewicz, Mikolaj

Miskowicz, Marek

Mistretta, Fausto

Mistry, Kamlesh

Misul, Daniela

Mišurec, Jiřŕ

Miszczak, Jaroslaw Adam

Miszczyk, Justyna

Mita, Koji

Mitani, Atsushi

Mitani, Tomohiko

Mitchell, Cassie S.

Mitev, Valentin

Mitew-Czajewska, Monika

Mitkowski, Piotr

Mitoulis, Stergios

Mitra, Mainak

Mitrica, Dumitru

Mitrin, Boris I.

Mitryukovskiy, Sergey

Mitsea, Anastasia

Mitsukura, Yasue

Mittal, Anuj

Mittal, Nitesh

Mittal, Saurabh

Mittlmeier, Thomas W. F. 
Mitura, Andrzej

Mityushev, Valdimir

Miura, Hiroyuki

MIURA, Taito

Miyamoto, Ryusuke

Miyata, Shogo

Miyazaki, Kouji

Miyazaki, Takahiko

Miyazaki, Tetsuro

Miyoshi, Norio

Mizerska-Kowalska, Magdalena

Mizerski, Krzysztof

Mizia-Stec, Katarzyna

Mizuochi, Hiroki

Mizutani, Tsukasa

Mizzi, Luke

Mkaouer, Mohamed Wiem

Mlkvik, Marek

Młynarczyk, Przemysław

Mlynek, Jaroslav

Mo, Chen

Mo, Fei

Mobed-Miremadi, Maryam

Mobili, Alessandra

Mobilia, Mirka

Mobilio, Marco

Mocanu, Dan-Adrian

Mocanu, Irina

Moccia, Massimo

Moccia, Sara

Mocek, Jan

Mochocki, Wojciech

Mocnik, Franz-Benjamin

Mocquet, Vincent

Moczulski, Wojciech

Modesti, Paolo

Modi, Nisarg

Modica, Francesco

Modoni, Gianfranco

Moeller, Peter D. R.

Moffatt, Peter

Mogale, Dnyaneshwar

Møgelmose, Andreas

Moghaddam, Hesam S.

Moghaddam, Saeed

Mognaschi, Maria Evelina

Moh, Sangman

Mohaisen, Manar

Mohajernia, Shiva

Mohamad, Abdulmajeed A.

Mohamed, Elamin
Mohamed, Ismail

Mohamed, Junaith S.

Mohamed-Seghir, Mostefa

Mohammad, Shaqfeh

Mohammadi, Ali

Mohammadi, Fazel

Mohammadi, Mohammad Ebrahim

Mohammed, Alyaa

Mohammed, Iman

Mohammed, Nishil

Mohan, Chilukuri K.

Mohan, Gowtham

Mohan, Midhun (Mikey)

Mohan, Velram Balaji

Mohanpurkar, Manish

Mohebbi, Amin

Mohenado, Angel

Mohr, Thomas

Mohsen, Marani Barzani

Mohseni, Parsian K.

Moir, Gavin

Moita, Ana

Mojiri, Amin

Mojtabai, Navid

Mojtahedi, Mohammad

Mok, Daniel Kam-Wah

Mok, Sung-Hoon

Mokhirev, Aleksandr

Molaei, Maedeh

Molardi, Carlo

Molari, Luisa

Molčan, Matúš

Mölders, Nicole

Moldogazieva, Nurbubu

Moldovan, Ioana

Moldovan, Mirela

Moldovanu, Simona

Moldoveanu, Florica

Mole, Nikolaj

Molero Chamizo, Andrés

Molimard, Jérôme

Molina, Andreu Carbó

Molina, Jose Francisco

Molina-González, María Dolores

Molinari, Andrea

Molinari, Nicola

Molina-Solana, Miguel

Molina-Torres, María-Pilar

Molinos, Eduardo Jose

Molla, Mamun

Molla-Aliod, Diego 
Moller, Ann Merete

Møller, Kasper T.

Möllerström, Erik

Mollica Nardo, Viviana

Mollineda Cárdenas, Ramón Alberto

Molnar, Arthur

Molnár, György

Molnar, Wolfgang

Molteni, Erika

Molter, Daniel

Molyneux, David

Momete, Daniela

Momose, Akishi

Momot, Alina

Monaci, Fabrizio

Monaco, Ernesto

Monahan, Kyle

Monaldo, Elisabetta

Monat, Jamie

Mondal, Bikash

Mondal, Dhiman

Mondal, Sudeepta

Mondal, Utpal Kumar

Mondello, Attilio

Mondeshki, Mihail

Mondol, Jayanta

Monea, Monica

Monetta, Tullio

Mongil-Manso, Jorge

Monica, Riccardo

Moñino, Antonio

Monkova, Katarina

Monokrousos, Nikolaos

Monroy, Claire

Monsalve, Alberto

Monsuez, Bruno

Monsurrò, Pietro

Montalto, Luigi

Montalvan-Burbano, Nestor

Montalvão, Diogo

Montana, Jessica

Montanari, Gian Carlo

Montanari, Marco

Montañés, Elena

Montaño Priede, José Luis

Montans, Francisco Javier

Montaruli, Graziano

Montecchio, Daniele

Monteiro Santos, Fernando

Monteiro, Eliseu

Monteiro, Sandra
Monteiro, Susana

Monteiro, Vítor

Montemezzi, Pietro

Montemurro, Marco

Montemurro, Nicola

Montenegro, Pedro Aires

Monterde, Juan

Monterroso-Checa, Antonio

Montes Morán, Miguel A.

Montesano, Francesco Fabiano

Montesarchio, Daniela

Montes-García, Verónica

Montes-Moran, Miguel A.

Montezuma, Paulo

Montezuma-Carvalho, Paulo

Montgomery, Jack

Monti, Alessio

Montiel, José María

Montinaro, Nicola

Montisci, Augusto

Montori, Federico

Montoya, Oscar Danilo

Montrésor, Silvio

Monts, David

Montuori, Rosario

Montusiewicz, Jerzy

Monzo, Carlos

Moo, Eng Kuan

Mookiah, Muthu Rama Krishnan

Moon, Chanwoo

Moon, Dae Woon

Moon, Hyeonjoon

Moon, Jiho

Moon, Jongsub

Moon, Jucheol

Moon, Kee S.

Moon, Kiwon

MOON, Seung Ki

Moon, Seung-Jae

Moore, Stephen Edward

Moore, Wayne

Moosavian, Amin

Moosmann, Julian

Moosmuller, Hans

Mora, Pierric

Morabito, Andrea Francesco

Morabito, Francesco Carlo

Moradshahi, Mehrad

Morais, Diogo

Moraleda Sepúlveda, Esther

Morales Maqueda, Miguel Ángel 
Morales Suárez-Varela, María M.

Morales, Antonio

Morales-Conde, Maria Jesús

Morales-Navarrete, Hernán

Morales-Vidal, Marta

Moramarco, Vincenzo

Morán Álvarez, Antonio

Morar, Anca

Moraru, Luminita

Morasso, Carlo

Moravčík, Roman

Moravej, Mohammadtaghi

Morawiec, Mateusz

Morcego, Bernardo

Mörchen, Manfred

Morcia, Caterina

Mordak, Arkadiusz

Morea, Donato

Moreira, Carla

Moreira, Cristiana

Moreirinha, Catarina

Morel, Pascal

Morenilla Jimeno-Morenilla, Antonio

Moreno Beltrán, Plácido

Moreno García, María N.

Moreno, Francisco

Moreno, Hilario Gómez

Moreno, Ignacio

Moreno, Miguel Ramirez

Moreno, Ramón

Moreno, Vidal

Moreno-Andrés, Javier

Moreno-Eguilaz, Juan Manuel

Moreno-Fernandez, Jorge

Moreno-García, Carlos

Moreno-Garcia, Isabel María

Moreno-Guerrero, Antonio-José

Moreno-Gutiérrez, Juan

Moreno-Muro, Francisco-Javier

Moreno-Perez, Victor

Morente Molinera, Juan Antonio

Morer-Camo, Paz

Moret-Tatay, Carmen

Moretti, Giacomo

Moretti, Isabelle

Moretti, Laura

Morettini, Micaela

Moretto, Ligia Maria

Moretto, Sandra

Moreu, Fernando

Morgado, António Miguel
Morganti, Pierfrancesco

Morgantini, Marcello

Morgiel, Jerzy

Morgunov, Igor

Mori, Akihisa

Mori, Kotaro

Mori, Masaaki

Mori, Mauro

Mori, Naoki

Mori, Ryota

Moribayashi, Kengo

Morikawa, Masahiro

Morikawa, Osamu

Morillas Gómez, Samuel

Morillas, Leandro

Morillo, Daniel Sánchez

Morimoto, Yasuhiko

Morineau, Thierry

Morini, Marco

Moriñigo, Daniel

Morinigo-Sotelo, Daniel

Morio, Jérome

Morita, Manabu

Morita, Naoki

Morita, Shigeki

Moritake, Takashi

Moritomo, Yutaka

Morkoc, Hadis

Moro, Alessandro

Moro, Christian

Moro, Enrico

Moro, Sérgio

Moron, Carlos

Moroń, Tomasz

Moroni, Davide

Moroni, Fabrizio

Moros, José García

Morosuk, Tatiana

Morotomi, Takahiko

Morouço, Pedro

Morovat, Reza

Morozan, Adina

Morozov, Evgeny

Morozov, Oleg

Morozov, Oleg G.

Morozova, Iuliia

Morozova, Ksenia

Morrey, Denise

Morris, Abigail

Morris, Tim

Morrison, Melanie 
Morrone, Biagio

Morrone, Pietropaolo

Morrow, David

Morse, Daniel

Morseletto, Piero

Morsy, Salem

Mortazavi, Farzam

Mortensen, Luke J.

Mosavi, Amir

Moscato, Vincenzo

Moschitta, Antonio

Mościcki, Tomasz

Moser, Georg

Moser, Kenneth

Moshiri, Mandaná

Moshizi, Sajad Abolpour

Moshou, Alexandra

Mosier, Rachel D.

Mosin, Sergey

Moskalik, Tadeusz

Moskvin, A.S.

Mosleh, Araliya

Mosolygó, Timea

Mosorov, Volodymyr

Mössner, Martin

Mostafa, Ahmad

Mostafaei, Habib

Mostafavi Yazdi, Seyed Jamaleddin

Mostarac, Petar

Mostoni, Silvia

Mostovshchikov, Andrei V.

Moszyński, Dariusz

Motamed, Cyrus

Motoasca, Emilia

Motola, Martin

Motoyama, Keiichi

Motra, Hem Bahadur

Motta, Gianmario

Mottaghipisheh, Javed

Mottola, Fabio

Motyka, Maciej

Mouga, Teresa

Moujaees, Samir

Moujir, Laila M.

Mouli, Ramasamy

Moulianitis, Vassilis

Moulianitis, Vassilis C.

Moulin, Bernard

Moulos, Vrettos

Mounaix, Patrick

Mouralova, Katerina
Mouratidis, Despoina

Mourdikoudis, Stefanos

Mourelle, M. Lourdes

Mourtzis, Dimitris

Mourzenko, Valeri

Mousas, Christos

Mousavi, S. Mostafa

Moussa, Dina

Moussas, Vassilios C.

Moutsopoulou, Amalia

MOUWAKEH, Moufid

Mouzourides, Petros

Mowe, Maxine

Moy, Peter

Moyà Alcover, Gabriel

Moya Rueda, Fernando

Moya, Antonio A.

Moya, Cristian

Moya, JoséS Serafín

Moya, Manuel

Moyano Campos, Juan José

Moyne, Le

Moyseowicz, Adam

Moysis, Lazaros

Mozejko-Ciesielska, Justyna

Mozetič, Igor

Mozgovoy, Maxim

Mozzon, Mssimo

Mrabet, Hichem

Mračková, Eva

Mrakovčić, Tomislav

Mramor, Katarina

Mroczkowska-Szerszeń, Maja

Mroczyński, Robert

Mrówczyńska, Lucyna

Mrówczyńska, Maria

Mrowiec, Bozena

Mróz, Sebastian

Mróz, Zenon

Mrozek, Dariusz

Mrozik, Karol Dawid

Mrugalska, Beata

Mrvelj, Štefica

Mrzljak, Vedran

Mtz Khramer, Daniel

$\mathrm{Mu}$, DeKui

$\mathrm{Mu}$, Ruipu

Mu, Xuan

Mubbasir, Kapadia

Mucchetti, Germano

Mucchi, Lorenzo 
Mucha, Jacek

Mucherino, Antonio

Mück, Michael

Múčka, Peter

Mücke, Manfred

Muga, Gonzalo

Muguda, Sravan

Mugume, Rodgers

Muhammad, Mannan Saeed

Muhammad-Sukki, Firdaus

Muhlethaler, Paul

Mujica, Gabriel

Mukai, Hiroaki

Mukerji, Tapan

Mukherjee, Dibyendu

Mukherjee, Falguni

Mukherjee, Maitreyee

Mukherjee, Pritam

Mukhin, Nikolay

Mukhopadhyay, Partha

Mukhopadhyay, Subhas

Mukhopadhyay, Tanmoy

Mukkamala, Raghava Rao

Mulherkar, Shalaka

Mullaliu, Angelo

Mullally, Susan

Müller, Andrei

Müller, Bert

Müller, Claas

Müller, Danny

Müller, Ingmar

Müller, Joachim

Müller, Johannes

Müller, Julian Marius

Müller, Lutz

Müller, Phillip

Müller, Timo

Müller, Wolfgang $\mathrm{H}$.

Müller-Alcazar, Anett

Mullins, Darragh

Multari, Salvatore

Mummolo, Giovanni

Mumolo, Enzo M.

Mumtaz, Shahid

Mun, Sang Don

Munaron, Luca

Munch, Jean Charles

Münch-Alligné, Cécile

Muneer, Tariq

Munehiro, Kazunori

Munialo, Claire D.
Munir, Khushboo

Muniraj, Nethaji

Muniraj, Thiruvengadam

Muñiz-Calvente, Miguel

Munková, Daša

Muñoz Arnanz, Juan

Muñoz Hernández, Germán Ardúl

Muñoz, Antonio

Muñoz, José J.

Muñoz-Bañón, Miguel Á.

Muñoz-Gea, Juan Pedro

Muñoz-González, Carmen Ma

Munoz-Guijosa, Juan Manuel

Munoz-Organero, Mario

Munoz-Pinto, Dany

Muñoz-Saavedra, Luis

Munshi, Amit

Muntean, Gabriel-Miro

Muntean, Radu

Muntean, Sebastian

Munteanu, Calin

Munteanu, Florentina-Daniela

Munz, Michael

Mur, Angel

Muradova, Aliki

Murakami, Kosuke

Muralha, José Delgado

Murari, Krishna

Murata, Jun

Murata, Kazutoshi

Murawski, Lech

Murayama, Riichi

Muresan, Cristina

Muresan, Iulia

Muresan, Mircea Paul

Muresan, Vlad

Murkin, Andrew

Murotani, Kenta

Murphy, Adrian

Murphy, Anthony

Murphy, Cormac

Murray, Cameron

Murray, Harry

Murray, Steven Ross

Murrieta-Mendoza, Alejandro

Murshid, Nimer

Murtagh, Fionn

Murthy, Tammana S. R. C.

Murtomäki, Lasse

Murugan, Nirosha J.

Murugappan, Krishnan 


\begin{tabular}{|c|c|}
\hline Murugathasan, Latheepan & Myridakis, Antonis \\
\hline Murzin, Anton & Mystkowska, Joanna \\
\hline Musa, Sarhan & Mystkowski, Arkadiusz \\
\hline Musca, Rossano & Myszograj, Sylwia \\
\hline Muscio, Alberto & Na, Wonbae \\
\hline Muscolo, Giovanni Gerardo & Nabajeet, Barman \\
\hline Musgrave, Christopher Stephen Andrew & Nabavi, Ali \\
\hline Musiał, Michał & Nabavi, Seyedfakhreddin \\
\hline Musicant, Oren & Nabavizadeh, Reza \\
\hline Muskeri, Saideep & Nabiałek, Marcin \\
\hline Muslimov, Eduard & NABIL, BACHAGHA \\
\hline Musmarra, Dino & Nabil, Mahmoud \\
\hline Musolino, Antonino & Nabizadeh, Azam \\
\hline Mussi, Elisa & Naccache, Rafik \\
\hline Musso, Maurizio & Naceri, Abdeldjallil \\
\hline Musso, Stefano & Nacheva, Radka \\
\hline Mustafa, Mustafa & Nadai, Akitsugu \\
\hline Mustapha, Samir & Naddeo, Alessandro \\
\hline Musteata, Mihai & Nadeau, Jay L. \\
\hline Mustoe, George E. & Nadeem, Muhammad \\
\hline Musumeci, Francesco & Naderi, Ehsan \\
\hline Musumeci, Giuseppe & Naderi, Esmaeil \\
\hline Musumeci, Rosaria Ester & Naderian, Ali \\
\hline Musumeci, Salvatore & Nadimi, Mohammad \\
\hline Muszynski, Larry & Nadolny, Zbigniew \\
\hline Muszyński, Siemowit & Naeem, Umair \\
\hline Muszyński, Tomasz & Nafis, Ahmed \\
\hline Muthu Karuppan, Mohan Kumar & Naftaly, Mira \\
\hline Muthugala, Viraj & Nag, Soumyadeep \\
\hline Muthuraj, Rajendran & Nagahage, Isura \\
\hline Muthuraman, Parthasarathy & Nagai, Hiroki \\
\hline Mutilba, Unai & Nagai, Norihiro \\
\hline Muto, Masaya & Nagam Hanumantharao, Samerender \\
\hline Mutreja, Isha & Nagano, Hanatsu \\
\hline Mutschler, Christopher & Nagano, Tatsuya \\
\hline Mutshinda, Crispin & Nagaoka, Isao \\
\hline Muttoni, Aurelio & Nagarajan, Ragupathi \\
\hline Muydinov, Ruslan & Nagata, Koji \\
\hline Muyor, José M. & Nagatani, Keiji \\
\hline Muzio, Lorenzo Lo & Nagatani, Naoki \\
\hline Muziol, Tadeusz Mikolaj & Nagatoishi, Satoru \\
\hline Muzyk, Marek & Naghdi, Samira \\
\hline Mwesigye, Aggrey & Naghilou, Aida \\
\hline Myalska, Hanna & Naghshvarianjahromi, Mahdi \\
\hline Myasoedova, Tatiana N. & Nagit, Gheorghe \\
\hline Myeong, Soojeong & Nagle, Anna \\
\hline Myers, Mike & Naglic, Peter \\
\hline Myers, Oliver & Nagy, Bálint \\
\hline Myers, Stephen David & Nagy, Dávid U. \\
\hline Myler, Harley R. & Nagy, Elod \\
\hline Myler, Peter & Nagy, Előd Ernő \\
\hline
\end{tabular}




\begin{tabular}{|c|c|}
\hline Nagy, Mariana & Nallasamy, Palanisamy \\
\hline Nagy, Miklós & Nalli, Giacomo \\
\hline Nagy, Szilvia & Nalmpantis, Dimitrios \\
\hline Nah, Wansoo & Nam, Juhan \\
\hline Nahata, Sudhanshu & Nam, Ki Hyun \\
\hline Nahid, Abdullah-Al & Nam, Kwang Woo \\
\hline Nahidi, Asal & Nam, Yunyoung \\
\hline Nahvi, Ali & Nama Manjunatha, Krishna \\
\hline Nai Ruscone, Marta & Namazi, Hamidreza \\
\hline Naik, Akshata & Nambiar, Dhanya K. \\
\hline Naik, Punith & Nambu, Yasusada \\
\hline Naik, Vanaja Krishna & Namgung, Ho \\
\hline Nair, Arun Sukumaran & Namysłowska-Wilczyńska, Barbara \\
\hline Nair, Rathin Radhakrishnan & Nanaki, Stavroula \\
\hline Naito, Katsuyuki & Nanako, Kawaguchi \\
\hline Naito, Toshio & Nandi, Dip \\
\hline Najafi, Foad & Nanjappa, Deepak \\
\hline Najahi Missaoui, Wided & Nanjundan, Meera \\
\hline Najarian, Siamak & Nanni, Gabriele \\
\hline Najdahmadi, Avid & Nansai, Shunsuke \\
\hline Nájera López, Alberto & Naoki, Miyazato \\
\hline NAJM, HUSAM S. & Napari, Mari \\
\hline Nakagawa, Seiichi & Napieralska-Juszczak, Ewa \\
\hline Nakahara, Hiroki & Napiórkowski, Jerzy \\
\hline Nakahata, Kazuyuki & Naplocha, Krzysztof \\
\hline Nakai, Kunihiko & Napoli, Christian \\
\hline Nakaki, Toshio & Napoli, Marco \\
\hline Nakamae, Koji & Napolitano, Rebecca \\
\hline Nakamoto, Tetsuo & Näppi, Janne J. \\
\hline Nakamura, Hisahide & Náprstková, Nataša \\
\hline Nakamura, Takashi & Narancic, Biljana \\
\hline Nakamura, Tomoki & Naranjo, Jose Eugenio \\
\hline Nakanishi, Yoshitaka & Naranjo-Zeledón, Luis \\
\hline Nakano, Tadashi & Narayanan, Barath \\
\hline Nakarmi, Ukash & Nardello, Francesca \\
\hline Nakarmi, Upama & Nardi, Iole \\
\hline Nakashima, Motomu & Nardini, Giovanni \\
\hline Nakata, Yosuke & Nardini, Sergio \\
\hline Nakatani, Yoshihiko & Nardo, Dario Di \\
\hline Nakatsuhara, Katsumi & Narducci, Dario \\
\hline Nakayama, Akira & Narducci, Fabio \\
\hline Nakayama, Yuushou & Naris, Steryios \\
\hline Nakayasu, Ernesto & Narkar, Ameya \\
\hline Nakhli Mahal, Houman & Narkiewicz, Janusz \\
\hline Nakonieczny, Damian & Narloch, Piotr \\
\hline Nalakath, Haris & Naro, Antonino \\
\hline Nalbandian, Michael J. & Narojczyk, Jakub W. \\
\hline Nalepa, Grzegorz & Narracott, Andrew J. \\
\hline Nalepa, Jakub & Narsipur, Shreyas \\
\hline Nalewajko, Joanna Sekulska & Narzisi, Antonio \\
\hline Nalivaiko, Anton Yu. & Nasakina, Elena O. \\
\hline
\end{tabular}

Nagy, Mariana

Nagy, Miklós

Nagy, Szilvia

Nah, Wansoo

Nahata, Sudhanshu

Nahid, Abdullah-Al

Nahidi, Asal

Nahvi, Ali

Nai Ruscone, Marta

Naik, Akshata

Naik, Punith

Naik, Vanaja Krishna

Nair, Arun Sukumaran

Naito, Katsuyuki

Naito, Toshio

Najafi, Foad

Najahi Missaoui, Wided

Najarian, Siamak

Najdahmadi, Avid

Nájera López, Alberto

NAJM, HUSAM S.

Nakagawa, Seiichi

Nakahara, Hiroki

Nakahata, Kazuyuki

Nakai, Kunihiko

Nakaki, Toshio

Nakamae, Koj

Nakamoto, Tetsuo

Nakamura, Hisahide

Nakanishi, Yoshitaka

Nakano, Tadashi

Nakashima, Motomu

Nakata, Yosuke

Nakatani, Yoshihiko

Nakatsuhara, Katsumi

Nakayama, Akira

Nakayama, Yuushou

Nakayasu, Ernesto

Nakhli Mahal, Houman

Nakonieczny, Damian

Nalakath, Haris

Nalbandian, Michael J.

Nalepa, Grzegorz

Nalivaiko, Anton Yu.
Nallasamy, Palanisamy

Nalli, Giacomo

Nalmpantis, Dimitrios

Nam, Juhan

Nam, Ki Hyun

Nam, Kwang Woo

Nam, Yunyoung

Nama Manjunatha, Krishna

Namazi, Hamidreza

Nambiar, Dhanya K.

Namgung, Ho

Namysłowska-Wilczyńska, Barbara

Nanaki, Stavroula

Nandi, Dip

Nanjappa, Deepak

Nanjundan, Meera

Nanni, Gabriele

Nansai, Shunsuke

Naoki, Miyazato

Napari, Mari

Napieralska-Juszczak, Ewa

Napiórkowski, Jerzy

Naplocha, Krzysztof

Napoli, Christian

Napoli, Marco

Napolitano, Rebecca

Näppi, Janne J

Naranjo, Jose Eugenio

Naranjo-Zeledón, Luis

Nardi, Iole

Nardini, Giovanni

Nardini, Sergio

Nardo, Dario Di

Narducci, Dario

Narducci, Fabio

Naris, Steryios

Narkar, Ameya

Narkiewicz, Janusz

Narloch, Piotr

Naro, Antonino

Narojczyk, Jakub W

Narracott, Andrew J.

Narzisi, Antonio

Nasakina, Elena O. 


\begin{tabular}{|c|c|}
\hline Nascimbene, Roberto & Nazir, Aamer \\
\hline Nascimento, Maria M. & Nazir, Salman \\
\hline Naseer, Prof. Noman & Nazir, Tariq \\
\hline Nasiłowska, Barbara & Naznin, Most Tahera \\
\hline Nasimuddin, N. & Ndegwa, Eunice N. \\
\hline Nasir, Mashood & Ndieyira, Joseph \\
\hline Nasiri, Sara & Ndzi, David \\
\hline Nasry, Walaa & Neagoe, Mircea \\
\hline Nassauer, Anne & Neagu, Adrian \\
\hline Nassehi, Aydin & Neagu, Bogdan Constantin \\
\hline Nassour, John & Neagu, Daniel \\
\hline Nastac, Silviu & Neagu, Dragos \\
\hline Nastasi, Benedetto & Neagu, Gabriel \\
\hline Nasti, Rita & Neamtu, Calin \\
\hline Nastri, Elide & Neasham, Jeffrey \\
\hline Nastro, Alessandro & Nebro, Antonio \\
\hline Nastuta, Andrei Vasile & Nečas, David \\
\hline Natafgi, Nabil & Necas, Tomas \\
\hline Nataliani, Yessica & Nečasová, Barbora \\
\hline Nath, Arijit & Necchi, Monica \\
\hline Nathan, Fox & Nechita, Elena \\
\hline Natili, Francesco & NECHITA, FLORIN \\
\hline Natkaniec-Nowak, Lucyna & Nęcka, Edward \\
\hline Natsuaki, Ryo & Nęcka, Krzysztof \\
\hline Nauroze, Syed Abdullah & Necsulescu, Dan-Sorin \\
\hline Nautiyal, Amit & Necula, Sabina \\
\hline Nava-Baro, Enrique & Nedelchev, Lian \\
\hline Navarrete, Miguel & Nedelcu, Anisor \\
\hline Navarro, Elena & Nedelcu, Dorian \\
\hline Navarro, Francisco J. & Nedelcu, Mihai \\
\hline Navarro, Roberto & Nedeljković, David \\
\hline Navarro-Camba, Enrique A. & Nedeljković, Marija \\
\hline Navarro-Esbrí, Joaquín & Nee, Andrew \\
\hline Navarro-Lopez, Vicente & Neelakantan, Prasanna \\
\hline Navarro-Tapia, Diego & Neerukonda, Sabarinath \\
\hline Navas, Javier & Nees, Matthias \\
\hline Navas-Delgado, Ismael & Nefedov, Aleksey A. \\
\hline Navas-Montilla, Adrian & Neghină, Cătălina \\
\hline Navia, Marlon & Negishi, Nobuaki \\
\hline Naviglio, Daniele & Negoro, Hiromitsu \\
\hline Nawani, Pranav & Negrea, Adina \\
\hline Nawar, Said & Negrean, Iuliu \\
\hline Naya Villaverde, Miguel Ángel & Negru, Mihai \\
\hline Naydenova, Izabella & Negut, Irina \\
\hline Nazarchuk, Evgeny & Neighbour, Gareth \\
\hline Nazari-Sharabian, Mohammad & Neilson, Roy \\
\hline Nazarko, Joanicjusz & Neipp, Cristian \\
\hline Nazarko, Piotr & Neiva, Henrique P. \\
\hline Nazarov, Dmitry Mikhailovich & Nejad, Ali Farokhi \\
\hline Nazarov, Yuli V. & Nejbert, Krzysztof \\
\hline Nazdrowicz, Jacek & Neji, Mohamed \\
\hline
\end{tabular}


Nejman, Alicja

Nelaturu, Phalgun

Nelatury, Sudarshan

Nelson, Alexander $\mathrm{H}$.

Nelson, Carl

Nelson, Chas

Nelson, David

Nelson, Jared W.

Nemchinova, Nina

Němec, Miroslav

Nemec, Mitja

Nemes, Ciprian

Nemoto, Koji

Nemoto, Takuma

Nemova, Darya

Nemś, Magdalena

Nemzer, Louis R.

Neog, Debanga Raj

Nepal, Ramesh

Nepeina, Kseniia

Neri, Matteo

Nermend, Kesra

Nesje, Atle

Nesmachnow, Sergio

Nesticò, Antonio

Nestler, Ulf

Nestorović, Tamara

Nestrasil, Igor

Netek, Rostislav

Neto, Maria

Nettelblad, Carl

Netzelmann, Udo

Neubauer, Reinhard

Neuenschwander, Juerg

Neufeld, Janis S.

Neugebauer, Maciej

Neuman, Tomas

Neumann, Claus

Neumann, Cornelius

Neumann, Niels

Neunaber, Claudia

Neunaber, Ingrid

Neuner, Matthias

Neunert, Grazyna

Neusypin, Konstantin A.

Nevalainen, Paavo

Neves, João C.

Neves, José

Nevidomskaya, Dina G.

Nevshupa, Roman A

Newbold, Joseph
Newcomb, Robert

Newe, Thomas

Newman, Steven E.

Newmire, Daniel

Newson, Alasdair

Newton, Michael

Nezamabadi, Saeid

Nežerka, Václav

NF, Kamaruzzaman

$\mathrm{Ng}$, Alex

$\mathrm{Ng}$, Brian

$\mathrm{Ng}$, Ding-Quan

$\mathrm{Ng}$, Tang-Tat

$\mathrm{Ng}$, Wei Long

Ngamkhanong, Chayut

Ngamsombat, Chanon

Nge, Thi Thi

Ngo, Anh H.

Ngo, Chunyong

Ngo, Ha Duong

Ngo, Tri Dung

Ngo, Trung Ngoc

Ngoc-Phi, Nguyen

Ngomo, Suzy

Nguyen Tien, Dat

NGUYEN, Anh-Tu

Nguyen, Binh

Nguyen, Chuong V.

Nguyen, Dinh Tung

Nguyen, Dong-Nhat

Nguyen, Duc-Hiep

Nguyen, Duc-Phuc

Nguyen, Giang

Nguyen, Hai

Nguyen, Hoang

Nguyen, Hung

Nguyen, Kien

Nguyen, Linh

Nguyen, Lu Trong Khiem

Nguyen, Luong Vuong

Nguyen, Nha

Nguyen, Nhu-Van

Nguyen, Quang Ngoc

Nguyen, Son Thanh

Nguyen, Sy-Ngoc

Nguyen, Thanh Duc

Nguyen, Tien-Dung

Nguyen, Tin

Nguyen, Trieu

Nguyen, Trong-Nguyen

Nguyen, Trung 
Nguyen, Tu

Nguyen, Tuan T.

Nguyen, Van Linh

Nguyen, Vu-Hieu

Nguyen-Van, Triet

$\mathrm{Ni}$, Junjun

$\mathrm{Ni}$, Yiqing

$\mathrm{Ni}$, Zhijiang

Niavis, Spyros

Niazi, Imran Khan

Niazmand, Amirreza

Nica, Petru

Nicassio, Francesco

Niccolai, Alberto

Niccolini, Gianni

Nicholas, Darrel

Nicholson, John W.

Nick, Hamid

Nicoara, Mircea

Nicoară, Mircea N.

Nicodemo, Gianfranco

Nicolaescu, Ioan

Nicolai, Marisa

Nicolas, Andrea

Nicolas-Silvente, Ana Isabel

Nicolau, Cristina

Nicolazzo, Serena

Nicolella, Maurizio

Nicolet, Pierrick

Nicoletti, Rosario

Nicoletti, Sergio

Nicolini, Andrea

Nicoll, Amanda J.

Nicolosi, Vittorio

$\mathrm{Nicu}$, Ionut Cristi

Nieć, Jakub

Nieckarz, Zenon

Niedbalski, Zbigniew

Nieddu, Giovanni

Niedoba, Tomasz

Niedzielski, Przemysław

Niedźwiecki, Jacek

Niegodajew, Paweł

Niehoff, Philip

Nielsen, Dwayne

Nielsen, Karl

Nielsen, Vilhjálmur

Niemann, Jorg

Niemczewska-Wojcik, Magdalena

Niemeier, Andreas

Nieminen, Kaarlo
Nieminen, Pentti

Nieminen, Tiina $\mathrm{M}$.

Niemitz, Jeffrey W.

Nieschulze, Jens

Niespodziana, Katarzyna

Nieße, Astrid

Niessen, Frank

Nieto Escámez, Francisco

Nieto, Andy

Nieus, Thierry R.

Nieuwazny, Jagna

Nieuwendaal, Ryan C.

Nieuwenhuizen, Theo

Nieves, Elena Hernández

Nieves, Javier

Niewiadomski, Wiktor

Niewiara, Lukasz

Niezgoda-Zelazko, Beata

Niffenegger, Markus

Nifli, Artemissia-Phoebe

Nigim, Khaled

Nigro, Valentina

Niguchi, Noboru

Niguès, Antoine

Nihei, Naoto

Nik-Bakht, Mazdak

Nikiforova, Anastasija

Niki-Maleki, Khashayar

Nikishin, Sergey

Nikitas, Alexandros

Nikitas, Nikolaos

Nikitin, Yury Rafailovich

Nikkhah, Amin

Nikkhah, Hamid Reza

Niknafs, Shahram

Nikodem, Maciej

Nikodem, Michał

Nikolaev, Dmitry

Nikolaidis, Alexandros K.

Nikolaidis, Athanasios

Nikolaidis, Th.N.

Nikolaivits, Efstratios

Nikolakopoulos, Konstantinos

Nikolakopoulos, Pantelis G.

Nikolaos, Karathanasopoulos

Nikolaou, Triantafyllia G.

Nikoleli, Dimitrios P.

Nikolelis, Dimitrios P.

Nikolic, Danijela

Nikolic, Jelena

Nikolopoulos, Dimitrios 
Nikolov, Ivan Adriyanov

Nikolova, Maria

Nikolovski, Srete

Nikończuk, Piotr

Nikouli, Eleni

Nikulin, Alex

Nikulin, Yury

Nikumb, Suwas

Nilforoushan, Niloufar

Nilfouroushan, Faramarz

Nilsson, Tobias

Nimbalkar, Sanjay

Nimma, Kutaiba Sabah

Nimmagadda, Shastri

Nina, Aleksandra

Nina, Brutch

Ning, Jinqiang

Niola, Vincenzo

Niri, Mona Faraji

Nirwane, Abhijit

Nischwitz, Sebastian P

Nishi, Kosuke

Nishi, Yoshiki

Nishikawa, Yuichi

Nishimoto, Yoshio

Nishimura, Tsutomu

Nishino, Hiroki

Nishio, Mayuko

Nishio, Mizuho

Nishitsuji, Takashi

Nishizawa, Tatsuo

Nissen, Lorenzo

Nistor, Cristina

Nistor, Ileana Denisa

Nistor, Sorin

Nitaj, Abderrahmane

Nitsos, Christos K.

Nitta, Naotaka

Nitulescu, Mihai

Nitzke, Jean

Nitzsche, Bianca

Nitzsche, Nico

Niu, Tianhua

Niu, Yuling

Nivolianitou, Zoe

Niyaz, Quamar

Nizamov, Shavkat

Njegovec, Matej

Njoroge, Anastasia

Nnaji, Chukwuma

Nobile, Adriano
Nobile, Lucio

Nobile, Riccardo

Nobili, Andrea

Noble, Mark S.

Noblin, Alice

Nobre, Ana

Nobukawa, Teruyoshi

Nocente, Massimo

Nocera, Francesco

Nocera, Luciano

Nodera, Hiroyuki

Nofal, Eslam

Noga, Marcin

Noga, Stanisław

Nogales-Bueno, Julio

Nogami, Kenji

Noge, Koji

Nogueira, Paulo

Noh, Yoojeong

Nojiri, Shuko

Nojoumian, Mehrdad

Nokhbeh, Reza

Nolan, Michael

Nonaka, Hirofumi

Nonappa, Nonappa

Nonato, Maddalena

Nonis, Francesca

Noor E Khuda, Sarkar

Noor, Nuruzzaman

Nooraiepour, Mohammad

Noori, Farzan Majeed

Noori, Mohammad

Norcic, Gregor

Noro, Marco

Noronha, Fernando

Norouzi, Amir

Norström, Fredrik

North, Max M.

Northrop, Paul

Nosek, Radovan

Noshad, Morteza

Nosko, Oleksii

Nosrati, Mehdi

Nota, Alessandro

Notaro, Immacolata

Notti, Emilio

Nouh, Mostafa

Nouizi, Farouk

Nouri, Amin

Nouri, Hassan

Noussan, Michel 
Novačko, Luka

Novais, Rui Miguel Teixeira

Novak, Andrej

Novák, Josef

Novak, Jurica

Novák, Viliam

Novellino, Alessandro

Novello, Vittorino

Novickij, Jurij

Novickij, Vitalij

Novo, André

Novo, Luís

Novoa, José

Novotná, Beáta

Novotný, Michal

Novroski, Nicole

Nowacka, Malgorzata

Nowaczyk, Jacek

Nowak, Agnieszka

Nowak, Krzysztof

Nowak, Michal S.

Nowak, Robert

Nowak, Sascha

Nowak-Michta, Aneta

Nowakowska, Justyna

Nowakowska-Lipiec, Katarzyna

Nowakowski, Piotr

Nowak-Woźny, Dorota

Nowamooz, Hossein

Nowicka, Elzbieta

Nowicka-Krawczyk, Paulina

Nowicki, Marek

Nowicki, Michał

Nowogonska, Beata

Nowoświat, Artur

Nowotny, Łukasz

Nowrot, Andrzej

Nozomi, Takeuchi

Nozzoli, Francesco

Ntaikou, Ioanna

Ntantogian, Christoforos

Ntemiri, Alexandra

Ntislidou, Chrysoula

Ntouskos, Valsamis

Nubani, Linda

Nuchkrua, Thanana

Nüchter, Andreas

Nucita, Andrea

Numata, Yasushi

Nunes, João Pedro

Nunes, Leonel
Nunes, Maria Fátima

Nuñez, Alberto

Núñez, Alfredo

Nuñez, Neftali

Nunez, Oscar

Núñez, Remigio Paradelo

Nuñez-Andrés, Amparo

Núñez-Valdez, Edward R.

Nunzianna, Doti

Nurmi, Jari

Nürnberg, Dennis

Nurse, Jason R. C.

Nurzynska, Karolina

Nusenu, Shaddrack Yaw

Nuster, Robert

Nuță, Diana Camelia

Nuti, Camillo

Nutile, Samuel

Nutskova, Mariia V.

Nuzzo, Stefano

Nwaiwu, Ogueri

Nyang'au, Wilson Ombati

Nycz, Andrzej

Nycz, Jacek

Nyka, Krzysztof

Nyongesa, Kevin W.

Nyssen, Florence

Nyström, Alexander

O'Banion, Matt

O'Brien, Megan K.

O'Brien, Paul

O'Byrne, Michael

O'callaghan, Yvonne

O'Connell, Kathleen

O'Duill, Sean

O'Faolain, Liam

O'Grady, Mathew

O'Keeffe, Gary

O'Loughlin, Declan

O'Mahony, Donal

O'Neal, Christine Knoblauch

O'Reilly, Gerard J.

O'Shaughnessy, Douglas

O'Sullivan, Thomas

O'Toole, Martin G.

Oancea, Bogdan

Oancea, Gheorghe

Oats, Renee

Obaidat, Muath A.

Obara, Paulina

Obata, Kotaro 
Obataya, Eiichi

Obeid, Muhannad

Obeng-Gyasi, Emmanuel

Oberman, Tin

Obolkin, Vladimir

Obregon, Josue

Obrero Gaitán, Esteban

Obst, Maciej

Obst, Marcus

Obuchowicz, Andrzej

Obuchowski, Romuald

Obukhov, Valery

Obushevs, Artjoms

Ocampo, Carlos A Rios

Ochiai, Masayuki

Ochieng, Washington Yotto

Ochowiak, Marek

Oda, Shoji

Odagiu, Antonia

Odeh, Saad

Odeh, Suhail M.

Odening, Katja

Odeyemi, Olumide A.

Odinokov, Sergey

Odrobiňák, Jaroslav

Odry, Akos

Odziemczyk, Waldemar

Oehlschlaeger, Matt

Oehring, Daniela

Oettingen, Mikolaj

Ofoegbu, Stanley Udochukwu

Ogashawara, Igor

Ogawa, Akiko

Ogawa, Fumio

Ogawa, Keiji

Ogawa, Keiko

Ogbourne, Steven

Oggeri, Claudio

Ogihara, Mitsunori

Ogirigbo, Okiemute Roland

Ogiso, Satoki

Ogorek, Rafal

Ogras, Umit

Ogrodnik, Karolina

Oguchi, Takashi

Ogut, Mehmet

Oh, Dahyun

Oh, Eunsung

Oh, Heekuck

Oh, Hyunchul

Oh, HyungSeon
Oh, In-Jae

Oh, Jaehyeon

Oh, Jaewon

Oh, Jae-Wook

Oh, Jeong-Ho

Oh, Jonghyun

Oh, Jong-Seok

Oh, JungHwan

Oh, Junho

Oh, Ki Yong

Oh, Kwangseok

Oh, Kyeong Keun

Oh, Sang-Keun

Oh, Seaseung

Oh, Seung Jae

Oh, Taekeun

Ohara, Keishi

Ohayon, Roger

Ohba, Seigo

Öhberg, Fredrik

Ohmagari, Shinya

Ohno, Nobutada

Ohno, Seigo

Ohsawa, Yukio

Ohta, Mitsuhiro

Ohta, Shinji

Ohtaka, Atsushi

Ohto, Tatsuhiko

Ohtsu, Masayasu

Ohyama, Tetsuo

Ojeda-May, Pedro

Ojha, Chandrakanta

Ojo, Adegbola

Ojovan, Michael I

Ojsteršek, Robert

Ojwang, Dickson

Ok, Gyeongsik

Okada, Isamu

Okafor, Florence

Okamoto, Hiroyuki

Okamoto, Satoru

Okamoto, Shunsuke

Okamoto, Takuma

Okarma, Krzysztof

Oke, Adekunle

Okiji, Takashi

Okorie, Okechukwu

Okorokov, Volodymyr

Okpin, $\mathrm{Na}$

Okrajni, Jerzy

Oks, Eugene 
Okubadejo, Olumide

Okumura, Dai

Okumura, Nobuaki

Okumura, Susumu

Okumura, Tadayoshi

Okunade, Adewole L.

Okunkova, Anna A.

Okyay, Ünal

Olafsdottir, Elin Asta

Olafsen, Sigmund

Olagunju, Kehinde Oluseyi

Olah, Branislav

Olanipekun, Ayokunle

Olanipekun, Ayokunle Olubunmi

Olarte-Mantilla, Sandra

Olatunbosun, Oluremi Ayotunde

Olazagoitia, José Luis

Olchowka, Jacob

Olczykowski, Zbigniew

Oldfield, Philip

Olejar, Kenneth J.

Olejarczyk, Michał

Olejniczak, Alicja

Olejniczak, Teresa

Olejnik, Bartosz

Olejnik, Paweł

Oleksandrovych, Illiashenko Oleg

Oleniacz, Robert

Olfat, Hamed

Olfs, Hans-Werner

Olga, Doroshenko

Oliva, Gabriele

Oliva, Pietro

Olivares, Lucio

Oliva-Teles, Maria Teresa

Oliveira Panão, Miguel R.

Oliveira Pinto, José Gabriel

Oliveira, Anabela

Oliveira, Irene

Oliveira, Joana

Oliveira, Jorge

Oliveira, Luciana

Oliveira, Marta

Oliveira, Miguel

Oliveira, Monica

Oliveira, Rúben

Oliveira, Serafim M.

Oliveira, Teresa A.

Oliver, Lisa

Oliver, Sergio

Olivera, Vicente Matellán
Oliveto, Giuseppe

Olivetti, Elena

Oliviero Rossi, Cesare

Olivito, Renato Sante

Olmedilla, Aurelio

Olmo, Alberto

Olmo, Gabriella

Olowinsky, Alexander

Olsen, Marianne

Olšiak, Róbert

Olson, Michael W.

Olsson, Rickard

Olszewska, Aneta

Olszewski, Artur

Olteanu, Madalina

Oludemi, Taofiq

Oluwadare, Oluwatosin

Oluyisola, Olumide

Omar, Faye

Omar, Nibouche

Ombres, Luciano

Omelchenko, Denis O.

Omer, Siddig Adam

Ometov, Aleksandr

Omotayo, Temitope

Omura, Yasuhisa

Oña Simbaña, Edwin Daniel

Onac, Petroniu

Onaka, Susumu

Onas, Adrian S.

Onder, Christopher

Ondrej, Krejcar

Onel, Buket

Onesto, Luca

Onishi, Viviani

Ono, Atsushi

Ono, Kanji

Onoda, Hiroshi

Onofrei, Roxana Ramona

Onofri, Fabrice R. A.

Onuki, Hiroshi

Onwude, Daniel I.

Onyshchenko, Yuliia

Oo, Thant Zin

Oo, Win Min

Ooi, Chinchun

Oosterlinck, Willem

Opazo-Vega, Alexander

Opdyke, Aaron

Operacz, Agnieszka

Operat, Agnieszka 
Opetuk, Tihomir

Opoka, Wlodzimierz

Oppel, Markus

Oppici, Luca

Opran, Constantin

Oprea, Razvan Andrei

Opris, Ioana

Oprzędkiewicz, Krzyszof

Orand, Abbas

Orasan, Constantin

Orazbayev, Bakhtiyar

Orchard, Sandra

Orcos, Lara

Orczyk, Tomasz

Ordás, David Ruano

Ordiano, Jorge Ángel González

Ordóñez, Raziel A.

Ordoudi, Stella

Ordoukhanian, Edwin

Orellana, Luis Felipe

Orešković, Jasna

Oresta, Paolo

Orfanidis, Sotiris

Orgeira-Crespo, Pedro

Orian, Laura

Orimoto, Hisako

Orlandini, Andrea

Orlando, Maurizio

Orlov, Alexei

Orlov, Yuriy

Orlova, Ekaterina

Orłowska-Kowalska, Teresa

Orlowsky, Jeanette

Orman, Lukasz

Ormianer, Zeev

Orobtchouk, Regis

Orosei, Roberto

Orosz, Tamás

Orović, Irena

Orozco-Messana, Javier

Orsavova, Jana

Orsini, Andrea

Orsini, Giovanna

Orszaghova, Jana

Ortale, Riccardo

Ortega Sarmiento, Samuel

Ortega, Alfonso

Ortega, Fernando

Ortega, Javier

Ortega, José Marcos

Ortega, Lorenzo
Ortega-Casanova, Joaquín

Ortega-Sánchez, Delfín

Ortega-Zúñiga, Carlos A.

Ortenzi, Fernando

Ortín-Obón, Marta

Ortiz Colón, Ana María

Ortiz De Zárate, José M.

Ortiz Marcos, Isabel

Ortiz, Andric

Ortiz, Coral

Ortiz, Jordi

Ortiz, Rocío

Ortiz-Coder, Pedro

Ortuño, José María

Orudzhev, Farid

Orynycz, Olga

Orzechowski, Grzegorz

Orzelska-Górka, Jolanta

Osakada, Yasuko

Osán, János A.

Osborn, Wendy

Osei-Agyemang, Eric

Oses, Corey

Oshtorjani, Ehsan Kiani

Osipov, Andrey

Osipowicz, Tomasz

Oskarbski, Jacek

Oskiera, Michal

Osman, Shazali

Osmankovic, Dinko

Osmanlic, Fuad

Osório, Jonas H.

Osorio, Juan Pablo

Ospina, Juan

Osseiran, Adam

Ossowicz, Paula

Oßwald, Kai

Ostadhassan, Mehdi

Ostapiuk, Monika

Ostasevicius, Vytautas

Oster, Kamil

Østergaard, Martin B.

Ostoja-Starzewski, Martin

Ostos, Francisco José

Ostrovskii, Valerii

Ostrowska, Ksenia

Ostrowski, Krzysztof

Ostrowski, Piotr

Ostrowski, Rafał

Osuch, Andrzej

Oswald-Tranta, Beate 
Osypenko, Artem

Oszako, Tomasz

Oszust, Mariusz

Oszutowska-Mazurek, Dorota

Ota, Jun

Ota, Nobutoshi

Otani, Tomohiro

Otebolaku, Abayomi

Oteri, Giacomo

Otero, Andres

Otero, Jorge

Otero, Pablo

Otero, Vanessa

Otero-Espinar, Francisco Javier

Otero-Mateo, Manuel

Othman, Eman M.

Otis, Martin

Otmačić Ćurković, Helena

Otnes, Roald

Otomański, Przemysław

Otremba, Zbigniew

Otsuka, Keisuke

Otsuki, Bungo

Ott, Maria

Ottaviani, Carlo

Otten, Dale

Ottesen, Vegar

Otto, Andreas

Otto, Philipp

Otto, Tauno

Ottogalli, Kiara

Ottolenghi, Livia

Otwinowski, Henryk

Ou Yang, Hung-Yen

$\mathrm{Ou}, \mathrm{Ge}$ (Gaby)

Ouahabi, Abdeldjalil

Oubaha, Mohamed

Ouchi, Takehito

Oudot, Benoit

Ouerdane, Henni

Ouf, François-Xavier

Ougolnitsky, Guennady

Ouhyoung, Ming

Oumi, Yasunori

Oust, Are

Ouvrard, Aimeric

Ouyang, Xiaolong

Ouyang, Yingkai

Ovseník, Luboš

Ovsenik, Maja

Owen, Robert
Owerko, Tomasz

Owino, Simon Odera

Owsiński, Robert

Oyarbide, Mikel

Oyekan, John

Oyoshi, Takanori

Ozawa, Akira

Özcan, Mutlu

Ozdagli, Ali

Ozer, Ekin

Ozevin, Didem

Ozgokman, Tamay

Ozhmegov, Kirill

Ozkan-Aydin, Yasemin

Ozkaya-Ahmadov, Tevhide

Oztan, Cagri

Ozturk, Yusuf

Paar, Dalibor

Paar, Rinaldo

Pabst, Andreas Max

Pabst, Oliver

Pacaiova, Hana

Pacella, Massimo

Pach, Grzegorz

Pachta, Vasiliki

Paci, Agnese

Paci, Maurizio

Pacifici, Andrea

Pacifici, Luciano

Pacifico, Severina

Paciolla, Costantino

Paciorek-Sadowska, Joanna

Pacitto, Antonio

Pacławski, Adam

Paço, Maria

Păcularu-Burada, Bogdan

Pacurar, Razvan Ioan

Pączka, Grzegorz

Padilla, Javier

Padmanaban, Sanjeevikumar

Padmanabhan, Jagannath

Padnya, Pavel

Padovano, Antonio

Padrão, Jorge

Padrela, Luis

Pae, Eung-Kwon

Paek, Minkyu

Pagán, Esther

Paganelli, Filippo

Pagano, Stefano

Page, Jonathan 
Page, Wyatt

Pagger, Ernst

Pagiatakis, Gerasimos

Pagliarini, Giorgio

Pagliaroli, Alessandro

Pagliarulo, Vito

Pagnotta, Stefano

Pagnotti, Gabriel M.

Pagnozzi, Alex

Pagone, Emanuele

Paheding, Sidike

Pahwa, Anil

Pahwa, Roma

Pai, Kai Jen

Pai, Ming-Chang

Pai, Tun-Wen

Paiano, Roberto

Paidi, Vijay

Paik, Taejong

Paik, Younkee

Paillat, Thierry

Paindavoine, Michel

Paio, Alexandra

Pais, Sebastião

Paiva, Ana Paula

Paixão Cansado, Isabel Pestana

Paja, Wieslaw

Pajak, Beata

Pająk, Michał

Pajaziti, Arbnor

Pajic, Bojan

Pak, Alexey

Pak, Chanho

Pak, Soojong

Pakhomov, Maksim

Pakhomova, Svetlana

Pakrashi, Vikram

Pal Choudhuri, Shreoshi

Pal, Anabik

Pal, Anirban

Pal, Nabamita

Pal, Pinaki

Pal, Sudipto

Palacio, Laura

Palacz, Magdalena

Palade, Andrei

Palade, Laurentiu Mihai

Palani, Hari

Palano, Fania

Palazoglu, Ahmet

Palazon, Francisco
Palazzo, Pierfrancesco

Palchoudhury, Soubantika

Palcut, Marián

Palczynska, Beata

Paleari, Marco

Palella, Boris

Paleologu, Constantin

Palermo, Carmen

Palermo, Michele

Paleu, Viorel

Palevicius, Arvydas

Paliaga, Guido

Pałka, Norbert

Pałka, Piotr

Palkowski, Lukasz

Pall, Emoke

Palladino, Domenico

Pallandre, Antoine

Pallares, Anne

Palleschi, Vince

Palli, Gianluca

Pallottini, Valentina

Pallottino, Federico

Palma, Gianpaolo

Palma, Giorgio

Palma, Lorenzo

Palma, Miguel

Palma, Paulo

Palma, Roberto

Palma, Susana

Palmanshofer, Alexander

Palmerini, Giovanni

Palmerius, Karljohan Lundin

Palmieri, Francesco A. N.

Palmitessa, Onofrio Davide

Palo, Emma

Palomar, Teresa

Palomba, Ilaria

Palombi, Lorenzo

Palombini, Augusto

Palomo-Duarte, Manuel

Palou, Martin

Paltanea, Gheorghe

Paltinean, Ramona

Palu, Ivo

Pałubicki, Bartosz

Paluch, Andrew S.

Palumbo, Davide

Palumbo, Gaetano

Palumbo, Oriele

Palus, Katarzyna 
Pályi, Béla

Palys, Barbara

Pamart, Anthony

Pamies, Ramon

Pamuković, Jelena Kilić

Pamuła, Teresa

Pan, Baoxiang

Pan, Cuiping

Pan, Hong

Pan, Hongying

Pan, Hung-Chuan

Pan, Jeng-Shyang

Pan, Jonathan

Pan, Lee-Yun

Pan, Pengmin

Pan, Shaowu (Shawn)

Pan, Yaming

Pan, Yanbo

Pan, Yang

Pan, Yen-Liang

Pan, Yushan

Pan, Yuxin

Panagiotakis, Costas

Panagiotis, Tsarouhas

Panagiotou, Pericles

Panagopoulos, Dimitris J.

Panapakidis, Ioannis

Panaras, Giorgos

Panarotto, Massimo

Panayiotakis, George

Panayiotis, Koutsabasis

Panchenko, Vladimir

Pańcikiewicz, Krzysztof

Panda, Biranchi

Pandarese, Giuseppe

Pandelidis, Demis

Pander, Tomasz

Pandey, Anupam

Pandey, Om Jee

Pandiev, Ivailo

Pandit, Santosh

Pandíţ, Anúp

Pandya, Abhilash

Pane, Katia

Panecki, Tomasz

Paneiro, Gustavo

Panek, Jiri

Panek, Rafal

Panero, Elisa

Panero, Marco

Panfil, Wawrzyniec
Pang, Bo

Pang, Da-Chen

Pang, Lee San Natalie

Pang, Toh Yen

Pang, Xiaodan

Pani, Danilo

Panić, Branislav

Panich, Alexander M.

Panidi, Ioli

Panin, Sergey

Panitsa, Maria

Panizio, Roberta M.

Panjan, Peter

Pankowska, Malgorzata

Pannella, Gianfranco

Pannone, Daniele

Panoiu, Manuela

Panoutsakopoulos, Vassilios

Panowicz, Robert

Pantaleo, Antonella

Pantaleo, Gianni

Pantano, Antonio

Pantazis, Dimos

Pantazopoulos, George

Pantelidis, Lysandros

Panthi, Krishna

Pantoş, Dan

Pantuso, Antonio

Panwar, Mayank

Panzarasa, Guido

Pao, Tsang-Long

Paoletta, Marco

Paolini, Christopher

Paolini, Luca

Paolini, Riccardo

Paolo, Civiero

Paolucci, Federico

Pap, Bálint

Pap, László

Pap, Zsuzsa B.

Papa, Umberto

Papacek, Stepan

Papacharalampopoulos, Alexios

Papadaki, Dimitra

Papadakis, George

Papadakis, Nikolaos M.

Papadakis, Raffaello

Papadimitriou, Sofia A.

Papadopoulos, Antonios N.

Papadopoulos, Argyrios

Papadopoulos, Basil 
Papadopoulos, Christos

Papadopoulos, Gerassimos A

Papadopoulos, Nikos

Papadopoulos, Perikles

Papadopoulou, Alexandra

Papageorgas, Panagiotis

Papagiannopoulos, George

Papahristou, Evridiki

Papaioannou, Emmanouil

Papakonstantinou, Apostolos

Papakostas, George A.

Papale, Maria

Papalia, Rocco

Papalitsas, Christos

Papalou, Angeliki

Papán, Daniel

Papana, Angeliki

Papangelo, Antonio

Papanikolaou, Ioannis

Papanikou, Maria

Papari, Behnaz

Papastefanopoulos, Vasilis

Papastergiou, Maria

Papatheodorou, Efi

Papavasileiou, Georgios S.

Papia, Evaggelia

Papoutsis, Konstantinos

Pappas, Christos

Paprocka, Iwona

Paprocka, Justyna

Paprocki, Kazimierz

Papurello, Davide

Par, Matej

Paradas, Edmundo Guerra

Parajuli, Saroj

Parande, Gururaj

Paraoanu, Gheorghe

Paravastu, Narasimha

Parchami, Mohsen

Pardo Picazo, Miguel Ángel

Pardo, Laura

Pardo, Lorena

Pardo, Rebeca

Pardo-Vicente, Miguel-Ángel

Paredes, Angel

Paredes, Manuel

Parenago, Oleg P.

Parent, Gilles

Pareschi, Remo

Parfenov, Vadim

Pargar, Farhad
Pari, Luig

Paria, Debadrita

Parigger, Christian

Parinov, Ivan A.

Paris, Enrico

Parisse, Jean-Denis

Park, Bosoon

Park, Byung-Dae

Park, Byungkwon

Park, Chan-Gi

Park, Changjoo

Park, Chansik

Park, Chan-Sik

Park, Cheol Hoon

Park, Cheol Young

Park, Chul-ho

Park, Chulhwan

Park, Chung Hyuk

Park, Chun-Su

Park, Daeryong

Park, Deok-Yong

Park, Eun Soo

Park, Eunil

Park, Gyungsoon

Park, Hanhoon

Park, Heun

Park, Hong-Seok

Park, Hooncheol

Park, Ill-Woo

Park, Jaehwa

Park, Jae-Hyeung

Park, Jaehyun

Park, Jae-Sang

Park, Jaeyoung

Park, Jee Woong

Park, Ji Hwan

Park, Jihong

Park, Ji-Man

Park, Jin Yong

Park, Jinhyoung

Park, Jong-Il

Park, Jong-Sub

Park, Joontaek

Park, Juhyun

Park, Junhong

Park, Juyoun

Park, Ki-Woong

Park, Kwangsuk

Park, Kyoung Sik

Park, Kyoung Youl

Park, Kyungtae 
Park, Myoung Ryoul

Park, Nam Kyu

Park, Sangki

Park, Sehyun

Park, Seongbae

Park, Seong-Jik

Park, Seung-Bo

Park, Solmoi

Park, Suhyun

Park, Sunho

Park, Yaewon

Park, Yong Shin

Park, Yongbae

Park, YongKeun

Park, Yoo Min

Park, Young-Bum

Parkar, Shanthi G.

Parker, James

Parlatescu, Ioanina

Parlea, Lorena

Parmar, Nimai

Parmar, Paritosh

Parmet, Yisrael

Parr, David

Parra Boronat, Lorena

Parra Santiago, Jose Ignacio

Parra, Enrique

Parra, Lorena

Parrein, Benoit

Parrikar, Prathmesh Naik

Parsa, Alireza B.

Parsaeian, Shahnaz

Parsazadeh, Mohammad

Parsons, Jason

Partanen, Mikko

Parus, Arkadiusz

Parv, Luminita

Parvez, Imtiaz

Parzentny, Henryk R.

Parziale, Antonio

Parzuchowski, Paweł

Pascal, Carlos

Pascale, Francesco

Pascale, Rosa

Pascarella, Domenico

Pascual, Jose Antonio

Pascual, Juan Pablo

Pasculli, Antonio

Pashikanti, Srinath

Pashkin, Oleksiy

Pašić, Borivoje
Pasik-Duncan, Bozenna

Paskaranandavadivel, Nira

Pasławska, Marta

Pasley, Heather R

Pasqualotto, Roberto

Pasquier, Louis-César

Passarella, Daniele

Passarelli, Pier Carmine

Passian, Ali

Pasta, Salvatore

Pastor Vargas, Rafael

Pastor, Maria Magdalena

Pastore, Gianni

Pastore, Nicola

Pastorek, Filip

Pastorello, Davide

Pastor-López, Iker

Pastusiak, Tadeusz

Pastuszak, Grzegorz

Paszkiel, Szczepan

Paszkiewicz, Andrzej

Paszkowski, Robert

Páta, Petr

Patalas-Maliszewska, Justyna

Patarau, Toma

Paté, Arthur

Pateiro, Mirian

Patel, Dilip R.

Patel, Harshkumar

Patel, Jay

Patel, Kapil

Patel, Saurin

Patella, Domenico

Patelli, Edoardo

Patelski, Piotr

Pater, Sebastian

Pater, Zbigniew

Paternain, Daniel

Paterson, Kevin

Pathak, Ashish

Pathak, Rajesh

Pathakoti, Kavitha

Patience, Gregory

Patil, Ajay Bhagwan

Patil, Anuja

Patil, Ashok Kumar

Patil, Madhav

Patil, Sachin

Patil, Santosh S.

PATIL, SHRADHA

Patil, Ujwalkumar 
Patini, Romeo

Patino, Luis

Patiño, María José Martínez

Patnaik, Sourav S.

Patočka, Zdeněk

Patra, Sandipan

Pătrașcu, Monica

Patriarca, Riccardo

Patriat, Remi

Patricia, Concheiro-Moscoso

Patrício, João

Patricio, Jorge

Patrizi, Barbara

Patrone, Carlotta

Patruno, Cosimo

Pătru-Stupariu, Ileana

Patryk, Kot

Patsali, Petros

Pattammattel, Ajith

Pattanaik, Himansu

Patterson, Albert

Patterson, Jennifer

Patwary, Sarif

Pau, Giovanni

Pauchard, Ludovic

Paudel, Prabesh

Paudel, Raj

Paudyal, Dev Raj

Pauk, Jolanta

Paul, Bere

Paul, Partha P.

Paul, Sanjoy

Paul, Satyam

Paul, Seema

Paul, Shuva

Paul, Subhash

Paula, Anabela Baptista

Paula, Correia

Paulauskaite-Taraseviciene, Agne

Paulette, Laura

Pauliac-Vaujour, Emmanuelle

Pauliková, Alena

Paulino, Mariana

Paulish, Andrey Georgievich

Pauliukaite, Rasa

Paulo Leitao, Joao

Paulo Mirasol, Esther

Paulo, Carlos

Paulo, Joao Ruivo

Paulsen, Christian Oen

Paulsen, Marvin R.
Paunoiu, Viorel

Pau-Roblot, Corinne

Pauwels, Ruben

Pavanello, Fabio

Pavanello, Sofia

Pavasović, Marko

Paverd, Catherine

Pavičić, Ivica

Pavlidis, Ioannis

Pavlopoulos, Ioannis

Pavlov, Igor S.

Pavlov, Ilya N.

Pavlov, Nikolay

Pavlovic, Ana

Pavlovic, Marko

Pavlovic, Natasa

Pavlovich, Pljonkin Anton

Pavlovskis, Miroslavas

Pavlu, Dagmar

Pavlyuchenko, Evgeniy

Pavlyuk, Dmitry

Pavón Pulido, Nieves

Pawashe, Chytra

Paweł, Dymora

Pawełczyk, Anna

Pawelczyk, Marek

Pawelek, John

Pawlak, Andrzej

Pawlak, Mariusz

Pawlak, Ryszard

Pawlak-Kruczek, Halina

Pawliczek, Roland

Pawlik, Marzena

Pawlik, Pawel

Pawlis, Alexander

Pawłowicz, Bartosz

Pawłowska, Agnieszka

Pawlowski, Piotr

Pawlowski, Tadeusz

Pawlus, Paweł

Pawluszek, Kamila

Pawłyszyn, Irena

Pawyta, Miroslawa

Payá, Luis

Paz Ferreiro, Jorge

Paz, Igor

Pazera, Marcin

Paz-García, Juan Manuel

Peace, Alexander

Peake, Nicholas

Peale, Robert 
Peana, Massimiliano F.

Peaudecerf, Bruno

Peces, Miriam

Pech, Martin

Peckhaus, Andreas

Pecoraro, Giovanni

Pecorella, Tommaso

Pecori, Riccardo

Pecunia, Vincenzo

Pęczkowski, Grzegorz

Pedan, Vasilisa

Pedersen, Henrik

Pedersen, Kim B.

Pedreño Rojas, Manuel Alejandro

Pedrero González, Antonio

PEDRIELLI, Francesca

Pedrocchi, Nicola

Pedzinski, Tomasz

Pegg, Elise

Peham, Christian

Pehlken, Alexandra

Peidro, Adrian

Peinado, Alberto

Peissig, Jürgen

Pejaś, Jerzy

Pejic Bach, Mirjana

Pekar, Libor

Peláez, José A.

Peláez-Moreno, Carmen

Pelagalli, Alessandra

Pelc, Mariusz

Peleato, Borja

Peleg, Smadar

Pelicano, Christian Mark

Pelissier, Serge

Pelivan, Ivica

Pelivanov, Ivan

Pellas, Nikolaos

Pellegrini, Alessandro

Pellegrini, Marco

Pellegrini, Marika

Pellegrini, Nicola

Pellegrini, Riccardo

Pellegrino, Gerardo

Pellegrino, Maria Angela

Pellegrino, Roberta

Pellejero, Ismael

Pelli, Stefano

Pelliccioni, Paolo

Pellicer, Teresa

Pellone, Lorenzo
Peloso, Riccardo

Pelt, Daniël M.

Peltomaa, Elina

Pelton, Joseph N.

Peluso, Ilaria

Pelz, Jeff B.

Peña Pitarch, Esteban

Peña, Javier

Peña, Jose Ignacio

Peña-García, Antonio

Penava, Davorin

Penchev, Pavel

Penders, Jacques

Pendu, Mikael Le

Peng, Chang

Peng, Chao-Chung

Peng, Chun-Cheng

Peng, Cui

Peng, Jian

Peng, Ming-Tsan

Peng, Qingjin

Peng, Tongrui

Peng, Zhili

Pengo, Paolo

Penn, Gregory

Penna, Nadia

Penniston, Thomas

Pennone, Vincenzo

Pensabene, Virginia

Penson, Peter

Penzlin, Bernhard

Peoples, Cathryn

Pepe, Antonio

Pepe, Giovanni

Pepe, Massimiliano

Peppas, Georgios

Peral, Jesus

Perale, Giuseppe

Peralta, Laura

Peralta, M. Estela

Peranic, Josip

Percival, Susan S.

Perdoch, Waldemar

Perduková, Daniela

Perea Moreno, Alberto Jesús

Perea-Moreno, Miguel-Angel

Perec, Andrzej

Pereira Monteiro, Paulo

Pereira, Adelino

Pereira, André

Pereira, António M. H. 
Pereira, Jorge

Pereira, Leonel

Pereira, Luis Santos

Pereira, Octavio

Pereira, Pedro F.

Pereira, Rita

Pereira, Ruben

Pereira, Rute

Perelomov, Leonid V.

Peres, David

Peres, Emanuel

Peres, S. Camille

Perestrelo, Rosa

Pérez Arribas, Francisco

Pérez De Prado, Rocío Josefina

Pérez González, Elena María

Pérez Invernón, Francisco Javier

Pérez Martín, Rosa Ana

Pérez Menéndez, Ramón José

Perez Neira, David

Perez Salgado, Francisca

Pérez Ubeda, Rodrigo

Pérez, Agustın-Agüera

Pérez, Alfredo J.

Perez, Felipe

Pérez, José M.

Perez, Mauricio David

Pérez, Rocío

Perez-Acebo, Heriberto

Perez-Alfaro, Irene

Pérez-Álvarez, Jose Angel

Pérez-Álvarez, Leyre

Pérez-Badia, Rosa

Pérez-Blanco, Carlos Dionisio

Perez-Collazo, Carlos

Perez-Cortes, Juan-Carlos

Perez-Cruz, Angel

Pérez-Delgado, María-Luisa

Pérez-Fernández, María

Pérez-Ferreirós, Alexandra

Pérez-Hernández, Marco

Pérez-Martín, Enrique

Perez-Morago, Hector

Pérez-Sánchez, Modesto

Pérez-Soriano, Eva M.

Perez-Uribe, Andres

Pérez-Verona, Isabel Cristina

Périgo, Elio Alberto

Perikos, Isidoros

Perin, Giorgio

Perini, Roberto
Peris, Alfredo

Perişoară, Lucian Andrei

Peristeri, Eleni

Peritore, Alessio Filippo

Periwal, Priyanka

Perkins, Greg

Perko, Janez

Perković, Dario

Perlikowski, Przemysław

Permeh, Samanbar

Perna, Giuseppe

Pernaa, Johannes

Pernak, Juliusz

Pernau, Hans Fridtjof

Perne, Matija

Pernyeszi, Tímea

Peron, Fabio

Peron, Gregorio

Perono Cacciafoco, Francesco

Peroš, Kristina

Perotti, Luigi

Perpetuini, David

Perra, Cristian

Perrella, Michele

Perriman, Diana

Perrin, Lara

Perrino, Enrico

Perrone, Diego

Perrotti, Vittoria

Perrrin, Stephane

Perry, Stuart

Perš, Janez

Persijn, Stefan

Personal, Enrique

Persson, Karl-Magnus

Pertegal-Felices, María Luisa

Pertile, Marco

Perucchi, Andrea

Peruzzi, Andrea

Pervaiz, Salman

Pervej Jahan, Muhammad

Pervizpour, Mesut

Perzyk, Marcin

Pesatori, Alessandro

Pescaru, Dan

Pesch, Alexander H.

Pesek, Matevž

Pessot, Elena

Pęszor, Damian

Petelska, Aneta

Peter Amalathas, Amalraj 
Peter, Ildiko

Peterka, Jozef

Peternier, Achille

Peters, Brady

Peters, Dennis K.

Peters, William K.

Petersen, Jan

Peterson, Andrew F

Peterson, Eric Charles

Peterson, Lisa

Peterson, Rorik

Peterson-Hiller, Amie

Pethő, Zoltán

Petković, Tomislav

Petošić, Antonio

Petoumenou, Despoina

Petracchini, Francesco

Petrakis, Eleftherios

Petrakli, Fotini

PETRARIU, Adrian

Petrashova, Dina

Petrauskiene, Sandra

Petrellis, Nikos

Petrescu, Maria

Petretto, Donatella Rita

Petri, Cristian

Petrica, João

Petrich, Jan

Petriev, Iliya

Petrillo, Alberto

Petrillo, Antonella

Petrina, Tudor

Petrini, Morena

Pétritis, Dimitri

Petritoli, Enrico

Petrochenkov, Anton

Petroni, Filippo

Petrosino, Maria

Petrosyan, Arakel

Petrosyan, David

Petroutsatou, Kleopatra

Petrov, Dmitry

Petrov, Victor

Petrovčič, Simon

Petrović, Emina Kristina

Petrovič, František

Petrović, Milica

Petrovski, Steve

Petrovski, Željko

Petru, Jana

Petru, Livinti
Petru, Michal

Petrucci, Elisabetta

Petrulionis, Marius

Petrunin, Ivan

Petsa, Elli

Petsagkourakis, Postdoc Ioannis

Peyman, Azadeh

Peyman, Razi

Pezzani, Raffaele

Pezzetti, Marco

Pezzolato, Marzia

Pfeffer, Peter E.

Pfeiffer, Birgit

Pfeiffer, David C.

Pfitzner, Emanuel

Pfleger, Jiri

Pförringer, Dominik

Pfost, Heiner

Pham, Chuan

Pham, Chuyen Van

Pham, Duc Truong

Pham, Giao

Pham, Minh-Tan

Pham, Nam Thanh

Pham, Ngoc Thanh

Pham, Phuong

Pham, Quoc Bao

Pham, Quoc-Viet

Pham, Thang

Phan, Hoang Nam

Phan, Hoang Vu

Phelps, Alan

Philibert, Danielle

Philipp, Manfred

Philippot, Gilles

Philips, Neena

Phillip, Jude

Phillips, Gavin

Phillips, Jonathan

Phillips, Joshua

Phillips, Kevin

Phipps, Alex

Phoon, Kok Kwang

Phung, Luong-Viet

Phung, Quoc Tri

Phyo, Cho Nilar

Piacentini, Daniela

Piacentini, Maria Francesca

Piana, Edoardo

Piana, Michele

Piancastelli, Luca 
Piancino, Maria

Piano, Elena

Piano, Samanta

Piantini, Simone

Piasecki, Lucila

Piasta, Wojciech

Piattelli, Adriano

Pibernik, Jesenka

Pica, Monica

Picado-Santos, Luis

Piccinini, Leonardo

Piccinni, Giovanni

Piccioni, Cristiana

Piccirillo, Clara

Picerno, Pietro

Pichler, Anita

Pichór, Waldemar

Piciarelli, Claudio

Pickard, Matt

Pickford, Russell

Picó Vila, Rubén

Picollo, Federico

Pié, Laia

Piechota, Katarzyna

Piedade, João

Piegat, Agnieszka

Piekarczyk, Marcin

Piekoszewski, Wojciech

Piemonte, Andrea

Piemontese, Luca

Pieper, Jeff

Piercey-Normore, Michele

Pierdicca, Roberto

Piergies, Natalia

Pierleoni, Paola

Pierpaoli, Mattia

Pierre, Siron

Pierściński, Kamil

Pietrasik, Joanna

Pietraszek, Jacek

Pietrelli, Loris

Pietriková, Emília

Pietrowicz, Slawomir

Pietruschka, Dirk

Pietrusewicz, Krzysztof

Pietrusiak, Damian

Pietruszewska, Anna

Pietrzak, Krystian

Pietrzak, Piotr

Pietsch, Ullrich

Pievani, Alice
Pigłowski, Marcin

Pihut, Malgorzata

Pijls, Bart

Pikhay, Evgeny

Pikoulis, Erion-Vasilis

Pikridas, Michael

Pikulin, Alexander

Pilar González Vázquez, María

Pilarska, Agnieszka

PILAT, Adam Krzysztof

Pilate, Pascal

Pilato, Giovanni

Pilecka, Elżbieta

Pilecki, Zenon

Pileggi, Salvatore F.

Pilehvar, Shima

Pilipović, Ana

Pilmane, Māra

Pilo, Raphael

Piltan, Farzin

Pina Gadea, Carmelo

Piña Ramirez, Carolina

Pinardi, Stefano

Pina-Vaz, Irene

Pincheira, Patricio A.

Pinchera, Daniele

Pineau, Pascal

Pineda, Miguel

Pineda, Paloma

Pineda-Sanchez, Manuel

Pinela, José

Piñerez Torrijos, Iván Darío

Piñero, Jose Carlos

Pinheiro, Jorge

Pinheiro, Manuel Duarte

Pinheiro, Mario

Pinho, Carlos

Pinho, Francisco

Pinho, Henrique J. O.

Pinho, José

Pinho, Luís

Pinho, Marcelo

Pinho, Pedro

Pinho, Tatiana M.

Pini, Gian Andrea

Pinilla, Javier

Pinion, Clint

Pinon Pereira Dias, Joao Victor

Pinson, Jean

Pintea, Camelia M.

Pinter, Gabor 
Pinto, Deesy

Pinto, Gustavo

Pinto, Gustavo Filipe

Pinto, Loris

Pinto, Maria Isabel Moita

Pinto, Mónica

Pinto, Pedro

Pinto, Sandra

Pinto, Sonia Ferreira

Pinto, Teresa

Pintore, Giovanni

Pintus, Ruggero

Piorecky, Marek

Piórkowska, Katarzyna

Piorkowski, Adam

Piot-Lepetit, Isabelle

Piotr Lipiński, Piotr

Piotr, Lichota

Piotrowska, Katarzyna

Piotrowska, Małgorzata

Piotrowski, Paulina

Piotrowski, Paweł

Piotrowski, Tomasz

Pipino, Caterina

Pipintakos, Georgios

Piqueras, Pedro

Piracha, Afaq

Pirandola, Stefano

Pirani, Vittorio

Piraveenan, Mahendra

Pires De Lima, Rafael

Pires, Gabriel

Pires, Ivan Miguel Serrano

Pires, Layla

Pires, Luis Carlos Carvalho

Pires, Vitor Fernão

Pirnia, Mehrdad

Pirouz, Behrouz

Pirouz, Matin

Pirozhkov, Alexander S.

Pisapia, Pasquale

Pisarcik, Martin

Pisaturo, Giuseppe Roberto

Piscitelli, Filomena

Piselli, Cristina

Pisello, Anna

Pishchalnikov, Roman Y.

Pisk, Jana

Piskin, Senol

Piskorski, Łukasz

Piskunov, Maxim
Pisla, Doina

Pislaru, Marius

Pisoni, Galena

Píštěk, Václav

Pisz, Iwona

Pitacco, Andrea

Pitarch, Aida

Pitarch, Jose Luis

Pitas, Charalampos N.

Pitel', Ján

Pitla, Santosh

Pitre, Frédéric

Pitropakis, Nick

Pitt, Jonathan S.

Pitti, Rostand Moutou

Pitto, Andrea

Pitts, Teresa

Piunti, Andrea

Piva, Mariacristina

Piwowar, Arkadiusz

Piwowarski, Marian

Piwowarski, Mateusz

Pizzanelli, Silvia

Pizzi, Antonio

Pizzoferrato, Roberto

Pizzolante, Raffaele

Pizzoli, Silvia Francesca Maria

Pizzuti, Clara

Płaczek, Bartłomiej

Planat, Michel

Plasenzotti, Roberto

Plass-Oude Bos, Danny

Plastina, Pierluigi

Platek, Pawel

Platero, Carlos A.

Plathottam, Siby Jose

Platil, Antonin

Platoš, Jan

Plavsic, Tomislav

Plaza, Encarna Gomez

Plazzotta, Stella

Plebankiewicz, Edyta

Plechawska-Wójcik, Małgorzata

Pletl, Szilveszter

Plikusiene, Ieva Baleviciute

Plinta, Dariusz

Płotka-Wasylka, Justyna

Plotnikov, Mikhail Y.

Pluciński, Jerzy

Pluciński, Marcin

Pluháček, Michal 
Plummer, David

Plummer, Sue

Plutino, Maria Rosaria

Pluvinage, Guy A.

Pnevmatikos, Nikos

Pobar, Miran

Pochwat, Bartłomiej

Pochwat, Kamil

Pochylski, Mikolaj

Podda, Alessandro Sebastian

Podgorelec, Vili

Podgórski, Jerzy

Podgórski, Maciej

Podhorski, Adam

Podilchak, Symon K.

Podkovalnikov, Sergei

Podlaha-Murphy, Elizabeth

Podlasek, Anna

Pödör, Andrea

Podržaj, Primož

Podvorica, Fetah

Podzimek, Stepan

Poeggeler, Burkhard

Poenaru, Violeta

Poerschmann, Christoph

Pogačnik, Željko

POGULURI, SUNNY KUMAR

Poh, Hee Joo

Pohjankukka, Jonne

Pohl, Elena

Pohl, Sebastian

Pohlman, Nicholas A.

Poiana, Marco

Poiana, Mariana-Atena

Poirier-Quinot, David

Pokhrel, Lok

Pokhrel, Madhab

Pokhrel, Rama Mohan

Pokora, Ilona

Pokrovsky, Oleg S.

Pokryvkova, Jozefina

Polach, Pavel

Polaczyk, Pawel

Polak, Adam

Polanski, Marek

Polap, Dawid

Polasik, Robert

Polastri, Andrea

Polat, Emre Ozan

Polatidis, Nikolaos

Polechońska, Ludmiła
Polechoński, Jacek

Poletti, Giorgio

Poletto, Luca

Poli, Davide

Policarpos, Papadopoulos

Polinski, Jaroslaw

Politis, Archontis

Polito, Laura

Pollari, Francesco

Pollicino, Antonino

Pollman, Anthony G.

Pöllmann, Herbert

Pollmann, Katrin

Pollner, Peter

Polly, David

Polowczyk, Izabela

Polrolniczak, Edward

Poltorak, Lukasz

Poltronieri, Filippo

Poltschak, Florian

Polyakov, Roman

Polykretis, Christos

Pomarede, Pascal

POMARES TORRES, JUAN CARLOS

Pomastowski, Paweł P.

Pombo, Iñigo

Pompurová, Kristína

Poncet, Sébastien

Ponciano, Jean-Jacques

Poniedziałek, Barbara

Poniewiera, Marian

Ponikiewski, Tomasz

Ponn, Thomas

Ponomarev, Andrew

Pons, Alexander

Pons, Dirk

Pons, Josefina

Pons, Oriol

Pons, Ramon

Ponsa, Pere

Pont, Ulrich

Pont, Ulrich J.

Ponta, Linda

Ponte, Salvatore

Ponti, Cristina

Ponticelli, Gennaro Salvatore

Ponticorvo, Michela

Pontius, Frederick W.

Ponzetto, Antonio

Poór, Péter

Pooranian, Zahra 
Poorjam, Amir Hossein

Poovvancheri, Jiju

Pop, Adrian

Pop, Claudia Violeta

Pop, Flavia

Pop, Horia F.

Pop, Izabela Luiza

Pop, Mihai Alin

Popa, Dan-Cristian

Popa, Daniel

Popa, Daniela-Saveta

Popa, Mona Elena

Popek, Zbigniew

Popelka, Stanislav

Popenda, Andrzej

Popescu, Andrei

Popescu, Dan

Popescu, Daniela-Elena

Popescu, Diana

Popescu, Dorin

Popescu, Ionel Catalin

Popescu, Mihaela R.

Popescu, Theodor D.

Popescu, Vlad

Popko, Ewa

Popkov, Yuri S.

Poplawski, Tomasz

Popli, Nipun

Popoff, Steven

Popov, Anton

Popov, Dimityr

Popova-Gorelova, Daria

Popović Đorđević, Jelena

Popovic, Marko

Popowczak, Marek

Popowicz, Adam

Porada, Christopher D.

Porowski, Adam

Porras Soriano, Rocío

Porrelli, Davide

Porru, Mario

Portela, Filipe

Portesi, Chiara

Portilla, Jorge

Porto, Simona

Portugal, Antonio

Portugal, Paulo

Porumb, Radu

Porwik, Piotr

Porzio, Alberto

Posada-Baquero, Rosa
Posada-Quintero, Hugo F.

Posadas, Pilar

Poskus, Tomas

Pospiech, Doris

Postacchini, Matteo

Posteraro, Federico

Potapov, Vadim V.

Potenza, Francesco

Potenziani, Marco

Potha, Nektaria

Pothupitiya, Jinal

POTIRAKIS, Stelios

Potočnik, Igor

Potok, Zbigniew

Potolea, Rodica

Potter, Mark

Pottier, Bernard

Pougoum, Fabrice

Pouliakis, Abraham

Pouliaris, Christos

Poulikakos, Lily

Pouliquen, Mathieu

Poulopoulos, Panos

Poulopoulos, Vassilis

Poulová, Petra

Poulsen, Rene Taudal

Pour, Amin Beiranvand

Pourakbari Kasmaei, Mahdi

Pouranian, M. Reza

Pourbaghi Masouleh, Milad

Pourhejazy, Pourya

Pourrezaei, Kambiz

Poveda-Martínez, Pedro

Povh, Teja Koler

Powell, Folami Lamoke

Powell, Tommy

Powers, James

Powrie, Honor

Poza-Lujan, Jose-Luis

Pozo, Borja

Pozorski, Zbigniew

Pozzebon, Alessandro

Pozzo, Eleonora Da

Prabakaran, Mayakrishnan

Prabel, Benoit

Prabowo, Aditya Rio

Prabowo, Briliant Adhi

Praczyk, Tomasz

Prada, Alessandro

Pradal, Delphine

Pradère, Christophe 
Prades, Joan Daniel

Pradhan, Kallol

Pradhan, Shantanu

Prado Da Fonseca, Vinicius

Prados-Privado, María

Prajzler, Vaclav

Prakash, Naveen

Prakash, Ranganathan

Prakht, Vladimir

Prakoso, Ari Bimo

Pramanik, Brahmananda

Pramudita, Jonas A.

Pranckeviciene, Erinija

Pranggono, Bernardi

Prapotnik Brdnik, Anita

Prasad, Dilip K.

Prasadh, Somasundaram

Prashant, Kumar

Prashar, Sanjiv

Prat, Josep

Pratap Singh, Anubhav

Pratas, José Maria

Pratt, Lawrence

Prause, Christian

Pravettoni, Mauro

Pražan, Radek

Prażnowski, Krzysztof

Prazuch, Janusz

Preda, Elena

Preda, Radu Ovidiu

Preethichandra, Daluwathu Mulla Gamage

Pręgowska, Agnieszka

Preinstorfer, Philipp

Prelipceanu, Marius

Premachandra, Chinthaka

Premier, Alessandro

Premnath, Kannan N.

Premrov, Miroslav

Presentato, Alessandro

Prestileo, Fernanda

Preston, Daniel J.

Preston, Victoria

Presutti, Livio

Presz, Wojciech

Prete, Maria Irene

Prete, Roberta

Preti, Delia

Preti, Giulio

Preusse, Axel

Preuveneers, Davy

Preziosi, Elisabetta
Priante, Davide

Přibil, Jiří

Pribilova, Anna

Pribyl, Julia

Pricop, Emil

Priego Quesada, Jose Ignacio

Prieler, Rene

Prieto Herráez, Diego

Prieto, Andrés

Prieto, Carlos

Prieto, Elena

Prieto, Jesús-Ignacio

Prieto, Mariano Sanz

Prikazchikov, Danila

Prikhodko, Valentina

Primatesta, Stefano

Primc, Gregor

Primožič, Ines

Primus, Carolyn M.

Prince, Kevin Charles

Principi, Paolo

Prins, Maarten R.

Prinzie, Jeffrey

Pritz, Balazs

Privat-Maldonado, Angela

Prlić Kardum, Jasna

Pro Muñoz, Carmen

Probst, Janice C.

Procházka, Ales

Prochazka, Ivan

Prochazkova, Jana

Procházková, Lenka

Prochazkova, Petra

Prochon, Miroslawa

Prochon, Piotr

Prochor, Piotr

Procopio, Renato

PRODANOVIC, Radivoje

Proença Brójo, Francisco Miguel Ribeiro

Proestos, Charalampos

Proietti, Tommaso

Prokic, Vesna

Pron, Hervé

Propst, Evan J.

Prot, Victorien

Proud, William

Proux, Olivier

Provolo, Giorgio

Provot, Thomas

Prpić, Zvonimir

Prpic-Orsic, Jasna 
Prudenzano, Francesco

Pruess, Birgit M.

Pruks, Vitalii

Pruna, Alina

Prussi, Matteo

Pryamikov, Andrey

Prywer, Jolanta

Przednowek, Krzysztof

Przestacki, Damian

Przybył, Andrzej

Przybyła-Kasperek, Małgorzata

Przybyłek, Maciej

Przybylek, Piotr

Przybylska-Balcerek, Anna

Przybysz, Arkadiusz

Przybysz-Romatowska, Marta

Przygodziński, Przemysław

Przygodzki, Tomasz

Przysowa, Radosław

Przystupa, Krzysztof

Psaila, Giuseppe

Psarobas, Ioannis

Pshenay-Severin, Dmitry A.

Psillakis, Haris E.

Psilovikos, Aris

Psomiadis, Emmanouil

Psomopoulos, Constantinos S.

Psota, Eric

Psota, Pavel

Pstrowska, Katarzyna

Psuj, Grzegorz

Psychalinos, Costas

Psyllaki, Pandora P.

Pszczoła, Marek

Ptacek, Michal

Ptáček, Petr

Ptak, Mariusz

Ptak, Szymon

Ptak-Chmielewska, Aneta

Ptashnik, Igor' V

Ptaszynski, Michal

Ptiček Siročić, Anita

$\mathrm{Pu}$, Haihui

$\mathrm{Pu}$, Yunting

Puac, Nevena

Pucci, Andrea

Pucciarelli, Giuseppe

Puchades, Ivan

Puchalka, Radoslaw

Pucher, Johannes

Puchkov, Evgeny
Pucihar, Andreja

Pučko, Zoran

Pudovikov, Sergey

Pudukotai Dinakarrao, Sai Manoj

Pudziuvelyte, Lauryna

Puebla, Ricardo

Puech, Pascal

Pueo, Basilio

Pueppke, Steven

Puerta Morales, Francisco Javier

Puertas, Antonio M.

Puértolas, Jaime

Puetzer, Jennifer

Puffer, John

Pugi, Luca

Puglisi, Donatell

Pugna, Ardian

Pugnetti, Carlo

Puigdollers, Joaquim

Puiggalí-Jou, Anna

Pujadas Álvarez, Pablo

Pujol Lopez, Francisco Antonio

Pukalchik, Mariia

Pukalskas, Saugirdas

Pukhov, Alexander

Pulavarti, Surya VSRK

Pulay, Alana

Pulidindi, Indra Neel

Pulido-Melián, Elisenda

Pulido-Moncada, Mansonia

Pulina, Pietro

Pulkkinen, Aki

Pullano, Salvatore A.

Pullen, Keith Robert

Pummer, Elena

Puna, Jaime

Punchihewa, Amal

Pung, Aaron J.

Punov, Plamen Borisov

Puppe, Daniel

Puppi, Dario

Puppio, Mario Lucio

Puranik, Tejas

Puscaselu, Roxana

Puschita, Emanuel

Pushkar, Svetlana

Pushkarev, Pavel

Puskar, Michal

Puślecki, Łukasz

Pussi, Katariina

Puszynski, Krzysztof 
Putaux, Jean-Luc

Putilov, Arcady

Putland, Rosalyn L.

Putz, Łukasz

Puziene, Ruta

Pycia, Karolina

Pyka, Alina

Pyka-Pająk, Alina

Pyl, Lincy

Pyle, David

Pyo, Sukhoon

Pyra, Józef

Pyrgaki, Konstantina

Pyrgioti, Eleftheria

Pyrzowski, Łukasz

Pytel, Vanesa

Pyun, Sukjoon

Pyzik, Adam

Qadri, Muhammad Yasir

Qarmout, Mahmoud

Qaroush, Abdussalam

Qasim, Muhammad

Qassem, Meha

Qattawi, Ala

Qi, Guanqiu

Qi, Han

Qi, Junpeng

Qi, Qian-Rong

Qi, Zheng

Qi, Zhuyuan

Qian, Chunqi

Qian, Guowei

Qian, Jin-yuan

Qian, Kun

Qian, Xuejun

Qiao, Mingyu

Qiao, Yongliang

Qiao, Yuansong

Qiaoxi, Zhu

Qin, Tong

Qin, Xulei

Qin, Yuxiao

Qiu, Bin

Qiu, Chong

Qiu, Cong

Qiu, Gang

Qiu, Hai

Qiu, Zhen

Qneibi, Mohammad

Qu, Feijun

$\mathrm{Qu}$, Jun
Quadri, Christian

Quan, Li

Quan, Nguyen Van

Quaquero, Emanuela

Quaranta, Emanuele

Quaresma, Paulo

Quarez, Eric

Quattrocchi, Antonino

Quax, Paul

Queiros, Carla

Queiros, Ricardo

Quendler, Elisabeth

Querales-Flores, Jose

Quessab, Yassine

Quest, Dale

Quevedo Pérez, Jose Ramón

Quevedo, Eduardo

Quevedo, Miguel

Quevedo-Teruel, Oscar

Quijano-Sánchez, Lara

Quindos, Luis

Quinn, Ashlinn

Quint, Wolfgang

Quintarelli, Elisa

Quintero-Bonilla, Santiago

Quinzi, Vincenzo

Quoc Le, Trung (Tim)

Qureshi, Amna

Quynh Hoa, Le

R. Smit, Menno

R. Tear, Gareth

Ra, In-ho

Raak, Norbert

Raake, Philip W. J.

Raayai, Shabnam

Rabaev, Irina

Rabah, Hassan

Rabbani, Mohammad Mahbub

Rabbani, Muhammad Saqib

Rabcan, Jan

Rabczuk, Timon

Raber-Durlacher, Judith E.

Rabiee, Ramin

Rabiee, Ramtin

Rabilloud, Thierry

Rabinovici, Raul

Race, Marco

Rachwalski, Michał

Rackiene, Roma

Rackwitz, Frank

Rácz, Richard Péter 
Rączka, Waldemar

Raczkiewicz, Wioletta

Raczkowska, Joanna

Radac, Mircea-Bogdan

Radanliev, Petar

Radescu, Radu

Radhakrishnan, Sivaprakasam

Radic, Danko

Radić, Tomislav

Radicioni, Fabio

Radin, Michael

Radkowska, Iwona

Radkowski, Rafael

Radman, Sanja

Radnor, John M.

Radoi, Radu

Radon, Jan

Radonja, Radoslav

Radu, Beatrice Mihaela

Radu, Dorin

Radu, Laura-Diana

Radu, Rosca

Raducanu, Bogdan C.

Răducanu, Doina

Radulian, Mircea

Radwan-Pragłowska, Julia

Radziemska, Maja

Radziszewska-Zielina, Elżbieta

Radzka, Elżbieta

Raeis-Hosseini, Niloufar

Rafał, Marcin Laskowski

Rafalskaya, Tatyana

Raffaele, Lorenzo

Raffaeli, Roberto

Raffaelli, Carla

Raffi, Milena

Rafieeinasab, Arezoo

Rafique, Raza

Ragauskas, Arthur J.

Raggi, Andrea

Raggiunto, Sara

Raghavan, Nagarajan

Ragos, Omiros

Ragosta, Maria

Ragusa, Edoardo

Rahaman, Hafizur

Rahbar-Rastegar, Reyhaneh

Rahimi Tabar, Mohammad Reza

Rahimi, Sohrab

Rahimi-Gorji, Mohammad

Rahimpour, Alireza
Rahm, Johannes

Rahman Rashid, Rizwan Abdul

Rahman, Akhlaqur

Rahman, Akm S.

Rahman, Ashiqur X.

Rahman, Azizur

Rahman, Hafizur

Rahman, Juber

Rahman, Md Anisur

Rahman, Md Moktadir

Rahman, Mizanoor

Rahman, Mizanur

Rahman, Mohammad Atikur

Rahman, Mohammad Mahmudur

Rahman, Mostaqur

Rahman, MuhibUr

Rahman, Muhommad Azizur

Rahman, S M Ashrafur

Rahman, Sabidur

Rahmani, Fatemeh

Rahmathulla, Gazanfar

Rahmati-Abkenar, Mahboubeh

Rahmatian, Arash

Rahnejat, Homer

Rai, Vikrant

Raibulet, Claudia

Raicopol, Matei D.

Raileanu, Silviu

Raimondo, Marialuigia

Rainero, Elena

Rainieri, Carlo

Raith, Stefan

Raj, Eldon

Raj, Nawin

Raj, Saurabh

Raja Bose, Jefferson

Raja, Rajikha

Rajabloo, Talieh

Rajagopalan, Uma Maheswari

Rajala, Pauliina

Rajamani, Kumar

Rajan, Parthiban

Rajan, Robin

Rajapakse, Athula

Rajaram, Dushhyanth

Rajaraman, Sivaramakrishnan

Rajchowski, Piotr

Rajčić, Vlatka

Rajczakowska, Magdalena

Rajda, Janusz

Rajewski, Remigiusz 
Rajput, Shailendra

Rajski, Krzysztof

Rajtukova, Viktoria

Rak, Janusz

Rakić, Dragan

Rakoczy, Rafał

Rakovich, Aliaksandra

Ralston, Jonathon

Raluca, Brehar

Ram, Rahul

Rama, Estefanía Costa

Ramaglia, Giancarlo

Ramakrishnan, Sayanthan

Ramalho-Santos, João

Ramalingam, Balakrishnan

Ramamoorthy, Sunil Kumar Lindström

Ramanauskaitè, Simona

Ramanavicius, Arunas

Ramanujan, Raju

Ramaraj, Pandurangan

Ramasamy, Elamparuthi

Ramasamy, Parthiban

Ramasamy, Thiruganesh

Rambaccussing, Dooruj

Ramella, Chiara

Ramezani, Majid G.

Ramieri, Guglielmo

Ramirez Miquet, Evelio

Ramirez, Dionisio

Ramirez, Juan Marcos

Ramirez-Castellanos, Julio

Ramirez-Castrillon, Mauricio

Ramírez-Vargas, Carlos A.

Ramiro-Cortijo, David

Ramis Soriano, Jaime

Ramón Jerónimo, María Ángeles

Ramos Gavilán, Ana Belén

Ramos González, Victoria

Ramos Miras, Jose Joaquin

Ramos Requena, José Pedro

Ramos, Ana

Ramos, António

Ramos, Delfina Gabriela Garrido

Ramos, Gabriel

Ramos, Helena M.

Ramos, João António Esteves

Ramos, Josean

Ramos, Lucía

Ramos, Victor

Ramos-Garcia, Pablo

Ramos-Vivas, José
Ramsey, Dan K.

Ramzanpour, Mohammadreza

Rana, Abu Ul Hassan Sarwar

Rana, Gianfranco

Rana, Ziaul Hasan

Ranadheera, Senaka

Ranagalage, Manjula

Ranalli, Giancarlo

Randewijk, Peter Jan

Randle, Hayley

Rangarajan, Shriram S.

Rangel, Pablo

Rangel-Hernandez, Victor

Ranieri, Vittorio

Ranjbarzefreh, Mahdi

Ranky, Paul

Ranstam, Jonas

Ranta, Ovidiu

Rantanen, Esa

Rantos, Konstantinos

Ranzato, Elia

Rao, Ashit

Rao, Posinasetti Nageswara

Rao, Qi

Rao, Sandro

Rao, Shivanesh Arvinda

Rapa, Mattia

Rapagnà, Sergio

Rapone, Biagio

Raposeiro Da Silva, Pedro

Raposo, António

Raposo, Duarte

Raposo, Mauro

Rapp, Ludovic

Rappel, Hussein

Rappelli, Giorgio

Raptis, Dimitrios

Raptis, George E.

Raptis, Ioannis-Panagiotis

Raptis, Panagiotis Ioannis

Raptis, Theofanis

Rasche, Christoph

Rashed-Ali, Hazem

Rashid, Mizanur

Rashidi, Ali

Rasmussen, Anders

Rasol, Mezgeen

Rasolofondraibe, Lanto

Rasolomampionona, Desire D.

Rass, Stefan

Rasskazov, Ilia 
Rassman, William

Rassõlkin, Anton

Rastegari, Elham

Rastelli, Eugenio

Rastelli, Gianluca

Rastogi, Anshu

Rata, Gabriela

Ratajczak, Katarzyna

Ratajczak, Monika

Ratassepp, Madis

Ratchford, Steve

Rathod, Dhruvang

Rathore, Shailendra

Ratnakumar, Bugga V.

Ratner, Svetlana

Rato, Vasco Moreira

Ratzke, Markus

Rau, Matthew J.

Rauch, Erwin

Rauh, Andreas

Raul Marin, Eng

Rault, Francois

Rauschnabel, Phil

Raush, Gustavo

Raut, Prasad

Rauwel, Erwan

Rauwel, Protima

Ravan, Maryam

Ravankar, Abhijeet

Ravat, Franck

Ravelet, Florent

Ravelo, Blaise

Ravindra, Nuggehalli M.

Ravindran, Arun

Ravula, Sudhir

Rawat, Swati

Rawski, Mariusz

Ray, Sujay

Ray, Supriyo

Raya-González, Javier

Rayguru, Madan Mohan

Raykova, Reneta

Raynie, Douglas

Razaak, Manzoor

Razack, Moumtaz

Razavi, Javad

Razban, Ali

Razenkov, Igor

Razi, Hajar

Razik, Hubert

Razkenari, Mohamad
Razmkhah, Omid

Raźniak, Piotr

Razzaghmanesh, Mostafa

Razzokov, Jamoliddin

Reali, Gianluca

Reatti, Alberto

Rebaioli, Lara

Rebedea, Traian

Rebelo, Ana

Rebelo, Carlos

Rebollo-Hernanz, Miguel

REBORDÃO, JOSÉ MANUEL

Reboud, Christophe

Reddy, Mogalahalli Venkatesh

Reddy, Sohail R.

Redel-Macías, María Dolores

Redlarski, Grzegorz

Redon, Pau

Redowicz, Maria Jolanta

Redutskiy, Yury

Reduzzi, Maurizio

Reece, Lisa M.

Reed, Kevin F.M.

Reeke, George N.

Rees, D. Andrew S.

Rees, Stephen

Reeves, Adam

Reeves, Lawrence E.

Regattieri, Alberto

Reggiani, Luca

Regiec, Andrzej

Regla-Nava, Jose A.

Regmi, Bishnu

Regmi, Madan Bandhu

Regueira, Teresa

Réh, Roman

Rehrig, Erin

Rehrl, Jakob

Reich, Adam

Reichhardt, Charles

Reid, Mark

Reifsnider, Kenneth L.

Reig, Càndid

Reiger, Matthias

Reina, Daniel Gutiérrez

Reinaldo Ribeiro, Fernando

Reina-Romo, Esther

Reinaud, Jean

Reineholm, Cathrine

Reiner, Johannes

Reiners, Torsten 
Reinholds, Ingars

Reining, Christopher

Reinosa, Julián Jiménez

Reinoso, Oscar

Reinprecht, Ladislav

Reis, Anabela

Reis, António Heitor

Reis, Gerd

Reis, Gustavo

Reis, João

Reis, Manuel J. C. S.

Reisis, Dionysios

Reisslein, Martin

Reiterer, Alexander

Reiterman, Pavel

Reithmeier, Eduard

Reja, Md Yousuf

Rékási, Márk

Rekatsinas, Christoforos S.

Relich, Marcin

Relvas, Helder

Remedios Serrano, Dolores

Remesar, Xavier

Remigante, Alessia

Remisova, Eva

Remmas, Nikolaos

Remondino, Marco

Rempel, Alexandra R.

Ren, Dingkun

Ren, Haoshan

Ren, Huilong

Ren, Liqiang

Ren, Wei

Ren, Wuwei

Ren, Yuzhuo

Ren, Zhenhua

Renard, Didier

Renard, Jean-Baptiste

Renau-Pruñonosa, Arianna

Rende, Sante Francesco

Rene, Eldon

René, Miloš

Renedo, Carlos J.

Rengarajan, Amirtharajan

Rengo, Marco

Renman, Gunno

Renner, Kristen

Rennhofer, Marcus

Renò, Filippo

Renson, Audrey

Rente, Bruno
Renwick, Sarah Louise

Renzaglia, Alessandro

Rescalvo, Francisco

Resende, Ricardo Pontes

Resnick, Andrew

Restel, Franciszek

Reta, Daniel

Rettig, Rasmus

Reul, Christian

Reuter, Tim

Reutzel, Edward

Revelou, Panagiota-Kyriaki

Revollar, Silvana

Revuelta, María Aránzazu

Rexha, Blerim

Rey, Gaëtan

Reyer, Henry

Reyes Belmonte, Miguel Ángel

Reyes Menéndez, Ana

Reyes, Encarnación

Reyes-Aldasoro, Constantino Carlos

Reyes-Cabrera, Joel

Reymond, David

Reynolds, Thomas

Rezaei Rad, Aryan

Rezaei, Asghar

Rezaeieh, Sasan Ahdi

Rezaie, Reza

Rezapour Mashhadi, Mohammad Mahdi

Rezig, Sadok

Rezk, Ahmed

Reznik, Jacqueline E.

Řezník, Tomáš

Rezvani, Ehsan

Rezvani, Mohsen

Rezvani, Seyed Ali

Rezzoug, Nasser

Rhee, Eun Joo

Rhee, Huinam

Rhee, Je-Keun

Rhee, Sang-Bong

Rheinwalt, Aljoscha

Rhi, Seok-Ho

Rhodin, Helge

Ria, Francesco

Riad, Abanoub

Riad, Khettabi

Riaz, Qaiser

Riba, Jordi-Roger

Ribakov, Yuri

Ribeiro, Apoena 


\author{
Ribeiro, Artur \\ Ribeiro, Bruno \\ Ribeiro, Eugénio \\ Ribeiro, Helena \\ Ribeiro, João \\ Ribeiro, Ricardo \\ Ribeiro, Sergio Silva \\ Ribeiro, Viviana Pinto \\ Ribes-Pleguezuelo, Pol \\ Ribickis, Leonids \\ Ribodetti, Alessandra \\ Rica Alarcón, Raúl Alberto \\ Ricardez-Sandoval, Luis \\ Riccardi, Giovanna \\ Riccardi, Umberto \\ Ricci, Alessio \\ Ricci, Arianna \\ Ricci, Daniele \\ Ricci, Martina \\ Ricci, Sara \\ Ricciardi, Giuseppe \\ Ricciardi, Vincenzo \\ Riccio, Aniello \\ Riccio, Giuseppe \\ Ricciu, Roberto \\ Riccò, Matteo \\ Richard, Melissa \\ Richards, Dylan Jack \\ Richardson, Justin \\ Richardson, Martin \\ Richardson, Robert \\ Richert, Anja \\ Richert, Maria \\ Richetta, Maria \\ Richiedei, Dario \\ Richter, Marc \\ Rickard, Matthew \\ Rida, Imad \\ Ridolfi, Alessandro \\ Rie, Dong-Ho \\ Riedl, Michael \\ Riegler, Martin \\ Riel, Andreas \\ Rienstra, Sjoerd \\ Riera, Bernard \\ Riffelmacher, Thomas \\ Rigas, Emmanouil S. \\ Rigby, Sam \\ Riggio, Mariapaola \\ Righi, Marco \\ Rigon, Daniele
}

Riguidel, Michel

Riha, Shannon

Rikhtegar, Farhad

Rikmann, Ergo

Riley, Sean

Riljak, Vladimír

Rim, Minjoong

Rimando, Jeremy

Rimár, Miroslav

Rimkus, Alfredas

Rimola Gibert, Albert

Rinaldi, Giovanni

Rinaldi, Marianna

Ringler, Adam

Rintoul, Naomi

Rios, Sara

Ríos-Santos, Jose Vicente

Riou, Laurent M.

Ripolles, Teresa S.

Riquelme, José C.

Risi, Michele

Rispoli, Concetta

Ristic, Michael

Ritari, Antti

Ritter, Helmut

Rivas, Sandra

Rivas-Blanco, Irene

Rivera, Christian A.

Rivera, Francisco Fernández

Rivera, Sergio Raul

Rivera, Zandra Betzabe

Rivera-González, Luis

Rivero, Carlos R.

Rivero, Pedro J.

Riveros, Guillermo A.

Riviera, Pier Paolo

Rivière-Lorphèvre, Edouard

Rivka, Fidel

Rizal, Conrad

Riziotis, Christos

Rizk, Elias B.

Rizman Zalik, Krista

Rizou, Zoe V.

Rizov, Victor Iliev

Rizun, Nina

Rizvi, Syed Tahir Hussain

Rizvi, Zarghaam Haider

Rizzarelli, Paola

Rizzi, Alessandro

Rizzo, Carmen

Rizzo, Enzo 
Rizzo, Fabio

Rizzo, Giovanna

Rizzo, Giuseppe

Rizzo, Valeria

Rizzolio, Flavio

Rizzuto, Joseph

Robb, Nigel D.

Robert, Frédéric

Robert, Wimpory

Roberto, Roberta

Robertovna, Garsiya Ekaterina

Roberts, Ronald

Robertson, Joseph W. F.

Robescu, Lacramioara Diana

Robinson, Peter

Robison, Jennifer

Robitzsch, Alexander

Robledo, Virginia Rodríguez

Robles, Ramiro Samano

Robles-Gómez, Antonio

Robles-Rodriguez, Carlos E.

Robusto, Francesco

Rocca, Fabio

Roccetti, Marco

Rocchi, Irene

Rocco, Davide

Roccotelli, Michele

Roccuzzo, Andrea

Rocha, Ada Margarida Correia Nunes Da

Rocha, Ana Patrícia

Rocha, Djenisa H. A.

Rocha, Fernando

Rocha, Leonel

Rocha, Mateus Garcia

Rocha, Paulo

Rochani, Ankit

Roche, Enrique

Rochette Cordeiro, António Manuel

Rochette, Martin

Ročka, Saulius

Rodehorst, Uta C.

Rodehorst, Volker

Rodella, Ilaria

Rodias, Efthymios

Rodič, Blaž

Rodil, Kasper

Rodilla, Verónica

Rodiño, Paula

Rodionov, Aleksandr

Rodionov, Ilya

Rodonò, Gianluca
Rodrigo, Peter John

Rodrigo-Clavero, María Elena

Rodrigues, Alexandre

Rodrigues, Alírio

Rodrigues, Andreia C. M.

Rodrigues, Antonio

Rodrigues, Cristina Fernanda Alves

Rodrigues, Eugénio

Rodrigues, Fernanda

Rodrigues, Frederico

Rodrigues, José Alberto

Rodrigues, José Inácio De Jesus

Rodrigues, Marco S.

Rodrigues, Maria Manuela Fernandes

Rodrigues, Mário S.

Rodrigues, Rafael V.

Rodrigues, Ricardo

Rodrigues, Sandra Sofia Quinteiro

Rodrigues-Fernandez, Alejandro

Rodríguez Amenedo, Jose Luis

Rodríguez De Rivera, Óscar

Rodriguez Diaz, Camilo Arturo

Rodríguez García, Antonio M

Rodríguez González, Álvaro

Rodríguez Millán, Marcos

Rodríguez Pasandín, Ana María

Rodríguez Prado, Daniel

Rodríguez Prieto, Gonzalo

Rodríguez Sanz, David

Rodríguez, Alfonso

Rodriguez, Alvaro

Rodriguez, Angel

Rodríguez, Camino Fernández

Rodriguez, Dionisio

Rodríguez, Javier

Rodríguez, José-Víctor

Rodríguez, Juan J.

Rodríguez, Manuel

Rodriguez, Nancy

Rodríguez, Orlando Camargo

Rodríguez, Oscar

Rodríguez, Ricardo Reyes

Rodriguez, Samuel

Rodriguez, Sergio

Rodríguez-Archilla, Alberto

Rodríguez-Avi, José

Rodríguez-Baena, Domingo Savio

Rodríguez-Cruz, M. Sonia

Rodriguez-Cuadrado, Sara

Rodríguez-Estévez, Vicente

Rodríguez-Gonzálvez, Pablo 
Rodriguez-Lozano, Francisco J. Rodríguez-Martínez, Alberto Rodríguez-Parada, Lucía Rodríguez-Peces, Martín Jesús Rodriguez-Pulido, Francisco J. Rodríguez-Rodríguez, Ignacio Rodríguez-Ruano, Sonia M. Rodríguez-Sanz, Álvaro Rodriguez-Vita, Juan

Rodziewicz, Pawel

Roedel, Christian

Roemer, Ulrich

Roga, Wojciech

Rogala, Andrzej

Rogala, Michał

Rogala, Zbigniew

Rogalski, Grzegorz

Roger, Daniel

Roggendorf, Hans

Roggendorf, Matthias J.

Rogié, Brice

Rogier, Francois

ROGKALA, AIKATERINI

Rogowski, Adam

Rogowski, Jan

Rogowski, Krzysztof

Rogozhnikov, Denis

Rogoziński, Tomasz

Roh, Chang-Gyun

Roh, Jaroslav

Roh, Jong Wook

Rohacs, Jozsef

Rohloff, Jens

Röhrig, Christof

Roj, Edward

Rój, Justyna

Rojas Sola, José

Rojas, Elisa

Rojas, Gabriel

Rojas-Cessa, Roberto

Rojek, Izabela

Rojí, Eduardo

Rojo, Teresa

Rojo-Álvarez, José Luis

Rokicki, Tomasz

Rokosz, Krzysztof

Roland, Theresa

Rolek, Jaroslaw

Rollenbeck, Rütger

Romagnoni, Piercarlo

Román Gallego, Jesús Ángel
Roman, Hector Eduardo

Roman, Kamil Krzysztof

Roman, Raul-Cristian

Romana, Manuel G.

Romanazzo, Sara

Román-Ibáñez, Vicente

Romano Spica, Vincenzo

Romano, Antonio

Romano, Diego

Romano, Donato

Romano, Luigi

Romano, Nicla

Romano, Silvia

Romano, Stefania

Romanowicz, Paweł J.

Romanowicz, Renata

Romanska-zapala, Anna

Románszki, Loránd

Rombaut, Evy

Romeira, Bruno

Romeo, Katerine

Romeo, Luca

Romeo, Saverio

Romero Collado, Angel

Romero Morales, Carlos

Romero Rodríguez, José María

Romero, Fernando Castano

Romero, Francisco J.

Romero, Francisco P.

Romero, Pedro

Romero-Ania, Alberto

Romero-García, Juan M.

Romero-González, Roberto

Romero-Sánchez, Francisco

Romić, Marija

Romijn, Constantijn

Rommel, Simon

Rompante Cunha, Carlos

Romuli, Sebastian

Ronca, Alfredo

Roncin, Kostia

Rondonuwu, Ferdy S.

Rong, Ruichen

Rong, Yu

Ronga, Luca Simone

Ronningen, Theodore J.

Ronsard, Larance

Roohi, Milad

Roohnikan, Mahdi

Root-Gutteridge, Holly

Roozen, Bernardus 
Ropelewska, Ewa

Roper, Courtney

Ropero, Jorge

Ropuszyńska-Surma, Edyta

Roque, Antonio

Rorrer, Greg

Ros, Frederic

Rosa, Alessandro

Rosa, Angelika Dorothea

Rosa, Bruno M.G.

Rosa, Flavio

Rosa, Pedro

Rosa, Rui Namorado

Rosaci, Domenico

Rosado Muñoz, Alfredo

Rosado, Jaime

Rosati Papini, Gastone Pietro

Rosati, Riccardo

Rosca, Sanda

Roscia, Mariacristina

Roscini, Francesca

Roscini, Luca

Roscow, James

Rose Marco, Delle

Rose, Georg

Roseiro, Luís

Roselli, Carlo

Roselli, Ivan

Rosen, Joseph

Rosenberg, Moshe

Rosenlund, Mats

Roseti, Livia

Roshanmanesh, Sanaz

Rosinová, Danica

Rosin-Paumier, Sandrine

Rosique Contreras, María Francisca

Rosker, Jernej

Roskos, Beverly

Rosłon, Jerzy

Rosner, Daniel

Ross, Brandon

Ross, Robert James

Rossano, Veronica

Rossato, Luca

Rossella, Francesco

Rossi, Claudio

Rossi, Daniele

Rossi, David

Rossi, Franca

Rossi, Giuliana

Rossi, Lorenzo
Rossi, Maddalena

Rossi, Massimiliano

Rossi, René Michel

Rossi, Stefano

Rossiter, Anthony

Rossiter, Sharon

Rössler, Peter

Rosso, Carlo

Rosso, Valeria

Rossomando, Francisco

Rostagno, Carlo

Rostami, Ali Bakhshandeh

Rostislav, Zemek

Roszak, Joanna

Roszak, Szczepan

Roszczenko-Jasinska, Paula

Roth, Andreas

Roth, Bradley J.

Roth, Johannes

Roth, Zvi

Rothbauer, Mario

Rothe, Sandra

Rothe, Stefan

Rothe, Sylvia

Rothkrantz, Leon

Roths, Johannes

Rotini, Federico

Rott, Eduard

Rottenstreich, Ori

Roua, Lajnaf

Roubík, Hynek

Roudsari, Sajjad Sayyar

Rouff, Christopher

Rouge, Pierre

Roumpos, Christos

Rousakis, Theodoros

Roussey, Catherine

Roussis, Ioannis G.

Routhu, Nanda Kishore

Roux, Jean François

Roux, Stéphane

Rovas, Alexandros

Roveda, Loris

Rowe, Brian

Rowell, Roger M.

Rowland, Clare

Roy Chowdhury, Sandipan

Roy, Anupam

Roy, Arnab

Roy, Dipankar

Roy, Partho Sarothi 
Roy, Sayan

Roy, Someshwar

Roy, Sumit

Rozbroj, Jiří

Różowicz, Antoni

Rozumek, Dariusz

Rozwadowski, Tomasz

Rozylo, Patryk

Różyło, Renata

Rozynek, Zbigniew

Rozzi, Elena

Ruan, Shanq-jang

Ruaro, Barbara

Rubegni, Pietro

Ruben, Pereira

Ruben, Rui B.

Rubiano, Jesús García

Rubin, Clinton T.

Rubin, Stuart

Rubini, Philip A.

Rubinić, Josip

Rubino, Felice

Rubino, Sandro

Rubinsztein-Dunlop, Halina

Rubio Alonso, Higinio

Rubio, Fernando

Rubio, Juan Luis

Rubio, Laura

Rubio, Ramón González

Rubio-Marcos, Fernando

Rübsamen, Nicole

Ruchkin, Ivan

Rucinska, Teresa

Rucki, Miroslaw

Rudakova, Natalya V.

Rudawska, Anna

Rudez, Urban

Rudnicki, Tomasz

Rudolf, Pavel

Rudolf, Rebeka

Rudzińska, Magdalena

Rudziński, Wojciech

Rudžionis, Žymantas

Ruelland, Eric

Ruffato, Gianluca

Ruffin, Paul

Ruffino, Barbara

Ruffino, Francesco

Ruggeri, Paolo

Ruggeri, Simona

Ruggieri, Sergio
Ruggiero, Alessandro

Rugnini, Lorenza

Rui, Zhenhua

Ruic, Dino

Ruipérez-Valiente, José A.

Ruivo, Celestino

Ruiz De Ballesteros, Odda

Ruiz Téllez, Trinidad

Ruiz, Magda

Ruiz, Maria Dolores

Ruiz, Vicente González

Ruiz-Altisent, Margarita

Ruiz-Caro, Roberto

Ruiz-Etcheverry, Laura

Ruiz-Femenia, Ruben

Ruiz-Lendínez, Juan José

Ruíz-Piñón, Manuel

Ruiz-Redondo, Aitor

Ruiz-Rosas, Ramiro Rafael

Ruiz-Zepeda, Francisco

Rukavishnikov, Victor

Rullmann, Michael

Rumora, Lada

Rumora, Luka

Rumpf, Clemens

Runco, Mark

Rundo, Leonardo

Runtuwene, Lucky Ronald

Rupakhety, Rajesh

Rupcic, Slavko

Rupeika-Apoga, Ramona

Rupenyan, Alisa

Ruppert, Kristine

Ruppert, László

Rurek, Mirosław

Rusca, Aura

Rusca, Florin Valentin

Rus-Casas, Catalina

Ruschin, Shlomo

Ruser, Heinrich

Rusi, Sergio

Rusinek, Alexis

Ruskin, Heather J.

Rusnak, Ilan

Rusowicz, Artur

Russ, John

Russak, Amos

Russo Krauss, Irene

Russo, Alessio

Russo, Antonia

Russo, Danilo 


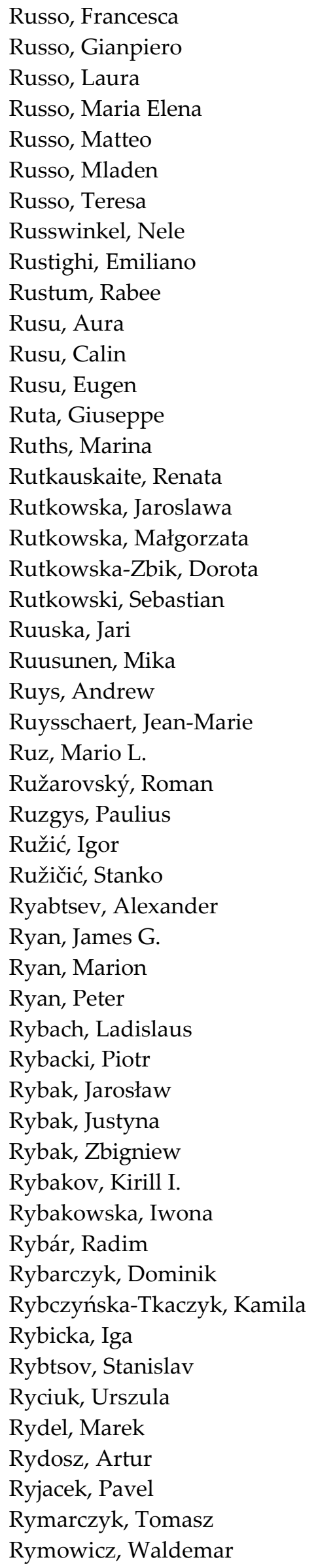

Ryms, Michal

Rymuza, Katarzyna

Ryndzionek, Roland

Rynkovskaya, Marina

Rynkowski, Piotr

Ryoo, Young-Jae

Ryou, Heonjune

Ryou, Hong Sun

Ryou, Jae-Suk

Rytel, Elżbieta

Ryu, Dong-Man

Ryu, Han-Youl

Ryu, Jae-Jun

Ryu, Jae-Myung

Ryu, Ji-won

Ryu, Jiyoon

Ryu, Jongkwan

Ryu, Junghee

Ryu, Junichi

Ryu, Keun Ho

Ryu, Kwang Sun

Ryu, Sangjin

Ryu, Seung Yoon

Ryvkin, Shmuel

Ryżak, Magdalena

Ryzhkov, Sergei

Rząsa, Krzysztof

Rześny-Cieplińska, Jagienka

Rzeszut, Katarzyna

Rzętała, Mariusz

Rzhanov, Yuri

Rzońca, Patryk

Rzucidlo, Pawel

Sá, Jacinto

Sá, Paulo

Sa'Ed, Jaser

Saadeh, Mohammad

Saafi, Mohamed

Saakes, Michel

Saatchi, Reza

Saati-Santamaría, Zaki

Sabaliauskas, Tomas

Šabanovič, Eldar

Sabater Navarro, Jose Maria

Sabater, Carlos

Sabatini, Marco

Sabatini, Roberto

Sabatini, Valentina

Sabbah, Mohammed

Sabban, Albert

Sabbatini Peverieri, Giuseppino 
Sabchevski, Svilen Petrov

Sabhahit, Jayalakshmi N.

Sabharwal, Arjun

Sabharwal, Chaman

Šabić Runjavec, Monika

Sabik, Agnieszka

Sabín, Carlos

Sabo, Cosmin

Sabol, Ivan

Sabounchi, Sepideh Seyedzadeh

Sabourin, Christophe

Sabzehgar, Reza

Sacala, Ioan

Sacchi, Giovanna

Sacco, Pier

Sachadyn-Król, Monika

Sachat, Alexandros El

Sachau, Delf

Sachithanandham, Jaiprasath

Sachkov, Victor

Sachlas, Athanasios

Sachmechi, Issac

Sachpazis, Costas

Sackl, Andreas

Sacomano Filho, Fernando Luiz

Saczuk, Klara

Sadar, Nadja

Sadeghi, Dr. Omidreza

Sadeghi, Farshid

Sadeghipour, Negar

Sadegh-Zadeh, Seyed-Ali

Sadik-Zada, Elkhan Richard

Sadiq, Ali Safaa

Sadjadi, Firooz

Sadowska, Anna

Sadowska, Kamila

Sadowska-Buraczewska, Barbara

Sadowski, Jarosław

Sadowski, Lukasz

Sadowski, Maciej

Sadre, Ramin

Sadykov, Vladislav

Šadzevičius, Raimondas

Saeed, Khalid

Saeed, Nagham

Saeed, Nasir

Saeedian, Meysam

Saeki, Kumiko

Saenz-Cogollo, Jose Francisco

Sæther, Stig Arve

Sáez Blázquez, Cristina
Safa, M Faisal

Safaei, Alireza

Safaei, Mohammad Reza

Safapour, Elnaz

Safar, Leos

Šafarič, Riko

Safdari Shadloo, Mostafa

Safeea, Mohammad

Saffache, Pascal

Saffari Pour, Mohsen

Saffari, Mohammad

Safi, Carl

Safonov, Alexander Aleksandrovich

Safronova, Tatiana

Sagiya, Takashi

Sagris, Dimitrios

Saha, Dipanjan

Saha, Dipongkor

Saha, Jhantu Kumar

Saha, Renata

Saha, Subhasish

Saha, Surya

Sahami, Amirreza

Saharan, Lalita

Sahin, Atakan

Sahmenko, Genadijs

Saho, Kenshi

Sahoo, Deepak

Sahoo, Subhransu

Sahu, Bharat J. R.

Sahu, Sushant

Sai, Hiroaki

Saija, Franz

Saijo, Yoshifumi

Saiko, Guennadi

Saikumar, Niranjan

Sain, Sunanda

Saini, Ikjot

Saini, Rajkumar

Saini, Ramesh Kumar

Sainidou, Rebecca

Saintenoy, Albane

Saint-Pol, Julien

Sainz De Abajo, Beatriz

Saito, Akira

Saito, Kozo

Saito, Yasuhiro

Saitou, Masatoshi

Saitou, Takashi

Saiz Jimenez, Cesareo

Sáiz Manzanares, María Consuelo 
Saiz, Fernan

Saiz-Fernández, Iñigo

Sajek, Adam

Sajid, Mohammad

Sajid, Muhammad

Sajjanhar, Atul

Sakač, Nikola

Sakaebe, Hikari

Sakagami, Kimihiro

Sakagami, Norimitsu

Sakai, Shigeki

Sakalauskas, Eligijus

Sakamoto, Maiko

Sakata, Shuzo

Sakhabutdinov, Airat Zh.

Sakharov, Alexey

Sakharova, Nataliya

Sakic, Pierre

Sakthivel, Kogularasu

Sala, Claudia

Sala, Gessica

Sałabun, Wojciech

Salach, Jacek

Salagean, Tudor

Saláková, Alena

Salam, Adul

Salameh, Najat

Salamí, Esther

Salami, Mohammad Reza

Salamin, Yannick

Salamone, Francesco

SALAPARE, Hernando III

Salas, José Blanco

Salawu, Abayomi

Salawu, Emmanuel

Salazar, Addisson

Sălceanu, Alexandru

Salchow-Hömmen, Christina

SALDAÑA, Miguel A. MARISCAL

Saldanha, Carlota

Saleh, Arash Emami

Saleh, Fatima A.

Salehfar, Hossein

Salehi, Amin

Salehi, Maryam

Salehi, Saeed

Salem, Ali

Salerno, Aurelio

Salerno-Kochan, Renata

Sales, Ester

Salgansky, Eugene Aleksandrovichn
Salgues, Marie

Salido Tercero, Jesús

Salim, Hani

Salim, Muath Bani

Salimon, Alexey

Salina, Gaetano

Salinas Rodríguez, Beatriz

Salinas, Carlota

Saller, Maximilian

Salman, Ahmad

Salman, Michael S.

Salnitri, Mattia

Salomó-Coll, Oscar

Salomon, Michel

Salonen, Heidi

Salonen, Laura

Salonitis, Konstantinos

Salonvaara, Mikael

Saltas, Vassilios

Saltas, Vassilis

Salud, Serrano

Salvador, Castillo-Rivera

Salvadori, Giacomo

Salvati, Daniele

Salvati, Enrico

Salvatori, Rosamaria

Salvatori, Stefano

Salvi, Massimo

Salvini, Antonella

Salvo, Andrea

Salvucci, Manuela

Salwin, Mariusz

Salzano, Ernesto

Salzano, Francesco

Samad, Yarjan Abdul

Samadi, Forooza

Samaitis, Vykintas

Samal, Sneha

Samanta, Soumitra

Samanta, Swagata

Samarakoon, Bhagya

Samaras, Ioannis

Samaras, Nicholas

Sambataro, Sergio

Samborski, Sylwester

Sambruno, Alejandro

Sambucci, Matteo

Sameer, Husam

Sametinger, Johannes

Samhan-Arias, Alejandro

Samiei, Salma 
Samil Demirkol, Ahmet

Sammartino, Gilberto

Samoilova, Olga

Sampaio, Pedro Nuno Sousa

Sampaolo, Angelo

Sampedro, Begoña Esther

Sams, Craig

Samsudin, Asep Muhamad

Samuel, Fawzy-Hosny

Samulionis, Vytautas

San Juan, Alejandro F.

San Roman, Julio

Sanabria Martin, Sergio José

Sanabria-Russo, Luis

Sanavia, Tiziana

Sánchez De Miguel, Alejandro

Sánchez Egea, Antonio J.

Sanchez Galan, Javier

Sánchez García-Vacas, Daniel

Sanchez- Gonzalez, Alberto

Sanchez Lasheras, Fernando

Sánchez López, Elena

Sanchez Ramos, Jose

Sánchez Rodríguez, Luis

Sánchez, Alberto

Sánchez, Antonio Bernardo

Sánchez, Borja Bordel

Sánchez, Isidro

Sanchez, Mauricio A.

Sanchez, Thomas

Sanchez, Victor

Sánchez-Alzola, Alberto

Sanchez-Cambronero, Santos

Sanchez-de-la-Muela, Alejandro-Miguel

Sanchez-Espiga, Javier

Sánchez-Fernández, José Ángel

Sánchez-García, David

Sanchez-Gomez, Martin

Sánchez-Gómez, Rubén

Sánchez-González, María Carmen

Sánchez-Hermosilla, Julián

Sánchez-Postigo, Alejandro

Sanchez-Rojas, Jose Luis

Sanchez-Romero, Jose-Luis

Sánchez-Salgado, Pablo

Sánchez-Soto, Luis L.

Sancho, Rafael

SANCIBRIAN, RAMON

Sandage, Mary J.

Sandberg, Marcus

Sandberg, Ulf
Sandfeld, Stefan

Sandoli, Antonio

Sandoval Arévalo, Juan Sebastián

Sandoval, Steven

Sands, David

Sands, Timothy

Sandsten, Maria

Sandu, Andrei Victor

Sandu, Ion

Sandu, Titus

Sandu, Viorel

Sandulescu, Robert

Sangwine, Stephen

Sangwongwanich, Ariya

Sanislav, Teodora

Saniuk, Anna

Saniuk, Sebastian

Sanjeet, Mehariya

Sanjurjo Sanchez, Jorge

Sankarasubramanian, Shrihari

Sankhaneel, Sinha

San-Miguel, Alfonso

Sannino, Diana

Sannino, Filomena

Sanphuang, Varittha

Sansonetti, Antonio

Sansoni, Paola

Sant, Paul

Santa, Fernando

Santagati, Cettina

Santamaría, Ignacio

Santamaria, José

Santamaria, Ulderico

Santarsiero, Giuseppe

Santhanam, Abirami

Santhiyagu, Vijay Joseph

Santi, Melissa

Santiago Chiquero, Isabel

Santiago, Molina

Santini, Antonello

Santini, Carlo

Santini, Silvia

Santisteban, Maria

Santoni, Andrea

Santoni, Francesco

Santonocito, Dario

Santoro, Francesco

Santoro, Mattia

Santos, Filipe Neves Dos

Santos, Gilberto

Santos, Henrique 
Santos, Jerran

Santos, Jesús Daniel

Santos, José Gomes Dos

Santos, José Maria

Santos, Luís Carlos

Santos, Marcos Vinicius

Santos, Mário João S. F.

Santos, Rafael M.

Santos, Ricardo

Santos, Rodrigo M.

Santos, Ruben

Santos, Telmo

Santos, Vitor

Santosa, Hendrik

Santos-Gago, Juan M.

Santos-García, Gustavo

Santucci, Valentino

Santulin, Marco

Santur, Yunus

Sañudo, Roberto

Sanwar Hosen, A. S. M.

Sanyal, Debankur

Sanz-Calcedo, Justo Garcia

Šapalas, Antanas

Sapana, Lohani

Sapienza, Vincenzo

Saponaro, Mirko

Saprykina, Yana

Sapsis, Themistoklis

Sapudom, Jiranuwat

Sara, Pensieri

Saracevic, Muzafer H.

Sarafraz, Mohammad Mohsen

Saraga, Dikaia E.

Saragadam, Vishwanath

Sarajlic, Mirza

Saraswati, Saraswati

Saratale, Ganesh Dattatraya

Saravanakumar, Kandasamy

Saravanan, Jothi

Sarcevic, Peter

Sarda, Edoardo

Sardari, Roya R. R.

Sardi, Alberto

Sardi, Giovanni Maria

Sardini, Emilio

Sardone, Rodolfo

Saremi, Mehdi

Sarfraz, Shoaib

Sargentis, George-Fivos

Sarhosis, Vasilis
Sarikov, Andrey

Sarkar, Abhijit

Sarkar, Biswajit

Sarkar, Sujoy

Sarkarai Nadar, Venkadesh

Sarker, Debalaya

Sarker, Iqbal H.

Sarlis, Nicholas

Sarniak, Mariusz

Sarnovsky, Martin

Saro, Cristina

Sarría, Francisco Alonso

Sarris, Ioannis E.

Sarry, Frédéric

Sarswat, Prashant K.

Sarti, Elena

Sarto, Francesca

Sartor, Francesco

Sartori, Maria

Sarzyńska, Justyna

Sas, Wojciech

Sasagawa, Kiyotaka

Sasai, Kazuto

Sasaki, Ken

Sasaki, Noriyuki

Sasaki, Yutaka

Saseendran, Vishnu

Sashiwa, Hitoshi

Sasiadek, Jurek Z.

Sasmito, Agus Pulung

Sassa, Shinji

Sassani, Alireza

Sassu, Mauro

Sastre, Mariano

Sastre-Santos, Angela

Satender, Kataria

Sathya, Vanlin

Šatínský, Dalibor

Satler, Massimo

Sato, Hayato

Sato, Hiroyuki

Sato, Kimiyasu

Sato, Manabu

Sato, Ryuta

Sato, Shingo

Sato, Shinobu

Sato, Shunsuke A.

Sato, Takuichi

Sátor, Ladislav

Satoshi, Iriyama

Satou, Ryouichi 
Satpute, Nitin

Sattar, Farook

Sattarov, Robert R.

Saucan, Emil

Saucedo Espinosa, Mario Alberto

Sauer, Hans Martin

Sauer, Nathalie

Sauer, Piotr

Saul, Dominik

Saura Lacárcel, José Ramón

Saurav, Kumar

Saurina, Javier

Sautot, Lucile

Sava, Alexandre

Savagatrup, Suchol

Savaglio, Claudio

Savaidis, Georgios

Savastano, Matteo

Savi, Filippo

Savill, Mark

Savin, Alexander

Savino, Giovanni

Savino, Sergio

Savio, Gianpaolo

Saviozzi, Matteo

Savkin, Andrey V.

Savoi, Stefania

Savu, Diana

Savuto, Elisa

Savvidou, Maria

Sawada, Kazuhiko

Sawada, Tadamasa

Sawada, Yoshihide

Sawicka, Barbara

Sawicki, Jerzy

Sawicki, Piotr

Sawicki, Rafal

Saxena, Prateek

Sayab, Mohammad

Sayarshad, Hamid R.

Saylam, Kutalmis

Sayood, Khalid

Sazonov, Igor

Sbarbati, Chiara

Sbarufatti, Claudio

Sberveglieri, Veronica

Sboev, Alexander

Scacco, Salvatore

Scaini, Denis

Scala, Barbara

Scala, Fabrizio
Scalari, Giacomo

Scalera, Lorenzo

Scalerandi, Marco

Scaman, Christine H.

Scamarcio, Alessandro

Scamoni, Fabio

Scandurra, Graziella

Scanlan, Aaron

Scappaticci, Lorenzo

Scaradozzi, David

Scaramozzino, Domenico

Scaramuzza, Matteo

Scarano, Antonio

Scardina, Giuseppe Alessandro

Scardulla, Francesco

Scaringi, Gianvito

Scarpa, Emanuele-Salvatore

Scarpelli, Giuseppe

Scarpete, Dan

Scarselli, Gennaro

Scarvell, Jennie

Scesi, Laura

Schaal, Wesley

Schabowicz, Krzysztof

Schaefer, Benjamin

Schäfer, André

Schäfer, Edgar

Schäfer, Helmut

Schaffarczyk, Peter

Schäffer, Beat

Schalch, Jacob

Scharoun Benson, Sara

Schaschke, Carl

Schauberger, Günther

Scheele, Fedde

Scheffler, Christiane

Scheidl, Rudolf

Schein, Jochen

Schelenz, Ralf

Schell, Juliana

Schenone, Silvia

Schepler, Kenneth

Scherberich, Arnaud

Scherer, Rafal

Scherillo, Fabio

Scherrer, Yves

Schiavi, Pier Giorgio

Schiavon, Marco

Schiavon, Michela

Schicchi, Diego Said

Schick, Evan 
Schieferstein, Eva

Schielen, Ralph M.J.

Schifano, Sebastiano Fabio

Schiffner, Martin

Schilberg, Daniel

Schiliro, Luca

Schilling, Frank-Peter

Schilling, Oleg

Schillinger, Burkhard

Schimmel, Saskia

Schimpe, Michael

Schiopu, Ionut

Schippa, Leonardo

Schiraldi, David

Schirone, Luigi

Schirru, Luca

SCHISSLER, ANDREW

Schito, Joram

Schito, Paolo

Schjønning, Per

Schlanbusch, Rune

Schlegel, Steffen

Schlenstedt, Christian

Schlesinger, Mark

Schlesinger, Martin

Schlippert, Dennis

Schlund, Sebastian

Schlüter, Nadine

Schmauss, Bernhard

Schmedake, Thomas A.

Schmeitz, Antoine

Schmelzer, Jürn W.P.

Schmid, Micaela

Schmidl, Doreen

Schmidt, Adam

Schmidt, Bernard

Schmidt, Franziska

Schmidt, Jan H.

Schmidt, Johanna

Schmidt, Olaf

Schmitt, Carlo

Schmitt, Jan

Schmitt, Katrin

Schmitt, Michael

Schmitz, Nico

Schmitz, Roland

Schmoch, Ulrich

Schnabel, Thomas

Schnabel, Uta

Schnaubelt, Sebastian

Schneckenburger, Herbert
Schneider, Marius

Schneider, Martin

Schneiderman, Emet

Schneider-Muntau, Barbara

Schnell, Robert

Schnuerer, Matthias

Schoepp-Cothenet, Barbara

Schoesser, Britta

Schöggl, Josef-Peter

Scholkmann, Felix

Scholl, Philipp M.

Schöllhorn, Wolfgang I.

Scholta, Joachim

Scholtz, Vladimír

Scholz, Ferdinand

Scholz, Johannes

Schönbauer, Bernd

Schonberg, William

Schönitzer, Veronika

Schöpfer, Jutta

Schöppner, Volker

Schorr, Mark S.

Schottroff, Felix

Schowalter, Marco

Schregle, Roland

Schreiber, Fabio A.

Schreiber, Juergen

Schrittesser, Bernd

Schröder, Agnes

Schröder, Christian

Schroeder, Grzegorz

Schroer, Martin

Schrötter, Martin

Schubert, Gerhard

Schuchardt, Mirjam

Schueller, Michael John

Schuhmann, Katharina

Schuldt, Frank

Schuldt, Steven

Schulien, Jennifer A.

Schulp, Jan A.

Schultz, Nick

Schultz, Paul

Schultze, Martin

Schultze, Steven R.

Schultze, Volkmar

Schulz, Alexander

Schulz, Benjamin L.

Schulz, Nicola

Schumacher, Brian

Schumacher, Udo 
Schüßler, Dominik

Schuster, Sebastian

Schuttrumpf, Holger

Schuurman, Michiel J.

Schwab, Hans Martin

Schwab, Paul

Schwaminger, Sebastian

Schwan, William

Schwandt, Carsten

Schwarz, Casey Minna

Schwarz, Chris

Schwarz, Dana

Schwarz, Jiří

Schwarz, Justus Arne

Schwarz, Michael

Schwarz, Stefan

Schwarzbach, Paul

Schwede, Dirk

Schwenker, Friedhelm

Schwenzel, Julian

Schwotzer, Matthias

Sciacchitano, Andrea

Sciacovelli, Adriano

Sciaini, Germán

Scialla, Stefania

Scianna, Andrea

Sciaraffa, Nicolina

Sciarretta, Francesca

Scibilia, Elena

Ścibisz, Iwona

Scimeca, Luca

Scimemi, Giuseppe Fileccia

Scioscia, Floriano

Sciuto, Gaetano Antonio

Sciuto, Grazia Lo

Scmidt Tome, Philip

Scoffield, Jessica

Scorpio, Michelangelo

Scorza, Andrea

Scotognella, Francesco

Scott, Daniel M.

Screpanti, Laura

Scrimin, Paolo

Scudiero, Olga

Scurtu, Ionut Cristian

Sdanghi, Giuseppe

Sdino, Leopoldo

Sdobnyakov, Nickolay Yu

Seabra, Filipa

Seaman, David

Sebakhy, Khaled O
Sebastian Marian, Zaharia

Sebastian, Rizal

Sebastian, Schlecht

Sebastiani, Diego

Sebben, Simone

Sebe, Francesc

Seca, Ana Maria Loureiro Da

Secciani, Nicola

Seceleanu, Cristina

Sechilariu, Manuela

Secu, Mihail

Sedláček, David

Sedlačík, Michal

Sedlák, Vladimír

Sedlar, Milan

Sedmidubský, David

Sedunov, Alexander

See, Chan

See, Tian Long

Seeboeck, Rita

Seedorf, Udo

Seeling, Patrick

Seferagić, Amina

Segall, Richard

Segarra, Mercé

Seghaye, Marie-Christine

Šegota, Suzana

Segovia, José J.

Segura Plaza, José Francisco

Segura, Ana

Segura, Juan-Jose

Segura, Rocio L.

Segura-Garcia, Jaume

Seh, Zhi Wei

Seiberl, Wolfgang

Seibt, Johanna

Seidel, David H.

Seidelmann, Ken

Seifan, Mostafa

Seifert, Gotthard

Seifi-Mofarah, Sajjad

Seijo, M. Carmen

Seiner, Brienne N.

Seip, Knut L

Seiphoori, Ali

Seitsonen, Oula

Seitz, Bjoern

Seitz, Rudolf

Sejkorová, Marie

Sejong, Oh

Sęk, Grzegorz 
Sek, Slawomir

Sekiguchi, Atsuko

Sekine, Masashi

SEKINE, Tomohito

Sekiya, Mizuki

Sekret, Robert

Sekulic, Damir

Selby-Pham, Sophie

Selci, Stefano

Seleiman, Mahmoud F.

Seleznev, Mikhail

Selezneva, Maria

Selim, Hossam

Seljan, Sanja

Sell, Nathan P.

Sellami, Akrem

Selleri, Stefano

Selles-Perez, Sergio

Sellitto, Andrea

Selopal, Gurpreet Singh

Selvan, Raghavendra

Selyanchyn, Roman

Semeniuta, Oleksandr

Semenov, Alexander

Semenov, Yuriy

Semisalova, Anna

Semjon, Boris

Semkov, Krum

Semlitsch, Bernhard

Sempiterno, Cristina

Semprini, Marianna

Sen Gupta, Sourav

Sen, Rwik

Sen, Sujat

Senadeera, Wijitha

Senalik, Christopher Adam

Senapathi, Deepa

Senatore, Rosa

Senchina, David

Senčić, Tomislav

Sene, Ndolane

Senesi, Giorgio

Senesi, Nicola

Senetakis, Konstantinos

Senetra, Adam

Senger, Moritz

SenGupta, Indranil

Senhadji-Navarro, Raouf

Şenilă, Marin

Senna, Carlos

Sentandreu, Miguel
Seo, Byung-Kuk

Seo, Dong-Woo

Seo, Hogeon

Seo, Hwajeong

Seo, Jihoon

Seo, Ji-Won

Seo, Joon Ho

Seo, Jung-Hee

Seo, Junho

Seo, Kazuya

Seo, Myung Won

Seo, Sang Hyun

Seo, Sang-Woo

SEO, Seog Chung

Seo, Youngguk

Seoane-Collazo, Patricia

Seok, Hyun

Seok, Junhee

Seong, Nam-Chul

Sepe, Raffaele

Sequeira, César A. C.

Sequeira, Claudia

Sequeira, João

Serackis, Arturas

Serafim, Andrada

Serajian, Reza

Serani, Andrea

ŞERBAN, Gheorghe

Șerban, Ovidiu

Seredin, Oleg

Seredyński, Mirosław

Seres, Jozsef

Šerevičienè, Vaida

Serfaty, Stéphane

Sergi, Pier Nicola

Sergio, Casas Yrurzum

Sergiou, Charalambos

Seri, Paolo

Seriani, Stefano

Serikov, Arkady

Seritan, George

Serjouei, Ahmad

Serra, Roger

Serra, Xavier

Serrancolí, Gil

Serrano, Daniel

Serrano, Javier Toledo

Serrano, Jose

Serrano, Luis

Serrano, Salvatore

Serrano-fontova, Alexandre 
Serrano-Montes, José Luis

Serrapica, Francesco

Serra-Ruiz, Jordi

Servi, Michaela

Sescu, Adrian

Sešek, Aleksander

Šešok, Dmitrij

Sessa, Salvatore

Sestakova, Janka

Sestras, Adriana F.

Sestras, Paul

Seteikin, Alexey

Setola, Roberto

Setoodeh Jahromy, Saman

Sett, Soumyadip

Setyaningsih, Widiastuti

Sever, Drago

Severino, Alessandro

Sevil, Hakki Erhan

Sevilla-Morán, Beatriz

Sewerniak, Piotr

Sewint, Nancy

Sextro, Walter

Seybold, Jonathan

Seyedaghazadeh, Banafsheh

Sezenna, Elena

Sfetsos, Athanasios

Sfondrini, Maria Francesca

Sforna, Marino

Sgambi, Luca

Sgarzani, Rossella

Sgherri, Cristina

Sgroi, Mauro Francesco

Sguazzo, Carmen

Shaaban, Ibrahim

Shabbir, Noman

Shadloo, Mostafa S.

Shafai, Cyrus

Shafer, Michael

Shafiee, Mahmood

Shafiei, Ali

Shafiq, Omair

Shafique, Muhammad

Shafiullah, GM

Shagar, Viknash

Shah, Farhed A.

Shah, Furqan A.

Shah, Jainil

Shah, Parag K

Shah, Rohan

Shah, Shagan
Shah, Syyed Adnan Raheel

Shah, Zahir

Shahab, Sina

Shahani, Uma

Shahbodagh, Babak

Shaheen, Mohamed A.

Shahian Jahromi, Babak

Shahmoradi, Javad

Shahnewaz, Md

Shahparasti, Mahdi

Shahpari, Morteza

Shahri, Abbas Abbaszadeh

Shahriar, Md Rifat

Shahrour, Isam

Shahverdiev, Elman

Shaik, Anver Basha

Shaik, Riyaaz Uddien

Shajahan, Sunoj

Shakibaei, Mehdi

Shakti, P. C.

Shakya, Akhilesh

Shala, Ahmet

Shalfawi, Shaher A. I.

Shamanaeva, Liudmila G.

Shamberger, Patrick

Shamir, Lior

Shamonin, Mikhail

Shams, S. Fatemeh

Shamseldin, Tamer

Shamshirband, Shahab

Shamshiri, Redmond R.

Shan, Hongming

Shan, Juan

Shan, Shengbo

Shan, Wenqian

Shan, Xin

Shan, Yongwei

Shanawani, Mazen

Shang, Junlong

Shang, Qingsen

Shang, Yilun

Shankar, Eswar

Shankles, Peter

Shanmugam, Muruganandan

Shanmugam, Shankar Ganapathi

Shannigrahi, Susmit

Shao, Dangdang

Shao, Huaiyu

Shao, Jianfu

Shao, Pei-Chi

Shao, Yaping 
Shaparev, Nikolay Y.

Sharaev, Maksim

Sharazi, Asif

Shariati, Ali

Sharif Razavian, Reza

Sharif, Amir

Sharifi, Mani

Sharipova, Altynai

Sharma, Abhisheak

Sharma, Abhishek

Sharma, Arvind

Sharma, Ashish

Sharma, Ashutosh

Sharma, Gaurav

Sharma, Gokarna

Sharma, Jivitesh

Sharma, Jyotsna

Sharma, Kshitij

Sharma, Lav

Sharma, Manik

Sharma, Manisha

Sharma, Pradip

Sharma, R. K.

Sharma, Suvash

Sharma, Vishal

Sharopov, Farukh

Sharvia, Septavera

Shashidhar, Narasimha

Shasthri, Sivaguru

Shaukat, Kamran

Shaula, Aliaksandr

Shavandi, Amin

Shavezipur, Mohammad K.

Shaw, Harry

Shaw, Sophie

Shaw-Cortez, Wenceslao

Shaygan, Mandana

Shbair, Wazen

Shchukina, Alexandra

She, Bing

Shearrer, Grace E.

Shehu, Rafael

Shekaramiz, Mohammad

Shekargoftar, Masoud

Shekhani, Husain

Shellaiah, Muthaiah

Shemelya, Corey

Shemesh, Moshe

Shen, Cheng

Shen, Chih-Hsiung

Shen, Eric
Shen, Jialong

Shen, Kai

Shen, Ke

Shen, Ruiqing

Shen, Shanpu

Shen, Shuilong

Shen, Wen Zhong

Shen, $X \mathrm{i}$

Shen, Xiangying

Shen, $Y i$

Shen, Yuecheng

Shen, Zhenyu

Shen, Zhiqi

Shenashen, Mohamed A.

Sheng, Jia

Sheng, Jianxiong

Sheng, Jun

Sheng, Ruilong

Shentsov, Volodymyr

Shepherd, Lynsay A.

Sher, Farooq

Sherbakov, Sergei

Sherry, Jesse

Sherry, Lance

Shertzer, Howard G.

Sherwood, James

Sheshadri, Karthik

Shestopalov, Alexander M.

Sheu, Guang Yih

Sheu, Ruey-Kai

Shevate, Rahul

Shevchenko, Sergey

Shevchuk, Igor

Shevkunov, Igor

Shevtsov, Sergey

Shewarega, Fekadu

Sheyn, Dmitriy

Sheynin, Yuriy

Shi, Chengzhi

Shi, Jie

Shi, Jingyi

Shi, Jingyu

Shi, Junhui

Shi, Junwei

Shi, Lishian

Shi, Qiongfeng

Shi, Rongye

Shi, Xijun

Shi, Xiyu

Shi, Yong

Shi, Yu 
Shiau, Jiun-Yan

Shibaev, Petr

Shibata, Naoki

Shieh, Chin-Shiuh

Shieh, Hsin-Jang

Shieh, Wann-Yun

Shih, Ching-long

Shih, Chun-Kuang

Shih, Dong-Her

Shih, Tianyuan

Shih, Tom

Shikhantsov, Sergei

Shiku, Hitoshi

Shilev, Stefan

Shilov, Nikolay

Shim, Changsu

Shim, Jae-Kyung

Shimada, Kunio

Shimano, Kenjiro

Shimazu, Haruki

Shimizu, Kazuo

Shimizu, Yuki

Shimohata, Takaaki

Shimomura, Takumi

Shimomura, Yoshiki

Shin, Crystal S.

Shin, Donghee

Shin, Jeong-Heon

Shin, Jitae

Shin, Jong-Ho

Shin, KwangSup

Shin, Minchul

Shin, Soo Young

Shin, Tea Ho

Shin, Woosuk

Shin, Yong Jae

Shin, Youngjae

Shin, Yun-ho

Shinada, Tetsuro

Shinmura, Fumito

Shinoara, Naoki

Shinohara, Russell

Shinya, Akikazu

Shioda, Shigeo

Shiomori, Koichiro

Shirahata, Katsushi

Shiratani, Masaharu

Shirazinejad, Reza

Shirinskaya, Anna

Shiroma, Wayne A.

Shirvani, Zeinab
Shirvanimoghaddam, Kamyar

Shiryayev, Oleg

Shishatskiy, Sergey

Shishehbor, Mehdi

Shishkin, Andrei

Shiu, Eric C.

Shiu, Hung-Jr

Shiu, Yi-Shiang

Shivakumar, Vibhav Bharadwaj

Shivarudrappa, Shivakumar K.

Shiwakoti, Santosh

Shiyomi, Masae

Shmatko, Natalia

Shnorhokian, Shahe

Shoaib, Sultan

Shoda, Ayaka

Shoda, Makoto

Shoeb, Asaduzzaman

Shojaei, Arman

Shojaeighadikolaei, Amin

Shojafar, Mohammad

Shokouhimehr, Mohammadreza

Shokrolah Shirazi, Mohammad

Shokrollahi, Peyman

Shomvanshi, Shivangi

Shon, Taeshik

Shooshtarian, Salman

Shoshany, Maxim

Shotton, Elizabeth

Show, Pau Loke

Shoydin, Sergey

Shrestha, Anish Prasad

Shrestha, Namita

Shrestha, Nisha

Shrestha, Shikha

Shrestha, Shweta

Shrestha, Sudhir

Shu, Yanfeng

Shugurov, Artur R.

Shukla, Anand Prakash

Shukla, Pratik P.

Shukla, Shivakant

Shults, Roman

Shum, Henry

Shum, Kenneth

Shunmugasamy, Vasanth Chakravarthy

Shvetsov, Evgeny

Shvetsova, Olga A.

Shvidchenko, Aleksandr V.

Shyu, Shiuh-Hwa

Siami-Namin, Akbar 
Šiaučiūnas, Raimundas

Sibilio, Sergio

Sibilla, Maurizio

Sibilski, Krzysztof

Šic Žlabur, Jana

Sicard, Pierre

Siciliano, Antonietta

Sicking, Dean L.

Siczek, Krzysztof

Siddiq, M. Amir

Siddique, Abu Bakar

Siddiqui, Rehan

Siddiqui, Salman I.

Siderska, Julia

Sidorenko, Anatolie

Sidorov, Denis Nikolai

Sidorov, Nikolay V.

Siebert, J. Paul

Siebert, Julien

Sieciński, Szymon

Siedliska, Anna

Siegert, Ingo

Sielużycki, Cezary

Siemianowski, Oskar

Siemiatkowska, Barbara

Siemion, Agnieszka

Sieni, Elisabetta

Sienkiewicz, Łukasz

Sier, Cornelis

Sierra, Jesus Enrique

Sierra-Castaner, Manuel

Sierra-Fernandez, A.

Sierra-Sosa, Daniel

Siew, Renard

Sifa, Rafet

Sifakakis, Iosif

Šiffalović, Peter

Siger, Aleksander

Sigmund, Zvonko

Signore, Giovanni

Signorini, Cesare

Signoroni, Alberto

Sik Jeon, Euy

Sikdar, Shirsendu

Sikder, Sujit

Sik-Lanyi, Cecilia

Sik-Lányi, Cecília

Sikora, Andrzej

Sikora, Elżbieta

Sikora, Janusz

Sikora, Malgorzata
Sikora, Marjan

Sikora, Paweł

Sikora, Roman

Sikora-Fernandez, Dorota

Sikorska, Anna

Sikorski, Wojciech

Sikström, Fredrik

Silaghi, Marius

Silea, Ioan

Šilha, David

Silhavy, Petr

Silhavy, Radek

Silic, Marin

Šiljak, Harun

Siller, Hector

Sillero, Amalia

Sillup, George P.

Silva Llanca, Luis

Silva, Adão

Silva, Ana Luisa

Silva, André R R

Silva, António

Silva, Arlindo

Silva, Catarina

Silva, Cristiana

Silva, Cristiano M.

Silva, Eliana Costa E

Silva, Lígia Torres

Silva, Margarida F. B.

Silva, Natascha Platz Batista Da

Silva, Nuno Helder Da Cruz Simões

Silva, Paula F.

Silva, Tiago

Silva, Walter A.

Silva-Perez, Manuel

Silveira, Célia

Silverio, Vania

Silvestre, Javier

Silvestre, Miguel

Silvestre, Santiago

Silvestri, Marco

Silvestri, Stefano

Silvia, Lorenzo Freire

Silwal, Baikuntha

Sim, Edith

Sim, Sang Jun

Sima, Vasile

Simakov, Sergey

Simanaviciene, Zaneta

Simani, Silvio

Simanjuntak, Firman Mangasa 
Simari, Cataldo

Simate, Isaac N.

Šimek, Jiř́i

Šimek, Milan

Simeonov, Vasil

Simic, Marko

Simion, Georgiana

Simitzi, Chara

Simm, Stefan

Simmini, Francesco

Simmonds, Paul J.

Simoes, Pedro

Simon, Balint

Simon, Barbara

Simon, Christian

Simon, David

Simon, Márta

Simon, Vilmos

Simon-Chane, Camille

Simone, Gabriele

Simonescu, Claudia Maria

Simoni, Francesco

Simón-Martínez, Cristina

Simonot, Lionel

Simonov, Nikolai

Simo-Tagne, Merlin

Simou, Stavros

Simpson, Jeffrey D.

Simsek, Murat

Simsek, Serhat

Šimšík, Dušan

Simson, Alan

Simurda, Tomas

Sinanan, Jolynna

Sinatra, Rosario

Sinay, Juraj

Sinclair, Anthony N.

Sinclair, Wade $\mathrm{H}$.

Sinex, Donal G.

Sing, Swee Leong

Singaraju, Aditya

Singaravelu, Ragunath

Singh, Aditi

Singh, Ajay Vikram

Singh, Amardeep

Singh, Bhupinder

Singh, Gurwinder

Singh, Harsh V. P.

Singh, Jai

Singh, Kamal Deep

Singh, Keshav
Singh, Kulbir

Singh, Raghuveer

Singh, Rahul Kumar

Singh, Rajani

Singh, Rajendra

Singh, Rajesh

Singh, Rajneesh

Singh, Ranjan

Singh, Ratnakar

Singh, Rituraj

Singh, Saurabh

Singh, Shakti

Singh, Surya P.

Singh, Uday

SINGH, Utkarsh

Singh, Veena

Singh, Vishal

Singh, Yogang

Singha, Subhankar

Singovszka, Eva

Sinha, Rashmi Sharan

Sinha, Sudarson

Sinha, Sudarson Sekhar

Sinha, Sunil

Sinharoy, Arindam

Sinibaldi, Edoardo

Sinicropi, Adalgisa

Sinicropi, Maria

Siniscalchi, Sabato Marco

Sinjab, Khaled

Sinjari, Bruna

Sinkiewicz-Darol, Elena

Sinnott, Michael L.

Sinou, Jean-Jacques

Siodla, Krzysztof

Sioma, Andrzej

Siomau, Michael

Siontis, George C. M.

Sioshansi, Ramteen

Sipos, Emilia

Siramshetty, Vishal B.

Sirbu, Daniela

Siregar, Syahril

Sirimulla, Suman

Sirkeli, Vadim P.

Sirmacek, Beril

Sirutkaitis, Valdas

Siska, Filip

Sisto, Renata

Sisworahardjo, Nur

Sitányiová, Dana 
Sitaraman, Hariswaran

Sitarek, Przemysław

Sitar-Tăut, Dan-Andrei

Sitek, Paweł

Sitnik, Lech J.

Siu, King Man

Siudem, Paweł

Sivaev, Igor B.

Sivaguru, Mayandi

Sivák, Peter

Sivakumar, Poopalasingam

Sivarajan, Saravanan

Sivasubramanian, Jayahar

Sivathanu, Vivek

Sivayogan, Gajarajan

Siverns, James

Siwicki, Piotr

Siwiec, Tadeusz

Siwik, Leszek

Siwińska, Agata

Siwiński, Jarosław

Skaf, Marta

Skaggs, Sheldon

Skalniak, Lukasz

Skalomenos, Konstantinos

Skarlatos, Dimitrios

Skawina, Bartlomiej

Skelin, Ana Kuzmanić

Skender, Ana

Skendrović

Skibicki, Jacek

Skilodimou, Hariklia D.

Sklorz, Martin

Skoczeń, Andrzej

Skoczkowski, Tadeusz

Skoczylas, Agnieszka

Skoczylas, Łukasz

Skodras, Athanassios

Skolud, Bozena

Skouras, Athanasios

Skowron, Krzysztof

Skowroński, Rafal

Skrickij, Viktor

Škrinjar, Jasmina Pašagić

Skřínský, Jan

Škrlj, Blaž

Skruch, Paweł

Skruzny, Michal

Skrylnyk, Oleksandr

Skrzypacz, Janusz

Skrzypczyński, Piotr
Skrzypkowski, Krzysztof

Skublov, Sergey G.

Skurikhin, Evgenii Germanovich

Skutnik, Zdzislaw

Słaby, Ewa

Sładek, Jerzy A.

Sładkowski, Aleksander

Sładkowski, Jan

Śladowski, Grzegorz

Slaets, Peter

Slámová, Martina

Slanina, Zdenek

Slaný, Michal

Slaski, Grzegorz

Slavič, Janko

Slavin, Rocky

Slavinskis, Andris

Slavov, Stoyan

Slawinski, Cezary

Śledziewski, Krzysztof

Ślefarski, Rafał

Slemr, Franz

Slesarenko, Viacheslav

Ślęzak, Marta

Ślęzak, Radosław

Ślęzak, Tomasz

Sliwa, Tomasz

Śliwiński, Jacek

Śliwiński, Paweł

Śliwka, Małgorzata

Sližytè, Danutè

Šljivo, Amina

Słoczyńska, Karolina

Slokar Benić, Ljerka

Slominski, Andrzej

Słomkiewicz, Piotr Marek

Sloop, Joseph

Ślosarczyk, Agnieszka

Sługocki, Łukasz

Ślusarczyk, Beata

Ślusarczyk, Łukasz

Ślusarczyk, Sylwester

Slyne, Frank

Smaga, Łukasz

Smaldone, Giorgio

Smalec, Agnieszka

Smani, Younes

Smardzewski, Jerzy

Smart, Lindsey

Smarzewski, Piotr

Smekal, Zdenek 
Śmieja, Marek

Smiesko, Martin

Śmiglak-Krajewska, Magdalena

Smirnov, Ivan V.

Smirnov, Nickolay N.

Smirnov, Vladimir M.

Smirnovs, Juris

Smith, Alex

Smith, Carol

Smith, Graham T.

Smith, Henry I.

Smith, James

Smith, Joseph D.

Smith, Kerry

Smith, Leonard B.

Smith, Mark

Smith, Michael C.

Smith, Robert

Smith, Zacharias J.

Smolacg, Klaudia

Smoleń, Andrzej

Smoliner, Juergen

Smołka, Jakub

Smondrk, Maros

Smreczak, Bozena

Smrž, Martin

Smuleac, Laura

Smułek, Wojciech

Smulko, Janusz

Smutka, Luboš

Smutny, Vladimir

Smyk, Bogdan

Smyk, Emil

Snow, Nicholas H.

Snowsill, Tristan

So, Byoungjin

So, Kwok Kan

So, Wi-Young

Soare, Andreea

Soares Júnior, Amílcar

Soares, Cristina

Soares, Denise Paschoal

Soares, Joao

Soares, Vasco N. G. J.

Sobaszek, Łukasz

Sobaszek, Michal

Sobczak, Paweł

Sobczyk, Bartosz

Sobczyk, Tadeusz

Sobecki, Janusz

Sobeslav, Vladimir
Sobianowska-Turek, Agnieszka

Sobieraj-Żłobińska, Anna

Söbke, Heinrich

Soból, Emil

Sobola, Dinara

Sobolev, Dmitri

Sobolewska, Olga

Sobolewski, Peter

Sobota, Aleksander

Sobotka, Piotr

Sobotova, Lydia

Šobra, Jan

Sobral, José Augusto Da Silva

Sobrido-Cameán, Daniel

Sobus, Jan

Socas, Rafael

Soccalingame, Lata

Soco, Eleonora

Socol, Marcela

Socoró Carrié, Joan Claudi

Soda, Josko

Sodano, Federica

Sodhro, Ali Hassan

Sodnik, Jaka

soeta, Yoshiharu

Sofia, Daniele

Sogorb Sánchez, Miguel Ángel

Söhling, Nicolas

Sohn, Insoo

Sohn, Jung Woo

Sohn, SungWoo

Sohrabi, Reza

Sohrabi, Salman

Șoimoșan, Teodora Melania

Sójka, Lukasz

Sojka, Mariusz

Sokolov, Oleg

Sokolovskij, Edgar

Sokołowski, Andrzej

Sol, Joeri

Sola, Daniel

Solachidis, Vassilios

Sola-Guirado, Rafael-Ruben

Solaimanian, Mansour

Solak, Krzysztof

Solanas, Agusti

Solanes, J. Ernesto

Soldatkina, Liudmyla

Soleimani, Majid

Soleimani-Mohseni, Mohsen

Soler, Jordi 
Solina, Vittorio

Solís-Lemus, José Alonso

Solitro, Giovanni Francesco

Sollmann, Nico

Soloducho, Jadwiga

Solomey, Nickolas

Solomon, Barry

Solomon, Lee

Solomon, Melani

Solomon, Nancy

Solonin, Alexey

Soloshonok, Vadim A.

Soloviev, Alexander

Solovieva, Anastasiya

Solsona, Francesc

Soltani, Madjid

Soltani, Mehdi

Soltaninejad, Mohammadreza

Solteiro Pires, Eduardo José

Soltysik, Bartłomiej

Sołtysik, Maciej

Sołtysik-Piorunkiewicz, Anna

Solyanikova, Inna

Soma, Gian Giuseppe

Soman, Rohan

Somma, Renato

Somnath, Suhas

Šomplák, Radovan

Son, Jongsang

Son, Jung-Young

Son, Min

Son, Su-Won

Son, Tae Yun

Son, Yeongkwon

Son, Youngdoo

Sonavane, Sheetal

Sonenberg, Liz

Sones, Amerian D.

Song, Aiguo

Song, Bomi

Song, Erdong

Song, Guoqing

Song, Hanjung

Song, Hengxu

Song, Homin

Song, Houbing

Song, Ickhyun

Song, Jian

Song, Jong-Won

Song, Jun

Song, Junesol
Song, Jungsuk

Song, Kenan

Song, Nam Woong

Song, Qinghua

Song, Tai-Jin

Song, Tai-Kyong

Song, Wen

Song, Won-Kyung

Song, Yang

Song, Yang Ho

Song, Yongze

Song, Yuhe Tony

Songtao, Liu

Sonnen, Andreas

Sonnino, Giorgio

Sonowal, Himangshu

Sonsteby, Henrik Hovde

Sontacchi, Alois

Sonwalkar, Nishikant (Nish)

Soomro, Mahfooz

Soomro, Mumtaz Hussain

Sorandaru, Ciprian

Sorbello, Gino

Sorbye, Sveinung Wergeland

Sorcar, Saurav

Sorescu, Ana Alexandra

Soriano, Jose A.

Soriano, José Miguel

Soriano, Julio Gómez

Soriano, Prof. Enrique

Soriano, Thierry

Sorli, Brice

Sorli, Massimo

Sornay-Rendu, Elisabeth

Sornek, Krzysztof

Sorrenti, Matteo

Sorrentino, Alessandra

Sorrentino, Andrea

Sorrosal-Forradellas, Maria Teresa

Sortino, Marco

Sørup, Hjalte Jomo Danielsen

Sorzano, Carlos Oscar Sanchez

Sosa Díaz, Teresa

Sosa González, Carlos Javier

Sosnina, Elena

Sosnowski, Janusz

Sosnowski, Marcin

Sotelo Monge, Marco Antonio

Sotillo, Belén

Sotiriadis, Konstantinos

Sotiriadis, Paul 
Sotiroudis, Sotirios P.

Soto-Varela, Roberto

Souayfane, Farah

Soufan, Othman

Soukal, Frantisek

Soulika, Athena

Souravlas, Stavros

Sousa Vieira, María Estrella

Sousa, Ana I.

Sousa, Angela

Sousa, António

Sousa, Bruno Miguel

Sousa, Cristina

Sousa, Daniel

Sousa, Hélder S.

Sousa, Joana L. C.

Sousa, Joao Miguel

Sousa, Joaquim J.

Sousa, Luís

Sousa, Xulio

Sousan, Sinan

Sousa-Silva, Rui

Soust-Verdaguer, Bernardette

Souto, André

Souza, Emerson

Sova, Daniela

Sova, Matej

Sovak, Guy

Sovilla, Betty

Sowale, Ayodeji

Sowden, Miles

Sówka, Izabela

Spacher, Peter

Spada, Lorenzo

Spagnoli, Andrea

Spagnolo, Paolo

Spagnolo, Vincenzo

Spagnuolo, Gianrico

Spagnuolo, Giovanni

Spall, James C.

Spallone, Roberta

Španić, Nikola

Špánik, Ivan

Spanka, Marina

Spano, Giuseppe

Spano, Lucio Davide

Spano, Tyler L.

Spanopoulos, Ioannis

Spanoudakis, Polychronis

Sparavigna, Amelia Carolina

Specht, Mariusz
Speliotis, Thanassis

Spencer, Adrian

Spencer, Lissa

Spendier, Kathrin

Speranza, Domenico

Speranza, Giorgio

Speranza, Vito

Speranzini, Emanuela

Sperber, GH

Sperli, Giancarlo

Spiehl, Dieter

Spiehs, Mindy J.

Spier, Carlos

Spieß, Lothar

Spigno, Giorgia

Špiláček, Michal

Spiliotis, Evangelos

Spiliotopoulos, Dimitris

Spinas, Enrico

Spinato, Sergio

Spinazzè, Andrea

Spinelle, Laurent

Spinelli, Antonello E.

Spinello, Davide

Spinsante, Susanna

Spiridigliozzi, Luca

Spitzenstetter, Florence

Spivak, Yu M.

Spizzichino, Valeria

Splichal, Igor

Spoerri, Loredana

Spoladore, Daniele

Spolaor, Fabiola

Spotorno, Roberto

Spowage, Andrew

Sprague, Michael A.

Spratford, Wayne

Sprave, Tanja

Sprynskyy, Myroslav

Spyridis, Panagiotis

Spyridonos, Panagiota

Spyrou, Evaggelos

Spyrou, Kostas J.

Squeglia, Nunziante

Squeo, Giacomo

Sredenšek, Klemen

Średnicka-Tober, Dominika

Sreedharan, Sreejesh

Sreeram, Anand

Srinivasan, Sabarathinam

Srinivasan, Seshadhri 
Srinivasan, Sowmya

Sriperumbudur, Kiran K.

Sriram, Lalitha Madhavi Konila

Sriskandaraja, Kaavya

Srivastava, Akriti

Srivastava, Deepchandra

Srivastava, Gautam

Srivastava, Hari Mohan

Srivastava, Madhur

Srivastava, Mugdha

Srivastava, Rekha

Środa, Marcin

Sroka, Ryszard

Sroka, Zbigniew J.

Sroka-Bartnicka, Anna

Srokosz, Piotr

Srotin, Igor S.

Sry, Vannei

Srzic, Veljko

Stabauer, Martin

Stacchi, Claudio

Stachewicz, Urszula

Stachurski, Wojciech

Stacul, Stefano

Staderini, Edoardo

Stahl, Karsten

Ståhle, Per

Staicu, Angela

Štajduhar, Andrija

Štajduhar, Ivan

Stal, Cornelis

Stam, Rianne

Stamate, Eugen

Stamatelatou, Katerina

Stamatescu, Grigore

Stamatiou, Yannis C.

Stamatopoulos, Constantine A.

Stamou, Adamantia

Stan, Claudiu

Stanaszek-Tomal, Elżbieta

Stancalie, Gheorghe

Stancampiano, Augusto

Stančiaková, Lucia

Stanciu, Gabriela-Dumitrita

Stanciu, Loredana

Stanciu, Mariana Domnica

Stanculescu, Marilena

Stańczyk, Justyna

Standish, Russell

Stanek-Tarkowska, Jadwiga

Stanescu, Ioana Andreea
Stanescu, Michaela Dina

Stănescu, Nicolae-Doru

Stanev, Valentin

Staniak, Mariola

Stanish, Lee

Stanišić, Svetlana

Staniszewski, Michał

Stanivuk, Tatjana

Stankevich, Nataliya

Stanko, Davor

Stanković, Siniša

Stanley, John

Stanula, Arkadiusz

Stapelmann, Katharina

Starčević, Damir

Staretu, Ionel

Štarha, Pavel

Starman, Bojan

Starnoni, Michele

Starościak, Wojciech

Starosta, Robert

Starsich, Fabian

Starykevich, Maksim

Stasa, Pavel

Stasiak-Różańska, Lidia

Stasiolek, Mariusz

Stassinakis, Argyris N.

Št’astník, Ondřej

Stastny, Petr

Staszak, Zaneta

Staszek, Kamil

Staszuk, Marcin

Stateczny, Andrzej

Staubli, Thomas

Stavi, Ilan

Stavrakakis, George S.

Stavrou, Vasilis

Stavroulakis, Georgios E.

Stawarz, Marcin

Stebbings, Georgina

Stebel, Kerstin

Stecklina, Oliver

Stecko, Justyna

Steenaard, Rebecca V.

Stefan, Markiewicz Jakub

Stefan, Simona Catalina

Stefanakis, Alexandros I.

Stefanakis, Nikolaos

Stefańczyk, Maciej

Stefanelli, Dario

Stefanelli, Manuela 
Stefanescu, Lucrina

Stefani, Oliver

Stefanidis, Kostas

Stefanini, Cesare

Stefaniuk, Dawid

Stefanizzi, Michele

Stefanizzi, Pietro

Stefano, Tornincasa

Stefanou, George

Stefanov, Krassen

Stefanowicz-Hajduk, Justyna

Steffi, Chris

Stefu, Nicoleta

Stegall, Paul

Stegenta-Dąbrowska, Sylwia

Steinbach, David

Steinbacher, Matthias

Steinbach-Rankins, Jill

Steinberg, Ely

Steinberg, Spencer

Steingartner, William

Steininger, Andreas

Steinmann, Stephan N.

Steinmann, Zoran

Steiros, Konstantinos

Stellato, Francesco

Steller, Janusz

Stelmashuk, Vitaliy

Stelson, Kim A.

Stelzer, Franz

Stencel, Marek

Stendahl, Olle

Stender, Merten

Stenstrøm, Yngve

Stepanov, Gennady V.

Stepenko, Serhii

Stephanedes, Yorgos

Stephen, Michael Rajesh

Stephen, Neil G.

Stepien, Anna

Stępień, Grzegorz

Stepien, Jacek

Stepien, Krzysztof

Stępniak, Katarzyna

Stępniowski, Wojciech

Sterba, Johannes H.

Sterenborg, Henricus Jcm C. M.

Sterner, Olov

Sterzyński, Tomasz

Stesina, Fabrizio

Stetter, Ralf
Stevanovic, Ivica

Stevens, Francis

Stevens, Michael

Stevens, Robin

Stevulova, Nadezda

Steward, Robert

Stewart, George C.

Stewart, Rebecca

Stewart, Todd

Sthal, Fabrice

Stievano, Igor

Štigler, Jaroslav

Stiglic, Gregor

Stine, Keith J.

Stipanicev, Darko

Stirling, Rod

Stiros, Stathis C.

Stjepan, Spalj

Stocchero, Matteo

Stoch, Paweł

Stochino, Flavio

Stöcker, Fabian

Stockill, Robert

Stoddard, Shana

Stodola, Petr

Stoeck, Tomasz

Stoffregen, Thomas

Stoia, Dan Ioan

Stoian, Vlad

Stoicuţă, Olimpiu

Stoilova, Svetla

Stojanoff, Vivian

Stojanovic, Branka

Stojkov, Marinko

Stojmenova, Kristina

Stoklas, Roman

Stolz, Ronny

Stoppini, Aurelio

Stormont, John

Stornaiuolo, Mariano

Stornelli, Vincenzo

Storsberg, Joachim

Storti, Enrico

Stortini, Angela

Story, Brett

Stouffs, Rudi

Støvring, Jan Luxhøj

Stoyanov, Borislav

Strąkowska, Anna

Strambini, Lucanos

Stranghöner, Natalie 
Strangwood, Martin

Strankowska, Justyna

Strappe, Padraig

Štraub, Daniel

Strauss, Alfred

Strazdins, Peter

Straže, Aleš

Streckbein, Philipp

Streckiene, Giedre

Strehmel, Veronika

Streitferdt, Detlef

Stręk, Tomasz

Strelec, Stjepan

Strelniker, Yakov

Strielkowski, Wadim

Strîmbei, Cătălin

Stringam, Betsy

Stringam, Blair L.

Strle, Drago

Strnad, Damjan

Strnad, Radek

Strobl, Jan

Strobl, Josef

Stroe, Daniel-Ioan

Stroh, Alexander

Strohbach, Anne

Strohmaier, Walter L.

Strohmayer, Andreas

Strozzi, Antonio

Strozzi, Matteo

Strube, Jochen

Strug, Barbara

STRUKOVÁ, Zuzana

Strumillo, Pawel

Strungaru, Stefan-Adrian

Strupler, Michael

Struzik, Artur

Struzik, Michal

Strzałka, Dominik

Strzecha, Krzysztof

Strzelecki, Artur

Strzelecki, Michal

Strzelecki, Przemysław

Stubbs, Christopher J.

Štubňa, Igor

Stucchi, Marta

Stuckey, David C.

Studebaker, Glenn E.

Studzinski, Jan

Studziński, Robert

Stuerga, Didier
Štular, Benjamin

Stull, Jamie A.

Stulrajter, Marek

Štumberger, Gorazd

Stuparu, Adrian

Sturari, Mirco

Sturm, Arnon

Sturm, Bob L.

Stuzhin, Pavel

Štverková, Hana

St-Yves, Ghislain

$\mathrm{Su}, \mathrm{Bo}-\mathrm{chiuan}$

$\mathrm{Su}$, Chung-Hwei

$\mathrm{Su}$, Chunhua

Su, Hang

$\mathrm{Su}$, Junwei

$\mathrm{Su}$, Kuo-Chih

$\mathrm{Su}$, Ming-Shou

$\mathrm{Su}$, Pi-Guey

$\mathrm{Su}$, Tsung-chow

$\mathrm{Su}$, Tung-Ching

$\mathrm{Su}$, Wei-Jiun

$\mathrm{Su}, \mathrm{Wu}-\mathrm{Chung}$

$\mathrm{Su}$, Yuan-Fong

$\mathrm{Su}$, Zhoucheng

Suárez, Alberto Jiménez

Suarez, Alejandro

Suarez, Alvaro

Suárez, Eduardo

Suarez, Elisabet

Suárez, Fernando

Suarez, Maria J.

Suárez-Carballo, Fernando

Suárez-Casal, Pedro

Suarez-Garcia, Andres

Suárez-López, María José

Subaric, Drago

Subbian, Selvakumar

Subianto, Surya

Subirats, Laia

Subocz, Jan

Subramaniam, Aravinth

Subramanian, Arul

Subramanian, Rahul

Subramanian, Vignesh

Subramoniam, Ramesh

Subrin, Kévin

Sucharda, Oldrich

Suchocki, Czeslaw

Suchorzewski, Jan

Suciu, Alin 


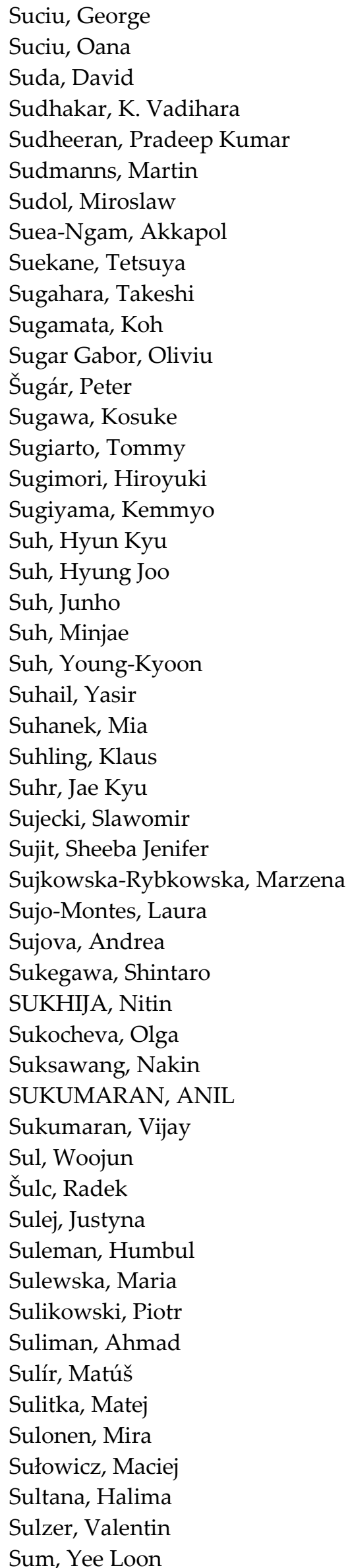

Šumak, Boštjan

Summers, Rebekah

Sümser, Kemal

Sun, Chang-Guk

Sun, Chung-Hsun

Sun, Guangyong

Sun, Guoxing

Sun, Haiyan

Sun, Haiying

Sun, Hua

Sun, Hung

Sun, I-Wen

Sun, Keye

Sun, Kunfeng

Sun, Lei

SUN, MingKuan

Sun, Peng

Sun, Rui

Sun, Shangpeng

Sun, Shengxiong

Sun, Weiqing

Sun, Wenfei

Sun, Wookyung

Sun, Xiuxuan

Sun, Yongle

Sun, Zhibin

Sun, Zhonghua

Sun, Ziheng

Sundar, Victor John

Sundararajan, Raji

Sundermann, Andreas

Sundqvist, Jan-Olov

Sundström, Aksel

Sung, Mankyu

Sung, Sangwoo

Sung, Si-Il

Sung, Tae-Eung

Sung, Wen-Chieh

Sung, Wen-Wei

Sunindijo, Riza

Sunkara, Vikram

Sünter, Indrek

Suntornnond, Ratima

Suo, Yu

Supej, Matej

Suprun, Emiliya

Sur, Sanjib

Surace, Cecilia

Surace, Rossella

Suraneni, Prannoy

Surdo, Salvatore 
Suresh, Mahima Agumbe

Suresh, Subbulakshmi

Surian, Didi

Surić, Maša

Surleva, Andriana

Surmick, David

Surugiu, Maria Claudia

Suryadevara, Nagender Kumar

Suryadevara, Vidyani

Suryanto, Bryan

Suryavanshi, Santosh

Susanj Cule, Ivana

Susek, Waldemar

Suslov, Konstantin

Susnea, Ioan

Sussman, Dafna

Sutej, Ivana

Sutherland, James

Sutin, Alexander

Sütő, Zoltán

Sutowska, Marzena

Suwada, Tsuyoshi

Suwała, Wojciech

Suwan, Keittisak

Suwardi, Ady

Suzuki, Anna

Suzuki, Arata

Suzuki, Hiroaki

Suzuki, Jun

Suzuki, Koutarou

Suzuki, Masako

Svabova, Lucia

Svaizer, Piergiorgio

Švajlenka, Jozef

Svartengren, Magnus

Svatek, Vojtech

Svatos, Jakub

Svec, Ivan

Svecko, Rajko

Sven, Gotovac

Svendsen, Hallvard F.

Svendsen, Morten Bo Søndergaard

Svensson, Ann

Svensson, Carl-Magnus

Sverbilov, Victor

Svetlik, Jan

Svetlík, Jozef

Svetovoy, Vitaly B.

Svilainis, Linas

Svoboda, Jiří

Svobodová, Libuše
Svorobej, Sergej

Svyatoslav, Durlevich

Swacha, Jakub

Swaileh, Wassim

Swails, Nahid

Swain, Michael V.

Swan, Pamela D.

Swanson, Kylie

Świątek, Łukasz

Swiatkowska, Zaneta

Świć, Antoni

Swider, Jerzy

Świderek, Katarzyna

Swiderska-Chadaj, Zaneta

Świderski, Waldemar

Święch, Łukasz

Świercz, Mirosław

Świercz, Rafał

Świerczyńska, Aleksandra

Świerk, Dariusz

Świerkosz, Krzysztof

Świetlicka, Izabela

Świetlik, Dariusz

Swinarew, Andrzej

Swindle, Andrew

Świt, Grzegorz

Świtońska, Milena

Syaekhoni, M. Alex

Syafrudin, Muhammad

Sycheva, G.A.

Sydlowski, Lukasz

Sydoruk, Oleksiy

Syed, Qurban Ali Shah

Syed, Sana

Sygouni, Varvara

Sykłowska-Baranek, Katarzyna

Sýkora, Jan

Sylvia IV, J. J.

Symeonaki, Eleni G.

Symeonidis, Andreas L.

Symons, Jennifer

Synoradzki, Karol

Syromyatnikov, Mikhail Y.

Syrovy, Tomas

Systä, Kari

Szabelski, Jakub

Szabó, György

Szabo, Lorand

Szabó, Szilárd

Szabolcsi, Róbert

Szachogluchowicz, Ireneusz 
Szafarczyk, Anna

Szafran, Jacek

Szafranek-Nakonieczna, Anna

Szafraniec, Małgorzata

Szafranko, Elżbieta

Szafrański, Marek

Szajerski, Piotr

Száková, Jiřina

Szala, Miroslaw

Szaleniec, Maciej

Szałowski, Karol

Szamałek, Krzysztof

Számel, László

Szark-Eckardt, Mirosława

Szaszák, György

Szata, Mieczysław

Szczepaniak, Piotr S.

Szczepaniak, Włodzimierz

Szczepankowski, Pawel

Szczepański, Marcin

Szczepiński, Jacek

Szczerska, Malgorzata

Szcześniak, Anna

Szcześniak, Paweł

Szczęsny, Szymon

Szczucka-Lasota, Bożena

Szczuko, Piotr

Szczypinski-Sala, Wojciech

Szczypiorski, Krzysztof

Szejba, Daniel

Szekely, Gyorgy

Szeląg, Bartosz

Szeląg, Maciej

Szeleszczuk, Łukasz

Szeliga, Monika

Szepvolgyi, Janos

Szermer, Michal

Szewczuk-Karpisz, Katarzyna

Szewczyk, Bartłomiej

Szewczyk, Roman

Szidarovszky, Tamás

Sziebig, Gabor

Szielasko, Klaus

Szilagyi, Henriette

Szilágyi, Istvan

Szkaradkiewicz, Andrzej

Szmagliński, Jacek

Szmelter-Jarosz, Agnieszka

Szmigiera, Elżbieta

Szoka, Łukasz

Szold, Amir
Szombara, Stanisław

Szóstak, Mariusz

Szott, Szymon

Szperlich, Piotr

Szpyrka, Ewa

Sztanke, Krzysztof

Sztanke, Małgorzata

Sztubecka, Małgorzata

Szukalski, Adam

Szul, Tomasz

Szulc, Karolina

Szulczyński, Bartosz

Szulwic, Jakub

Szumera, Magdalena

Szumowski, Maksymilian

Szumski, Oskar

Szurgacz, Dawid

Szurlej, Adam

Szutkowski, Kosma

Szwaja, Stanisław

Szwajca, Anna

Szwajka, Krzysztof

Szweda, Piotr

Szwedziak, Katarzyna

SZYBIŃSKI, BOGDAN

Szyluk, Karol J.

Szymanda, Jaroslaw

Szymaniec, Krzysztof

Szymanowska, Urszula

Szymanowski, Mariusz

Szymańska-Pulikowska, Agata

Szymański, Grzegorz

Szymanski, Jerzy R.

Szymański, Kaziemierz

Szymczak, Tadeusz

Szymczyk, Magdalena

Szymczyk, Piotr

Szymenderski, Jan

Szymiczek, Małgorzata

Szymon, Kugler

Szymona, Karolina S.

Szypuła, Bartłomiej

Szyszka, Jerzy

Tabacaru, Aurel

Tabaković, Amir

Tabandeh, Shahin

Tabaran, Flaviu

Tabata, Tomohiro

Tabatabaeipour, Morteza

Tabei, Fatemehsadat

Taber, Christopher B. 
Tabish, Tanveer

Taborri, Juri

Tabuchi, Masashi

Taddei, Tommaso

Tade, Moses

Tadesse, Girmaw Abebe

Tadic, Bosiljka

Tadmor, Rafael

Tadyszak, Krzysztof

Taebi, Amirtahà

Tae-Hwee Lee, Taylor

Taeihagh, Araz

Tafarojnoruz, Ali

Tafidis, Pavlos

Taflove, Allen

Tagarakis, Aristotelis

Tagawa, Hiroshi

Tagawa, Toshio

Taghipour, Armin

Tagliabue, Lavinia Chiara

Tagliaferro, Alberto

Tagliavini, Giuseppe

Tago, Kenji

Taguchi, Yoshihiro

Taha, Hussameldin

Tahami, Hoda

Taher Azar, Ahmad

Taheri, Hossein

Taheri, Pooya

Taheri, Shima

Taherzadeh, Mohammad

Tahmasebi, Pejman

Tahmasebinia, Faham

Tahmasian, Sevak

Tahmassebi, Amirhessam

Tahmooresnejad, Leila

Taiar, Redha

Taibi, Amel

Taichi Furukawa, Taichi

Taidi, Behnam

Tajiki, Mohammad Mahdi

Tak, Byungchul

Takács, Erzsebet

Takács, Sándor

Takada, Akihiko

Takahashi, Akihisa

Takahashi, Kazutaka

Takahashi, Keisuke

Takahashi, Ken

Takahashi, Shuntaro

Takahashi, Susumu
Takahashi, Toshiyuki

Takamatsu, Jun

Takano, Hajime

Takarics, Béla

Takata, Kazutaka

Takayuki, Tsukui

Takebe, Hiromichi

Taked, Takayuki

Takeda, Kotaro

Takeda, Ryo

Takeda, Toshinobu

Takeda, Yukio

Takemoto, Shinji

Takemura, Haruo

Taketomi, Shinya

Takeuchi, Masaru

Takewaka, Satoshi

Takeyama, Akinori

Takino, Junichi

Takizawa, Shinya

Takosogulu, Jakub Emanuel

Takuma, Takashi

Talaghir, Laurentiu-Gabriel

Talakoub, Omid

Talebi, Saeed

Taler, Dawid

Talhinhas, Pedro

Talianu, Camelia

Talimian, Ali

Tallapally, Venkatesham

Tallarico, Marco

Tallents, Greg J.

Tallman, Tyler N.

Talpey, Scott W.

Țălu, Ștefan

Talukdar, Saurav

Talukder, Poulami

Tam, Gary K. L.

Tam, Roger Y.

Tama, Bayu Adhi

Tamaddun, Kazi

Tamagnone, Mario

Tamaian, Radu

Tamarin, Ollivier

Tamás, Levente

Tamás, Péter

Tamazin, Mohamed

Tamer, Aykut

Tamil, Lakshman

Tamm, Tarmo

Tammaro, Umberto 
Tamošaitienė, Jolanta

Tampakas, Vasilis

Tampieri, Francesco

TAMSSAOUET, Ferhat

Tamus, Zoltán Ádám

Tan, Bo

Tan, Chee-Keong

Tan, Chin Yaw

Tan, Hua

Tan, Lee

Tan, Phan Xuan

Tan, Rex Xiao

Tan, Sheng

Tan, Xipeng

Tan, Yiyu

Tan, Zhengguo

Tanabe, Masayuki

Tanaka, Jiro

Tanaka, Kenji

Tanaka, Miyako

Tanaka, Naoro

Tanaka, Satoshi

Tanaka, Tim Hideaki

Tanaka, Yohsuke

Tanambell, Hartono

Tanase, Corneliu

Tanda, Giovanni

Tanda, Maria Giovanna

Taneja, Mohit

Tang, Chao-Wei

Tang, Cheng-Ming

Tang, Jijun

Tang, Lingfei

Tang, Longteng

Tang, Peter H.

Tang, Shih-tsang

Tang, Xin

Tangaro, Sonia

Tango, Fabio

Taniguchi, Akira

Taniguchi, Takuya

Tannant, Dwayne

Tanner, David Colin

Tanniru, Mohan

Tanno, Takenori

Tanoubi, Issam

Tanskanen, Kari

Tanvir, Shams

Tanzi, Maria Cristina

Tao, Bing

Tao, Chenqi
Tao, Feifei

Tao, Jian

Tao, Jin

Tao, Jing

Tao, Ran

Tao, Wei

Tao, Xuyuan

Tao, Yunwen

Taoka, Yousuke

Ťapák, Peter

Tapete, Deodato

Tapia-Fernandez, Santiago

Tapie, Laurent

Tappa, Karthik

Tappia, Paramjit

Tar, Jozef

Tara, Kam

Tarabanko, Valery E.

Țarălungă, Dragoș

Taran, Elena

Taran, Narges

Tarannum, Mubin

Tarantino, Laura

Țăranu, Nicolae

Tararova, Olga

Taraseviciene, Zivile

Tarasiuk, W.

Tarasov, Dmitry

Tarca, Radu

Tarczewski, Romuald

Targiel, Krzysztof

Tariq, Mohammad

Tarkowská, Danuše

Tarkowski, Maciej

Tarnas, Jacek

Tarraste, Marek

Tarrés, Quim

Tartaglia, Roberto

Taruta, Seiichi

Tascón, Alberto

Tashakori, Shervin

Tashima, Toshihiko

Tasinato, Nicola

Tasoulis, Sotiris

Tassone, Sonia

Tassou, Chrysoula

Tatakis, Dimitris

Tatar, Mihai Olimpiu

Tatara, Robert

Tatara, Tadeusz

Tateishi-Karimata, Hisae 
Tatlas, Nicolas-Alexander

Tatsuke, Tsuneyuki

Tatsuoka, Hirokazu

Tatsushi, Nishi

Tatters, Avery O.

Tatullo, Marco

Tatzko, Sebastian

Tauš, Peter

Tavani, Cinzia

Tavanti, Francesco

Távara, Luis

Tavares, João Manuel R. S.

Tavares, Sérgio M. O.

Tavassoly, Iman

Tavassoti, Pejoohan

Tavelli, Maurizio

Tavernier, Wouter

Tavolato, Paul

Tawalbeh, Loai

Tawfiq, Kotb

Tawose, Olamide Timothy

Tay, Jian Wei

Taylor, Adam

Taylor, Alan

Taylor, Nigel George

Taynitskiy, Vladislav

Tayurskii, Dmitrii

Tayyab, Muhammad

Tbaileh, Ahmad

Tchagang, Alain

Tchawou Tchuisseu, Batista Eder

Tchemisova, Tatiana

Tcherdyntsev, Victor V.

Tcvetkov, Pavel

Te Nijenhuis, Jan

Teacă, Carmen Alice

Tecelão, Diogo

Tedeschi, Paola

Tedesco, Giacomo

Tedesco, Roberto

Tefelski, Dariusz

Tegowski, Jaroslaw

Teh, Kah Chan

Teh, Muy-Teck

Teh, Ying Khai

Tehranchi, Amirhossein

Tehrani, Rouzbeh

Teimourian, Amir

Teischinger, Alfred

Teixeira Domingues, José Pedro

Teixeira, António
Teixeira, Joao Amaral

Teixeira, Senhorinha

Tejedor, Blanca

Tekes, Ayse

Tekes, Coskun

Teklemariam, Aron

Telada, Souichi

Telichko, Arsenii

Teloglou, Elias

Telyakovskiy, Aleksey

Tempel, Philipp

Tempfer, Herbert

Templin, Tomasz

Temprano, Israel

Ten Bosch, L.F.M.

Tenace, Valerio

Teng, Kah Hou

Teng, Yue

Tenkumo, Taichi

Tenore, Gianluca

Tenorio, Jose Antonio

Tenorio, Victor Octavio

Teo, How Wei Benjamin

Teo, Jeremy

Teo, Rodney Swee Huat

Teodora-Mihaela, Iconomescu

Teodorescu, Florina

Teoh, Yu-Yao

Teparić, Renata

Tepljakov, Aleksei

Tera, Masayuki

Tera, Melania

Teramoto, Shinji

Teramoto, Yoshikuni

Terebes, Romulus

Teresa Pérez-Gracia, María

Terme, Nolwenn

Terner, Mathieu

Terrazas Angulo, German

Terrien, Ryan

Terroso, Fernando

Terroso-Saenz, Fernando

Terry, Patricia

Terzaghi, William Bryan

Tesar, Jiri

Teschner, Tom-Robin

Teslic, Nemanja

Teso-Fz-Betoño, Daniel

Tesoriero, Ricardo

Tessarolo, Marta

Tesser, Riccardo 
Tessitore, Antonio

Tesson, Sylvie

Testa, Alessandro

Testa, Gabriel

Testarelli, Luca

Testoni, Nicola

Teti, Luca

Teti, Roberto

Tetougueni, Cyrille Denis

Tetsuya, Kawachi

Texido Bartes, Robert

Težak, Denis

Thabtah, Fadi

Thai, Duc-Kien

Thakker, Dhaval

Thakor, Nitish

Thakur, Ashutosh

Thakur, Nirmalya

Thakur, Subhasis

Thalfeldt, Martin

Thangavel, Chellappagounder

Thanh, Kien Nguyen

Thapa, Rajan

Thapliyal, Vivek

Thavamani, Aravind

Thebault, Karim

Thekkinkattil, Dinesh K.

Theodorakeas, Panagiotis

Theodorakis, Panagiotis

Theodoros, Kosmanis

Theodossiou, Nicolaos

Theodoulidis, Nikos

Theoharis, Babanatsas

Theres Baby, Tessy

Therriault, Daniel

Thevenot, Paul T.

Thiagarajan, Ganesh

THIBAUDON, Michel

Thibault, Simon

Thiel, Teresa

Thiery, Jean Marc

Thimont, Yohann

Thirunavukarasu, Deepak

Thiruvengadam, Muthu

Thissen, Peter

Thoden Van Velzen, Ulphard

Thomä, Marco

Thomas, Adam

Thomas, Carlos

Thomas, Gray C.

Thomas, Jeremy N.
Thomas, Morgan L.

Thomas, Peter

Thomas, Sean

Thomas, Yannis

Thompson, Jonathan M.

Thompson, Pamela

Thompson, Roberta

Thomsen, Jesper S.

Thomsen, Jonas Bjerg

Thorleifson, Harvey

Thorleuchter, Dirk

Thrinath, Reddy Ramireddy

THUC, TRAN VAN

Thulasiraman, Krishnaiyan

Thummuri, Dinesh

Thyele, Geoffrey

Tiainen, Jonna

Tiakas, Eleftherios N.

Tian, Gui Yun

Tian, Kun Viviana

Tian, Liang

Tian, Lin

Tian, Yun

Tian, Zhenhua

Tiba, Dan

Tibaut, Andrej

Tibbetts, Katharine Moore

Tiberi, Gianluigi

Tiberius, Victor

Tiboaca, Daniela

Tiboni, Monica

Ticha, Helena

Tiels, Koen

Tien, Chung-Hao

Tieslau, Margie A.

Tietze, Thomas

Tigani, Domenico

Tiggelaar, Roald M.

Tigrini, Andrea

Tihkonov, Vladimir

Tiisanoja, Antti

Tiitta, Markku

Tijan, Edvard

Tijani, Saheed

Tijus, Charles

Tikhomirova, Irina

Timar, Gabor

Time, Rune W.

Timm, Ingo J.

Timmers, Ralph

Timoney, Joseph 
Timoshenko, Janis

Timothy, Jithender J.

Ting, Jeffrey M.

Ting, Yung

Tinni, Ali Ousseini

Tintelecan, Marius

Tinti, Francesco

Tiong, Leslie Ching Ow

Tiow, Ooi Kim

Tipireddy, Ramakrishna

Tiplea, Ferucio Laurentiu

Tipre, Meghan

Tirado, Diego F.

Tirillo, Jacopo

Tirilly, Pierre

Tiringer, Ursa

Tischler, Dirk

Tishchenko, Victor

Tisovský, Pavol

Tita, Ovidiu

Titov, Aleksei A.

Titova, Ekaterina S.

Titti, Giacomo

Titze, Jean

Tiwale, Nikhil

Tiwari, Ashutosh

Tizek, Linda

Tkach, Itshak

Tkachev, Stepan

Tkaczuk, Cezary

Tkavc, Rok

Tlach, Vladimír

To, Peter

Toader, Dumitru

Tobarra, Llanos

Többens, Daniel

Tobina, Takuro

Tobis, Slawomir

Tobiszewski, Marek

Tocmo, Restituto

Toczylowska-Maminska, Renata

Todaro, Luigi

Todde, Giuseppe

Todhunter, Luke

Todhunter, Paul E.

Todorov, Filip

Todorov, Miroslav

Todorov, Svetoslav Dimitrov

Todros, Silvia

Toedebusch, Christine

Toelle, Brian
Toffano, Zeno

Toffoli, Andrea

Tofil, Arkadiusz

Tognetti, Alessandro

Tohme, Fernando

Tohmura, Shin-ichiro

Tojo, Adrián F.

Tojo, Naoki

Tokarska, Magdalena

Tokarz, Krzysztof

Tokatlidis, Ioannis

Toker, Onur

Tokunaga, Eiji

Tokura, Yasuhiro

Toldrá, Fidel

Tolias, Ilias

Toliyat, Hamid A.

Tolkou, Athanasia

Tolleson, Doug

Tolou Kian, Mohammad J.

Tolstoy, Peter M.

Tolstykh, Tatyana

Tolvanen, Jarkko

Toma, Ionut Ovidiu

Toma, Milan

Toma, Pierluigi

Toma, Radu Bogdan

Tomai, Eleni

Tomaiuolo, Michele

Tomar, Anuradha

Tomás, Antonio Espín

Tomas, Séverine

Tomašegović, Tamara

Tomasello, Barbara

Tomasic, Nenad

Tomasiello, Stefania

Tomášková, Hana

Tomastik, Jan

Tomasz, Falborski

Tomasz, Rymarczyk

Tomaszewska, Agnieszka

Tomaszewska, Justyna

Tomaszewska-Hetman, Ludwika

Tomaszewski, Daniel

Tomaszuk, Dominik

Tomat, Valentina

Tomažič, Simon

Tomczak, Janusz

Tomczak, Michał

Tomczak, Szymon

Tomczyk, Arkadiusz 
Tomczyk, Krzysztof

Tomczyk, Łukasz

Tomei, Alice

Tomer, Vijay K.

Tomescu, Mirela Cleopatra

Tomic, Hrvoje

Tomiczek, Krzysztof

Tomikawa, Ko

Tomioka, Satoshi

Tomiyama, Shingo

Tomkin, Gerald

Tomków, Jacek

Tomkowski, Robert

Tomm, Jens

Tomoaki, Karaki

Tomori, Hiroki

Tomoyoshi, Nishimura

Tomporowski, Andrzej

Tonacci, Alessandro

Tonda, Alberto

Tonetti, Lorenzo

Tong, Kenneth

Tong, Michael Chi Fai

Tong, Van-Canh

Tong, Xinjie

Tonin, Stefania

Tonkin, Emma

Tonni, Ingrid

Tono, Kensuke

Tönsmann, Max

Tootkaboni, Mazdak

Topala, Ionut

Topic, Danijel

Topic-Popovic, Natalija

Topoglidis, Emmanuel

Topolář, Libor

Topolinski, Szymon

Topor, Marcel

Topp, Elin Anna

Topsakal, Mehmet

Torchio, Marcello

Tordeux, Antoine

Toreini, Ehsan

Torigoe, Kanjiro

Torija Martinez, Antonio J.

Torley, Peter

Tornabene, Francesco

Tornari, Vivi

TORNATORE, Vincenza

Tornow, Florian

Torok, Adam
Toroker, Maytal Caspary

Toroń, Bartłomiej

Toronov, Vladislav

Torp, Olav

Torralba, Marta

Torre Toledano, Doroteo

Torre, Maria Luisa

Torrecillas, Cristina

Torreglosa, Juan P.

Torregrosa, Adrian

Torres Arriaza, José Antonio

Torres Marques, Antonio

Torres Vaamonde, José Enrique

Torres Vega, Maria

Torres, Ana

Torres, Arnau Mir

Torres, Javier Martínez

Torres, Maria Leilani

Torres, Miguel Garcia

Torres, Pedro M. B.

Torres, Ricardo Da Silva

Torres, Roque

Torres, Rosa

Torres-Ferrera, Pablo

Torres-Gonzalez, Arturo

Torres-Lagares, Daniel

Torres-Sánchez, Irene

Torres-Sospedra, Joaquín

Torri, Emanuele

Torri, Luisa

Torri, Marco Danilo Claudio

Torrico, Damir D.

Torrisi, Geraldine

Torrisi, Vincenza

Torsello, Loredana

Torti, Emanuele

Torti, Valeria

Tortia, Cristina

Tortonesi, Mauro

Tortosa, Dídac D.

Tosanovic, Natasa

Toscani, Anita

Tostado-Véliz, Marcos

Totan, Alexandra Ripszky

Totaro, Michael W.

Totero Gongora, Juan Sebastian

Toth, Andras Jozsef

Toth, Balazs

Toth, Frantisek

Toth, Travis D.

Tóth, Zoltán 
Tóth, Zsolt

Tothova, Csilla

Totta, Pierangela

Tougait, Olivier

Touloupaki, Eleftheria

Townsend, Kathy

Toyoma, Tadashi

Toyota, Hirofumi

Toyoura, Masahiro

Tozer, Peter

Tozza, Silvia

Tozza, Stefano

Trach, Roman

Trackemas, Jack

Traffano-Schiffo, María Victoria

Trainiti, Giuseppe

Trainor, Patrick

Trakadas, Panagiotis

Trakakis, George

Trakas, Dimitris

Tralli, Velio

Tramelli, Anna

Trammell, Samuel

Tran, Bach

Tran, Binh Q.

Tran, Dat

Tran, Duc-Thien

Tran, Hoang

Tran, Jonathan

Tran, Kim Phuc

Tran, Le Vu

Tran, Quynh

Tran, Van-Thai

Tranca, Dumitru Cristian

Tranfo, Giovanna

Tranoris, Christos

Tranquillo, Elisabetta

Transtrum, Mark K.

Traore, Mamadou Kaba

Trapanese, Marco

Trapani, Ferdinando

Trappe, Wade

Traub, Lance

Travassos, Bruno

Travieso-González, Carlos

Travieso-Gonzalez, Carlos M.

Trávničková, Tereza

Trcek, Janja

Trdan, Stanislav

Trebinski, Radosław

Treideris, Marius
Trematerra, Amelia

Trematerra, Pasquale

Trenchev, Georgi

Trentadue, Francesco

Trépout, Sylvain

Tres, Alba

Tretiakov, Konstantin

Tretiakov, Konstantin V.

Treutler, Kai

Trevisan, Andrea

Trevlakis, Stylianos E.

Tri Phung, Quoc

Triantafillou, Thanasis

Triantafyllou, Ioannis

Triantafyllou, Savvas

Triantis, Dimos A.

Triantou, Marianna

Trichakis, Ioannis

Tridello, Andrea

Trifiletti, Vanira

Trifina, Lucian

Trifonova, Tatiana A.

Trigeassou, Jean-Claude

Trigili, Emilio

Trigo, Juan Francisco

Trigo-Rodríguez, Josep M.

Trihinas, Demetris

Trim, Peter R. J.

Trimigno, Alessia

Trincone, Antonio

Trindade, Ana

Trindle, Carl

Trinh, Minh Hoang

Trinh, Minh-Chien

Trinkler, Laima

Tripathi, Diwaker

Tripathi, Madhulika

Tripathy, Sunil Kumar

Tripicchio, Paolo

Trisolino, Giovanni

Tristant, Damien

Trivedi, Rahul

Trivellin, Nicola

Trkulja, Bojan

Trochimczuk, Roman

Trogu, Antonio

Trohman, Richard

Troia, Adriano

Troiani, Enrico

Trojanowska, Monika

Trojner-Bregar, Andreja 
Tronstad, Russell

Tropina, Albina

Tropmann-Frick, Marina

Trotta, Gianluca

Troussas, Christos

Trovalusci, Patrizia

Trovati, Marcello

Trovato, Maria Rosa

Trubiani, Oriana

Truchot, Benjamin

Trujillo, Francisco Javier

Trukhanov, Alex V.

Trull, Óscar

Truniger, Verónica

Trusiak, Maciej

Trybek, Paulina

Trybuła, Zbigniew

Tryk, Donald

Trynchuk, Viktor

Tryznowski, Mariusz

Trzaska, Jacek

Trzaskowska, Monika

Trzcinski, Jerzy

Trzeciak, Anna

Trzepiecinski, Tomasz

Trzmiel, Grzegorz

Tsagaris, Apostolos

Tsai, Ang-Chen

Tsai, Cheng-Fa

Tsai, Chi-Chin

Tsai, Chih-Fong

Tsai, Hsieh-Fu

Tsai, Isheng

Tsai, Kang-Ting

Tsai, Kuang-Chung

Tsai, Meng-Han

Tsai, Meng-Hsiu

Tsai, Mi-Ching

Tsai, Ming-yi

Tsai, Pang-Wei

Tsai, Pei-Hsuan

Tsai, Shih-Chin

Tsai, Yao-Te

Tsai, Yu-Ting

Tsai, Zuo-Min

Tsakalidis, George

Tsakanikas, Panagiotis

Tsakirakis, Eleftherios

Tsaklis, Panagiotis

Tsalagkas, Dimitrios

Tsalidis, Georgios Archimidis
Tsaltas, Dimitris

Tsamatsoulis, Dimitris

Tsang, Yung Po

Tsangas, Michail

Tsangouri, Eleni

Tsangrassoulis, Aris

Tsao, Chung Chen

Tsao, Hoi Nok

Tsao, Lung-Chuan

Tsaopoulos, Dimitrios

Tsarev, Andrei

Tsarkova, Larisa A.

Tsaroucha, Efthymia

Tsarouhas, Vasilios

Tsatsaris, Andreas

Tsau, Jyun-Syung

Tsaur, Ruey-Chyn

Tse, Daniel W. K.

Tsehaye, Misgina Tilahun

Tseluikin, Vitaly

Tseng, Kuang-Wen

Tseng, Ming-Lang

Tseng, Shu-Ming

Tseng, Shuo-Yen

Tseng, Tsun-Ming

Tseng, Wei-Lung

Tseng, Yuan-Wei

Tseng, Yuen-Hsien

Tsentalovich, Yuri P.

Tsepelev, Vladimir

Tsiatsikas, Zisis

Tsige, Meaza

Tsigopoulos, Andreas D.

Tsiligkaridis, John (Ioannis)

Tsimeas, Panagiotis

Tsimpanogiannis, Ioannis N.

Tsinos, Christos G.

Tsionas, Mike

Tsionas, Mike G.

Tsipouras, Markos G.

Tsironi, Fanny

Tsironi, Theofania

Tsiropoulou, Eirini Eleni

Tsitsifli, Stavroula

Tso, Chi Yan

Tso, Edwin

Tsogka, Chrysoula

Tsolas, Ioannis

Tsotsis, Theodore T.

Tsotsos, Lia E.

Tsou, Nien-Ti 
Tsoulias, Nikos

Tsoumanis, Georgios

Tsoutsanis, Elias

Tsouvalas, Apostolos

Tsovilis, Thomas E.

Tsuboi, Tsutomu

Tsuchiya, Satoshi

Tsuchiya, Tatsuhiro

Tsuji, Hideto

Tsuji, Yasuhide

Tsujimoto, Yoshinobu

Tsujino, Noriyoshi

Tsukada, Manabu

Tsukahara, Takahiro

Tsukahara, Takamitsu

Tsukakoshi, Kaori

Tsukegi, Takayuki

Tsukushi, Satoshi

Tsuneda, Takao

Tsunenori, Mine

Tsung, Chen-Kun

Tsunoda, Hiroshi

Tsushima, Ikuo

Tsutsumi, Daizo

Tsvetanov, Christo

Tsvetkov, Nikolai

Tsvetkov, Vladimir B.

Tsyganenko, Alexey A.

Tu, Chuan-Chi

$\mathrm{Tu}$, Shu-Fen

Tubaldi, Enrico

Tubbal, Faisel

Tucan, Paul

Tucker, Gordon C.

Tucki, Karol

Tucu, Dumitru

Tudon-Martínez, Juan

Tudor, Eugenia Mariana

Tudor, Kate

Tuegeh, Maickel

Tufarelli, Vincenzo

Tufariello, Miriam

Tullberg, Cecilia

Tuluri, Francis

Tumino, Davide

Tummino, Maria Laura

Tundis, Andrea

Tundis, Rosa

Tundys, Blanka

Tunér, Jan

Tung Chong, David Wong
Tuohy, Pat

Tuovinen, Tero

Turan, Cigdem

Turanoglu Bekar, Ebru

Turchin, Ilya

Turco, Gianluca

Turconi, Laura

Turcu, Antoniu

Turek, Anna

Turek, Vojtěch

Turhanen, Petri

Turiel, Javier Pérez

Turk, Goran

Turk, Žiga

Turki, Houcemeddine

Turkiewicz, Igor Piotr

Turkoglu, Kamran

Turnbull, Marion T.

Turoń, Katarzyna

Turpin, Alex

Tursi, Antonio

Turuallo, Gidion

Turunen, Markku

Tusek, Ana Jurinjak

Tušek, Ana Jurinjak

Tutanescu, Ion

Tutueva, Aleksandra

Tutunaru, Lucian

Tuyen, Nguyen Duc

Tuz, Lechosław

Tuzimski, Tomasz

Twardowski, Paweł

Twisa, Sekela

Twitchell, Douglas

Twu, Ruey-Ching

Tyaginov, Stanislav

Tyan, Maxim

Tylewicz, Urszula

Tyliszczak, Bożena

Tylkowski, Jacek

Tyrologou, Pavlos

Tyshchenko, Oleksii

Tysiac, Pawel

Tyson, Darren R.

Tytła, Malwina

Tyutin, Marat

Tzallas, Alexandros

Tzanetakis, Giorgos

Tzanetos, Alexandros

Tzanis, Chris G.

Tzeng, Shiang-Jong 
Tziavos, Nikolaos

Tziolas, Nikolaos

Tziourtzioumis, Dimitrios N.

Tziveleka, Leto-Aikaterini

Tzortzopoulos, Patricia

Tzounis, Lazaros

Tzouvaras, Marios

Tzovenis, Ioannis

Tzschatzsch, Heiko

Tzu, Fu-Ming

Ubertini, Filippo

Ubysz, Andrzej

Uccheddu, Francesca

Uceda, Antonio Lopez

Ucgul, Mustafa

Uchida, Satoshi

Uchino, Haruto

Uchino, Kenji

Uchiyama, Kenji

Uciński, Dariusz

Udaka, Hiroko

Uddi, Mruthunjaya

Uddin Ahamed, Nizam

Uddin, Majbah

Udroiu, Razvan

Udugama, Asanga

Ueberschär, Olaf

Ueji, Rintaro

Ueng, Fang-Biau

Ueng, Shyh-Kuang

Ugail, Hassan

Ugon, Adrien

Ugrina, Marin

Uhlig, Jens

Uhríčik, Milan

Uhrig, Stephanie

Uhring, Wilfried

Ujihara, Masaki

Ukalska-Jaruga, Aleksandra

Ukrainczyk, Neven

Ukrainski, Kadri

Ukwandu, Elochukwu

Ul Haque, Adnan

Ul Huque, Md Tanvir Ishtaique

Ulacha, Grzegorz

Ulbin, Miran

Ulbrich, Dariusz

Ulewicz, Malgorzata

Ulewicz, Robert

Ullah, AMM Sharif

Ullah, Habib
Ullah, Mohib

Ullah, Zaib

Ulloa, Alvaro

Ulloa, Carlos

Ulloa, Hugo

Ulmeanu, Mihaela

Ulyashin, Alexander G.

Um, Doo-seung

Um, Jumyung

Umar Khan, Muhammad

Umayahara, Yasutaka

Umbleja, Kadri

Umbrico, Alessandro

Umemura, Akira

Umemura, Naoki

Umer, Muhammad

Umerska, Anita

Umili, Gessica

Undabeytia, Tomas

Undersander, Dan

Unger, Jakob

Ungurean, Ioan

Ungureanu, Florina

Ungureanu, Stefan

Ungurianu, Anca

Unno, Keiko

Uno, Kazuyuki

Unruh, Daniel

Unsal, Omer

Unsworth, Amanda

Unterreiner, Andreas-Neil

Unune, Deepak Rajendra

Upreti, Girish

Ur Rehman, Junaid

Ur Rehman, Masood

Ur, Soon-Chul

Úradníček, Juraj

Urakami, Noriyuki

Urashima, Kuniko

Urbain, Xavier

Urbán, Daniel

Urban, Wieslaw

Urbančič, Tilen

Urbancl, Danijela

Urbanczyk-Lipkowska, Zofia

Urbanelli, Lorena

Urbaniak, Maciej

Urbaniak, Wiesław

Urbaniec, Krzysztof

Urbano, Diana

Urbanowicz, Kamil 
Urbańska, Weronika Martyna

Urbanski, Marek

Urbański, Mariusz

Urbikain Pelayo, Gorka

Urbonas, Rolandas

Urda Muñoz, Daniel

Urgese, Gianvito

Urgessa, Girum Solomon

Uribe Quevedo, Alvaro Joffre

Urniezius, Renaldas

Uroić, Tessa

Uros, Mario

Urquiza-Aguiar, Luis

Urso, Mario

Ursu, Ioan

Urtnasan, Erdenebayar

Urwyler, Prabitha

Urzì, Clara E.

Usai Satta, Paolo

Usai, Donatella

Usamentiaga, Ruben

Usarek, Zbigniew

Ushakov, Nikolai

Ushakov, Nikolay M.

Ushakova, Elena V.

Ushida, Akiomi

Ushizima, Daniela

Uslar, Mathias

Usman, Muhammad

Ustun, Taha Selim

Usui, Kenji

Usui, Yoichi

Utgikar, Vivek

Utkin, Andrei

Utrilla-Manso, Manuel

Utsunomiya, Toru

Uusitalo, Topi

Uva, Giuseppina

Uwe, Franz

Uyeno, Yutaka

Uykur, Ece

Uzhga-Rebrov, Oleg

Uzochukwu, Benedict M.

V Ivanov, Alexander

V. González López, Cynthia

Vacareanu, Radu

Vacca, Gaetano

Vaccarezza, Mauro

Vaccaro, Carmela

Vaccaro, John C.

Vach, Kirstin
Vácha, Radim

Vaclav, Kotlan

Václav, Štefan

Václavíková, Natália

Vadahanambi, Sridhar

Vaezi, Mojtaba

Vafapour, Zohreh

Vafiadis, Kyriakos

Vaghei, Yasaman

Vagiona, Dimitra G

Vagionas, Christos

Vágner, Anikó

Vagnon, Federico

Vahabi, Henri

Vahdat, Vahab

Vahdati, Maria

Vahidi, Hossein

Vaiana, Nicolò

Vaiana, Rosolino

Vaida, Calin

Vaidogas, Egidijus Rytas

Vaimann, Toomas

Vaitiekūnas, Mantas

Vaitkus, Saulius

Vajda, Istvan

Vajedian, Sanaz

Vaks, Vladimir

Vala, Jiri

Valagussa, Andrea

Valais, Ioannis

Valant, Matjaz

Valcárcel-Aguiar, Beatriz

Valcic, Marko

Valčić, Sanjin

Valderrama Sakuyama, Carlos

Valdés-Baizabal, Catalina

Valdez, Manuel Travassos

Valeev, Dmitry

Valencia, Luis David Avendaño

Valente Da Silva Couto, Maria João

Valente, Alessio

Valente, Nicola Alberto

Valentin, VLADUT

Valentini, Pier Paolo

Valentini, Roberto

Valentino, Roberto

Valentová, Kateřina

Valentukeviciene, Marina

Valenzuela, Hector

Valenzuela, Loreto

Valerga Puerta, Ana Pilar 
Valerio, Adriano

Valerio, Giulia

Valero-Mas, Jose J.

Valero-Ramón, Zoe

Valero-Romero, María José

Väli, Germo

Valiente, David

Valigi, Maria Cristina

Valipoor, Shabboo

Valipour, Mohammad

Vallavoju, Nandini

Vallejo, Javier P.

Valles-Rosales, Delia J.

Vallet, Valérie

Valletta, Alessio

Valletta, Rosa

Vallez, Noelia

Vallianatos, Filippos

Vallicelli, Elia Arturo

Valogianni, Konstantina

Valor, Enric

Valouch, Viktor

Valous, Nektarios

Valov, Nikolay

Valova, Irena

Valsaraj, Amithraj

Valsesia, Diego

Valvano, Stefano

Valverde, Alfredo

Valverde, Raul

Valverde, Victor

Valverde-Albacete, Francisco J.

Valvo, Paolo S.

Vamanu, Emanuel

Van Ballegooijen, Adriana J.

Van Ballegooijen, Hanne

Van Belle, Lucas

Van Bennekom, Wouter P.

Van Beuningen, Henk

Van Buer, Nicholas

Van Buiten, Charlene

Van De Craen, Piet

Van Den Akker, Ramon

Van Den Burg, Sander W. K.

van der Ham, Ineke

Van Der Pol, Carla W.

Van Der Putten, Jolien

Van Der Sande, Tom P. J.

Van Der Sommen, Fons

Van Der Velde, Enno

Van Der Wal, C. Natalie
Van Der Zwaard, Stephan

Van Dinther, Clemens

Van Driel, Willem D.

Van Driessche, Alexander

Van Eijck, Lambert

Van Eijnatten, Maureen

Van Gasselt, Stephan

Van Goeverden, Kees

Van Grevenynghe, Julien

Van Haaren, Jan

Van Hamme, David

Van Hoecke, Karen

Van Hoecke, Sofie

Van Hulle, Marc

Van Laarhoven, Twan

Van Laerhoven, Kristof

Van Le, Hanh Thi

Van Leeuwen, David A.

Van Leeuwen, Hans

Van Lier, Ben

Van Paassen, Leon

Van Rossem, Steven

Van Rossom, Sam

Van Steenberge, Geert

Van Thanh, Huynh

Van Thor, Jasper J.

Van Wallendael, Glenn

Van Walsum, G. Peter

Van Wyk, Niel

Van Wymelbeke, Virginie

Van Zanten, Malou

Vanali, Marcello

Vandeghinste, Vincent

Vanden Eynde, Jean Jacques

Vandenberghe, Stefaan

Vanderelst, Dieter

Vandoren, Bram

Vanetik, Natalia

Vangorp, Peter

Vaňková, Markéta

Vannocci, Pietro

Vanoli, Maristella

Vapnik, Yevgeny

Váradi, Györgyi

Varadinov, Maria José

Varady, Geza

Varando, Gherardo

Vardaki, Martha

Vardanega, Tullio

Vardasca, Ricardo

Varde, Aparna 
Varela, Ángel

Varela, Maria Leonilde Rocha

Varevac, Damir

Varga, Pal

Varga, Tamas

Vargas, Javier

Vargo, Andrew

Variny, Miroslav

Varlamis, Iraklis

Varnava, Kyriakos

Varo-Martinez, Marta

Varotsos, George K.

Varotsos, Panayiotis

Varrà, Maria Olga

Varrella, Stefano

Varriale, Luisa

Varvani-Farahani, Ahmad

Varvaresou, Athanasia

Vasandani, Paresh

Vasar, Cristian

Vaščák, Ján

Vasconcelos, Helena Cristina

Vasconcelos, Mário R.

Vasconcelos, Teresa Maria Pinto Coelho

Amado

Vaseashta, Ashok

Vaselli, Orlando

Vashishtha, Ashish

Vashurin, Artur

Vasilache, Simona

Vasilakos, Athanasios

Vasile, Francesca

Vasileiou, Ismini

Vasilescu, Mircea Dorin

Vasilevich, Anastasia Vital'evna

Vasilevsky, Nicole

Vasiliades, Lampros

Vasiliades, Michalis A.

Vasiliev, Mikhail

Vasiliu, Daniel

Vasiljević, Goran

Vasilyev, Roman

Vasjanov, Aleksandr

Vasmara, Ciro

Vassallo, Amy J.

Vassallo, Antonio

Vassant, Simon

Vassilakis, Costas

Vassilev, Nikolay G.

Vassiljeva, Kristina

Vassilopoulou, Isabella
Vasta, Salvatore

Vatanpour, Nahid

Vatin, Nikolai

Vatolkina, Natalia

Vattepu, Ravi

Vautz, Wolfgang

Vaverkova, Magdalena

Vaverková, Magdalena Daria

Vavourakis, Vasileios

Vayas, Ioannis

Vaz De Carvalho, Carlos

Vaz, Irene Pina

Vaz, Mário

Vaz, Pedro D.

Vazana, Udi

Vazhenin, Alexander

Vaz-Moreira, Ivone

Vázquez Ardura, Aitor

Vázquez Burguete, Jose Luis

Vazquez Espinosa, Mercedes

Vázquez Polo, Francisco José

Vázquez, Carlos

Vazquez, Francisco

Vdović, Neda

Veber, Alexander

Vecchi, Veronica

Vecchiattini, Rita

Vechetti, Ivan

Veenland, Jifke

Vega, Miguel

Vega-Fuentes, Eduardo

Vegas Hernández, Jesús M.

Vega-Zamanillo, Ángel

Vegendla, Prasad

Végh, Attila Gergely

Veiga, Germano M.

Vek, Viljem

Velázquez Blázquez, José Sebastián

Velazquez, Jonathan

Velazquez-Iturbide, J. Angel

Velazquez-Perez, Jesus E.

Veldman, Arthur E. P.

VELEA, Marian Nicolae

Velev, Dimiter Georgiev

Velha, Philippe

Velić, Natalija

Velichkova, Rositsa

VELICU, Radu

Velikanov, Vladimir

Velkavrh, Igor 
Velkov, Tony

Velliscig, Lydia

Velmuzhov, Alexander

Veloso, Bruno Miguel

Velten, Andreas

Velu, Rajkumar

Velusamy, Gandhimathi

Veluvolu, Kalyana C.

Vemuru, Krishnamurthy

Venanzi, Rachele

Venanzoni, Giuseppe

Vencúrik, Tomáš

Vendik, Irina B.

Venediktov, Vladimir

Venegas, Javier Freire

Venegas, Maria

Venettacci, Simone

Vengris, Mikas

Venkannagari, Harikanth

Venkataramana, Radha A.

Venkatasubramanian, Krishna

Venkatesan, Ramachandran

Venkateswaran, Swaminath

Venkatram, Akula

Venkatraman, Sitalakshmi

Vennat, Elsa

Venning, James

Ventouras, Erricos-Chaim

Ventruti, Gennaro

Venturini, Francesca

Venugopal, Dhamodharan

Venuti, Valentina

Venuturumilli, Sriharsha

Vera García, Francisco

Vera-Pérez, Jose

Verardo, Vito

Verbelen, Tim

Verbovšek, Timotej

Verde, Francesco

Verdeja González, Luis Felipe

Verdini, Federica

Vereide, Kaspar

Veres, Csaba

Veres, Sandor

Veres, Szilvia

Verfondern, Karl

Vergara, Alessandro

Vergara, Pedro P.

Vergidis, Kostas

Vergöhl, Michael

Vergori, Luigi
Verhaevert, Jo

Verheul, Jasper

Verheyden, Sophie

Verho, Oscar

Verhoeven, Adrie

Verlinden, Jouke C.

Verly Lopes, Dercilio Junior

Verma, Amit

Verma, Amrit

Verma, Rakesh M.

Verma, Ranjit

Vermeir, Gerrit

Vermeşan, Horaţiu

Vermeulen, Marc

Verna, Alessio

Vernetti, Lawrence A.

Veronika, Szücs

Verre, Salvatore

Verros, George

Verschoof-van Der Vaart, Wouter Baernd

Vershovskii, Anton

Verso, Maria Gabriella

Verstaevel, Nicolas

Versteegh, Marijn

Verstraten, Tom

Vértesy, Gábor

Vertruyen, Benedicte

Verzak, Željko

Vescovi, Dalila

Vesin, Jean-Marc

Vespri, Vincenzo

Vessio, Gennaro

Véstias, Mário

Vetsov, Tsvetan

Vetyukov, Yury

Veysset, David

Veziroglu, Salih

Vezzani, Roberto

Vezzetti, Enrico

Viana, Paula

Viassone, Milena

Viatkin, Aleksandr

Viberg, Olga

Vibhute, Sandip M.

Viccione, Giacomo

Vicencio, Rodrigo Barraza

Vicente, Miguel A.

Vicente, Tania

Victor, Rico

Victor, Vijay

Vida, György 
Vidakis, Nectarios

Vidal, Jorge Maestre

Vidal, Pia M.

Vidal, Tânia

Vidal-Idiarte, Enric

Vidal-Verdú, Fernando

Vidic, Jasmina

Vidmar, Peter

Vidnerová, Petra

Vidoni, Renato

Vidovic, Bojana

Viegas, Carlos

Viegas, Olga

Vieira Lopes, Nuno

Vieira, António

Vieira, Taian M.

Viel, Guido

Vien, Quoc-Tuan

Viennot, Stéphane

Viggiano, Annarita

Vigil, Miguel

Vignali, Valeria

Vignoli, Giulio

Viitala, Raine

Vijaya, Raghavan

Vijulie, Iuliana

Vikulina, Anna

Vila Sobrino, Xosé Antón

Vila, Maria Cristina C.

Vilardi, Giorgio

Vilaseca, Fabiola

Vilasi, Michel

Vilău, Radu

Vilà-Valls, Jordi

Vilches, Teresa Berdugo

Vilé, Gianvito

Vilenchik, Dan

Villa, Andrea

Villa, Matteo

Villafaina, Santos

Villagrá, Victor

Villalba, Javier

Villani, Claudio

Villani, Marco

Villani, Maria Luisa

Villanueva, Manuel Vargas

Villanueva-Llauradó, Paula

Villasmil, Willy

Villecco, Francesco

Villemur, Thierry

Villone, Massimiliano Maria
Vilović, Marino

Viman, Liviu

Viña, Jaime

Vincenti, Flaminia

Vincenzi, Loris

Vinci, Giuliana

Vinel, Alexander

Vinh, Nguyen Quang

Vining, Kyle Holmberg

Vinnik, Denis

Vinogradov, Sergey

Vinogradov, Sergey L.

Vinte, Claudiu

Vinuesa, Ricardo

Viola, Fabio

Violante, Maria Grazia

Violetta Sokoła-Szewioła, Violetta

Viollet, Stéphane

Viprey, Fabien

Viquez, Oscar A.

Virag, Zoltan

Virdee, Bal

Virgala, Ivan

Virgolino, Ana

Viricelle, Jean Paul

Virkki, Johanna

Virlanuta, Florina

Virsile, Akvile

Virtanen, Heikki

Virto, Leire

Virtuani, Alessandro

Viscito, Luca

Visconti, Paolo

Visentin, Chiara

Visentin, Francesco

Visescu, Mircea

Vishe, Mahesh

Vishnyakov, Vladimir

Visini, Francesco

Visioli, Antonio

Viskovic, Alberto

Visković, Ivana

Višniakov, Nikolaj

Višnjevec, Ana Miklavčič

Višnjić, Aleksandar

Viso, Alma

Visuri, Ville-Valtteri

Viswanath, P.V.

Vita, Giulio

Vita, Vasiliki

Vitale, Enza 
Vitale, Gianpaolo

Vitale, Luca

Vitale, Marilena

Vitale, Marina Consuelo

Vitali, Andrea

Vitanza, Alessandra

Vitázek, Ivan

Vithanage, Randika

Vitiello, Elisa

Vitillo, Jenny G.

Vítová, Milada

Vittadello, Michele

Viveiros, Carla

Vivoda Prodan, Martina

Vizard, David

Vizovišek, Matej

Vizza, Pasquale

Vizza, Patrizia

Vlachokostas, Christos

Vlachos, Nicholas

Vlachostergiou, Aggeliki

Vlădăreanu, Victor

Vlădeanu, Călin

Vladimir, Kodnyanko

Vladutescu, Stefan

Vladyko, Andrei

Vlahakis, Eleftherios

Vlainić, Josipa

Vlaj, Damjan

Vlantis, Panagiotis

Vlasak, Miloslav

Vlaskin, Mikhail

Vlčková, Miroslava

Vo, Duc-Thang

Vochin, Marius

Voepel, Pascal

Vogel, Thomas

Vogiatzis, Chrysafis

Vogiatzis, Dimitrios

Vogt, Dominik Walter

Vogt, Kristiina

Vohník, Martin

Vohra, Yogesh K.

Voicu, Rodica

Voiculescu, Irina

Voinea, Lelia

Voinescu, Alexandra

Voit, Klaus

Vojisavljevic, Vuk

Vojtek, Martin

Vojtisek-Lom, Michal
Vojtova, Lucy

Volaire, Florence

Volcho, Konstantin

Volek, Tomáš

Voliani, Valerio

Volk, Wolfram

Volkau, Ihar

Volkov, Alexey

Volkova, Elena G.

Volkova, Natalia

Volkwein, Axel

Vollbrecht, Claudia

Volná, Eva

Volny, Michael

Voloboy, Alexey G.

Volochaev, Vadim

Volodin, Alexander M.

Volodymyr, Tkachenko

Vologzhanina, Anna

Volosencu, Constantin

Voloshin, Arkady

Volpe, Annalisa

Volpe, Giovanni

Volpe, Rosaria

Volpp, Joerg

Von Bloh, Werner

Von Langermann, Jan

Von Neuhoff, Nils

Von Tüllenburg, Ferdinand

Von Tycowicz, Christoph

Vona, Marco

Vonásek, Vojtěch

Vončina, Danjel

Vonitsanos, Gerasimos

Vora, Hitesh

Vordos, Nick

Vorob'ev, Mikhail M.

Vorobieff, Peter V.

Voronov, Andriy

Vorontsov, Alexander

Voroshilova, Anzhelika

Vorraro, Giovanni

Vos, Francois Cornelius

Vos, Hendrik

Vosinakis, Spyros

Vosoughifar, Hamidreza

Vostrikova, Kira E.

Vougioukas, Emmanouil

Vourdoubas, John

Vourlias, George

Vouros, Ioannis 
Vourvopoulos, Athanasios

Voutetaki, Maristella E.

Vovides, Yianna

Vozárová, Anna

Voznesenskii, Aleksandr S.

Voznyak, Yuri

Vozzi, Giovanni

Vrána, Radek

Vranceanu, Diana M.

Vrančić, Damir

Vrancken, Bey

Vražić, Mario

Vrbanac, Zoran

Vrbka, Jaromír

Vrettos, Christos

Vriesekoop, Frank

Vrsaljko, Domagoj

Vrscaj, Borut

Vrysis, Lazaros

$\mathrm{Vu}$, Mai The

$\mathrm{Vu}$, Tuan

VU, Viet-Hung

Vuchkov, Todor

Vucinic, Dean

Vuillaume, Jean-Francois

Vujaklija, Ivan

Vujević, Slavko

Vujović, Igor

Vukoje, Marina

Vukomanovič, Mariija

Vukovic, Marin

Vukovic, Natasha

Vulcano, Alfonso

Vuletic, Dijana

Vulic, Milivoj

Vulin, Domagoj

Vuolio, Tero

Vuorinen, Jukka

Vuts, Jozsef

Vuye, Cedric

Vyas, Agin

Vyas, Shruti

Vyhnánek, Tomáš

Vyrlas, Panagiotis

Wa Li, Danny Hin

Wabnitz, Stefan

Wacławek, Stanisław

Wada, Chikamune

Wadati, Hiroki

Wade, Matthew

Waegeman, Willem
Wagenknecht, Udo

Wagner, Herbert

Wagner, Stefan

Wahaia, Faustino

Wahde, Mattias

Wahiduzzaman, Md

Wahlström, Mikael

Wahsheh, Heider A. M.

Waindok, Andrzej

Wajima, Takaaki

Wakjera, Eshetu Janka

Wal, R. L. Vander

Wałach, Daniel

Walas, Krzysztof

Walcek, Chris

Walczak, Katarzyna

Walczak, Krzysztof

Walczak, Paweł

Walczak, Renata

Walda, Jan

Waldhelm, Andy

Waldmann, Danièle

Walendziuk, Wojciech

Walensky, Justin

Walker, A Duncan

Walker, Chandler L.

Walker, David

Walker, Jacqueline

Walker, Patrick

Walkowiak, Krzysztof

Wall, Friederike

Wall, Johan

Wallace, David R.

Wallace, Heather M.

Wallace, Manolis

Walley, John

Walsh, James

Walter, Charles

Walter, Michael S. J.

Waltl, Michael

Walubita, Lubinda

Wan, Hong

Wan, Shibiao

Wan, Tao

Wandosell, Gonzalo

Wandowski, Tomasz

Wang, A-Cheng

Wang, Aining

Wang, Bing

Wang, Bo (Germany)

Wang, Bo (USA) 


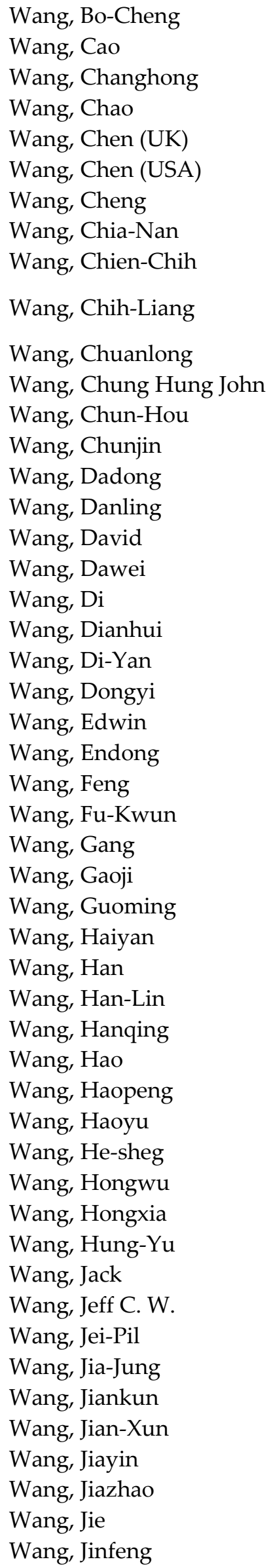

Wang, Jing

Wang, Jingbo

Wang, Jing-Doo

Wang, Jingyi

Wang, Joanna

Wang, Ju

Wang, Julian

Wang, Junjie

Wang, Junye

Wang, Kai (Children's Hospital

Bostondisabled)

Wang, Kai (Texas A\&M University)

Wang, Kainan

Wang, Kui

Wang, Kun Ching

Wang, Kunching

Wang, Kuo-Lung

Wang, Kuo-Ying

Wang, Lefan

Wang, Lei

Wang, Li

Wang, Lidai

Wang, Ling

Wang, Liu

Wang, Longhai

Wang, Meng

Wang, Mian

Wang, Mian C.

Wang, Miao Ling

Wang, Michael Cai

Wang, Min

Wang, Mingkang

Wang, Pei

Wang, Pei Jen

Wang, Peng (UK)

Wang, Peng (USA)

Wang, Pi-Chung

Wang, Ping

Wang, Qian (Singapore)

Wang, Qian (USA)

Wang, Qiancheng

Wang, Qing

Wang, Qingsong

Wang, Qinyi

Wang, Renjie

Wang, Shanshan

Wang, Shaobin

Wang, Shaohui

Wang, Sheng

Wang, Shidan

Wang, Shiow-Luan 
Wang, Shu

Wang, Shuai

Wang, Shun

Wang, Shuo

Wang, Shuohong

Wang, Shuolun

Wang, Sishuo

Wang, Songlin

Wang, Tao

Wang, Tianhao

Wang, Weijie

Wang, Weizhuo

Wang, Wen-Cheng Vincent

Wang, Wenguan

Wang, Wenshuo

Wang, Wensong

Wang, Wentian

Wang, Xiang

Wang, Xiangxue

Wang, Xianzhi

Wang, Xiaodong

Wang, Xiaofeng

Wang, Xiaolin

Wang, Xiaoming

Wang, Xizu

Wang, $\mathrm{Xu}$

Wang, Xuan

Wang, Xuefeng

Wang, Xuemin

Wang, Xuewei

Wang, Ya-huei

Wang, Yaw-Juen

Wang, Yi

Wang, Yifei

Wang, Yi-Hsien

Wang, Yi-Hui

Wang, Yingying

Wang, Yinzhi

Wang, Yonggang

Wang, Yongqiang

Wang, Yongxiang

Wang, Youjin

Wang, Yu (Singapore)

Wang, Yu (USA)

Wang, Yuan

Wang, Yu-Cheng

Wang, Yuejian

Wang, Zejiang

Wang, Zhan

Wang, Zhaoyang

Wang, Zheng
Wang, Zhenpei

Wang, Zhiguang

Wang, Zhiliang

Wang, Zhongyang

Wang, Ziran

Wani, Owies M.

Wan-Wendner, Lin

Wan-Wendner, Roman

Warczek, Jan

Ward, Christopher

Wardal, Witold Jan

Wardeh, George

Warfel, Jaycob D.

Warguła, Łukasz

Warisawa, Shin'ichi

Warnat, Stephan

Warner, Daniel

Warnick, Karl F.

Warren, David

Warwell, Marcus V.

Wascko, Morgan

Wase, Nishikant

Waseem, Owais

Washio, Ayako

Washizawa, Yoshikazu

Wasik, Michał

Wasilczuk, Michal

Wasilewska-Błaszczyk, Monika

Wasilewski, Marek

Wasilkowski, Daniel

Wasowicz, Marcin

Wąsowski, Jacek

Wasserman, Rina

Wasterlain, Sofia

Wasternack, Claus

Waszczykowski, Michał

Watanabe, Haruna

Watanabe, Hiromichi

Watanabe, Kazunori

Watanabe, Masanori

Watanabe, Wataru

Watanabe, Yasumasa

Watarai, Hitoshi

Waterlot, Christophe

Watier, Bruno

Watorek, Marcin

Watson, Alan

Watson, Des

Watson, Lisa

Watson, Neville

Watson, Richard 
Watson, Roger

Watson, Simon

Watter, Holger

Watts, Greg

Wautelet, Yves

Waxman, Michael

Wayu, Mulugeta

Ważny, Mariusz

Wcislik, Miroslaw

Wcislo, Grzegorz

Wdowin, Magdalena

Weaver, Paul

Weaver, Wayne W.

Webb, Ann

Webb, R. Clinton

Weber, Achim

Weber, Cornelius

Weber, Eric S.

Weber, Felix

Weber, Griffin M.

Weber, Robert

Weber, Roland

Weems, Andrew C.

Wegiel, Tomasz

Węglarski, Mariusz

Weglarz, Wladyslaw

Węglorz, Marek

Wegrzyn, Pawel

Węgrzyńska, Małgorzata

Węgrzyński, Wojciech

Węgrzyn-Wolska, Katarzyna

Wehner, Michael

Wehrle, Erich

Wei, Chengli

Wei, Da-Hua

Wei, Daixiu

Wei, Debing

Wei, Jianqiang

Wei, Meng

Wei, Qi-Huo

Wei, Wei

Wei, Xialu

Wei, Xiupeng

Wei, Xuebin

Wei, Yang

Wei, Yuyang

Wei, Zhongbao

Wei, Zhu

Weidinger, Tamás

Weidner, Robert

Weigand, Wolfgang
Weigelt, Matthias

Weiler, Stephan

Weinberger, Markus

Weingaertner, Tim

Weis, Judith S.

Weiss, Avi

Weiss, David

Weiss, Karl-anders

Weiss, Zdenek

Weiss, Zeev

Weiszer, Michal

Weitschek, Emanuel

Weitzen, Jay A.

Weizman, Yehuda

Welch, Tré Raymond

Weldehawaryat, Goitom Kahsay

Welle Donker, Frederika

Welle, Alexander

Weller, Derek J.

Wells, Tracy

Wen, Changyun

Wen, Chih-Yu

Wen, Chunsheng

Wen, Chunyi

Wen, Han

Wen, Huei-Jhen

Wen, Jianming

Wen, Li

Weng, Rui-Chian

Weng, Yung-Jin

Wen-Jeng, Ho

Wenk, Hans-Rudolf

Wenzel, Hans

Wenzel, Tobias

Werbińska-Wojciechowska, Sylwia

Werder, Julia Von

Weres, Jerzy

Werman, Michael

Werner, Kurt

Werner, Małgorzata

Werner, Nachbauer

Wertz, Philip W.

Wesołowski, Marcin

Wessel, Dulcineia F.

West, Andrew

West, Shaun

Westermann, Rüdiger

Westhoff, Mike-Andrew

Weyn, Maarten

Whang, Mincheol

Wheeler, Thomas 
Whidborne, James

Whillier, Stephney

Whisler, Daniel

White, Craig

White, Greg

White, Josh S.

White, Martin

White, Peter

White, Ralph E.

White, Ryan M.

Whiteley, Hugh

Whitney, Erin

Wiącek, Joanna

Wiart, Joe

Wibowo, Hartanto

Wibowo, Santoso

Wichliński, Michał

Wick, Thomas

Wiczyński, Grzegorz

Widbiller, Matthias

Widenmeyer, Marc

Widera, Barbara

Widerska-Chadaj, Zaneta S

Widomski, Marcin K.

Widyaya, Vania Tanda

Widziewicz-Rzońca, Kamila

Wieczorek, Andrzej

Wieczorek, Aneta

Wieczorek, Kinga

Wieczorek, Łukasz

Wieczorek, Rafal M.

Wieczorowski, Michal

Wielgosiński, Grzegorz

Wieringa, Fokko

Wiernik, Brenton M.

Wierzbicka, Anna

Wierzbicka, Elżbieta

Wierzbicki, Jędrzej

Wierzbicki, Sławomir

Wiesner, Stefan

Wietfeldt, Fred

Wijesinghe, Ruchire Eranga

Wiktorski, Tomasz

Wilczek, Brigitte

Wilczyński, Jacek

Wild, Jochen

Wildani, Avani

Wildemann, Britt

Wiley, Robert

Wilhelm, Johannes

Wilhelm, Katrin
Wilhelm, Kerstin

Wilk, Andrzej

Wilk, Izabela

Wilke, Andre

Wilkowska, Agnieszka

Wilkowski, Artur

Willberg, Christian

Willems, Mark Elisabeth Theodorus

Willett, Peter

Williams, Crispin

Williams, Gabriel J.

Williams, Jane

Williams, Jeremiah

Williams, John A.

Williams, Kathryn R.

Williams, Roger

Williams, Stephen

Willis, Anusuya

Willmott, Sandy

Wilson, Adrian

Wilson, Anne M.

Wilson, Claudia

Wilt, Michael

Wimbush, Stuart

Wimmer, Reinhard

Win, Thomas

Winczek, Jerzy

Windholz, Laurentius

Winiarski, Tomasz

Winkler, Anja

Winocur, Efraim

Winocur, Ephraim

Winterfeld, Andreea

Winters, Brandon J.

Winther, Ole

Wiora, Alicja

Wiseman, Yair

Wisner, Brian J.

Wisniewski, Adam

Wiśniewski, Piotr

Wiśniewski, Remigiusz

Wiśniewski, Tomasz

Wiśniowska, Ewa

Witaszek, Kamil

Witczak, Marcin

Witczak, Pawel

Witczak, Piotr

Witczak, Stanislaw

Witham, Claire

Witjes, Max J.H.

Witkowska Nery, Emilia 
Witkowski, Jarosław

Witkowski, Wojciech

Witos, Miroslaw

Witte, Hartmut

Wittemann, Alexander

Witt-Enderby, Paula

Wittich, Christine E.

Wittmann, Folker H.

Wittmann, Jürgen

Witzel, Bernd

Wiwatanapataphee, Benchawan

Włoch, Marcin

Włodarczyk, Maciej

Włodarczyk, Patryk

Włodarczyk, Paweł P.

Włodarczyk-makuła, Maria

Włodarczyk-Stasiak, Marzena

Wnorowski, Andrzej

Wnuk, Krzysztof

Woche, Susanne K.

Woda, Marek

Wódkiewicz, Maciej

Wohlrab, Sebastian

Wöhrer, Alexander

Wójciak, Karolina

Wojciech, Giernacki

Wojciech, Gilewski

Wojciechowicz-Budzisz, Agata

Wojciechowski, Jerzy

Wojciechowski, Krzysztof Witold

Wojciechowski, Łukasz

Wojciechowski, Szymon

Wojciechowski, Tomasz

Wojcieszak, Robert

Wójcik, Artur

Wójcik, Dariusz

Wojcik, Grzegorz

Wójcik, Grzegorz Marcin

Wójcik, Janusz

Wójcik, Krzysztof

Wojewoda, Jerzy

Wojkiewicz, Jean-Luc

Wojnicki, Igor

Wojnicki, Marek

Wojnicz, Wiktoria

Wojs, Marcin K.

Wojszczyk, Rafał

Wojtowicz, Konrad

Wójtowicz, Ryszard

Wojtunik-Kulesza, Karolina Anna

Wojtusik, Mateusz
Wojtyczka, Robert

Wojtyla, Łukasz

Wojtysiak, Dorota

Wolański, Piotr

Wolanśki, Wojciech

Wolarz, Eryk

Woldemariam, Daniel

Wolfe, Tonya B.

Wolfel, Richard L.

Wolfrum, Edward J.

Wolkenberg, Paulina

Wollesen, Bettina

Wollweber, Juergen

Wolniak, Radosław

Wolny, Ada

Wołosz, Krzysztof J.

Woloszyk, Anna

Wolszczak, Piotr

Wolterink, Jelmer

Won, Daehan

Won, Jeong-Hun

Won, Myounggyu

Won, Myoung-Soo

Won, Yoo-Seung

WONG, Florence Mei Fung

Wong, Harris

Wong, Jenna

Wong, Kin-hong

Wong, Ling Tim

Wong, M. L.

WONG, Marianne P. Y.

Wong, Raymond

Wong, Tzu-Tsung

Wong, Voon-Loong

Wong, Wai

Wong, Yujun

Wong, Zijing

Wongkasem, Nantakan

Wongsaroj, Wongsakorn

Wonhyuk, Cho

Woo, Dong Sik

Woo, Hanwool

Woo, Ji Young

Woo, Seong Tak

Woo, Wai Lok

Wood, Antony

Wood, David

Wood, Scott T.

Wood, Stephen L.

Woodfield, Peter

Woodford, Brendon 
Woodhouse, Jim

Woodward, David

Workamp, Marcel

Workie, Bizuneh

Worsey, Matthew To

Worthen, Andrew J.

Wortmann, Martin

Wos, Piotr

Wosatko, Adam

Woschke, Elmar

Wöstemeyer, Johannes

Wöstmann, Bernd

Wotawa, Franz

Wotruba, Hermann

Wotzka, Daria

Woubishet, Taffese

Wouter, Botte

Wouters, Michael J.

Wouters, Peter

Woźniak, Mateusz

Wrenger, Burkhard

Wright, Andrew John

Wright, Catherine

Wróbel, Karol

Wróbel, Krzysztof

Wróbel, Marek

Wrobel, Michal R.

Wrobel, Piotr

Wrobel, Rafał

Wróblewski, Roman

Wrona, Paweł

Wronkowicz-Katunin, Angelika

Wronski, Torsten

Wrzesińska, Magdalena

Wrzesiński, Grzegorz

$\mathrm{Wu}$, Ben

$\mathrm{Wu}$, Chensheng

$\mathrm{Wu}, \mathrm{Chia-Wen}$

$\mathrm{Wu}$, Chienhsun

Wu, Chun Ho

Wu, Chyan-Chyi

$\mathrm{Wu}$, Dayong

$\mathrm{Wu}, \mathrm{Di}$

$\mathrm{Wu}$, Fan

Wu, Fang-Tzy

$\mathrm{Wu}$, Guoyuan

$\mathrm{Wu}$, Haimeng

$\mathrm{Wu}$, Huaming

$\mathrm{Wu}$, Hung-Chin

Wu, Hwai-Chung

Wu, Jiang
$\mathrm{Wu}$, Jian-Hong

$\mathrm{Wu}$, Jong-Wuu

$\mathrm{Wu}$, Jyh-Horng

$\mathrm{Wu}, \mathrm{Kai}$

WU, Lihua

$\mathrm{Wu}$, Lili

$\mathrm{Wu}$, Mingtao

$\mathrm{Wu}$, Molei

$\mathrm{Wu}$, Nan-Jing

Wu, Peng

$\mathrm{Wu}$, Ruey-Beei

$\mathrm{Wu}$, Shaoju

$\mathrm{Wu}$, Shenghua

Wu, Shiann Ming

$\mathrm{Wu}$, Shih-Jeh

$\mathrm{Wu}$, Shin-Tson

$\mathrm{Wu}$, Shuoxing

$\mathrm{Wu}$, Suiwen

$\mathrm{Wu}$, Xiaofeng

Wu, Xin-Ping

$\mathrm{Wu}$, Xuejian

$\mathrm{Wu}$, Yan (Singapore)

$\mathrm{Wu}$, Yan (USA)

$\mathrm{Wu}$, Yaqiao

$\mathrm{Wu}, \mathrm{Yi}$

$\mathrm{Wu}, \mathrm{You}$

$\mathrm{Wu}$, Yuan-Kang

$\mathrm{Wu}$, Yuanwei

$\mathrm{Wu}, \mathrm{Yu}$-Ting

$\mathrm{Wu}, \mathrm{Zan}$

Wulandana, Rachmadian

Wünsche, Christine

Wurz, Marc Christopher

Wuttke, Heinz-Dietrich

Wuttke, Manfred

Wydra, Michal

Wyffels, Francis

Wynn, Peter

Wyrick, Joshua R.

Wysocki, Marian

Wysokinski, Karol Izydor

Wystalska, Katarzyna

Wyszkowska, Patrycja

Wyszyński, Dominik

Wzorek, Małgorzata

Xavier, José Manuel Cardoso

Xavier, Neyt

Xavier, Raquel

Xenakis, Apostolis

Xenidis, Yiannis

Xhafa, Fatos 


\begin{tabular}{|c|c|}
\hline Xi, Xiaodan & Xu, Chao \\
\hline Xia, Changlei & Xu, Chaoshui \\
\hline Xia, Fei & $\mathrm{Xu}$, Fei \\
\hline Xia, Feihong & $\mathrm{Xu}, \mathrm{Gu}$ \\
\hline Xia, Kelin & Xu, Jian (Japan) \\
\hline Xia, Menghan & Xu, Jian (USA) \\
\hline Xia, Ting & $\mathrm{Xu}$, Jiaqi \\
\hline Xiang, Chaoqun & Xu, Jun \\
\hline Xiang, Gong & Xu, Jun-Li \\
\hline Xiang, Junting & Xu, Kai \\
\hline Xiang, Ning & $\mathrm{Xu}$, Lang \\
\hline Xiang, Sheng & Xu, Pengfei \\
\hline Xiang, Yujiang & Xu, Rongguang \\
\hline Xiao, Bo & Xu, Sutie \\
\hline Xiao, Heng & Xu, Tianhua \\
\hline Xiao, Liangliang & $\mathrm{Xu}$, Tingbao \\
\hline Xiao, Perry & Xu, Xiaodong \\
\hline Xiao, Rui & Xu, Xiaojie \\
\hline Xiao, Shaoping & Xu, Xiaoyin \\
\hline Xiao, Sun & Xu, Xinhao \\
\hline Xiao, Xin & Xu, Xuesong \\
\hline Xiao, Xuesu & Xu, Yan \\
\hline Xiao, Ye & $\mathrm{Xu}, \mathrm{Yi}$ \\
\hline Xiao, Yegui & $\mathrm{Xu}$, Yong \\
\hline Xiao, Yong & Xu, Yuandong \\
\hline Xiao, Zhongmin & $\mathrm{Xu}$, Yuquan \\
\hline Xie, Haibo & Xu, Yusheng \\
\hline Xie, Haiyan & Xue, Hai \\
\hline Xie, Jiafeng & Xue, Yifei \\
\hline Xie, Rui & Yaakoubi, Nourdin \\
\hline Xie, Shangran & Yabuki, Soichi \\
\hline Xie, Shengkun & Yabuuchi, Yoshiyuki \\
\hline Xie, Tianyu & Yadav, Mamta \\
\hline Xie, Ting & Yadav, Sakshi \\
\hline Xie, Xiang & Yadav, Sushma \\
\hline Xie, Xinfeng & Yadav, Vinay \\
\hline Xie, Xiufeng & Yagami, Kimitoshi \\
\hline Xie, Yuliang & Yagasaki, Takuma \\
\hline Xie, Yun & Yager, Zali \\
\hline Xie, Zhijian & Yagihara, Shin \\
\hline Xie, Zhong-Ru & Yaguchi, Hiroyuki \\
\hline Ximenes, Arimatéa C. & Yaguchi, Yuichi \\
\hline Xin, Guiyang & Yahiaoui, Reda \\
\hline Xing (Yan), Yangang & Yakaboylu, Gunes A. \\
\hline Xing, Jin & Yakirevich, Alexander \\
\hline Xing, Yuxin & Yakout, Mostafa \\
\hline Xiong, Lin & Yakovlev, Vladislav \\
\hline Xiong, Siting & Yakunin, Alexander \\
\hline Xiong, Zehui & Yakush, Sergey E. \\
\hline Xofis, Panteleimon & Yalavarthi, Rambabu \\
\hline $\mathrm{Xu}, \mathrm{Bin}$ & Yalcinkaya, Fatma \\
\hline
\end{tabular}


Yamabuki, Kazuhiro

Yamada, Satoru

Yamada, Taketo

Yamada, Yukio

Yamagishi, Kento

Yamagishi, Masao

Yamagiwa, Shinichi

Yamaguchi, Hiroshi

Yamaguchi, Satoshi

Yamaguchi, Tomoyuki

Yamakawa, Hiroyuki

Yamamoto, Hajime

Yamamoto, Tatsuyuki

Yamaoka, Yusuke

Yamasaki, Yuichi

Yamashita, Yousuke

Yamauchi, Takashi

Yamazaki, Hirohito

Yamin, Muhammd Mudassar

Yan, Bingxi

Yan, Chao

Yan, Fuyao

Yan, Hui

Yan, Mary

Yan, Xinggang

Yan, $\mathrm{Xu}$

Yan, Yuanwei

Yanagawa, Aya

Yanagisawa, Daichi

Yanagisawa, Naoko

Yanase, Takashi

Yanaseko, Tetsuro

Yanda, Murali

Yaneva, Zvezdelina L.

Yáñez, Dionisio F.

Yang, Bo (Japan)

Yang, Bo (USA)

Yang, Cancan

Yang, Chao-Lung

Yang, Chao-Tung

Yang, Cheng

Yang, Cheng-Fu

Yang, Cheng-Jung

Yang, Chenguang

Yang, Chih-Cheng

Yang, Chih-Chin

Yang, Chih-Te

Yang, Chun-Wei

Yang, Ci Jian

Yang, Di

Yang, Dujuan
Yang, Fajun

Yang, Fan

Yang, Fuqian

Yang, Guang

Yang, Haizhao

Yang, Hee-Deok

Yang, Heng

Yang, Hsiao-Yu

Yang, Jiamiao

Yang, Jiawei

Yang, Jidong

Yang, Jie

Yang, Judy P.

Yang, Kai Min

Yang, Kailun

Yang, Kwangsoo

Yang, Linda

Yang, Mijia

Yang, Qian

Yang, Quanlong

Yang, Ray-Yeng

Yang, Ruixin

Yang, Sai Wei

Yang, Sejung

Yang, Seung-hak

Yang, Shan

Yang, Shen

Yang, Sheng

Yang, Shiyu

Yang, Shujun

Yang, Shun-Fa

Yang, Sungwoo

Yang, Suo

Yang, Tai-Hua

Yang, Tao

Yang, Tiannan

Yang, Wencheng

Yang, Wenxian

Yang, Wenzhi

Yang, Xiaoyun

Yang, Xinbo

Yang, Xiong

Yang, Xusan

Yang, Yang

Yang, Yeongwook

Yang, Yezhou

Yang, Yifan

Yang, Ying

Yang, Yiran (Emma)

Yang, Yongkang

Yang, Yuan-Sen 


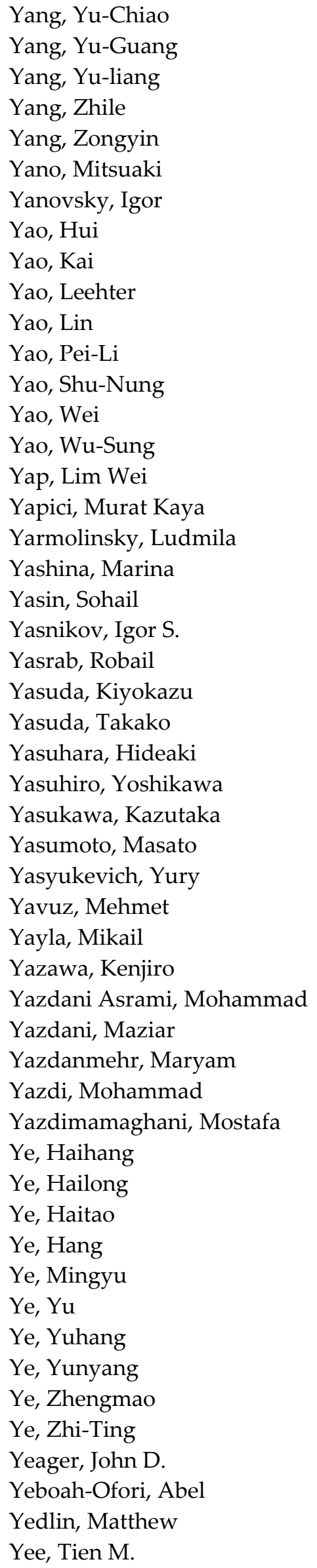

Yeh, Chih-Ko

Yeh, Tsu-Ming

Yeh, Yin-Ting

Yelisetti, Subbarao

Yemelyanov, Vitaliy A.

Yemmireddy, Veerachandra K.

Yen, Benjamin

Yen, Chia-Hung

Yenes, Mariano

Yeo, ChangSeob

Yeo, Joo Chuan

Yeoh, Justin K. W.

Yeom, Chunho

Yeom, Soonja

Yeon, Jaeheum

Yeong, Wai Yee

Yepuri, Nageshwar R.

Yerushalmi, Laleh

Yeum, Chul Min

Yeung, Andy Wai Kan

Yi, Byung-Ju

Yi, Hwang

Yi, June-Seong

$\mathrm{Yi}$, Junsin

Yi, Wen

Yi, Won-Jae

$\mathrm{Yi}, \mathrm{Zao}$

Yi, Zhengkun

Yiannopoulos, Kostas

Yick, Kit-Lun

Yihun, Yimesker

Yildirim Yurusen, Nurseda

Yildirim, Yalcin

Yildiz, Bilge Can

Yilmaz, Mehmet

Yilmaz, Muhittin

Yim, Seongjin

Yin, Chuntao

Yin, Fei

Yin, Feijia

Yin, Peng-Yeng

Yin, Zhen

Yin, Zhouyang

Yiouta-Mitra, Paraskevi

Yitmen, Ibrahim

Yngvesson, Sigfrid

Yo, Tanaka

Yodo, Nita

Yohei, Endo

Yokoi, Fumiaki

Yokota, Tomoyuki 
Yokoyama, Kazushige

Yokoyama, Takashi

Yokoyama, Yasuo

Yoneyama, Satoru

Yoneyama, Tadakatsu

Yonezawa, Tetsu

Yoo, Byungseok

Yoo, Chang Geun

Yoo, Do Guen

Yoo, Dong Jin

Yoo, Hah Young

Yoo, Hosik

Yoo, Jaehyun

Yoo, Jeeyoung

Yoo, Jung Sun

Yoo, Kwan-Hee

Yoo, Mintaek

Yoo, So Young

Yoo, Sung Jin

Yoon, Hyeonseok

Yoon, Hyoseok

Yoon, Hyun Chul

Yoon, Hyungchul (Henry)

Yoon, Hyung-Koo

Yoon, In-Seok

Yoon, Insun

Yoon, jeoung Seok

Yoon, Jonghun

Yoon, Keun-Young

Yoon, Sangpil

Yoon, Tae-Hoon

Yoon, Won-Sup

Yoon, Yeojoon

Yoon, Yong-Ik

Yordanova, Snejana

Yörük, Can Rüstü

Yoshida, Aya

Yoshida, Hiroshi

Yoshida, Katsunori

Yoshida, Sanichiro

Yoshida, Shigeo

Yoshikawa, Hiroki

Yoshikawa, Hiroshi

Yoshimura, Takenori

Yoshizawa, Shingo

Yoshizawa, Shinya

You, Byoung He

You, Cheol-Hwan

You, Greg

You, Ilhwan

You, Jeong Ho
You, Jiing-Yun

You, Lingyun

You, Shutang (Steve)

You, Won-hee

You, Young-Hwan

Youd, Leslie

Youn, Heejung

Youn, Hyun Jo

Youn, Ik-Hyun

Young, Cara E Capitena

Young, Chase

Young, Lori A.

Young, Timothy M.

Younis, Mustafa Z.

Yousaf, Jawad

Yousef, Samy

Yousefian, Sajjad

Yousefianmoghadam, Seyedsina

Youssef, Tarek

Yperman, Jan

Yrälä, Kim

Yu, Bor-Yih

$\mathrm{Yu}$, Chenglong

Yu, Chih-Chang

$\mathrm{Yu}$, Chung-Hsien

$\mathrm{Yu}$, Feng

$\mathrm{Yu}$, Fu-wing

Yu, Haitao

Yu, Ha-Jin

$\mathrm{Yu}, \mathrm{HaO}$

$\mathrm{Yu}$, Hong-Ren

$\mathrm{Yu}$, Huangchao

Yu, Jaesok

$\mathrm{Yu}$, Jiangfan

$\mathrm{Yu}$, Jorn

Yu, Jung-Doung

Yu, Kaiyan

$\mathrm{Yu}$, Liangjiang

$\mathrm{Yu}, \mathrm{Lu}$

Yu, Manzhu

Yu, Miao

Yu, Ning

Yu, Ollie Yiru

Yu, Pao-Shan

Yu, Ping

$\mathrm{Yu}$, Ruei-Sung

$\mathrm{Yu}$, Ruozhou

Yu, Shaode

Yu, Shujian

$\mathrm{Yu}$, Ting-To

Yu, Wei 


\begin{tabular}{|c|c|}
\hline Yu, Wenbin & Zaccone, Claudio \\
\hline Yu, Wen-der & Zaccone, Raphael \\
\hline Yu, Wenwei & Zacharaki, Evangelia I. \\
\hline Yu, Xiaoming & Zacharatos, Filimon \\
\hline Yu, Xinting & Zacharewicz, Gregory \\
\hline Yu, Yanbo & Zachariáš, Jiří \\
\hline Yu, Yang & Zachiu, Cornel \\
\hline Yu, Ziwen & Zadobrischi, Eduard \\
\hline Yuan, Guanghui & Zadra, Claudia \\
\hline Yuan, Hao & Zadrozny, Wlodek \\
\hline Yuan, Jie & Zaffora, Andrea \\
\hline Yuan, Qingcong & Zafra, Amelia \\
\hline Yuan, Shyan-Ming & Zagajewski, Bogdan \\
\hline Yuan, Tao & Zagalaz-Anula, Noelia \\
\hline Yuan, Tianfeng & Zagan, Ionel \\
\hline Yuan, Weijie & Žagar, Dejan \\
\hline Yuan, Wenan & Zaghari, Bahareh \\
\hline Yuan, Wenzhen & Zaghloul, Mohamed \\
\hline Yuan, Xiao-hui & Zagorskas, Jurgis \\
\hline Yuan, Xue-Ming. & Zagrodny, Bartłomiej \\
\hline Yudin, Dmitry & Zagubień, Adam \\
\hline Yue, Shengying & Zaguła, Grzegorz \\
\hline Yuen, Anthony Chun Yin & Zäh, Michael \\
\hline Yuen, Chau & Zaharescu, Traian \\
\hline Yuen, Peter & Zaharia, Carmen \\
\hline Yuko, Nishimoto & Zaharia, Mihai \\
\hline Yun, Byoung-Ju & Zaharis, Zaharias \\
\hline Yun, Il Dong & Zahínos, Emilio Viñuelas \\
\hline Yun, Ji-Hoon & Zahoranova, Anna \\
\hline Yung, Hong-Wa & Zahorian, Stephen A. \\
\hline Yung, Tung-Yuan & Zaidan, Martha Arbayani \\
\hline Yunusa-Kaltungo, Akilu & Zaidi, Ali \\
\hline Yurchenko, Olga & Zaimes, George \\
\hline Yurkevich, Nataliya V. & Zaina, Giusi \\
\hline Yurkov, Andrey & Zaitsev, Andrey \\
\hline Yurtsever, Ekim & Zaǐtsev, Sergei Yu \\
\hline Yustres, Ángel & Zaitsev, Vasilii \\
\hline Yuya, Sakai & Zaitseva, Elena \\
\hline Z. Psarakis, Emmanouil & Zajac, Marzena \\
\hline Z. Sampaio, Alcínia & Zajączkowski, Bartosz \\
\hline Zaba, Krzysztof & Zajkowski, Maciej \\
\hline Zabaloy, Santiago & Zak, Andrzej \\
\hline Zabierowski, Wojciech & Żak, Jolanta \\
\hline Zabini, Flavio & Żak, Krzysztof \\
\hline Zabłocka-Godlewska, Ewa & Zakharov, Oleg V. \\
\hline Zaborowicz, Maciej & Zakinyan, Robert \\
\hline Zabulis, Xenophon & Zakłos-Szyda, Malgorzata \\
\hline Zacarías, Francisco Fernández & Zalar, Vesna \\
\hline Zaccagnino, Rocco & Zalewski, Paweł \\
\hline Zacchei, Enrico & Žalga, Artūras \\
\hline Zaccone, Alessio & Zalidis, George \\
\hline
\end{tabular}


Žalimienè, Laura

Zalkow, Frank

Zam, Azhar

Zama, Fabiana

Zamani Meymian, Nima

Zambelli, Cristian

Zambito, Ylenia

Zambon, Alfonso

Zamboni, Giorgio

Zambrano Mendoza, Maria Clemencia

Zambrano, Miller

Zambrano-Martinez, Jorge Luis

Zamfir, Ana-Maria

Zamiri, Ali

Zammit Mangion, Marion

Zamora Mestre, Joan-Lluis

Zamora-Polo, Francisco

Zamparini, Fausto

Zampetti, Emiliano

Zampieri, Marcos

Zampieri, Nicolò

Zampieri, Paolo

Zampogna, Alessandro

Zamuda, Ales

Zamuz, Sol

Zamyatnin, Andrey

Zamzmi, Ghada

Zanetti, Elisabetta

Zang, Liqing

Zang, Xiaoqin

Zangeneh-Nejad, Farzad

Zanin, Massimiliano

Zanini, Mariano

Zannoni, Daniele

Zanotti Fragonara, Luca

Zanuttini, Roberto

Zanuy, Carlos

Zanzotto, Fabio Massimo

Zapała-Sławeta, Justyna

Zapico, Pablo

Zaplana, Isiah

Zappa, Giovanni

Zappa, Massimiliano

Zappalà, Giuseppe

Zappia, Giovanni

Zappia, Stefania

Zarabadi-Poor, Pezhman

Zarabska-Bożejewicz, Daria

Zaras, Nikolaos

Zarbaf, Ehsan

Zarca, Gabriel
Zardin, Barbara

Zare Naghadehi, Masoud

Zare, Mohsen

Zareinia, Kourosh

Zarembski, Allan

Zarepoor, Masoud

Zargar, Omid

Zarnegar, Armita

Zarra, Tiziano

Zarrin, Javad

Zarrintaj, Payam

Zarzuelo, Carmen

Zastempowski, Marcin

Zastrow, Melissa L.

Zatelli, Paolo

Zavašnik, Janez

Zawadzki, Jarosław

Zawadzki, Piotr

Zawadzki, Przemysław

Zawała, Jan

Zawieja, Iwona

Zayets, Vadym

Zbiciak, Artur

Żbikowska, Anna

Zbořil, František

Zdanowski, Maciej

Zdarta, Jakub

Zdenek, Slouka

Ždímalová, Mária

Zdunek, Rafal

Zdyb, Agata

Zdziebko, Tomasz

Zębala, Wojciech

Zebrev, Gennady

Zednik, Ricardo

Zefferer, Thomas

Zehnder, Matthias

Zehtabian, Amin

Zeid, Omar

Zeidi, Mahdi

Zeinali, Yasha

Zelger, Bernhard

Zelinka, Samuel L.

Zeljkovic, Milan

Zema, Demetrio Antonio

Zema, Nicola Roberto

Zeman, Krystof

Zemanová, Alena

Zembaty, Zbigniew

Zemcik, Pavel

Zemková, Erika 
Zemouri, Ryad

Zemtsovskiy, A. V.

Zendo, Takeshi

Zendri, Elisabetta

Zenebe Degefa, Merkebu

Zeng, Hao

Zeng, JI

Zeng, Jia

Zeng, Kai

Zeng, Minxiang

Zeng, Shuwen

Zeng, Xiangyan

Zeng, Yijian

Zeng, Yong

Zenk, Lukas

Zenzeri, Jacopo

Zerbino, Pierluigi

Żesławska, Ewa

Zettsu, Nobuyuki

Zewail, Rami

Zgură, Irina

Zhai, Yao

Zhan, Fangqiong

Zhan, Siyuan

Zhang, Anpeng

Zhang, Binsheng

Zhang, Bowu

Zhang, Chao

Zhang, Chen

Zhang, Chunwei

Zhang, Dan

Zhang, Dayi

Zhang, Derun

Zhang, Dongshi

Zhang, Ershuai

Zhang, Fan

Zhang, Feng

Zhang, Guannan

Zhang, Guiming

Zhang, Han (Texas A\&M University)

Zhang, Han (University of North Carolina)

Zhang, Hangfeng

Zhang, Haolin

Zhang, Haopeng

Zhang, Haoran

Zhang, Hong

Zhang, Hui

Zhang, Ivan

Zhang, Jiachen

Zhang, Jiashu
Zhang, Jiawei

Zhang, Jie

Zhang, Jihui

Zhang, Jize

Zhang, Kaiwen

Zhang, Lei

Zhang, Leo

Zhang, Ligang

Zhang, Lihai

Zhang, Lijie Grace

Zhang, Linjun

Zhang, Lu

Zhang, Meirong

Zhang, Ming

Zhang, Mingshao

Zhang, $\mathrm{Na}$

Zhang, Peng (New York University Tandon

School of Engineering)

Zhang, Peng (Stony Brook University)

Zhang, Peng (The Rockefeller University)

Zhang, Qi

Zhang, Qian (Florida State University)

Zhang, Qian (Johns Hopkins University)

Zhang, Qiangzhe

Zhang, Qichun

Zhang, Qifeng

Zhang, Renyun

Zhang, Ruiqi

Zhang, Ruiyong

Zhang, Runzhou

Zhang, Shanglong

Zhang, Shaolin

Zhang, Shenli

Zhang, Shiming

Zhang, Shiqiang

Zhang, Shu

Zhang, Shuai

Zhang, Shuaifang

Zhang, Su

Zhang, Sung-Uk

Zhang, Tiansheng

Zhang, Tieling

Zhang, Tingting

Zhang, Wei

Zhang, Wen

Zhang, Wenhan

Zhang, Wenjun

Zhang, Wen-Ran

Zhang, Xiang

Zhang, Xianghui

Zhang, Xian-Ming 


\begin{tabular}{|c|c|}
\hline Zhang, Xiaoming & Zhao, Haitao \\
\hline Zhang, Xiaoxin & Zhao, Haiyan \\
\hline Zhang, Xiaoyan & Zhao, Hang \\
\hline Zhang, Xiaoyu & Zhao, Hongyi \\
\hline ZHANG, XINCHANG & Zhao, Jian \\
\hline Zhang, Xinxing & Zhao, Jingzhou \\
\hline Zhang, Xinxuan & Zhao, Lianfeng \\
\hline Zhang, Xu & Zhao, Mingfei \\
\hline Zhang, Xuechen & Zhao, Mingjun \\
\hline Zhang, Xueqiang Alex & Zhao, Ruaikai \\
\hline Zhang, Xuewei & Zhao, Rui \\
\hline Zhang, Yajie & Zhao, Shan \\
\hline Zhang, Yan & Zhao, Wei \\
\hline Zhang, Yang (Florida State University) & Zhao, Wenchao \\
\hline Zhang, Yang (Iowa State University) & Zhao, Xinxin (Cindy) \\
\hline Zhang, Yang (University of Minnesota) & Zhao, Xiubo \\
\hline Zhang, Yang (Western Carolina University) & Zhao, Yingjun \\
\hline Zhang, Yanping & Zhao, Yongxin \\
\hline Zhang, Yao & Zhao, Yu \\
\hline ZHANG, YI (Singapore) & Zhao, Zengfeng \\
\hline Zhang, Yi (USA) & Zhao, Zhiming \\
\hline Zhang, Yicha & Zhao, Zhiqiang \\
\hline Zhang, Yichi & Zhao, Zuomin \\
\hline Zhang, Yi-Fan & Zhbanov, Alexander \\
\hline Zhang, Yijie & Zheng, Bocong \\
\hline Zhang, Youwen & Zheng, Dongdong \\
\hline Zhang, Yu (Singapore) & Zheng, Fei \\
\hline Zhang, Yu (UK) & Zheng, Jian \\
\hline Zhang, Yu (USA) & Zheng, Jinchuan \\
\hline Zhang, Yuanzhi & Zheng, Jun \\
\hline Zhang, Yudong & Zheng, Kai \\
\hline Zhang, Yue & Zheng, Lu \\
\hline Zhang, Yuming & Zheng, Minzhang \\
\hline Zhang, Yun & Zheng, Qiye \\
\hline Zhang, Yuning & Zheng, Songfeng \\
\hline Zhang, Yunyan & Zheng, Xiaochen \\
\hline Zhang, Yuting & Zheng, Xinting \\
\hline Zhang, Zeyu & Zheng, Yihao \\
\hline Zhang, Zhe (Denmark) & Zheng, Yu-Jun \\
\hline Zhang, Zhe (USA) & Zhigang, Chen \\
\hline Zhang, Zheng & Zhirabok, Alexey \\
\hline Zhang, Zhidong & Zhironkin, Sergey \\
\hline Zhang, Zhijian & Zhmud, Boris \\
\hline Zhang, Zhou & Zhmud, Vadim \\
\hline Zhang, Ziqian & Zholobko, Oksana \\
\hline Zhao, Aobo & Zholudev, Maksim S. \\
\hline Zhao, Chunhong & Zhong, Lexuan \\
\hline Zhao, Chunying & Zhong, Liheng \\
\hline Zhao, Dezun & Zhong, Xiangyu \\
\hline Zhao, Gejian & Zhong, Ying \\
\hline Zhao, Guoying & Zhong, Yucheng \\
\hline
\end{tabular}


Zhou, Ao

Zhou, Binbin

Zhou, Chao

Zhou, Chenming

Zhou, Hong

Zhou, Hongyi

Zhou, Hua

Zhou, Jianhui

Zhou, Jing

Zhou, Lai

Zhou, Lin

Zhou, Mengchu

Zhou, Mi

Zhou, Mingqi

Zhou, Mingxi

Zhou, Qiang

Zhou, Qingyu

Zhou, Ran

Zhou, Renjie

Zhou, Shijie

Zhou, Tunhe

Zhou, Wenbin

Zhou, Xianlian

Zhou, Xiao Dong

Zhou, Xiaoyan

Zhou, Xiaozhou

Zhou, Yang

Zhou, Yanlai

Zhou, You

Zhou, Yuekuan

Zhou, Yunlai

Zhou, Yuqing

Zhou, Zheyu

Zhu, Dandan

Zhu, Dianchen

Zhu, Dibin

Zhu, Dongshan

Zhu, Guohun

Zhu, Guoniu

Zhu, Haibin

Zhu, Hongmei

Zhu, Hongyang

Zhu, Juanjuan

Zhu, Junjie

Zhu, Junjun

Zhu, Junxiang

Zhu, Liangdong

Zhu, Lingxiang

$\mathrm{Zhu}, \mathrm{Ni}$

Zhu, Qiji Jim

Zhu, Qingzi
Zhu, Quan

Zhu, Ruoxin

Zhu, Tianyu

Zhu, Tieyuan

Zhu, Xiangwei

Zhu, Xiaoliang

Zhu, Xiaowei

Zhu, Xuan

Zhu, Yang

Zhu, Yi

Zhu, Yong

Zhu, Yuman

Zhu, Yuyang

Zhu, Zijie

Zhuang, Hanqi

Zhukov, Igor

Zhuo, Yue

Zhurakivska, Khrystyna

$\mathrm{Zi}$, Lin

Zia, Kashif

Zia, Muhammad Yousuf Irfan

Ziada, Hassan

Ziarno, Małgorzata

Zicha, Daniel

Zichella, Lorena

Zidanšek, Aleksander

Ziegler, Matthias

Zielina, Michal

Zielinska, Teresa

Zielinska-Dabkowska, Karolina M.

Zielińska-Raczyńska, Sylwia

Zielinski, Adam

Zieliński, Damian

Zieliński, Jacek

Zielinski, Marcin

Zieliński, Rafał

Zielinski, Slawomir

Ziemba, Paweł

Ziemianski, Leonard

Zienert, Tilo

Zieniuk, Bartłomiej

Zierenberg, Johannes

Zietkiewicz, Joanna

Zietz, Carmen

Zikanov, Oleg

Zikria, Yousaf Bin

Zilic, Zeljko

Žilinskas, Antanas

Zima, Beata

Zima, Krzysztof

Zima, Piotr 
Zimbone, Massimo

Zimmer, Dana

Zimmer, J. Christopher

Zimmer, Sebastian

Zimmer, Thomas

Zimmerman, Tahl

Zimmermann, André

Zimon, Dominik

Zinicovscaia, Inga

Zink, Béla

Zink, Matthias Daniel

Zinno, Raffaele

Zinoveev, Dmitry

Ziółko, Mariusz

Ziolkowski, Patryk

Zitoune, Radouane

Zitricky, Vladislav

Zitti, Gianluca

Zivaljic, Nikolina

Živković, Igor

Zivkovic, Irena

Zizzo, Gaetano

Zlatev, Zlatin

Złotek, Urszula

Zlotnik, Sebastian

Žmak, Irena

Zmarzły, Paweł

Zmeureanu, Radu

Žmuk, Berislav

Znamirowska, Agata

Zohm, Hartmut

Zöldy, Máté

Zolfagharian, Ali

Zolliker, Peter

Zoltan-Istvan, Szabo

Żółtowski, Krzysztof

Zomeni, Zomenia

Zonno, Giacomo

Zontone, Pamela

Zorbas, Dimitrios

Zoric, Bruno

Zoričić, Davor

Zorita-Lamadrid, Angel L.

Zorzi, Mattia

Zotovic Stanisic, Ranko

Zotter, Franz

Zotti, Francesca

Zou, Haiyang

Zou, Liang

Zou, Lilong

Zou, Ting
Zou, Xin

Zou, Zheguang

Zou, Zhongwei

Žouželka, Radek

Zovko Končić, Marijana

Zribi, Mehrez

Zrinjski, Mladen

Zsedrovits, Tamás

Zsiborács, Henrik

Zsolt, Kotroczó

Zsuzsanna, Onet-Marian

Zubac, Damir

Zubo, Rana

Zubrzycki, Jarosław

Zucali, Michele

Zucca, Marco

Zucca, Paolo

Zuccari, Fabrizio

Zucchi, Martina

Zucco, Giovanni

Zuccon, Paolo

Żuchowski, Piotr

Zucker, Gerhard

Zuecco, Giulia

Zufferey, Raphael

Zugaza, José Luis

Zuin, Matteo

Zukeran, Akinori

Zukow, Walery

Zukowski, Witold

Žulec, Mirna

Żulewska, Justyna

Zulli, Daniele

Zuluaga, Maria A.

Zumr, David

Zúñiga, Andrés A.

Zunino, Pietro

Zuo, Yi

Zuorro, Antonio

Zupan, Dejan

Župan, Robert

Zupanič, Franc

Zuppolini, Simona

Zúquete, André

Zur, Jonna

Zurauskiene, Nerija

Zurita Ortega, Félix

Zurita, Grover

Zurqani, Hamdi A.

Žuvela, Petar

Zvánovec, Stanislav 
Žvingila, Donatas

Zweiker, David

Zwickagl, Gertrud

Zwierko, Teresa

Zych, Marcin

Zych, Teresa

Zydroń, Paweł

Zydroń, Tymoteusz
Zygmanowski, Marcin

Żyłka, Łukasz

Żyła, Renata

Zysk, Elżbieta

Zyśk, Janusz

Zywica, Grzegorz

Żywicki, Krzysztof

Żyżelewicz, Dorota 